\title{
Uncertainty Quantification in Ocean State Estimation
}

by

\author{
Alexander G. Kalmikov
}

B.Sc., Physics and Mathematics, The Hebrew University of Jerusalem, 1999

M.Sc., Geophysics and Planetary Sciences, Tel-Aviv University, 2006

S.M., Mechanical Engineering, MIT/WHOI Joint Program, 2010

Submitted in partial fulfillment of the requirements for the degree of

Doctor of Philosophy

in Mechanical and Oceanographic Engineering

at the

MASSACHUSETTS INSTITUTE OF TECHNOLOGY

and

WOODS HOLE OCEANOGRAPHIC INSTITUTION

February 2013

(C) 2013 Alexander Kalmikov. All rights reserved.

The author hereby grants to MIT and WHOI permission to reproduce and to distribute publicly paper and electronic copies of this thesis document in whole or in part in any medium now known or hereafter created.

Signature of Author:

Joint Program in Oceanography/Applied Ocean Science and Engineering Massachusetts Institute of Technology and Woods Hole Oceanographic Institution

January 11, 2013

Certified by:

Patrick Heimbach

Principal Research Scientist, MIT

Thesis Supervisor

Certified by:

Carl Wunsch

Cecil and Ida Green Professor of Physical Oceanography, MIT

Thesis Co-Supervisor

Accepted by:

Henrik Schmidt

Professor of Mechanical and Ocean Engineering, MIT

Chair, MIT/WHOI Joint Committee on Applied Ocean Science and Engineering

Accepted by:

David E. Hardt

Ralph E. and Eloise F. Cross Professor of Mechanical Engineering, MIT

Chairman, Committee on Graduate Students, Department of Mechanical Engineering 


\title{
Uncertainty Quantification in Ocean State Estimation
}

\author{
by
}

\author{
Alexander G. Kalmikov \\ Submitted to the Department of Mechanical Engineering at MIT and the Joint \\ Committee on Applied Ocean Science and Engineering at MIT and WHOI \\ on January 11, 2013 in partial fulfillment of the requirements for the \\ Degree of Doctor of Philosophy in Mechanical and Oceanographic Engineering
}

\section{Abstract}

Quantifying uncertainty and error bounds is a key outstanding challenge in ocean state estimation and climate research. It is particularly difficult due to the large dimensionality of this nonlinear estimation problem and the number of uncertain variables involved. The "Estimating the Circulation and Climate of the Oceans" (ECCO) consortium has developed a scalable system for dynamically consistent estimation of global timeevolving ocean state by optimal combination of ocean general circulation model (GCM) with diverse ocean observations. The estimation system is based on the "adjoint method" solution of an unconstrained least-squares optimization problem formulated with the method of Lagrange multipliers for fitting the dynamical ocean model to observations. The dynamical consistency requirement of ocean state estimation necessitates this approach over sequential data assimilation and reanalysis smoothing techniques. In addition, it is computationally advantageous because calculation and storage of large covariance matrices is not required. However, this is also a drawback of the adjoint method, which lacks a native formalism for error propagation and quantification of assimilated uncertainty. The objective of this dissertation is to resolve that limitation by developing a feasible computational methodology for uncertainty analysis in dynamically consistent state estimation, applicable to the large dimensionality of global ocean models.

Hessian (second derivative-based) methodology is developed for Uncertainty Quantification (UQ) in large-scale ocean state estimation, extending the gradient-based adjoint method to employ the second order geometry information of the model-data misfit function in a high-dimensional control space. Large error covariance matrices are evaluated by inverting the Hessian matrix with the developed scalable matrix-free numerical linear algebra algorithms. Hessian-vector product and Jacobian derivative codes of the MIT general circulation model (MITgcm) are generated by means of algorithmic differentiation (AD). Computational complexity of the Hessian code is reduced by tangent linear differentiation of the adjoint code, which preserves the speedup of adjoint checkpointing schemes in the second derivative calculation. A Lanczos algorithm is applied for extracting the leading rank eigenvectors and eigenvalues of the Hessian matrix. The eigenvectors represent the constrained uncertainty patterns. The inverse eigenvalues are the corresponding uncertainties. The dimensionality of UQ calculations is reduced by eliminating the uncertainty null-space unconstrained by the 
supplied observations. Inverse and forward uncertainty propagation schemes are designed for assimilating observation and control variable uncertainties, and for projecting these uncertainties onto oceanographic target quantities. Two versions of these schemes are developed: one evaluates reduction of prior uncertainties, while another does not require prior assumptions. The analysis of uncertainty propagation in the ocean model is timeresolving. It captures the dynamics of uncertainty evolution and reveals transient and stationary uncertainty regimes.

The system is applied to quantifying uncertainties of Antarctic Circumpolar Current (ACC) transport in a global barotropic configuration of the MITgcm. The model is constrained by synthetic observations of sea surface height and velocities. The control space consists of two-dimensional maps of initial and boundary conditions and model parameters. The size of the Hessian matrix is $O\left(10^{10}\right)$ elements, which would require $O(60 \mathrm{~GB})$ of uncompressed storage. It is demonstrated how the choice of observations and their geographic coverage determines the reduction in uncertainties of the estimated transport. The system also yields information on how well the control fields are constrained by the observations. The effects of controls uncertainty reduction due to decrease of diagonal covariance terms are compared to dynamical coupling of controls through off-diagonal covariance terms. The correlations of controls introduced by observation uncertainty assimilation are found to dominate the reduction of uncertainty of transport. An idealized analytical model of ACC guides a detailed time-resolving understanding of uncertainty dynamics.

Keywords: Adjoint model uncertainty, sensitivity, posterior error reduction, reduced rank Hessian matrix, Automatic Differentiation, ocean state estimation, barotropic model, Drake Passage transport.

Thesis Supervisor: Patrick Heimbach

Title: Principal Research Scientist, MIT

Thesis Co-Supervisor: Carl Wunsch

Title: Cecil and Ida Green Professor of Physical Oceanography, MIT 


\section{Dedicated to the memory of Isaac Fievel Kalmykov}

$$
\text { (1923-2010) }
$$

Officer, Engineer, strict and loving grandfather, whose responsibility, discipline and work ethic guided me through the challenges of this dissertation 


\section{Acknowledgment}

I am sincerely grateful to my advisors, Patrick Heimbach and Carl Wunsch, for their generous support, thoughtful guidance and the intellectually inspiring opportunity to work on this interesting project. I am especially indebted to Patrick Heimbach for the many hours he spent helping me overcome technical difficulties and conceptualize the original contributions of this thesis. I would like to thank my doctoral committee members, Pierre Lermusiaux and Michael Spall, for their helpful advise and the thorough reading of the draft of the thesis. I would like to acknowledge my teachers, Youssef Marzouk and Qiqi Wang, for introducing me to the field of Uncertainty Quantification. Youssef Marzouk's class at the "Large-scale Inverse Problems and Quantification of Uncertainty" (2011) workshop at the Institute for Mathematics and its Applications (IMA, University of Minnesota) inspired one of the key algorithms of this dissertation. I am grateful for the opportunity to learn the fundamentals of GFD and physical oceanography from the pioneers in the field - Joe Pedlosky and John Marshall, whose insightful teaching shaped my understanding of the subject.

I would like to express my sincere appreciation to Leslie Regan, the Graduate Administrator in the department of Mechanical Engineering for all the help and the personal attention throughout my graduate program. I thank the administrators of the Joint Program and the staff of the Academic Programs Office at WHOI, especially Julia Westwater, Ronni Schwartz, Marsha Gomes and Valerie Caron, for their warm welcome and the vital support of my studies.

I thank my classmates, who became dear friends, for mutual encouragement and sharing the rigors of MIT education. I am obliged to my parents, Esphir and Gennady, for their support and motivation. Special thanks to my wife, Anna, for her love and patience, and for keeping me sane through the years of hard work and sacrifices.

This thesis was supported in part by the National Science Foundation (NSF) Collaboration in Mathematical Geosciences (CMG) grant ARC-0934404, and the Department of Energy (DOE) ISICLES initiative under LANL sub-contract 139843-1. Partial funding was provided by the department of Mechanical Engineering at MIT and 
by the Academic Programs Office at WHOI. My participation in the IMA "Large-scale Inverse Problems and Quantification of Uncertainty" workshop was partially funded by IMA NSF grants. 


\section{TABLE OF CONTENTS}

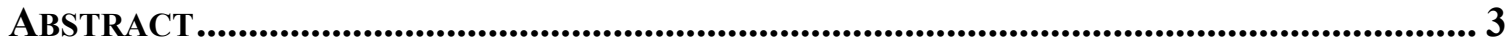

ACKNOWLEDGMENT

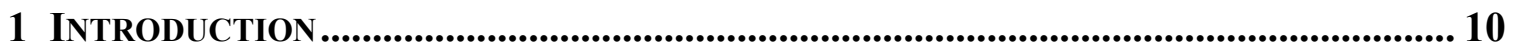

1.1 Uncertainty quantification in ocean state estimation ........................................ 10

1.2 Uncertainty of Drake Passage transport.............................................................. 15

1.3 Uncertainty assimilation framework for observation system design .................. 17

2 UNCERTAINTY QUANTIFICATION METHODOLOGY.................................................. 21

2.1 Inverse-predictive framework for ocean state estimation ................................. 21

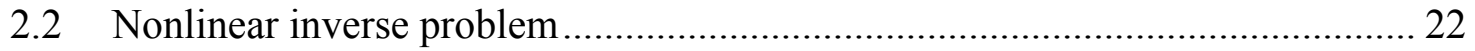

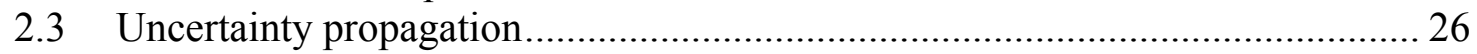

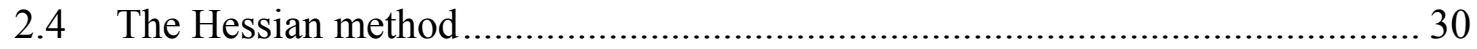

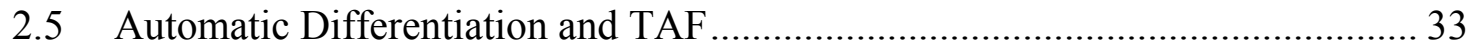

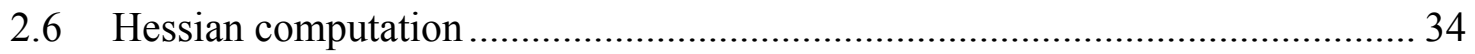

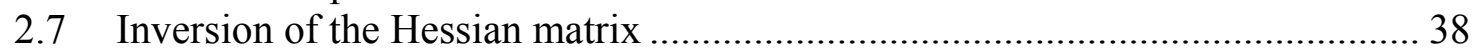

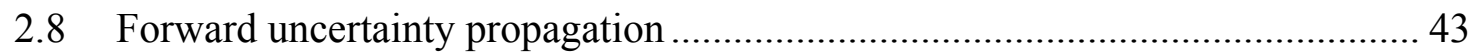

2.9 Summary of proposed UQ schemes ................................................................ 45

2.10 Synthetic UQ problem and the "identical twins" setup......................................46

2.11 Elementary illustration in two dimensions................................................... 47

2.12 Formalism for uncertainty quantification in ocean state estimation ...................50

3 Barotropic Models of ACC AND Flow through the DraKe PasSage............ 51

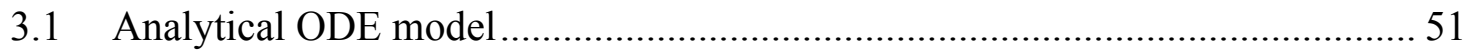

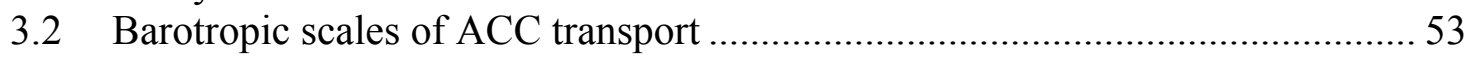

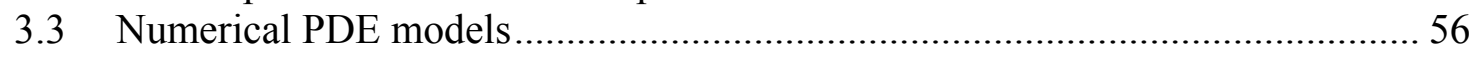

3.3.1 Idealized geometry - aqua planet ............................................................ 56

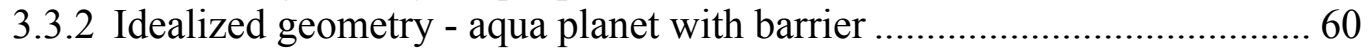

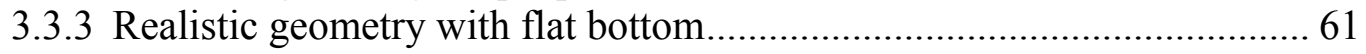

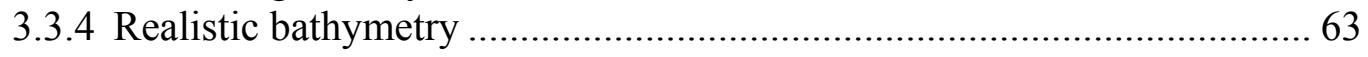

4 UQ ANALYSIS WITH ANALYTICAL ODE MODEL........................................................65

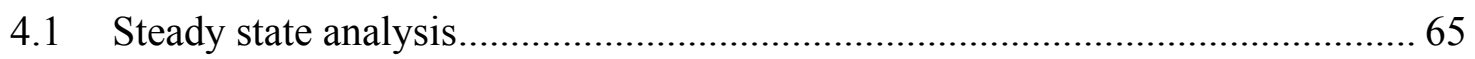

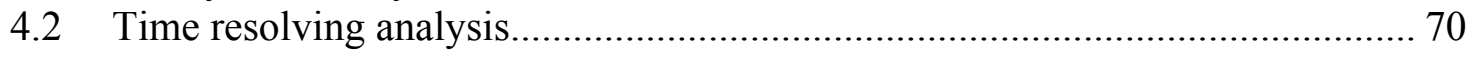

5 UQ ANALYSIS WITH MITGCM MODEL............................................................................ 78

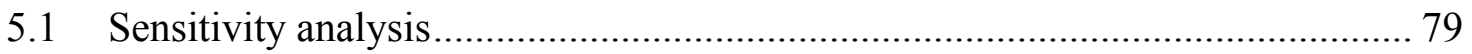

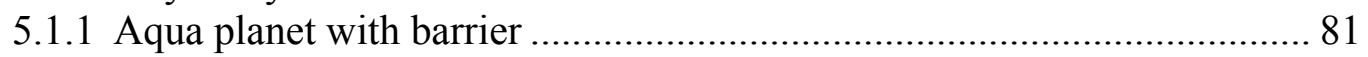

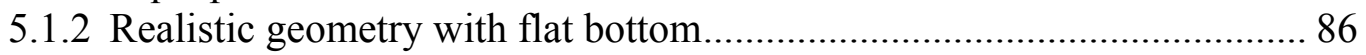

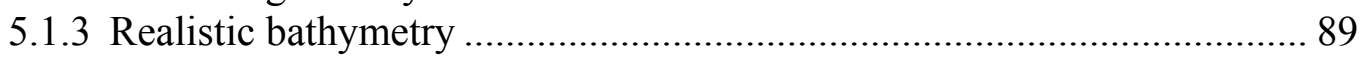

5.1.4 Time scales and evolution of sensitivity ………….................................. 91

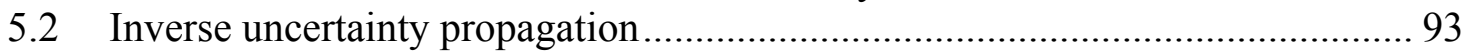

5.2.1 Uncertainty assimilation experiments.................................................... 93 
5.2.2 Hessian calculation and diagonalization ............................................ 95

5.2.3 Prior vs. posterior uncertainty: uncertainty reduction......................... 103

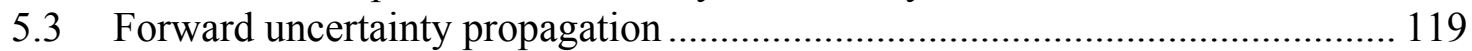

5.3.1 Realistic geometry with flat bottom............................................... 120

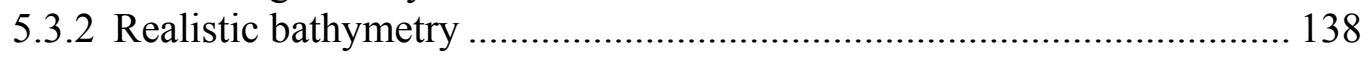

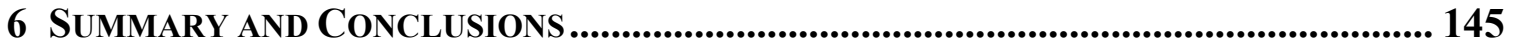

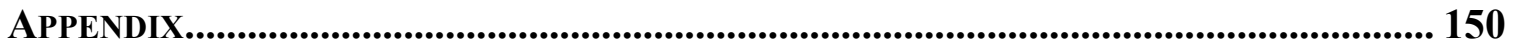

A. Maximum likelihood and Bayesian formulation of the inverse problem ......... 150

B. MITgem configuration details ............................................................ 151

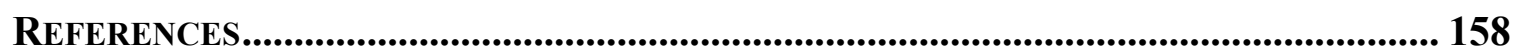


"All measurements, however careful and scientific, are subject to some uncertainties. Error analysis is the study and evaluation of these uncertainties, its two main functions being to allow the scientist to estimate how large his uncertainties are, and to help him to reduce them when necessary."

J. R. Taylor, 1982. An Introduction to Error Analysis: The Study of Uncertainties in Physical Measurements.

\section{Introduction}

\subsection{Uncertainty quantification in ocean state estimation}

Any quantitative description of ocean physics is subject to an inherent uncertainty since physical oceanography, being one of empirical physical sciences, is fundamentally based on measurement of the real oceans. Just as in any other branch of physics (Taylor 1982), analyzing the errors of ocean measurements and their effects on the uncertainty of the derived oceanographic description is a critical task. The ability to measure the actual ocean fields is limited not only by properties of a particular instrument but, importantly, due to the vast size of the observed system, spanning enormously large number of degrees of freedom. Additionally, oceanographic theory involves uncertainties due to inability to resolve the full spectrum of physical mechanisms involved as well as the fundamentally stochastic nature of the turbulent processes in the ocean. All quantitative ocean models, numerical and analytical, involve approximations or parameterizations, introducing uncertainty by selection of scales and parameters whose numerical value can be somewhat ambiguous. The computational methodologies involved in ocean modeling contribute to uncertainty due to truncation, finite precision and round-off errors. Furthermore, the applications of quantitative ocean description increasingly rely on the numerical values of computed physical variables, those range of validity must be understood. One of the primary such applications is the oceanic state estimation problem. It involves many of the sources of the uncertainty listed above. Therefore, quantification of this uncertainty and its effects on the confidence in the numerical values of the estimated ocean state is one of the primary tasks in applied oceanography.

The progress in modern computational ocean models and state estimation techniques has led to emergence of oceanographic state estimation as an applied methodology for 
detailed quantitative reconstruction of physically coherent ocean fields. "Estimating the Circulation and Climate of the Oceans" (ECCO) consortium is a large academic effort to produce dynamically and kinematically consistent estimate of time-evolving global ocean state by optimal combination of ocean general circulation model (GCM) with diverse ocean observations (see http://ecco-group.org). The practical mathematical framework for ECCO state estimation system is set up in Wunsch and Heimbach (2007). The approach is fundamentally different from sequential reanalysis techniques, which may introduce artificial imbalances in the time-evolving solution due to the causal form of data assimilation filtering schemes. These imbalances are eliminated in the ECCO estimation system by formally constraining the solution to obey the nonlinear GCM equations. The optimal fit of dynamical ocean model to the observational data is found by solving an unconstrained least-squares optimization problem formulated by the method of Lagrange multipliers. Gradient based iterative optimization schemes are implemented by recursive execution of forward and adjoint ocean models - a procedure known as the "adjoint method" solution. The major computational advantage of the adjoint state estimation methodology over sequential reanalysis is the elimination of the need to calculate and store large covariance matrices of the state vector. The computationally prohibitive size of covariance matrices in realistic ocean models is a key implementation weakness of sequential filtering and smoothing techniques, which must resort to approximate processing of covariances. However, the lack of covariance representation in the adjoint estimation procedure is a critical weakness of the Lagrange multipliers method. The implemented adjoint ocean state estimation systems (e.g. Losch and Heimbach 2007, Mazloff et al. 2010) lack error estimates or confidence bounds calculations, and do not currently allow to quantify the effects of observation uncertainty on the estimation.

The primary objective of this thesis is to advance the ECCO effort and improve ocean state estimation models by development of a feasible and scalable computational methodology for formal uncertainty quantification (UQ) for determination of model confidence and posterior error estimates. The need for formal uncertainty analysis in ocean state estimation is a well known outstanding challenge (e.g. Wunsch 2006). Mazloff et al. (2010) leave this effort for future work and instead base their quantitative measure of the estimated ocean state uncertainty on its resolved temporal standard 
deviations. While applicable to uncertainty estimation of steady state parameters perturbed by ergodic stationary random fluctuations, such approach imposes an unwarranted ergodicity on the estimated nonstationary ocean state (Forget and Wunsch 2007). Equating the temporal variability to the uncertainty of a nonstationary process discards the physics responsible for the dynamical evolution of the time-varying ocean state and it is not applicable for uncertainty quantification of time resolving ocean state estimation. Moreover, some confusion between the variability and the error statistics of ocean state estimates has led to controversies (Lermusiaux and Robinson 1999). The uncertainty of the estimated ocean state is a combination of dynamics predictability errors, assimilation of observations' uncertainty, and random model noise (Lermusiaux and Robinson 1999). A formal analysis of uncertainty in 4-dimensional state estimation should firstly provide a quantitative measure of the confidence in the computed state estimate. The goals of the developed UQ methodology also include: a quantitative insight into the underlying physical mechanisms of uncertainty, allowing an improved physical understanding of the dynamics of the estimated ocean state; and a procedure of posterior uncertainty analysis to combine the effects of assumed prior climatological uncertainties and the assimilated uncertainties of observations. Assimilating observation uncertainties into the ocean model allows model calibration by constraining its parameterizations and initial and boundary conditions. Furthermore, the analysis may be used in reverse to infer the required coverage and precision of observations needed to constrain the state estimation, leading to an objective procedure for future observation systems design.

Formal analysis of uncertainty estimates is possible in control theoretic framework (Wunsch and Heimbach 2007) through the Hessian of model-data misfit cost function, i.e. the matrix of second derivatives with respect to the model control parameters. The Hessian eigenvalues determine the principal curvature structures of the cost function. The curvature at the minimum point of the cost function is indicative of the uncertainty in the location of this point. Large curvatures indicate small posterior uncertainties and vise versa. Thus, the curvature information provides posterior error bounds through second order approximation of the control space geometry of the cost function. The idea to use the Hessians of model-data misfits to quantify the estimation uncertainty is not new and is standard in the field of nonlinear regression (e.g. Seber and Wild 2003). In the 
oceanographic context this idea was introduced by Thacker (1989), who described the application of the Hessian matrix for estimation of the precision of fitting linear oceanographic models to observations. He also heuristically suggested that "as long as the model is not too nonlinear, the inverse of the Hessian should provide a good approximation to the error-covariance matrix even in the nonlinear case". This heuristic linearized approach was continued in the nonlinear oceanographic uncertainty studies by Gunson and Malanotte-Rizzoli (1996) and Losch and Wunsch (2003). In the linearized framework of variational data assimilation (Moore et al. 2011) the inverse of the Hessian of the strictly quadratic misfit function can be formally related to the estimated error covariance matrix (see Section 2.2). Although it was also suggested (Lea et al. 2000) that for highly nonlinear chaotic systems these local derivatives based methods may not be appropriate, such situations have not been met so far in real oceanographic systems (Wunsch and Heimbach 2007).

Two main technical challenges which had prevented implementation of the aforementioned Hessian based uncertainty quantification system for realistic ocean state estimation are solved in this thesis - the size of the Hessian and the methodology for its calculation. The first technical challenge is due to the prohibitively large dimensionality of the state estimation space and therefore the Hessian matrix. The complexity of the multiscale ocean physics requires dynamics modeling with partial differential equations, involving nominally an infinity of degrees of freedom. Even after appropriate discretization the spatio-temporal ocean state comprises of a very large number of elements, e.g. $O\left(10^{9}\right)$ in Wunsch and Heimbach (2007) global ECCO configuration with one-degree and 23-level resolution, or $O\left(10^{13}\right)$ in a regional eddy resolving domain with 1/6-degree and 42 layer resolution (Mazloff et al. 2010). Limiting the degrees of freedom to model controls reduces the dimensionality, but it remains prohibitively large $-7 \cdot 10^{8}$ elements in (Mazloff et al. 2010) configuration. This "curse of dimensionality" (Oden et al. 2010, Wunsch 2006) is a well known problem, it is a challenge in the linear modeling framework and even more so in the nonlinear ocean state estimation problem. In this thesis we analyze the dimensionality of the Hessian and propose algorithms for its reduction. 
The second main technical challenge resolved in this thesis is computation of the Hessian. No machinery for its direct computation was previously available for large nonlinear ocean models. The previous efforts to calculate the Hessian were limited to linear (Thacker 1989) or linearized (Moore et al. 2011) models, small systems and approximate Hessian estimation techniques (Gunson and Malanotte-Rizzoli 1996, Losch and Wunsch 2003). Here, we develop a novel computational methodology for direct Hessian calculation of nonlinear GCMs with large dimensionality, scalable to realistic ocean state estimation problems. The key machinery for Hessian computation via direct differentiation of the MIT GCM code is based on the so-called automatic or algorithmic differentiation (AD) methodology for exact, up to floating point round-off, calculation of adjoint, Jacobian or Hessian derivative codes of arbitrary computer programs (Griewank and Walther 2008). The primary tool is Transformation of Algorithms in Fortran system (TAF) - a commercial successor of Tangent linear and Adjoint Model Compiler (TAMC) by Giering and Kaminski (1998), which was developed in parallel to and specifically targeted for the use in ECCO models. The central component of the ECCO adjoint method based state estimation system is the first derivative adjoint code of MITgcm, which was developed in a large collaborative effort at MIT over more than 10 years. The key technical challenge of this thesis was further pushing the edge the applied capability of TAF with development of a computationally feasible and scalable second derivative Hessian code of MITgcm. The development strategy was based on progressive application of TAF to a hierarchy of problems, starting with simple mathematically tractable models of increasing complexity, for validation and calibration of the method. The hierarchy of successfully implemented and analyzed models includes: generic linear transformation, damped harmonic oscillator, analytical idealized model of circumpolar flow, linear shallow water equations model, idealized and realistic barotropic configurations of MITgcm.

Other aspects of uncertainty quantification in ocean state estimation are resolved in this thesis. Sensitivity analyses have been proposed as a pathway to understanding the uncertainty in ocean models, as sensitivity responses to perturbation of model parameters can quantify the uncertainty of the system due to these parameters. Such uncertainty sensitivity calculations were suggested by Heimbach et al. (2011) by multiplying the 
sensitivity fields with either the expected uncertainties or the expected variability of model variables to quantify the contributions of these variables to the response of selected modeled quantities (e.g. the meridional volume transport). However, this intuitive connection of sensitivity and uncertainty was not put in a formal statistical context and correlations of variability or uncertainty were not taken into account. Here, we formalize the relation between sensitivity and uncertainty analysis frameworks and clarify the role of sensitivities in forward and inverse uncertainty propagation. Another open question raised in Heimbach et al. (2011) is the relationship between optimal patterns of singular perturbations (Zanna et al. 2010) and expected uncertainty patterns. The developed UQ methodology here explains this relationship in terms of eigenvectors of the Hessian matrix.

\subsection{Uncertainty of Drake Passage transport}

The physical problem of interest in this thesis is the dynamics of Antarctic Circumpolar Current (ACC), focusing on Drake Passage transport and the formal analysis of its uncertainty. The physics of ACC is a complex problem, which is different from the typical basin scale dynamics due to lack of continuous meridional boundaries which constrain ocean flows in other basins. The ACC is the largest current in the global ocean and has a unique impact on ocean and climate dynamics. Although the ACC transports large amounts of water, heat and salt in the zonal direction, it acts as a dynamic barrier to the meridional transport. This isolates the waters surrounding the Antarctic continent from the global ocean and is therefore believed to explain the cold Antarctic climate. To leading order ACC dynamics resemble that of the atmospheric jet stream - governed by geostrophic and thermal wind balances, while the Sverdrup balance does not hold. The physical mechanisms governing the ACC are not completely understood and are a subject of an active debate (Rintoul et al. 2001, Olbers et al. 2004), the available observations are scarce relative to its large geographic extent (Meredith et al. 2011) and ocean state estimation is necessary to reconstruct its detailed structure (Mazloff et al. 2010). In this thesis we apply the developed ocean state estimation UQ methodology to the problem of ACC reconstruction. Our goals are twofold, to demonstrate the developed methodology 
and to attempt contributing to the understanding of ACC. We expect that quantifying the sensitivity of ACC dynamics and the uncertainty of its estimate can shed more light on the physics of this complex system and help better explain its effects on deep ocean circulation and global climate.

Drake Passage is the narrowest constriction of ACC and therefore is a natural place to quantify its strength. The rate of flow through the Passage is believed to determine water mass budgets and circulation patterns in the global ocean (Meredith et al. 2011). The attempts to quantify it from the available sparse and uncertain measurements lead to large disagreements. One of the first hydrographic-section-based estimates of ACC transport was $110 \mathrm{~Sv}$ (Clowes 1933). This value is believed to be relevant today (Meredith et al 2011), given the range of measured estimates from $5 \mathrm{~Sv}$ westward to $237 \mathrm{~Sv}$ eastwards and the understanding that the transport fluctuates in response to varying forcing fields (Whitworth and Peterson 1985, Weijer and Gille 2005). The Drake Passage transport is also a standard diagnostic of many numerical global ocean models, with simulated values spread over a wide range from well under $100 \mathrm{~Sv}$ to well over $200 \mathrm{~Sv}$ (Olbers et al. 2004). The reasons for this uncertainty are not fully understood but are believed to be dependent on the uncertainty in modeling of wind forcing and thermohaline processes, as well as on model numerics, resolution, representation of topography and parameterization of subgrid processes. Our goal is to demonstrate the proposed UQ methodology by analyzing the uncertainty of the modeled ACC transport. In order to keep the size of the UQ problem and its computational requirements manageable in the early state of methodology development, we focus on low resolution models with simplified physics. These highly idealized models capture only some essential mechanisms and simplify the uncertainty analysis by reducing the complexity of the real oceanographic system.

Our idealized ACC models are designed to resolve the uncertainty of circumpolar transport to complement and compare to its estimated variability. The state of the art estimate of ACC transport and its variability is given by the Southern Ocean State Estimation (SOSE) solution of Mazloff, Heimbach and Wunsch (2010). The fluctuations of the transport over a period of 2 years are shown in Figure 1.1. The estimate of the transport is $153 \pm 5 \mathrm{~Sv}$, given by the temporal average and the temporal standard deviation. The mean transport, the upper and the lower variability bounds are displayed 
in the figure. The variability range of $10 \mathrm{~Sv}$ is added to the figure as an error bar (on the right). Note that this variability measure is constant in time and does not reflect the changing state of the transport dynamics, which is weaker in winter months (southern hemisphere summer) and stronger in the summer. It also does not explicitly reflect observation and control variable uncertainties. We develop a formal uncertainty measure which, in contrast, is rooted in the dynamics of the system and varies as function of time evolving dynamically in response to external forcing. The figure shows that the transport variability fluctuations appear on synoptic time scales. We wish to resolve the uncertainty due to the mechanisms governing this variability. It has been established (Wearn and Baker 1980, Withworth 1983, Meredith et al. 2011) that the subannual variability is mainly barotropic. Accordingly, we will focus on the uncertainty of the barotropic ACC mechanisms. The subannual fluctuations are driven by wind stress and steered by bottom topography (Weijer and Gille 2005). These processes are described by the linear theory of wind driven circumpolar transport (LaCasce and Isachsen 2010), which guides our idealized barotropic ACC modeling framework.

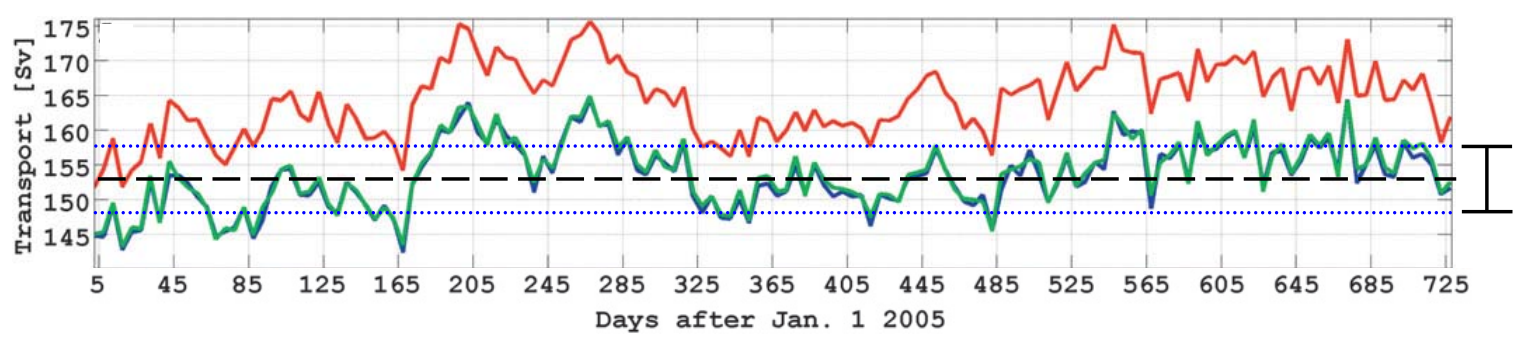

Figure 1.1: Estimated ACC transport at different meridional cross-sections from Mazloff et al. (2010). The lower line (blue solid) shows the transport at the Drake Passage over the period of two years. The time average and the standard deviation of this transport are shown with dashed and dotted lines. Error bars corresponding to twice the standard deviation are shown on the right of the figure.

\subsection{Uncertainty assimilation framework for observation system design}

The key oceanographic goals in development of uncertainty quantification methodology for practical ocean state estimation system are: assimilation of ocean observations uncertainty in the dynamical model, an objective physics-based evaluation of observation system contribution to ocean monitoring and quantitative foundation for optimal ocean 
observation systems design. The sources of uncertainty in ocean state estimation system may be separated to internal modeling errors, e.g. due to physical representation or computational restrictions, and to external uncertainties of various model inputs propagating through the model. The main focus of this dissertation is on the latter, quantifying the assimilated observation uncertainty and its effects on the propagation of the uncertainties in model's initial and boundary conditions as well as other model control parameters.

It is important to understand the difference between traditional error propagation calculations in forward modeling settings and the UQ requirements of the inverse modeling framework of ocean state estimation. Unlike the traditional approach (Taylor 1982), which is based on application of the given forward model or its first derivative to project model input perturbations to model outputs, the quantification of inverse model uncertainty requires uncertainty assimilation machinery, which calculates the effects of the uncertainties in the assimilated observations on the estimated ocean state. The uncertainty assimilation machinery is based on the inverse model, which is not available explicitly, the assimilated observations are the output of the forward model and the estimated state is the input. Therefore, it is the perturbations of ocean model output that need to be projected to model input, which makes UQ for inverse modeling more complex.

Design of ocean observation systems for sustained ocean monitoring at the Drake Passage is an important task in oceanographic and climate research where "strategic improvements could be made concerning how this is conducted" (Meredith et al. 2011). In this dissertation we propose a quantitative framework for optimal ocean observation systems design, using the example of barotropic ACC transport monitoring at the Drake Passage. The large uncertainty in the previously reported observational estimates of the ACC transport (see Section 1.2) warrants the development of an objective methodology for design of new ocean observation systems intended to constrain that uncertainty. An optimally designed oceanographic monitoring system can be purposed to reduce observation uncertainty by location of the sensors in the most sensitive points. Efficient ocean observation system may be designed to optimally complement the existing moorings, repeat hydrography lines and the deployed remote sensing instruments. The 
qualitative effort to design the future ocean observation systems based on understanding of ocean dynamics, its resolution and estimation uncertainty is reflected in the objectives of the modern Drake Passage monitoring campaigns. The World Ocean Circulation Experiment (WOCE) primary goals included determining the "representativeness" of WOCE data sets (Meredith et al. 2011) obtained at the Drake Passage with bottom pressure recorders (BPRs) and repeat hydrography lines. The cDrake experiment observations (Meredith et al. 2011) with the array of bottom-moored Current and Pressure-Recording Inverted Echo Sounders (CPIES) spanning the Drake Passage, intended for assimilation in SOSE and evaluation of uncertainty of dynamical estimates, are planned to be used in guiding future monitoring systems. It is one of the goals of this thesis to provide a quantitative framework for these efforts utilizing the UQ and the observation uncertainty assimilation methodology designed for the ocean state estimation system.

The idea of putting the sensors in the most sensitive locations is an intuitive one, because large sensitivity amplifies the effects of uncertainty of the measurements on the estimated variables and reducing this measurement uncertainty has the largest impact on constraining the target variables. Such approach is straightforward in forward modeling framework, where the inputs to the model can be directly measured and forward model sensitivity is defined as the derivative of model output with respect to model inputs. However, if a quantitative estimate of the achieved uncertainty reduction is required, one must remember that uncertainties add up not linearly but in quadrature (Taylor 1982) and need to account for sensitivity coupling due to uncertainty correlation. Moreover, the algebra of uncertainty quantification in the inverse modeling framework of ocean state estimation is considerably more complicated. The sensitivities still play a central role but involve nonlinear combinations of derivatives of two separate models. In the inverse modeling framework the measurements are the output of the ocean model, while the model inputs are given by adjustable model controls, which are not directly observed but are constrained to minimize the misfit between the model outputs and the observations. The calculation of the sensitivity of the estimated oceanographic variables to the observations requires, then, evaluation of the sensitivity of model inputs (controls) to model outputs (observations) and its combination with the sensitivity of a second ocean 
model, projecting the observations-constrained model controls to the estimated oceanographic target variables. Although the first step of the above is possible for fully observable and invertible systems by application of inverse function theorem, the inversion of the sensitivity matrix is not generally possible for the non-observable noninvertible ocean models. More elaborate analysis is required for quantification of the reduction of uncertainty in ocean observation system design, which can be placed in the framework of observation uncertainty assimilation developed in this dissertation. It will be shown how the different sensitivities represented by the derivatives of the two separate models are combined in quadratic structures extending the notion of the quadrature rule for uncertainties. For nonlinear systems, the first derivative sensitivities are complemented by the second derivative fields representing the sensitivities of sensitivities. These "second order sensitivities" represent the curvature of the ocean model in its range space. Both, the quadratic sensitivity structures and the second order sensitivities are the consequences of the proposed Hessian UQ and uncertainty assimilation methodology. The thesis below formalizes all these statements and develops quantitative foundations for objective observation system design. 


\section{Uncertainty Quantification Methodology}

\subsection{Inverse-predictive framework for ocean state estimation}

We develop a unified inverse-predictive state estimation methodology for large ocean models by combination of inverse and predictive modeling frameworks ${ }^{1}$. The inverse model estimates the ocean state variables and the uncertainty of these estimates from supplied observational data and observation uncertainty. In a dynamical systems setting the inverse model estimates the variables that control the state of the ocean as computed by a forward model. The vector of the estimated state or control variables, denoted $\mathbf{x}$, can include physical ocean variables, geometrical system parameters, any combination of initial and boundary conditions and forcing fields, or other parameters of models describing the dynamical system ${ }^{2}$. We define a forward model $\boldsymbol{M}$, generally nonlinear, that maps these control variables to model counterparts of the observed data vector $\mathbf{y}$

$$
\mathbf{y}=\mathbf{M}(\mathbf{x}) .
$$

If model $\boldsymbol{M}$ is invertible the solution to the inverse problem can be written as

$$
\mathbf{x}=\boldsymbol{M}^{-1}(\mathbf{y}) \text {. }
$$

In general when the overall modeling goal is to estimate some target quantities which are not among the model control variables $\mathbf{x}$, then another nonlinear forward model $N$, either prognostic or diagnostic, can be used to map the estimated ocean state controls to the desired target quantities $\mathbf{z}$

$$
\mathbf{z}=N(\mathbf{x}) .
$$

Our inverse-predictive two-step state estimation method first applies inverse modeling to assimilate observations in a dynamical ocean model to find optimal model controls and to constrain their a priori known or assumed uncertainties. Then, the optimized model is used to evaluate the target quantities $\mathbf{z}$ and the a posteriori estimated uncertainty of the

\footnotetext{
${ }^{1}$ Similar approach was introduced by Kaminski et al. (2010) in Gaussian statistical setting in the context of quantitative network design and implemented for only 57 control variables.

${ }^{2}$ To simplify the derivation here we do not explicitly distinguish between the state $\mathbf{x}(t)$, initial conditions $\mathbf{x}(0)$ and unknown controls $\mathbf{u}(t)$ in the notation of Wunsch and Heimbach (2007) and as suggested therein absorb them all in a generalized control vector $\mathbf{x}$. We also disregard the explicit time dependence of the state $\mathbf{x}(t)$, considering the entire time domain all at once in the state vector $\mathbf{x}$. This notation allows single derivation to apply for both static (time-independent) and time-evolving models.
} 
controls is propagated forward to obtain the posterior uncertainty estimate for the target model predictions. The developed framework also allows comparing the posterior and the prior uncertainty estimates of the target variables $\mathbf{z}$, which quantifies the constraint of target uncertainties by assimilation of observation uncertainty.

For invertible model $\boldsymbol{M}$ the inverse-predictive process for the estimated quantities can be written formally as

$$
\mathbf{z}=N\left(M^{-1}(\mathbf{y})\right) .
$$

Schematically, the workflow of the inverse-predictive algorithm can be expressed for the estimated quantities and their uncertainties (here marked temporarily by symbol $\Delta$ ) as

$$
\begin{aligned}
\mathbf{y} & \rightarrow \mathbf{x} \rightarrow \mathbf{z} \\
\Delta \mathbf{y} & \rightarrow \Delta \mathbf{x} \rightarrow \Delta \mathbf{z}
\end{aligned}
$$

The following sections formulate the algebra and the computational machinery for implementation of such inverse-predictive procedure for large ocean state estimation problems. Formal definitions for uncertainty of multivariate variables are introduced in Section 2.3.

\subsection{Nonlinear inverse problem}

A general nonlinear forward model is not always formally invertible or its inverse is not directly available, preventing application of (2.1.2). Therefore, we frame solution of the inverse problem as minimization a quadratic cost function given by a weighted $\mathrm{L}_{2}$ norm of model-data misfit vector in the range space of the forward model $\boldsymbol{M}$. The value of the optimal argument of the cost function in model's domain, denoted $\hat{\mathbf{x}}$, is the estimate of the solution to the inverse problem. For fully invertible models with adequate data the value of the misfit function at this optimum is expected to be zero. Explicitly, we define the cost function

$$
J_{1}(\mathbf{x})=\frac{1}{2}(\boldsymbol{M}(\mathbf{x})-\mathbf{y})^{T} \mathbf{W}^{-1}(\boldsymbol{M}(\mathbf{x})-\mathbf{y})
$$

where $\mathbf{y}$ represents the measured data and $\mathbf{W}$ is a symmetric and positive-definite weighting matrix, such that for $\hat{\mathbf{x}}$ satisfying $J_{1}(\hat{\mathbf{x}})=0$, necessarily $M(\hat{\mathbf{x}})=\mathbf{y}$. 
In practice, the available data will typically be noisy and we have to rewrite the data equation (2.1.1) with an additive ${ }^{3}$ noise vector $\mathbf{n}$

$$
\mathbf{y}=\boldsymbol{M}(\mathbf{x})+\mathbf{n}
$$

When noisy data is substituted into the cost function, its minimum may not reach zero and is interpreted as the least (weighted) sum of squares of model-data misfits. The optimum $\hat{\mathbf{x}}$ is then the least-squares solution of the inverse problem. This method of solution of nonlinear inverse problem is known as the "method of total inversion" (Wunsch 2006 p. 173) and is a specific case of the general least-squares method. In general least-squares the weighting in the quadratic cost function can be any matrix, not necessarily symmetric and positive-definite (Wunsch 2006 p. 54). Also, in least-squares methods it is customary to add to the cost function a weighted regularization term

$$
J_{0}(\mathbf{x})=\frac{1}{2}\left(\mathbf{x}-\mathbf{x}_{0}\right)^{T} \mathbf{S}^{-1}\left(\mathbf{x}-\mathbf{x}_{0}\right)
$$

nudging the solution towards some a priori prescribed value $\mathbf{x}_{0}$. The effect of adding the regularization term is improving conditioning of the inversion and reducing variance of the solution at the expense of introducing some bias (Wunsch 2006 p. 55).

In many applications it is customary to put a deterministic inverse problem in a statistical setting and render the least-squares solution in the form of linear minimum variance estimation by choosing the above weighting matrices be a priori assumed dispersion covariance matrices of noise and control vectors. Specifically, model-data misfits are weighted by second moment matrix of noise

$$
\mathbf{W}=\mathbf{R} \equiv\left\langle\mathbf{n n}^{\mathrm{T}}\right\rangle
$$

which is equal the covariance matrix of noise, i.e. $\mathbf{C}_{\mathbf{n} \mathbf{n}} \equiv\left\langle(\mathbf{n}-\langle\mathbf{n}\rangle)(\mathbf{n}-\langle\mathbf{n}\rangle)^{\mathrm{T}}\right\rangle$, if noise is assumed zero mean process $\langle\mathbf{n}\rangle=0$. The regularization term is weighted by the dispersion covariance of controls $\mathbf{x}$ around the prior assumed controls value $\mathbf{x}_{0}$

\footnotetext{
${ }^{3}$ We note that the assumption of additive noise, as the explicit notation suggests here, appears to be only a special case for a general nonlinear estimation problem. In simple linear regression it is customary to assign the model-data misfits a meaning of additive observation noise. More general linear regression allows also "errors in variables" (Wunsch 2006 p. 171), leading to a nonlinear estimation problem and its solution by the method of total least-squares. However, in nonlinear regression the formalism of the method of total inversion allows combining parameterized model errors with model-data misfits and augmenting the regressors vector to form a generalized solution vector $\mathbf{x}$. Thus, our framework includes both - the additive measurement noise and other noise represented by the model errors, without contradicting the derivation of the quadratic cost function term (2.2.1) weighting only the additive noise components (see Wunsch 2006 eq. 3.62).
} 


$$
\mathbf{S}=\mathbf{P}_{0} \equiv\left\langle\left(\mathbf{x}-\mathbf{x}_{0}\right)\left(\mathbf{x}-\mathbf{x}_{0}\right)^{\mathrm{T}}\right\rangle
$$

This dispersion covariance matrix is equal to the prior covariance of the controls if their prior value is also the mean $\mathbf{x}_{0}=\langle\mathbf{x}\rangle$. The combined cost function is written as

$$
J(\mathbf{x})=\frac{1}{2}\left[(\boldsymbol{M}(\mathbf{x})-\mathbf{y})^{T} \mathbf{R}^{-1}(\boldsymbol{M}(\mathbf{x})-\mathbf{y})+\left(\mathbf{x}-\mathbf{x}_{0}\right)^{T} \mathbf{P}_{0}^{-1}\left(\mathbf{x}-\mathbf{x}_{0}\right)\right]
$$

If the forward model $\boldsymbol{M}$ is only weakly nonlinear and its first order Taylor expansion is a valid approximation throughout the optimization domain, the model can be expressed in terms of its Jacobian transformation ${ }^{4} \partial \boldsymbol{M} / \partial \mathbf{x}^{T}$

$$
\boldsymbol{M}(\mathbf{x})=\boldsymbol{M}\left(\mathbf{x}_{0}\right)+\frac{\partial \boldsymbol{M}}{\partial \mathbf{x}^{T}}\left(\mathbf{x}-\mathbf{x}_{0}\right)+O\left(\left\|\mathbf{x}-\mathbf{x}_{0}\right\|^{2}\right)
$$

which is known as tangent linear approximation. Within the tangent linear region the inverse problem is solved by a linear transformation given by a closed form analytical expression. To derive it, note that at the minimum of the cost function its gradient is zero

$$
\nabla J(\hat{\mathbf{x}})=\left(\frac{\partial \boldsymbol{M}}{\partial \mathbf{x}^{T}}\right)^{T} \mathbf{R}^{-1}(\boldsymbol{M}(\hat{\mathbf{x}})-\mathbf{y})+\mathbf{P}_{0}^{-1}\left(\hat{\mathbf{x}}-\mathbf{x}_{0}\right)=0 .
$$

Substituting the tangent linear expansion (2.2.7) into (2.2.8) and neglecting higher than first order terms yields

$$
\left[\left(\frac{\partial \boldsymbol{M}}{\partial \mathbf{x}^{T}}\right)^{T} \mathbf{R}^{-1}\left(\frac{\partial \boldsymbol{M}}{\partial \mathbf{x}^{T}}\right)+\mathbf{P}_{0}^{-1}\right]\left(\hat{\mathbf{x}}-\mathbf{x}_{0}\right)=\left(\frac{\partial \boldsymbol{M}}{\partial \mathbf{x}^{T}}\right)^{T} \mathbf{R}^{-1}\left(\mathbf{y}-\boldsymbol{M}\left(\mathbf{x}_{0}\right)\right)
$$

which can be rearranged to form the linear inversion operator

$$
\hat{\mathbf{x}}=\mathbf{x}_{0}+\left[\left(\frac{\partial \boldsymbol{M}}{\partial \mathbf{x}^{T}}\right)^{T} \mathbf{R}^{-1}\left(\frac{\partial \boldsymbol{M}}{\partial \mathbf{x}^{T}}\right)+\mathbf{P}_{0}^{-1}\right]^{-1}\left(\frac{\partial \boldsymbol{M}}{\partial \mathbf{x}^{T}}\right)^{T} \mathbf{R}^{-1}\left(\mathbf{y}-\boldsymbol{M}\left(\mathbf{x}_{0}\right)\right) .
$$

This solves the linearized inverse problem in the tangent linear domain.

Another combination of the same two equations (2.2.7) and (2.2.8) yields an expression for the error covariance matrix of the solution - the dispersion of estimate $\hat{\mathbf{x}}$ around its unknown true value $\mathbf{x}$, defined

$$
\mathbf{P}=\left\langle(\hat{\mathbf{x}}-\mathbf{x})(\hat{\mathbf{x}}-\mathbf{x})^{T}\right\rangle
$$

\footnotetext{
${ }^{4}$ We define the Jacobian transformation as the linear transformation given by the Jacobian matrix (Rogers 1980, p. 35). Its transpose is called the gradient (Magnus and Neudecker 1988, p. 87), expressed in the standard notation (Seber and Wild 2003) $\nabla \boldsymbol{M}(\mathbf{x}) \equiv \partial \boldsymbol{M} / \partial \mathbf{x}=\left(\partial \boldsymbol{M} / \partial \mathbf{x}^{T}\right)^{T}$ in agreement with Wunsch (2006, p. 27).
} 
This requires rewriting (2.2.7) by expanding the forward model at the solution $\boldsymbol{M}(\hat{\mathbf{x}})$ around the true value $\mathbf{x}$. Substituting the modified tangent linear expansion into zero gradient (2.2.8) condition yields

$$
\left[\left(\frac{\partial \boldsymbol{M}}{\partial \mathbf{x}^{T}}\right)^{T} \mathbf{R}^{-1}\left(\frac{\partial \boldsymbol{M}}{\partial \mathbf{x}^{T}}\right)+\mathbf{P}_{0}^{-1}\right](\hat{\mathbf{x}}-\mathbf{x})=\left(\frac{\partial \boldsymbol{M}}{\partial \mathbf{x}^{T}}\right)^{T} \mathbf{R}^{-1}(\mathbf{y}-\boldsymbol{M}(\mathbf{x}))+\mathbf{P}_{0}^{-1}\left(\mathbf{x}_{0}-\mathbf{x}\right)
$$

Multiplying (2.2.12) on the right by its transpose and taking the expectation yields

$$
\begin{aligned}
& {\left[\left(\frac{\partial \boldsymbol{M}}{\partial \mathbf{x}^{T}}\right)^{T} \mathbf{R}^{-1}\left(\frac{\partial \boldsymbol{M}}{\partial \mathbf{x}^{T}}\right)+\mathbf{P}_{0}^{-1}\right]\left\langle(\hat{\mathbf{x}}-\mathbf{x})(\hat{\mathbf{x}}-\mathbf{x})^{T}\right\rangle\left[\left(\frac{\partial \boldsymbol{M}}{\partial \mathbf{x}^{T}}\right)^{T} \mathbf{R}^{-1}\left(\frac{\partial \boldsymbol{M}}{\partial \mathbf{x}^{T}}\right)+\mathbf{P}_{0}^{-1}\right]^{T}=} \\
& =\left(\frac{\partial \boldsymbol{M}}{\partial \mathbf{x}^{T}}\right)^{T} \mathbf{R}^{-1}\left\langle(\mathbf{y}-\boldsymbol{M}(\mathbf{x}))(\mathbf{y}-\boldsymbol{M}(\mathbf{x}))^{T}\right\rangle \mathbf{R}^{-T}\left(\frac{\partial \boldsymbol{M}}{\partial \mathbf{x}^{T}}\right)+\mathbf{P}_{0}^{-1}\left\langle\left(\mathbf{x}_{0}-\mathbf{x}\right)\left(\mathbf{x}_{0}-\mathbf{x}\right)^{T}\right\rangle \mathbf{P}_{0}^{-T}
\end{aligned}
$$

where two additional cross terms proportional to $\left\langle\left(\mathbf{x}_{0}-\mathbf{x}\right)(\mathbf{y}-\boldsymbol{M}(\mathbf{x}))^{T}\right\rangle$ and its transpose were cancelled out by assuming that observation noise is not correlated with prior dispersion of the controls. Using definitions (2.2.4) and (2.2.5) and the symmetry of the matrices, the right hand side of (2.2.13) simplifies to

$$
\left(\frac{\partial \boldsymbol{M}}{\partial \mathbf{x}^{T}}\right)^{T} \mathbf{R}^{-1} \mathbf{R R}^{-1}\left(\frac{\partial \boldsymbol{M}}{\partial \mathbf{x}^{T}}\right)+\mathbf{P}_{0}^{-1} \mathbf{P}_{0} \mathbf{P}_{0}^{-1}=\left(\frac{\partial \boldsymbol{M}}{\partial \mathbf{x}^{T}}\right)^{T} \mathbf{R}^{-1}\left(\frac{\partial \boldsymbol{M}}{\partial \mathbf{x}^{T}}\right)+\mathbf{P}_{0}^{-1}
$$

which can be cancelled out with the left hand side of (2.2.13) to yield

$$
\mathbf{P}=\left[\left(\frac{\partial \boldsymbol{M}}{\partial \mathbf{x}^{T}}\right)^{T} \mathbf{R}^{-1}\left(\frac{\partial \boldsymbol{M}}{\partial \mathbf{x}^{T}}\right)+\mathbf{P}_{0}^{-1}\right]^{-1}
$$

Both results (2.2.10) and (2.2.15) can be equivalently derived by application of the Gauss-Markov theorem, as shown explicitly by Gunson and Malanotte-Rizzoli (1996) and discussed further by Wunsch (2006, p. 129) for the case of a linear forward model. Note, that while the results are identical, the derivations differ - the Gauss-Markov theorem minimizes the dispersion of the solution, while we minimize the modelobservation misfits. The advantage of the Gauss-Markov minimum variance estimation approach is that the resulting error covariance of the solution (2.2.15) is ensured to be minimal among all possible linear inversion operators. The disadvantages, however, stem from the assumptions of the Gauss-Markov theorem, limited to only linear inversion operators and the tangent linear domain of the forward model. We focus instead on a more general nonlinear inverse problem framework of the method of total inversion. The 
nonlinear least-squares problem is solved with the adjoint method by iterative gradient based minimization of the cost function, which is not limited by the domain of local tangent linear approximation. The uncertainty of the nonlinear solution is quantified with the Hessian method, based on the quadratic expansion of the cost function at the solution point (Wunsch 2006, eq. 3.68; see also Thacker 1989). We have shown above that in the tangent linear limit (2.2.15) our method is equivalent to the linear (Gauss-Markov) error estimate. Nonetheless, the method extends also to the nonlinear case and provides a local quantitative error measure. For details of the adjoint method for solution of nonlinear inverse problem we refer the reader to Wunsch and Heimbach (2007) and Heimbach (2008) MITgcm user guide. The focus of this thesis is on uncertainty quantification and propagation methodology accompanying the adjoint method.

\subsection{Uncertainty propagation}

We define uncertainty of a solution estimate $\hat{\mathbf{x}}$ by its error covariance matrix (2.2.11), i.e. its dispersion matrix around its unknown true value $\mathbf{x}$. For linear invertible models both forward and inverse propagation of uncertainty have closed form expressions and are straightforward to derive. Consider a linear model given by matrix $\mathbf{M}$

The model input uncertainty is

$$
\mathbf{y}=\mathbf{M x}
$$

$$
\mathbf{P}_{\mathbf{x x}}=\left\langle(\hat{\mathbf{x}}-\mathbf{x})(\hat{\mathbf{x}}-\mathbf{x})^{T}\right\rangle
$$

which can be projected to the model output by forward transformation

$$
\mathbf{P}_{\mathbf{y y}}=\left\langle(\hat{\mathbf{y}}-\mathbf{y})(\hat{\mathbf{y}}-\mathbf{y})^{T}\right\rangle=\left\langle(\mathbf{M} \hat{\mathbf{x}}-\mathbf{M x})(\mathbf{M} \hat{\mathbf{x}}-\mathbf{M x})^{T}\right\rangle=\mathbf{M} \mathbf{P}_{\mathbf{x x}} \mathbf{M}^{T}
$$

known as matrix congruence for invertible models ${ }^{5}$. The backward transformation is derived by direct inversion of (2.3.3)

$$
\mathbf{P}_{\mathbf{x x}}=\mathbf{M}^{-1} \mathbf{M} \mathbf{P}_{\mathbf{x x}} \mathbf{M}^{T} \mathbf{M}^{-T}=\mathbf{M}^{-1} \mathbf{P}_{\mathbf{y y}} \mathbf{M}^{-T}=\left(\mathbf{M}^{T} \mathbf{P}_{\mathbf{y y}}^{-1} \mathbf{M}\right)^{-1}
$$

Note, that the last RHS form is convenient because it does not require explicit use of the inverse model $\mathbf{M}^{-1}$, but only of two matrix inverse operations. The inverse of error

\footnotetext{
${ }^{5}$ Matrix congruence is a change of basis transformation and it preserves the number of positive, negative and zero eigenvalues of the matrix. The definition of congruence depends on the existence of the inverse matrix $\mathbf{M}^{-1}$. Congruence transformation is a generalization of a similarity transformation for matrices $\mathbf{M}$ which are not necessarily unitary.
} 
covariance or uncertainty matrix can be termed information matrix. Accordingly, we interpret the inverse uncertainty propagation transformation as a backward projection of the information matrix and then its inversion. This form is also interesting because it suggests extending the framework of inverse uncertainty propagation to models which are not formally invertible. Since only the adjoint of the model is required for back projection of the information matrix, it can be applied even if the matrix $\mathbf{M}^{-1}$ does not exist.

For linear invertible models the forward and inverse propagation transformations (2.3.3) and (2.3.4) can be combined in our inverse-predictive state estimation framework. The solution equation for target variables (2.1.4) rewritten for the linear models $\mathbf{M}$ and $\mathbf{N}$ is

$$
\mathbf{z}=\mathbf{N M}^{-1} \mathbf{y}
$$

The accompanying uncertainty propagation formula is

$$
\mathbf{P}_{\mathrm{zz}}=\mathbf{N}\left(\mathbf{M}^{T} \mathbf{P}_{\mathrm{yy}}^{-1} \mathbf{M}\right)^{-1} \mathbf{N}^{T}
$$

These two expressions complete the formulation of our UQ algorithm (2.1.5) and (2.1.6) for the linear invertible case. The general case with models that may be not invertible or nonlinear is more complex.

If the linear forward model $\mathbf{M}$ is not invertible the back projected information matrix $\mathbf{M}^{T} \mathbf{P}_{\mathbf{y y}}^{-1} \mathbf{M}$ is singular. The matrix cannot be inverted, which prevents the application of the RHS of (2.3.4) for inverse propagation of uncertainty. In practice even for an invertible model $\mathbf{M}$ the information matrix may be nearly singular and the required matrix inversion be poorly conditioned. Such singularity or high matrix condition number frequently arise if some components of the solution vector $\mathbf{x}$ are poorly determined by the data or there is high disparity between levels of uncertainty in different components. A standard solution in both ill-conditioned cases is to add a small regularization matrix $\mathbf{Q}$ to ensure the well-posedness of the matrix inversion, which leads to approximation of the uncertainty of the solution:

$$
\mathbf{P}_{\mathbf{x x}} \approx\left(\mathbf{M}^{T} \mathbf{P}_{\mathbf{y y}}^{-1} \mathbf{M}+\mathbf{Q}^{-1}\right)^{-1}
$$

The resulting form is identical to the uncertainty of the regularized least-squares solution (2.2.15). Thus, the addition of the regularization term (2.2.3) has two related but separate 
meanings: nudging the solution to a prior value and controlling the matrix inversion in

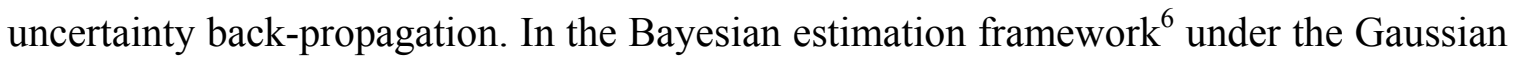
assumption, this regularization term has a formal interpretation as the negative logarithm of the prior probability of the forward model inputs $\mathbf{x}$. In that framework the regularization matrix $\mathbf{Q}$ is the prior covariance of the inputs $\mathbf{x}$, denoted $\mathbf{P}_{0}$. Moreover, the application of the non-regularized uncertainty assimilation formula (2.3.4) quantifies the error dispersion of maximum likelihood estimates, while the regularized version (2.3.7) calculates the posterior covariance of inputs $\mathbf{x}$. Therefore, the application of (2.3.7) can be considered a prior-to-posterior transformation of uncertainty, which is also consistent with the least-squares result (2.2.15).

For proper positive-definite covariance matrices, this transformation of prior uncertainty to posterior uncertainty is always an uncertainty reduction. To show this we rewrite the RHS of (2.3.7) utilizing the matrix inversion lemma ${ }^{7}$

$$
\left(\mathbf{M}^{T} \mathbf{P}_{\mathbf{y y}}^{-1} \mathbf{M}+\mathbf{Q}^{-1}\right)^{-1}=\mathbf{Q}-\mathbf{Q} \mathbf{M}^{T}\left(\mathbf{P}_{\mathbf{y y}}+\mathbf{M} \mathbf{Q} \mathbf{M}^{T}\right)^{-1} \mathbf{M} \mathbf{Q}
$$

The second term on the RHS is the difference between the prior and the posterior covariances. It is a positive-definite matrix, called the posterior uncertainty reduction matrix $\boldsymbol{\Gamma}$ in Chapter 4. This posterior uncertainty reduction is interpreted as constraining the uncertainty of model inputs by the observations $\mathbf{y}$ and their uncertainty $\mathbf{P}_{\mathbf{y y}}$. In fact, (2.3.8) shows that for linear models this uncertainty reduction calculation is independent of the values of the observed data and thus can be performed before the actual data is available. This decoupling of data and uncertainty assimilation calculations suggest using data independent UQ analysis for design of observational systems with desired uncertainty properties of the estimated state. Extending such UQ based experimental design approach to nonlinear ocean models requires extending the presented uncertainty propagation algebra to nonlinear models, as discussed below. Decoupling of data and uncertainty assimilation calculations for nonlinear models is considered in Section 2.10.

\footnotetext{
${ }^{6}$ Details of the equivalent derivation in the Bayesian framework are given in Appendix A.

${ }^{7}$ The matrix inversion lemma, also known as Woodbury matrix identity or Sherman-Morrison-Woodbury formula, is the exact matrix identity $(A+U C V)^{-1}=A^{-1}-A^{-1} U\left(C^{-1}+V A^{-1} U\right)^{-1} V A^{-1}$
} 
For nonlinear forward models, when the input uncertainty is not too large relative to the range of validity of its tangent linear expansion, the uncertainty can be propagated forward by the tangent linear model. Consider the nonlinear target model $N(\mathbf{x})(2.1 .3)$, which we expand about the true value of model controls

$$
N(\hat{\mathbf{x}})=N(\mathbf{x})+\frac{\partial N}{\partial \mathbf{x}^{T}}(\hat{\mathbf{x}}-\mathbf{x})+O\left(\|\hat{\mathbf{x}}-\mathbf{x}\|^{2}\right)
$$

and substitute the expansion into the definition of target variable uncertainty, neglecting higher than first order terms:

$$
\mathbf{P}_{\mathrm{zz}}=\left\langle(N(\hat{\mathbf{x}})-N(\mathbf{x}))(N(\hat{\mathbf{x}})-N(\mathbf{x}))^{T}\right\rangle=\left(\frac{\partial N}{\partial \mathbf{x}^{T}}\right) \mathbf{P}_{\mathbf{x x}}\left(\frac{\partial N}{\partial \mathbf{x}^{T}}\right)^{T}
$$

The resulting forward uncertainty transformation is identical to (2.3.3), with the linear forward model replaced by the tangent linear model given by the Jacobian matrix $\partial \boldsymbol{N} / \partial \mathbf{x}^{T}$. It is important to note, that the range of validity of (2.3.10) depends on the range of the tangent linear approximation of the target model (2.3.9) and needs to be tested in applications.

We can propagate forward either posterior or prior uncertainty of $\mathbf{x}$. This allows to compare the prior and posterior uncertainty of target variables $\mathbf{z}$ in our inverse-predictive state estimation framework. Explicitly, returning to notation of (2.2.15) and restricting to only scalar target variable $z$, the prior estimate of the target and its uncertainty are given by

$$
z_{0}=N\left(\mathbf{x}_{0}\right), \quad \sigma_{z_{0}}^{2}=\left(\frac{\partial N}{\partial \mathbf{x}^{T}}\right) \mathbf{P}_{0}\left(\frac{\partial N}{\partial \mathbf{x}^{T}}\right)^{T}
$$

The posterior target estimate and its uncertainty are

$$
z=N(\mathbf{x}), \quad \sigma_{z}^{2}=\left(\frac{\partial N}{\partial \mathbf{x}^{T}}\right) \mathbf{P}\left(\frac{\partial N}{\partial \mathbf{x}^{T}}\right)^{T}
$$

The posterior uncertainty of the target variable is always smaller than its prior uncertainty.

Before completing the discussion of forward uncertainty propagation we note that the matrix form of (2.3.12) can also be written out explicitly in the scalar form:

$$
\sigma_{z}^{2}=\sum_{i}\left(\frac{\partial N}{\partial x_{i}}\right)^{2} \sigma_{x_{i}}^{2}+\sum_{i} \sum_{j \neq i} \frac{\partial N}{\partial x_{i}} \frac{\partial N}{\partial x_{j}} \sigma_{x_{i}} \sigma_{x_{j}} \rho_{i j}
$$


Here $\sigma_{x i}$ are the standard deviations of the controls and $\rho_{i j}$ are the correlation coefficients. The first sum includes only the contributions of the diagonal terms of the covariance of controls, representing the propagation of the uncorrelated standard deviations. The double summation of the off-diagonal covariance terms is the contribution of the correlations in the uncertainties of the controls. If the correlation uncertainty terms are zero, then the uncertainty propagation formula (2.3.13) reduces to the familiar addition in quadrature rule for independent uncertainties (Taylor 1982).

Propagation of uncertainty in the inverse direction for nonlinear models is more involved and is developed in the separate section below.

\subsection{The Hessian method}

For a linear version of the forward model $\boldsymbol{M}$ the cost function (2.2.6) is quadratic in the control variable vector $\mathbf{x}$. Its quadraticity or its convexity properties are defined by its Hessian matrix, i.e. the matrix of second derivatives

$$
\mathbf{H}_{J}(\mathbf{x}) \equiv \frac{\partial^{2} J}{\partial \mathbf{x}^{2}}
$$

The matrix is independent of the value of $\mathbf{x}$ where the derivative is calculated, i.e. it's a fixed global characteristic of the estimation problem. For a nonlinear model $\boldsymbol{M}$ in general the cost function is not necessarily globally quadratic, but for sufficiently smooth functions there exists a local range around any $\mathbf{x}$ where $J$ is approximately quadratic and its curvature is described by the Hessian matrix. In the vicinity of its minimum $\hat{\mathbf{x}}$ the cost function can be approximated, to second order, by the quadratic form

$$
J(\mathbf{x}) \approx \frac{1}{2}(\mathbf{x}-\hat{\mathbf{x}})^{T} \mathbf{H}_{J}(\hat{\mathbf{x}})(\mathbf{x}-\hat{\mathbf{x}}),
$$

where $\mathbf{H}_{J}(\hat{\mathbf{x}})$ is the Hessian matrix evaluated at $\hat{\mathbf{x}}$ and the gradient of $J$ vanishes (2.2.8). The analytical expression for the Hessian of (2.2.6) at $\hat{\mathbf{x}}$ is (Thacker 1989, Seber and Wild 2003)

$$
\mathbf{H}_{J}(\hat{\mathbf{x}})=\left(\frac{\partial \boldsymbol{M}}{\partial \mathbf{x}^{T}}\right)^{T} \mathbf{R}^{-1}\left(\frac{\partial \boldsymbol{M}}{\partial \mathbf{x}^{T}}\right)+\left(\frac{\partial^{2} \boldsymbol{M}}{\partial \mathbf{x}^{2}}\right)^{T} \mathbf{R}^{-1}(\boldsymbol{M}(\hat{\mathbf{x}})-\mathbf{y})+\mathbf{P}_{0}^{-1}
$$

The first term is the backward projection of the inverse covariance of observation uncertainty, similar to (2.3.4) if the linear forward model $\mathbf{M}$ is substituted with the 
tangent linear model of $\boldsymbol{M}$. This first term is commonly referred to as the linearized Hessian and was calculated in the previous studies of error covariance inversion (Thacker 1989, Gunson and Malanotte-Rizzoli 1996, Losch and Wunsch 2003). The second term is the product of the tensor of second derivatives of the multivariate model $\boldsymbol{M}$, i.e. the collection of the Hessian matrices of each of its scalar components (Thacker 1989), with the inverse covariance of observations and with the residual misfits vector. This second term is named the nonlinear term (Gunson and Malanotte-Rizzoli 1996). It appears only for nonlinear model $\boldsymbol{M}$, since for linear model $\mathbf{M}$ the tensor of its second derivatives is zero. The third term is the contribution of the quadratic regularization term (2.2.3) and is equal, depending on the context, to the inverse of the weighting or the regularization matrix. In the Bayesian context it is the inverse of the prior covariance matrix.

If a perfect solution $\hat{\mathbf{x}}$ is found, such that the vector of the residual misfits $(\boldsymbol{M}(\hat{\mathbf{x}})-\mathbf{y})$ vanishes at the solution, or if the forward model $\boldsymbol{M}$ is linear, then the second term disappears and the Hessian matrix is equal to the inverse of the posterior error covariance of the solution (2.2.15). In these two cases the calculation of the Hessian matrix and its inversion complete the inverse uncertainty propagation. However, in general the residual misfits do not vanish, in particular if the regularization term is non zero at the solution point - as can be seen in the optimum condition equation (2.2.8), and which is leading to regularization bias. Moreover, in the ocean state estimation problem the models of interest are nonlinear. Therefore, the Hessian matrix in general is different from the inverse of the linear error covariance and the difference is the nonlinear Hessian term. It can be argued that in some problems this difference can be neglected, when the residual misfits are small or the nonlinearity of the forward model is relatively weak. In other cases this nonlinear term can be large and should not be ignored. These are known as large-residual problems and are discussed in Chapter 14 of Seber and Wild (2003). In the ocean modeling context, Gunson and Malanotte-Rizzoli (1996) attempted to estimate the effect of this nonlinear term by separately calculating and comparing the full Hessian to the linearized Hessian. They found that while the normalized Frobenius norm of the difference was $7 \%$, the nonlinear term introduced singularity to otherwise well conditioned linearized Hessian by making its condition number a very large. They proceeded by neglecting the nonlinear term and relating only the linearized Hessian to the 
error covariance of the solution, relying on the linear Gauss-Markov theory. Their required assumption had to be that the nonlinear model is approximately linear globally over the domain of optimization, which is not the case in general. Similarly, Losch and Wunsch (2003) estimated only the linearized Hessian and interpreted its inverse as the covariance of the estimation solution, regardless of the magnitude of residual.

To quantify the uncertainty of the solution of a nonlinear state estimation problem solved with the adjoint method consider the local quadratic expansion of the cost function (2.4.2). If the nonlinear model is sufficiently smooth, the local Hessian of the cost function describes its local curvature and within this local range quantifies the dispersion of estimation errors. As was shown, in the linear limit or for well-posed invertible models the inverse of the Hessian approximates the posterior error covariance

$$
\mathbf{P} \approx \mathbf{H}_{J}(\hat{\mathbf{x}})^{-1},
$$

which is a global measure of uncertainty dispersion. For general models we can define the inverse of the Hessian matrix as the local quadratic measure of uncertainty, and apply same analysis as typically done for error covariance matrices without adding the global interpretation to the resulting uncertainty estimates. Since any symmetric positive semi-definite matrix is a covariance matrix of some random vector, any inverse Hessian estimated at a convex ${ }^{8}$ point is a covariance matrix. Thus the local quadratic uncertainty measure can be interpreted as the locally equivalent error covariance, formally extending uncertainty covariance analysis to nonlinear models.

A rigorous theoretical foundation for use of local Hessians for uncertainty quantification in estimation problem is available in the Bayesian and Information Theory frameworks. The Hessian can be interpreted as the observed Fisher information matrix (Efron and Hinkley 1978) and its inverse is the Cramer-Rao bound on variance of the estimated control variables. However, these details fall beyond the scope of the current thesis and are left to be addressed in future work. To avoid the possible issues due to the difference between the full and the linearized Hessians in this thesis we will focus on UQ

\footnotetext{
${ }^{8}$ Convex point here means - not a saddle point, and thus might include zero curvature directions. Of course the Hessian in such case would not be directly invertible, its inversion would be defined in terms of its pseudo-inverse.
} 
implementation for zero-residual problems using the method of identical twins (Losch and Wunsch 2003), see Section 2.10 below.

\subsection{Automatic Differentiation and TAF}

The computational ocean state estimation system is implemented in numerical ECCO environment (MITgcm documentation Chapter 8, Heimbach 2008), with its dynamical ocean modeling kernel provided by MITgem (Marshall et al. 1997a,b, http://mitgcm.org/). The source code of the numerical model, written in Fortran 77, together with integrated precompiled external libraries, is referred to as the forward model code. We generate the derivatives of the forward code, namely the Hessian, the Jacobian and the adjoint codes with the Automatic Differentiation (AD) tool - TAF (Transformation of Algorithms in Fortran). Overview of TAF can be found in Giering and Kaminski (1998) and in TAF manual (available from FastOpt.com). A heuristic concise but insightful introduction to TAF predecessor TAMC (Tangent linear and Adjoint Model Compiler) with illustration of its oceanographic application appears in Marotzke et al. (1999). A comprehensive explanation of TAF application in MITgcm for sensitivity and state estimation studies is given in Chapter 5 of MITgcm documentation (Heimbach 2008), with the mathematical framework and theoretical background laid out in Wunsch and Heimbach (2007). Here we avoid repeating the details of implementation of $\mathrm{AD}$ machinery and only highlight the main challenges of its application for uncertainty quantification codes.

The central difficulty with $\mathrm{AD}$ application to large and complex codes is that, despite what the name implies, the procedure is not fully automatic. For simple short codes application of AD machinery is straightforward and was verified by complete UQ system implementation for generic linear transformation, damped harmonic linear oscillator and the analytical ACC model (see Chapter 4). In contrast, differentiation of MITgcm requires manual programming of code flow directives to help the differentiating compiler process the code. Selected subroutines require manual differentiation, external precompiled libraries are hidden and bypassed, some loop restructuring is needed to design recursive variable storage and recomputation solutions leading to checkpointing schemes. These are the regular challenges in adjoint model development, which required 
several man-years of coding by the MITgcm development team. The unique challenge in the Hessian code generation is the repeated differentiation of first derivative AD output code. For large codes, writing the directives to facilitate processing of the machine generated first derivative code and differentiate it second time is even more complicated. A specific difficulty encountered and resolved was differentiating of the checkpointing storage constructs in adjoint code, while preserving the checkpointing efficiency. Therefore, practical applications of $\mathrm{AD}$ to complex codes should be considered only semi-automatic.

\subsection{Hessian computation}

The Hessian matrix of the cost function can be estimated in several different ways. The Hessian is defined as the matrix of second derivatives (2.4.1) or equivalently, as the derivative of the gradient, i.e. the Jacobian matrix derivative of the gradient treated as a vector function. Therefore, it can be computed by differentiating the gradient (adjoint) code - either via $\mathrm{AD}$ or column by column by finite differences of the gradient. Alternatively, Hessian finite difference schemes can be derived directly in terms of the perturbed cost function. We implemented a simple forward difference Hessian scheme

$$
\frac{\partial^{2} J}{\partial x_{i} \partial x_{j}}=\frac{J\left(x+h_{i} \hat{e}_{i}+h_{j} \hat{e}_{j}\right)-J\left(x+h_{i} \hat{e}_{i}\right)-J\left(x+h_{j} \hat{e}_{j}\right)+J(x)}{h_{i} h_{j}}
$$

Here $h_{i}, h_{j}$ are the magnitudes of finite perturbations of the independent variables, the unit vectors $\hat{e}_{i}, \hat{e}_{j}$ represent the perturbed components. This scheme is slow to converge and is computationally expensive. More accurate higher order finite difference schemes have even heavier computational load. Therefore, finite difference schemes are not scalable and cannot be applied for computation of large Hessian matrices. We applied this scheme only sparsely for validation of our $\mathrm{AD}$ results and implemented it in the HESSIAN_CHECK subroutine.

Application of TAF for semi-automatic calculation of the Hessian allows several different options. Direct differentiation of the gradient code with TAF allows one to choose between calculation of the full matrix, its few columns or its left or right product with an arbitrary column or row vector. For multivariate functions with only one 
dependent (output) variable the most computationally efficient strategy for evaluation of the second derivative is the so-called forward-over-reverse mode (TAF manual, p. 18). Therefore, we first apply TAF to the forward model in reverse mode to obtain the adjoint code, then TAF is applied in forward mode to generate the Hessian code of the cost function. This procedure reduces the computational complexity of the resulting Hessian code because tangent linear differentiation of the adjoint code preserves the efficiency of checkpointing schemes. That second application of TAF can be done either in vector mode - to generate the entire matrix in single run, or in scalar mode - which for each run produces a single Hessian-vector right product. The later leads to either column by column evaluation of the full Hessian matrix or can be used inside Lanczos algorithm ${ }^{9}$ (Trefethen and Bau 1997) for iterative eigenanalysis of the Hessian without actually constructing or storing the full matrix, which can both be prohibitively large. For example, in double precision the size of our $80 * 180 * 6=86400$ elements long control vector is $675 \mathrm{~KB}$, while the size the Hessian is $56 \mathrm{~GB}$, preventing its storage in the random-access memory of standard computers. Moreover, the run time for a single Hessian-vector product operation in our implementation is $O(10$ to 600 seconds), depending on the duration of the simulation (see Table 2.1). It would require from 10 days to more than a year of computer run time for a single evaluation of the whole Hessian matrix. Accordingly, our UQ methodology for very large systems cannot practically rely on construction of the Hessian matrix, which leads to the development of a matrix-free reduced rank Hessian UQ algorithm in the next section.

\begin{tabular}{|l|c|c|c|}
\hline & FWD & AD & HESS \\
\hline CPU time (seconds) 90 days run & 37 & $168-213$ & $375-426$ \\
\hline CPU time (seconds) 30 days run & 12.5 & $55-71$ & $129-146$ \\
\hline CPU ratio (FWD) & 1 & $4.4-5.8$ & $10.1-11.6$ \\
\hline CPU ratio (AD) & & 1 & $2.0-2.3$ \\
\hline
\end{tabular}

Table 2.1 Code execution duration (user CPU time) for different simulated time periods (90 and 30 days) and computational complexity factors ratios (number of timesteps scaling) relative to the forward (FWD) and the adjoint (AD) codes. See text for explanation of the ranges shown for the adjoint and the Hessian (HESS) codes. The codes were executed on Dual Core AMD Opteron(tm) Processor 875 (2210 MHz, cache size: $1024 \mathrm{~KB}$ ).

\footnotetext{
${ }^{9}$ Lanczos algorithm is based on the power method projection to Krylov subspace. We implement the Lanczos algorithm with the implicitly restarted Arnoldi method library - ARPACK (Lehoucq et al. 1998), using its symmetric matrix version, known as the Lanczos iteration (Trefethen and Bau 1997).
} 
We briefly analyze the computational complexity and temporal scalability of the derivative codes. Table 2.1 lists the execution times for two representative simulation period configurations - 90 and 30 days runs. For the adjoint and the Hessian codes a range of execution times is shown. The lower computational load is observed for the synthetic, zero residual, identical twins configurations (see Section 2.10). The higher load is for the regular configurations with non-zero misfits. The difference can be explained by the runtime simplification of the executed code for trivial (zero) variable values, when the program reaches recursive iteration loops. The analysis has indicated that the execution time scales linearly with simulation length for all three codes, i.e. the computational complexity is $O(N)$, where $N$ is the number of simulation timesteps. The factors of the linear scaling are shown in terms of their ratios relative to the forward and the adjoint codes execution times. That is, if the forward model runtime is $m_{\mathrm{fwd}}{ }^{\circ} N$ and the adjoint model runtime is $m_{\mathrm{ad}} N$, then the table lists the ratio of the complexity factors $m_{\mathrm{ad}} / m_{\mathrm{fwd}}$. These ratios are equivalent to the ratios of user CPU times and the results are consistent with Giering et al. (2005), who obtain the ratios 5.5 and 11 for the adjoint and the Hessian codes relative to the forward code. Note, that in Giering et al. (2005) the derivative codes were generated with three-level checkpointing, while our implementation uses two-level checkpointing only. The presented analysis focused on simulation time scalability only. The complete scalability and computational complexity analysis requires also spatial resolution scaling as well as the separate effects of different control fields, which is left for future work.

In this thesis we follow the presented $\mathrm{AD}$ approaches and compute the Hessians either entirely or only few columns depending on their size. Our AD Hessian computations do not involve linearization, approximation or truncation errors and were verified with analytical calculations. Although our direct Hessian computation approach is the preferable way for estimating the Hessians, the alternative Hessian estimation techniques are worth a brief examination. Although they only approximate the actual Hessian, through their analytical form or simplified numerics they allow a better understanding of the computed Hessian structure. The structure of the Hessian reflects the convexity of the estimation problem, understanding of which provides a critical insight into the dynamical properties of the model and the estimated uncertainty. 
It is useful to reexamine the structure of the analytical expression of the Hessian of model-data misfit function, here given without the regularization term:

$$
\mathbf{H}(\hat{\mathbf{x}})=\left(\frac{\partial \boldsymbol{M}}{\partial \mathbf{x}^{T}}\right)^{T} \mathbf{R}^{-1}\left(\frac{\partial \boldsymbol{M}}{\partial \mathbf{x}^{T}}\right)+\left(\frac{\partial^{2} \boldsymbol{M}}{\partial \mathbf{x}^{2}}\right)^{T} \mathbf{R}^{-1}(\boldsymbol{M}(\hat{\mathbf{x}})-\mathbf{y}) .
$$

The first term is the inner product of model Jacobian transformations weighted by the covariance of observations. Equivalently, it is the weighted outer product of model gradients. It is independent of second derivatives and is constant for linear models, i.e. independent of point $\hat{\mathbf{x}}$ were it is evaluated. The second term is the product of the model Hessian tensor with model-data misfit vector normalized by the data covariance. This term is zero for linear models (model Hessian vanishes) or when the inverse problem has an exact solution (zero misfit). These insights contribute to understanding of the properties of the Hessian. For linear or invertible models, the Hessian and therefore the solution uncertainty are completely determined by the model Jacobian. The first term the linearized Hessian, may dominate the structure of the Hessian also in more general cases, when a nonlinear model is nearly invertible or its nonlinearity is weak. The second term captures the uncertainty structure due to large nonlinearity of the model. Furthermore, this form of the Hessian does not include a regularization term. It is the form of the Hessian for the pure inverse (maximum likelihood) problem and therefore defines directly the nullspace and the observability properties of the system analyzed. Addition of the prior term regularizes the Hessian inversion, but unless it is based on known prior statistics it is only an artificial computational construct which introduces a bogus bias masking the assimilated physical information. Addition of the regularization term should, desirably, be based on understanding of the original structure of the nullspace of the physical system and the regularization matrix should be designed to minimize solution bias and masking of the physically meaningful uncertainty subspaces. For this reason, we prefer to design a prior-independent UQ algorithm, as detailed in the following sections.

The linearized Hessian matrix

$$
\mathbf{H}_{L}=\left(\frac{\partial \boldsymbol{M}}{\partial \mathbf{x}^{T}}\right)^{T} \mathbf{R}^{-1}\left(\frac{\partial \boldsymbol{M}}{\partial \mathbf{x}^{T}}\right)
$$


can be calculated using only the model Jacobian code, as was done by Thacker (1989) and Losch and Wunsch (2003), which eliminates the need for the Hessian code. Moreover, the form of the linearized Hessian has an important theoretical connection to singular value analysis of the sensitivity matrix (Zanna et al. 2010), because the eigenvectors of the linearized Hessian are the rescaled singular vectors of the Jacobian matrix. Understanding this connection is the pathway to exploring "the relationship between optimal patterns and expected uncertainty patterns", the question raised in (Heimbach et al. 2011).

Alternatively, a low rank approximation of the Hessian can be obtained with only the adjoint code, that is the Jacobian code of the scalar cost function. The Hessian is constructed iteratively from the cost function gradients computed in quasi-Newton minimization algorithm (Veerse 1999).

Ultimately, in this thesis we are interested in the inverse of the Hessian matrix and its forward propagation for large nonlinear ocean models. The discussions above lead us to formulation of reduced rank inversion and forward propagation schemes for high dimensional systems, presented in next sections. Remaining research questions include the possibility of using exact $\mathrm{AD}$ Hessians in ocean state estimation optimization algorithms as well as normalization and scaling of the numerical implementation of Hessians to limit the round off errors. The Hessian can also guide a theoretical scaling of the inverse problem, or alternatively be used as a preconditioner for the optimization schemes.

\subsection{Inversion of the Hessian matrix}

For small to medium dimension problems, up to $O\left(10^{3}-10^{4}\right)$ elements, the Hessian matrix can be directly inverted by dense matrix algebra methods. We implemented direct inversion based on LU factorization using a sequence of LAPACK library procedures DGETRF and DGETRI. This direct approach cannot be applied when the Hessian matrix is ill conditioned, i.e. when the ratio of its largest to smallest eigenvalue is large. Due to limitations of finite precision numerics, computer round-off errors make dense algebra calculations of ill-conditioned inverse useless even if the inverse formally exists. 
Nonetheless, the inverse can be constructed explicitly using a pseudo-inverse matrix form based on the orthogonal spectral expansion that always exists for symmetric matrices:

$$
\begin{aligned}
\mathbf{H} & =\mathbf{V} \boldsymbol{\Lambda} \mathbf{V}^{T}=\sum_{i} \lambda_{i} \mathbf{v}_{i} \mathbf{v}_{i}^{T} \\
\mathbf{H}^{+} & =\mathbf{V} \boldsymbol{\Lambda}^{-1} \mathbf{V}^{T}=\sum_{i} \frac{1}{\lambda_{i}} \mathbf{v}_{i} \mathbf{v}_{i}^{T}
\end{aligned}
$$

Here $\lambda_{i}$ and $\mathbf{v}_{i}$ are Hessian eigenvalues and eigenvectors, $\boldsymbol{\Lambda}$ is diagonal matrix, $\mathbf{V}$ is the orthogonal matrix of eigenvectors (its columns). Each outer product of eigenvectors, $\mathbf{v}_{i} \mathbf{v}_{i}{ }^{T}$, is a rank-1 matrix. The pseudo-inverse is the linear combination of these rank-1 matrices weighted by the reciprocals of the Hessian eigenvalues (see also Thacker 1989). For invertible (nonsingular) matrices the pseudo-inverse is equal to the dense (exact) inverse. For singular or nearly singular matrices it is possible to construct a reduced rank pseudo-inverse by omitting zero or small eigenvalues of the Hessian and corresponding eigenvectors from (2.7.2), which is equivalent to just setting the corresponding diagonal terms of $\boldsymbol{\Lambda}^{-1}$ to zero. These omitted eigenvectors correspond to control variable combinations that are poorly constrained by the data or lie in the nullspace of the problem, their eigenvalues representing the small or zero information gained. The reciprocals of Hessian eigenvalues represent the uncertainty variances of the constrained control variables combinations, thus the uncertainty of the omitted eigenvectors is very large. At the limit when the Hessian is singular, its inverse does not exist and, as can be seen explicitly in (2.7.2) - the full pseudo-inverse would be infinite. The interpretation of the reduced pseudo-inverse then is - omitting the nullspace and contracting the assimilated uncertainty covariance only to the data-supported sub-domain in the control parameters space.

An alternative to partial inversion of ill-conditioned Hessian is to slightly modify its structure to suppress its nullspace by providing plausible constraints, which make otherwise zero eigenvalues finite. This is achieved by augmenting the model-data misfit cost function with a penalty term quadratic in control parameters' deviation from a prescribed prior value (Losch and Wunsch 2003). The curvature of the quadratic term is given by a coefficient matrix (2.2.3), which specifies the strength of the constraints reducing the uncertainty of the corresponding eigenvectors. For linear models this 
method is known as generalized Tikhonov regularization and it renders the inverse problem one of "tapered least squares" (Wunsch 2006, p. 55). In a Bayesian framework the same penalty term is produced by multiplication by a Gaussian prior probability density. In the context of matrix inversion, the regularization term is just a matrix of the same dimensionality as the original matrix that is added to it before application of inversion algorithm. Since in all these cases the regularization may introduce bias to the pure inverse, it is preferable to keep the magnitude of the regularization terms small meaning large prior uncertainty. There is a certain arbitrariness in the selection of the regularization term, unless the value of the prior uncertainty is known with high confidence. Even within the Bayesian framework, unless there is a strong evidence for a particular prior distribution, the choice of the prior can be questioned and pure inversion is preferred whenever is possible.

For small ill-posed inverse problems regularization of the Hessian matrix is a practical solution as it then can be directly inverted either with dense or spectral pseudo-inverse methods. Losch and Wunsch (2003) demonstrate regularization, suppression of effective (numerical) null space, and reduction of condition number from $O\left(10^{8}\right)$ to $O(10)$ for 160x160 Hessian. For large problems, however, regularization can be counterproductive as the resulting well-conditioned matrix is both too large to be inverted by a dense method and would require full spectral decomposition for construction of the pseudoinverse. Such full spectral decomposition would be computationally prohibitive for very large matrices even with matrix-free iterative methods, such as the Lanczos algorithm. Moreover, if regularization is not applied we can utilize the sparsity of the original illconditioned Hessian and its null space structure. If the Hessian is formally singular or has a numerical null space, its spectral decomposition (2.7.1) can be reduced by considering only the nonzero eigenvalues. In this case only the corresponding eigenvectors need to be evaluated for construction of (2.7.1) or (2.7.2). One way to proceed then would be to construct a reduced rank pseudo-inverse and keep in mind that it does not constrain the full control space, leaving infinite uncertainty for some control parameters combinations belonging to the problem's nullspace. This solution is satisfactory for the analysis of the uncertainty structure of the inverse problem, for understanding its dynamics and can be applied for optimal experimental design. It cannot, however, be used in our inverse- 
predictive state estimation framework, as our forward propagation transformation is not defined for partial uncertainty matrix. In other words, the remaining unconstrained control parameter combinations would propagate forward with complete uncertainty about some aspects of the estimated state, rendering the target variables completely uncertain.

Nevertheless, another solution is possible for large inverse problems by combining the advantages of both of the above techniques - the reduced rank and the regularization approaches to Hessian inversion. The solution is based on application of the matrix inversion lemma and is inspired by the Gauss-Markov or the Bayesian solution for posterior uncertainty reduction (2.3.8). We recall that the equation for the reduced posterior covariance is given by inversion of the sum of inverse prior covariance and the back projection of the inverse covariance of misfits

$$
\mathbf{C}_{\text {Post }}^{-1}=\mathbf{C}_{\text {Prior }}^{-1}+\mathbf{M}^{T} \mathbf{R}^{-1} \mathbf{M}
$$

The matrix inversion lemma permits instead of explicitly inverting the posterior covariance, which typically is large and in general is not sparse, to get the same result by inverting the sum of the covariance of misfits and the forward projection of the prior covariance, both of which would typically have much smaller dimension as well as be sparse

$$
\mathbf{C}_{\text {Post }}=\mathbf{C}_{\text {Prior }}-\mathbf{C}_{\text {Prior }} \mathbf{M}^{T}\left(\mathbf{R}+\mathbf{M} \mathbf{C}_{\text {Prior }} \mathbf{M}^{T}\right)^{-1} \mathbf{M C}_{\text {Prior }}
$$

The proposed solution is derived by noting that the same lemma can be applied, instead to the back projection of the misfit information, to the Hessian written in the diagonalized form as a matrix product of its eigenvalues and eigenvectors $\mathbf{H}=\mathbf{V} \mathbf{\Lambda} \mathbf{V}^{T}$

$$
\begin{gathered}
\mathbf{P}^{-1}=\mathbf{P}_{0}^{-1}+\mathbf{V} \mathbf{\Lambda} \mathbf{V}^{T} \\
\mathbf{P}=\mathbf{P}_{0}-\mathbf{P}_{0} \mathbf{V}\left(\boldsymbol{\Lambda}^{-1}+\mathbf{V}^{T} \mathbf{P}_{0} \mathbf{V}\right)^{-1} \mathbf{V}^{T} \mathbf{P}_{0}
\end{gathered}
$$

Note that as long as the eigendecomposition was precise, no other approximation was made at this stage. Nonetheless, even a precise eigendecomposition can reduce the dimensionality of the calculation as the Hessian may not have full rank, reducing the number of columns in the eigenvector matrix $\mathbf{V}$ and the size of eigenvalue matrix $\boldsymbol{\Lambda}$ to number of the nonzero Hessian eigenvalues. In the limiting case when the rank of the 
Hessian is one, the matrix $\mathbf{V}$ is a single column vector, $\boldsymbol{\Lambda}$ is a scalar and the only inversion involved is that of a scalar. The latter case has the same form as the case of a linearized Hessian of only single scalar observed data. This lossless computational compression is a major advantage of the proposed approach: while the traditional solution (2.7.4) reduces the required matrix inversion computations from the dimensionality of the control space to that of the observations space, (2.7.6) further reduces the inversion computations to dimensionality of only the independent observations which add non zero information to uncertainty constraint in the control space, equal to the rank of the Hessian.

$$
\operatorname{dim}(\mathbf{P}) \geq \operatorname{dim}(\mathbf{R}) \geq \operatorname{dim}(\boldsymbol{\Lambda})=\operatorname{rank}(\mathbf{H})
$$

The inversion of the resulting smaller information matrix $\Lambda^{-1}+\mathbf{V}^{T} \mathbf{P}_{0} \mathbf{V}$ can be implemented with dense matrix algebra and the remaining calculations, that involve only matrix multiplications, can be implemented with matrix-free techniques. The downside of this approach, as opposed to the reduced-rank pseudo-inverse above, is that the result is dependent on the structure of the prior covariance matrix. Therefore, while this may benefit the sparsity to speedup the calculations, the arbitrariness in the prior selection enters the solution and it seems impossible to separate the pure Hessian contribution.

Rewriting (2.7.6) allows matrix-free implementation for large prior matrices that are available only through matrix-vector products

$$
\begin{aligned}
& \mathbf{P}=\left[\mathbf{I}-\mathbf{P}_{0} \mathbf{V}\left(\boldsymbol{\Lambda}^{-1}+\mathbf{V}^{T} \mathbf{P}_{0} \mathbf{V}\right)^{-1} \mathbf{V}^{T}\right] \mathbf{P}_{0}=\left[\mathbf{P}_{0}\left[\mathbf{I}-\mathbf{P}_{0} \mathbf{V}\left(\boldsymbol{\Lambda}^{-1}+\mathbf{V}^{T} \mathbf{P}_{0} \mathbf{V}\right)^{-1} \mathbf{V}^{T}\right]^{T}\right]^{T} \\
& \mathbf{P}=\mathbf{P}_{0}\left[\mathbf{I}-\mathbf{V}\left(\boldsymbol{\Lambda}^{-1}+\mathbf{V}^{T} \mathbf{P}_{0} \mathbf{V}\right)^{-1} \mathbf{V}^{T} \mathbf{P}_{0}\right]=\mathbf{P}_{0}\left[\mathbf{I}-\left[\mathbf{P}_{0} \mathbf{V}\left(\boldsymbol{\Lambda}^{-1}+\mathbf{V}^{T} \mathbf{P}_{0} \mathbf{V}\right)^{-1} \mathbf{V}^{T}\right]^{T}\right]
\end{aligned}
$$

The last right hand side transformations in both formulae are strictly correct only for symmetric prior matrices, which is always the case for covariances. A convenient interpretation is obtained by rearranging

$$
\mathbf{P} \mathbf{P}_{0}^{-1}=\mathbf{I}-\mathbf{P}_{0} \mathbf{V}\left(\boldsymbol{\Lambda}^{-1}+\mathbf{V}^{T} \mathbf{P}_{0} \mathbf{V}\right)^{-1} \mathbf{V}^{T}
$$

with the right hand side of (2.7.10) seen as an uncertainty reduction operator. For diagonal $\mathbf{P}_{0}$ the diagonal of (2.7.10) is the rate of uncertainty variance reduction. Another 
measure of uncertainty reduction, valid for any $\mathbf{P}_{0}$, is direct comparison of prior and posterior diagonals:

$$
1-\sqrt{\frac{\operatorname{diag}(\mathbf{P})_{i}}{\operatorname{diag}\left(\mathbf{P}_{0}\right)_{i}}}
$$

which is the same as the posterior relative error reduction in Losch and Wunsch (2003).

Alternative development (Y. Marzouk, Large-scale Inverse Problems and Quantification of Uncertainty workshop, IMA 2011) is possible by simultaneous diagonalization of the Hessian and of the prior matrix, which eliminates the need for numerical matrix inversion at the expense of generalized eigenvalue decomposition and loss of strict orthogonality of the eigenvectors. The inversion then is achieved with the analytical formula and the resulting generalized eigenvectors are prior-orthogonal, i.e. under prior-weighted inner product. The significant advantage of that approach is due to the closed form expression of the posterior covariances which can be analyzed analytically. The disadvantage is in the dependence on the arbitrary prior choice, which becomes an inherent part of the solution.

For approximately singular Hessian it is also possible to construct a rank-1 vector update scheme to iteratively update the solution up to sufficient convergence. This is not pursued in this thesis.

\subsection{Forward uncertainty propagation}

In the developed UQ framework two options are possible for forward uncertainty propagation. One can choose to restrict the analysis only to the sub-domain supported by the observations, where the "posterior" uncertainty covariance is given by (2.7.2). The forward uncertainty propagation is then given by

$$
\mathbf{T} \mathbf{H}^{+} \mathbf{T}^{T}=\mathbf{T V} \boldsymbol{\Lambda}^{-1} \mathbf{V}^{T} \mathbf{T}^{T}=\sum_{i} \frac{1}{\lambda_{i}} \mathbf{T} \mathbf{v}_{i} \mathbf{v}_{i}^{T} \mathbf{T}^{T}
$$

which can be implemented as a sum of rank-1 matrix updates. Here we shorten the notation by defining the target transformation $\mathbf{T}$ equal to the Jacobian matrix $\partial N / \partial \mathbf{x}^{T}$. Note, that this result does not represent the formal error covariance of the target variable 
when the data-supported sub-domain of controls, while excluding the nullspace of the observation operator, also excludes components of the target transformation row space. That is, if the excluded combinations of control variables which are not constrained by the observations, are not also in the nullspace of the target transformation and thus map their infinite uncertainty to the target variable range.

Alternatively, for the regularized posterior uncertainty of controls (2.7.6) the posterior target uncertainty is calculated by

$$
\mathbf{T P} \mathbf{T}^{T}=\mathbf{T P}_{0} \mathbf{T}^{T}-\mathbf{T} \mathbf{P}_{0} \mathbf{V}\left(\boldsymbol{\Lambda}^{-1}+\mathbf{V}^{T} \mathbf{P}_{0} \mathbf{V}\right)^{-1} \mathbf{V}^{T} \mathbf{P}_{0} \mathbf{T}^{T}
$$

or with a matrix-free implementation by

$$
\mathbf{T P T}^{T}=\mathbf{T P}_{0} \mathbf{T}^{T}-\mathbf{T} \mathbf{P}_{0} \mathbf{V}\left[\mathbf{T P}_{0} \mathbf{V}\left(\boldsymbol{\Lambda}^{-1}+\mathbf{V}^{T} \mathbf{P}_{0} \mathbf{V}\right)^{-T}\right]^{T}
$$

Remember, that the prior target uncertainty is given by $\mathbf{T P}_{0} \mathbf{T}^{T}$ and only the second right hand side term needs to be calculated for target uncertainty reduction. If the target model is not linear but (2.3.10) applies, the Jacobian derivative code needs to be implemented. For a single scalar target variable (2.3.12) the implementation is straightforward with the standard adjoint code of the target model, as the target transformation $\mathbf{T}$ is replaced by the single row vector $\partial N / \partial \mathbf{x}^{T}$, i.e. the transposed gradient of the target variable.

For a multivariate target variable vector either a full Jacobian matrix code is required or a Jacobian-vector or a vector-Jacobian products codes, depending on the relative dimensionality of the target and the control spaces. In particular a vector adjoint code can be generated in scalar TAF mode to efficiently evaluate the reverse mode vector-Jacobian products if the number of target variables is much smaller that the dimensionality of the control space. 


\subsection{Summary of proposed UQ schemes}

For quick reference, we summarize the developed UQ algorithms. The workflow of the proposed inverse-predictive uncertainty propagation procedures, which formalize (2.1.6), is illustrated below (2.9.1). Observation data uncertainty is assimilated into the model by projection onto model controls by the inverse uncertainty propagation procedure (2.3.4). Then, the assimilated uncertainty is projected forward onto the desired target variables (2.3.10), here for simplicity of presentation shown for a single target scalar $z$.

$$
\begin{aligned}
& \text { Data uncertainty } \rightarrow \text { Controls uncertainty } \rightarrow \text { Target uncertainty } \\
& \begin{array}{llll}
\mathbf{P}_{\mathrm{yy}} & \rightarrow \quad \mathbf{P}_{\mathrm{xx}} & \rightarrow & \sigma_{z}^{2}
\end{array}
\end{aligned}
$$

The implementation of this scheme is straightforward for invertible models by inversion of the Hessian of the non-regularized data-model misfit function $J_{1}$ (2.2.1) and its forward projection, here given by the target gradient vector $\mathbf{g}$ (2.9.2). This uncertainty assimilation procedure is independent of a priori assumed uncertainty of controls and thus can be regarded as "pure" propagation of observations' uncertainty (2.9.2). Color coding is introduced in schematics below, intended to help differentiate between the three different uncertainties of controls recurring in this thesis: the "pure" prior-independent assimilated uncertainty $\mathbf{P}_{\mathbf{x x}}$ (green), the assumed prior uncertainty $\mathbf{P}_{0}$ (blue) and the posterior assimilated uncertainty $\mathbf{P}$ (black). The uncertainty of observations is shown in red given by the covariance matrix $\mathbf{P}_{\mathbf{y y}}=\mathbf{R}$. This color coding is utilized in UQ illustration figures in Section 2.10.

$$
\begin{array}{llll}
\mathbf{P}_{\mathrm{yy}} \longrightarrow \mathbf{P}_{\mathrm{xx}}=\left(\mathbf{H}_{J_{1}}\right)^{-1} \longrightarrow \sigma_{z}^{2}=\mathbf{g}^{T} \mathbf{P}_{\mathrm{xx}} \mathbf{g} \\
& & \\
& \mathbf{P}_{0} & & \\
& \downarrow & & \\
& & & \\
& & & \\
\mathbf{P}_{\mathrm{yy}} \longrightarrow \sigma_{z}^{2}=\mathbf{g}^{T} \mathbf{P}_{0} \mathbf{g}
\end{array}
$$

For the ill-posed inverse problems in oceanographic applications such prior-independent uncertainty assimilation schemes (2.9.2) lead to unbounded uncertainties because not all model controls are constrained by the assimilated uncertainty of observations. In these 
cases we can resort to the prior-to-posterior uncertainty reduction scheme (2.9.3), which melds together the assimilated observations uncertainty with prior uncertainty of the controls to produce the posterior controls uncertainty. The melding can be computed by inverting the Hessian of the regularized data-model misfit function $J$ (2.2.6) or with the reduced order method based on (2.7.6). Both the prior and the posterior uncertainties of controls can be forward projected onto the target variable space, where both can be compared, which is guaranteed to result in prior-to-posterior target uncertainty reduction.

\subsection{Synthetic UQ problem and the "identical twins" setup}

The initial application of the proposed estimation and UQ methodology is implemented in a simplified synthetic framework also known as the "identical twins experiment" (Losch and Wunsch 2003). The synthetic ocean state estimation problem is designed by first selecting a value, $\tilde{\mathbf{x}}$, for the estimated parameter $\hat{\mathbf{x}}=\tilde{\mathbf{x}}$ and applying the forward model to find its image $\tilde{\mathbf{y}}=M(\tilde{\mathbf{x}})$. To focus the attention on quantification of the uncertainty itself and to avoid implementation of minimization algorithm, we consider the simplest inverse problem case by choosing the prior mean $\mathbf{x}_{0}=\tilde{\mathbf{x}}$ and observation $\mathbf{y}=\tilde{\mathbf{y}}$. This leads to an unbiased and zero residual inverse problem, allowing arbitrary specification of the prior and the observation error covariance matrices. The Hessian of model-data misfits needs to be evaluated at the solution point $\tilde{\mathbf{x}}$, and the nonlinear Hessian term disappears because the residual misfit is zero by construction.

In our MITgcm implementation, the estimated parameters are the perturbations of ocean model control fields. We construct the synthetic inverse problem by setting the perturbations to be zero. Consequently, the Hessian model is executed unperturbed.

The synthetic decoupling between the calculations of nonlinear state estimation and its UQ is useful for optimal design of observation systems, extending similar decoupling practice in linear estimation problems. For a linear inverse problem, its Hessian and therefore UQ calculation are independent of the data, allowing "precomputing" the uncertainty even before the data is collected. Similarly, the " identical twins" setup allows data independent UQ analysis for nonlinear inverse problems. One can use the 
uncertainty estimates from the "identical twins" calculation for design of optimal observation systems and then after the data is acquired and the nonlinear inverse problem actually solved - another UQ calculation based on the actual (not synthetic) estimated controls can confirm the "identical twins" estimates or quantify any corrections if needed.

\subsection{Elementary illustration in two dimensions}

We illustrate the developed uncertainty assimilation machinery in the "identical twins experiment" setup with a simple analytical example in two dimensions. Consider a linear forward model given by a regular invertible linear transformation

$$
\mathbf{M}_{1}=\left[\begin{array}{cc}
0 & 0.4 \\
-0.4 & 0
\end{array}\right]
$$

The forward model maps controls $\mathbf{x}$ to observations $\mathbf{y}$, both shown as 2 -d vectors in Figure 2.1. Uncertainty covariances are shown by the corresponding constant- $\sigma$ ellipses, i.e. $\mathbf{x}^{T} \mathbf{C}^{-1} \mathbf{x}=\sigma^{2}$.

The uncertainty of observations ${ }^{10}$ (red) is mapped back by (2.3.4) to the model controls (green), which is defined as assimilated uncertainty. Note, that since the forward model is invertible, the assimilated uncertainty of controls can be forward projected back to the observations space by (2.3.3). Such one-to-one back and forth transformations would complete the uncertainty propagation operations for any invertible forward model. However, for non-invertible models such one-to-one inverse uncertainty propagation is not possible. The alternatives are either to explicitly analyze the range and the nullspace of their Hessian or to calculate the prior-to-posterior reduction of uncertainty. For completeness of the illustration Figure 2.1 visualizes the later for the invertible model case.

To illustrate the UQ alternative for a more general non-invertible model, consider a singular linear transformation in 2-d given by the projection on vector $\mathbf{u}=\left[\begin{array}{ll}0.2 & -0.3\end{array}\right]^{T}$

$$
\mathbf{M}_{2}=\mathbf{u}\left(\mathbf{u}^{T} \mathbf{u}\right)^{-1} \mathbf{u}^{T} \approx\left[\begin{array}{cc}
0.31 & -0.46 \\
-0.46 & 0.69
\end{array}\right]
$$

10 The uncertainty of observations, the assimilated uncertainty, the prior and posterior uncertainties of the solution are given by the covariance matrices

$\mathbf{P}_{\mathbf{y y}}=\left[\begin{array}{cc}63 & 47 \\ 47 & 253\end{array}\right] \times 10^{-4}, \quad \mathbf{P}_{\mathbf{x x}}=\left[\begin{array}{cc}158 & -29.7 \\ -29.7 & 39\end{array}\right] \times 10^{-3}, \quad \mathbf{P}_{0}=\left[\begin{array}{cc}90 & 40 \\ 40 & 150\end{array}\right] \times 10^{-3}, \quad \mathbf{P}=\left[\begin{array}{cc}50 & -3 \\ -3 & 27\end{array}\right] \times 10^{-3}$ 


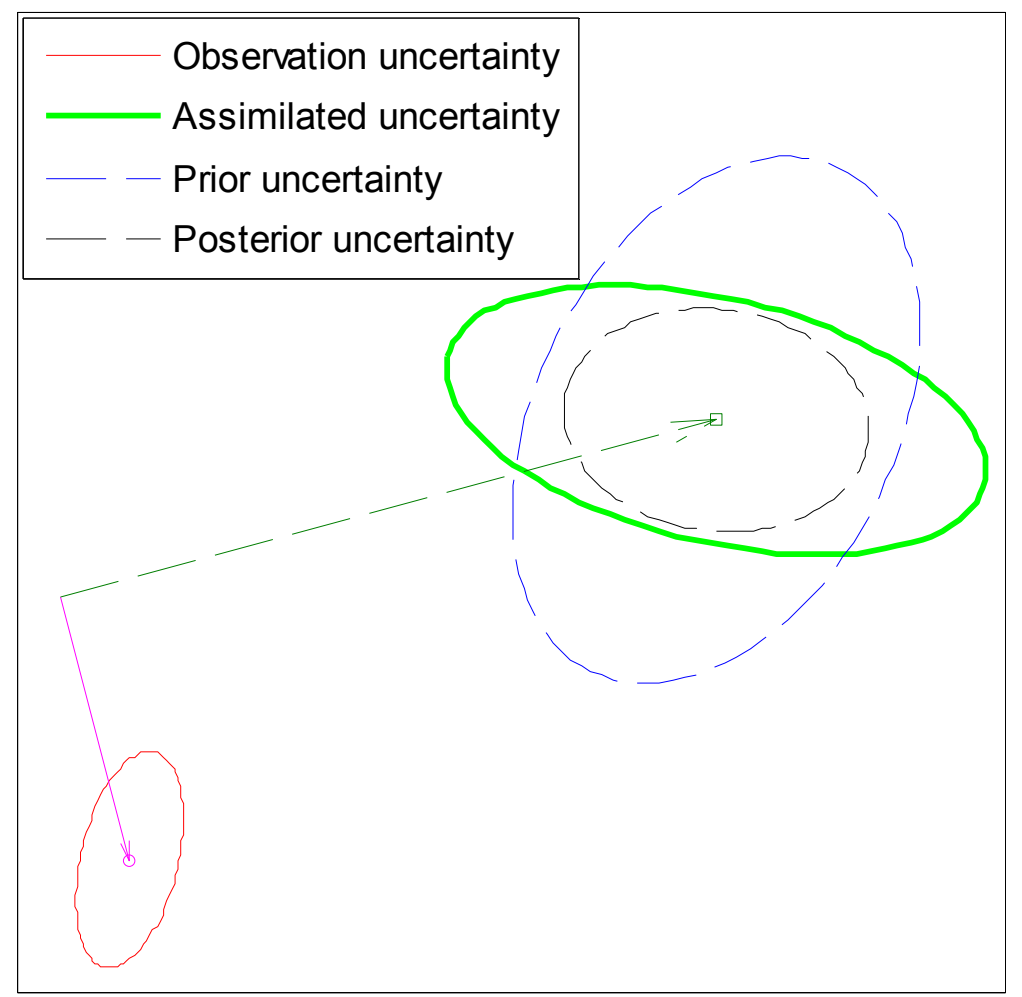

Figure 2.1 Visualization of inverse and posterior uncertainty problem for linear transformation $\mathrm{M}_{1}$. Ellipses are shown for $\sigma$ value 1 .

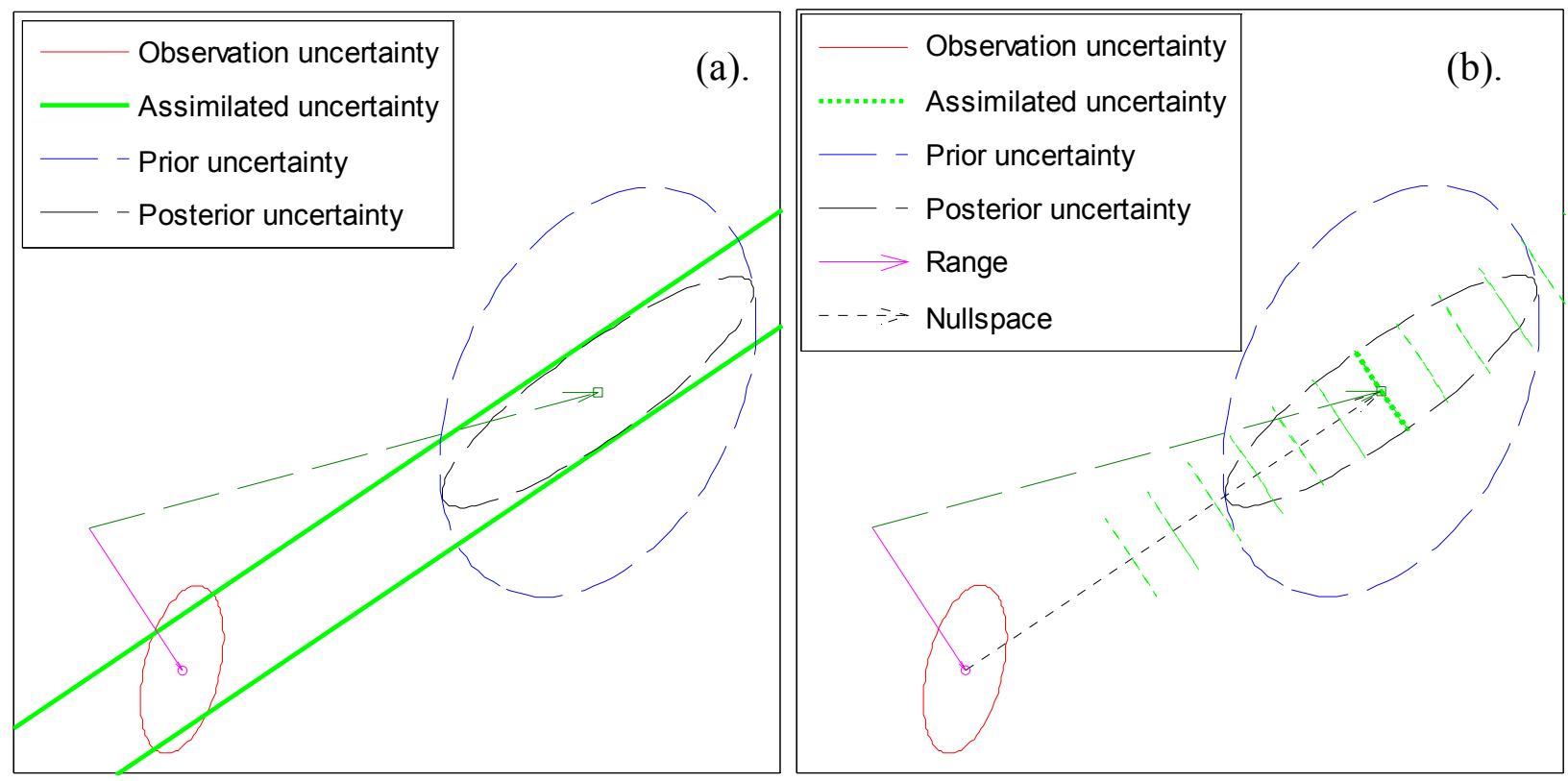

Figure 2.2 Visualization of inverse and posterior uncertainty problem for singular linear transformation $\mathrm{M}_{2}$. The uncertainty of observations and the prior uncertainty are the same as in the previous invertible case.

Direct application of the uncertainty inversion transformation (2.3.4), as in the case of invertible models, results in assimilated uncertainty which is ill defined. In fact, the exact 
inversion calculation fails due to matrix singularity and no uncertainty ellipse is available for visualization. Nonetheless, these circumstances can be understood by the illustrating the inversion in the limit of a nearly singular matrix, by plotting the inversion with a slightly perturbed forward model given by the finite precision approximation (2.11.2). The outcome of inverting a non-invertible model is infinite uncertainty in certain directions which are not constrained by the finite uncertainty of the observations. This infinite uncertainty is illustrated by the infinite elongated ellipse of the assimilated uncertainty in Figure 2.2(a). Despite the difficulty with direct inversion, the calculation of the prior-to-posterior uncertainty reduction (2.7.6) always succeeds. The plotted posterior uncertainty ellipse highlights graphically the lack of uncertainty reduction in the unconstrained direction, while the reduction in the constrained direction is exactly consistent with the direct inversion in the near singular asymptotic limit.

The alternative to the prior-to-posterior calculation is the detailed analysis of the range and the nullspace of the Hessian, which in this simple case are identical to the range and the nullspace of the forward model, whose dimension here is one. The nullspace vector and the range vector are shown in Figure 2.2(b). The nullspace is the direction along which the controls are projected onto the observations space, spanned by the range vector. Because the projection strips out all of the information along the nullspace direction, its inverse cannot reconstruct it and this direction is not constrained by the assimilation of the observation uncertainty. The information along the range direction is preserved by the projection and therefore the inversion of the observation uncertainty constrains the uncertainty of the controls in that direction. Although one cannot plot an ellipse of the assimilated uncertainty in whole control space, one can plot it in the constrained sub-space. The position of this sub-space ellipse in the full control space is not known, as it can be arbitrarily shifted along the unconstrained directions of the nullspace. This is explicitly visualized in Figure 2.2(b). by the 1-dimensional collapsed ellipses of the assimilated uncertainty. The length of the remaining uncollapsed semi-axis is equal the square root of the inverse of the non-zero eigenvalues of the Hessian. The direction of the semi-axis is the corresponding eigenvector of the Hessian. In general, the full structure of the finite covariance in the reduced data-supported sub-space is given by the pseudo-inverse of the Hessian (2.7.2). 


\subsection{Formalism for uncertainty quantification in ocean state estimation}

Although the developed algebraic formalism is general enough and allows implementation for ocean state estimation by defining all the dependent variables as controls, here we explicitly rewrite the UQ problem formulation in the original control theoretic formalism of ocean state estimation problem (Wunsch and Heimbach 2007). Following Wunsch and Heimbach, the equations of a nonlinear ocean model can be written

$$
x_{t+\Delta t}=\mathcal{L}\left(x_{t}, q_{t}, u_{t}, t\right)
$$

where $x_{t}$ is the instantaneous model state vector, $q_{t}$ is the deterministic forcing vector and $u_{t}$ is the unknown control vector. Note, the nonlinear model is explicitly time dependent (non-autonomous, not time invariant). Let the instantaneous vector of model diagnostics or model counterparts of the observed data be given by a nonlinear equation

$$
y_{t}=F\left(x_{t}, t\right)+n_{t}
$$

where $n_{t}$ represents random observation noise. Ultimately, the objective of ocean state estimation and uncertainty quantification is a target variable derived form the state of the ocean by the nonlinear equation

$$
z_{t}=\mathcal{T}\left(x_{t}, t\right)
$$

Here we did not include a random noise or model error term, although this can be straightforwardly added to the formulation.

To simplify connection to the $\mathrm{AD}$ formulation and for consistency with control theoretical formalism, we can write the solution of the ocean model as integrated trajectory in model state space

$$
\begin{gathered}
\mathbf{x}=\mathbf{M}\left(x_{0}, \mathbf{u}\right) \\
\mathbf{y}=\mathbf{F}(\mathbf{x})+\mathbf{n} \\
\mathbf{z}=\mathbf{T}(\mathbf{x})
\end{gathered}
$$

The vectors shown in bold are trajectories of the corresponding instantaneous vectors model state, controls, modeled observations, observation noise and target variable. We note that this formalism explicitly separates the dependence of the model trajectory on the initial conditions from the vector of model controls, in contrast to (2.1.1). 


\section{Barotropic models of ACC and flow through the Drake Passage}

\subsection{Analytical ODE model}

Consider the barotropic physics of a wind driven circumpolar current over varying bottom topography with parameterized bottom friction. The nonlinear barotropic equations with surface wind stress $\tau_{s}$ and linear bottom drag coefficient $r$ are

$$
\begin{gathered}
\frac{\partial u}{\partial t}+u u_{x}+v u_{y}=f v-g \eta_{x}+\frac{\tau_{s}^{x}}{\rho H}-\frac{r u}{H} \\
\frac{\partial v}{\partial t}+u v_{x}+v v_{y}=-f u-g \eta_{y}+\frac{\tau_{s}^{y}}{\rho H}-\frac{r v}{H} \\
\frac{\partial \eta}{\partial t}=-(u(H+\eta))_{x}-(v(H+\eta))_{y}
\end{gathered}
$$

Linearized equations reduce to

$$
\begin{gathered}
\frac{\partial u}{\partial t}=f v-g \eta_{x}+\frac{\tau_{s}^{x}}{\rho H}-\frac{r u}{H} \\
\frac{\partial v}{\partial t}=-f u-g \eta_{y}+\frac{\tau_{s}^{y}}{\rho H}-\frac{r v}{H} \\
\frac{\partial \eta}{\partial t}=-(u H)_{x}-(v H)_{y}
\end{gathered}
$$

To capture the leading order dynamics we derive a simplified model of ACC adjustment to steady state forced by zonal wind stress only. The steady state balance equations reduce to

$$
\begin{gathered}
0=f v-g \eta_{x}+\frac{\tau_{s}^{x}}{\rho H}-\frac{r u}{H} \\
0=-f u-g \eta_{y}-\frac{r v}{H} \\
0=(u H)_{x}+(v H)_{y}
\end{gathered}
$$

For a flat bottom ocean the continuity equation (3.1.6) reduces to $0=u_{x}+v_{y}$.

If zonal homogeneity is assumed $(\partial / \partial x=0)$, integration of the flat bottom continuity equation from the southern boundary imposes zero meridional flow all over the domain and the steady state solution simplifies to 


$$
\begin{aligned}
& 0=\frac{\tau_{s}^{x}}{\rho H}-\frac{r u}{H} \\
& 0=-f u-g \eta_{y}
\end{aligned}
$$

The zonal momentum equation has reduced to a simple balance between wind forcing and bottom friction, while the meridional equation simplified to the geostrophic balance accompanying the zonal flow. The transient adjustment to this steady state can be described in bulk for a zonally periodic basin when meridional flow perturbations can be neglected in the momentum equations by

$$
\frac{\partial u}{\partial t}=\frac{\tau_{s}^{x}}{\rho H}-\frac{r u}{H} .
$$

This idealized form of zonal momentum balance in a zonally homogeneous flat bottom case can be written as a simple linear ODE:

$$
\frac{d u}{d t}=-\frac{r}{H} u+\frac{\tau_{s}^{x} / \rho}{H} \equiv-R u+F
$$

Here $R$ and $F$ are just depth-normalized constant dissipation and forcing parameters. The general solution is of the form of exponential relaxation, given by

$$
u(t)=\left(u_{0}-\frac{F}{R}\right) e^{-R\left(t-t_{0}\right)}+\frac{F}{R}=u_{0} e^{-\frac{r}{H}\left(t-t_{0}\right)}+\frac{\tau_{s}^{x} / \rho}{r}\left(1-e^{-\frac{r}{H}\left(t-t_{0}\right)}\right)
$$

The system adjusts to a steady state independent of the initial conditions $u_{0}$ with the relaxation time scale $H / r=1 / R$. This time scale is also the exponential rate of decay of the transient effect of the initial conditions. The asymptotic steady state flow is the balance of wind stress and bottom drag, independent of the water column depth:

$$
u_{\infty}=\frac{F}{R}=\frac{\tau_{s}^{x} / \rho}{r}
$$

Similar idealized solution was utilized in previous theoretical studies of barotropic ACC dynamics (Wearn and Baker 1980, Weijer and Gille 2005, Olbers and Lettmann 2007). We highlight that this analytical model is an oversimplification of the real dynamics, it does not resolve the detailed spatial structure of the geostrophic adjustment process and is intended to describe the integral balances in a bulk form. The following detailed numerical simulations with a nonlinear barotropic model confirm the high degree of accuracy that this simple ODE model describes the overall system dynamics. 


\subsection{Barotropic scales of ACC transport}

First we consider the characteristic magnitudes of the physical and geometric variables involved in the observed Drake Passage transport. These scales will guide our theoretical understanding of the dynamics governing the Drake Passage transport and will be used for design of equivalently scaled idealized simulations.

Despite the uncertainty and the variability debated in the literature, the rate of Drake Passage volume transport is estimated to $O(100 \mathrm{~Sv})$, i.e. $10^{8} \mathrm{~m}^{3} / \mathrm{s}$. Examples include the early observational estimate of $110 \mathrm{~Sv}$ (Meredith et al 2011), and the high resolution ocean state estimation system result $153 \mathrm{~Sv}$ (Mazloff et al. 2010). For the characteristic ocean depth of $5 \mathrm{~km}$ and the rough scale of the meridional extent of the Drake Passage of 10 degrees or about $1.1 \times 10^{3} \mathrm{~km}$, the cross-sectional area of the passage is $5.5 \times 10^{9} \mathrm{~m}^{2}$. Thus, the typical average zonal flux $U$ is order of $2 \mathrm{~cm} / \mathrm{s}$, which is in good agreement with the scale of measured horizontal velocities at the Passage (Meredith et al 2011). The corresponding scale of geostrophic surface elevation slope is $\eta_{y}=-f U / g \approx 2 \times 10^{-7}$, translating to surface elevation difference across the Passage of $0.2 \mathrm{~m}$.

If simple barotropic balance of wind stress and bottom drag (3.1.7) is assumed, the scales of wind stress and friction coefficient are constrained by the scale of the barotropic flow rate $U=2 \mathrm{~cm} / \mathrm{s}$. Following Losch and Wunsch (2003), the scale of the linear bottom drag coefficient is assumed $r=5 \times 10^{-3} \mathrm{~m} / \mathrm{s}$, which for $5000 \mathrm{~m}$ deep water column translates to spin down time scale $H / r=10^{6}$ s or about 12 days. The corresponding kinematic wind stress scale is $\tau_{s}^{x} / \rho=r U=10^{-4} \mathrm{~m}^{2} \mathrm{~s}^{-2}$, i.e. wind stress $\tau_{s}^{x}=10^{-1} \mathrm{~Pa}$.

The Rossby number for Drake transport related barotropic flow dynamics can be estimated with the length scale given by the meridional extent of the Drake Passage $\left(L=10^{6} \mathrm{~m}\right)$ and the velocity scale given by the barotropic flow rate $\left(U=2 \times 10^{-2} \mathrm{~m} / \mathrm{s}\right)$. The resulting Rossby number is $\operatorname{Ro}=U(L f)^{-1}=2 \times 10^{-4}$, which is sufficiently small to expect a negligible contribution from the nonlinear advection terms in the momentum equations. The real turbulent baroclinic ACC dynamics is more nonlinear than this, but we restrict our simplified analysis to the coarse barotropic scales.

The time dependent solution (3.1.10) of the proposed simplified analytical ODE model of the circumpolar transport is governed by a single time scale, given by the frictional spin- 
down rate. This frictional time scale is inversely proportional to the bottom friction coefficient and is linear in the depth of the ocean:

$$
T_{\text {friction }}=\frac{H}{r}
$$

Therefore, in deeper basins or when the friction is weaker, the dynamics of ACC evolution is, to the leading order, controlled by a single slower time scale. However, in detailed numerical PDE model solutions that extend to the equator and include continents, additional time scales (slower or faster) are expected to influence the circumpolar transport evolution, reflecting the dynamics of different barotropic waves (see Figures 3.1 and 3.2). Fast Kelvin waves propagating along the coastal boundaries and the equatorial wave guide with phase speed $\sqrt{g H}=220 \mathrm{~m} / \mathrm{s}$ traverse a length comparable to the Drake Passage in $O(1$ hour $)$ and cross the Pacific ocean in $O(1$ day).
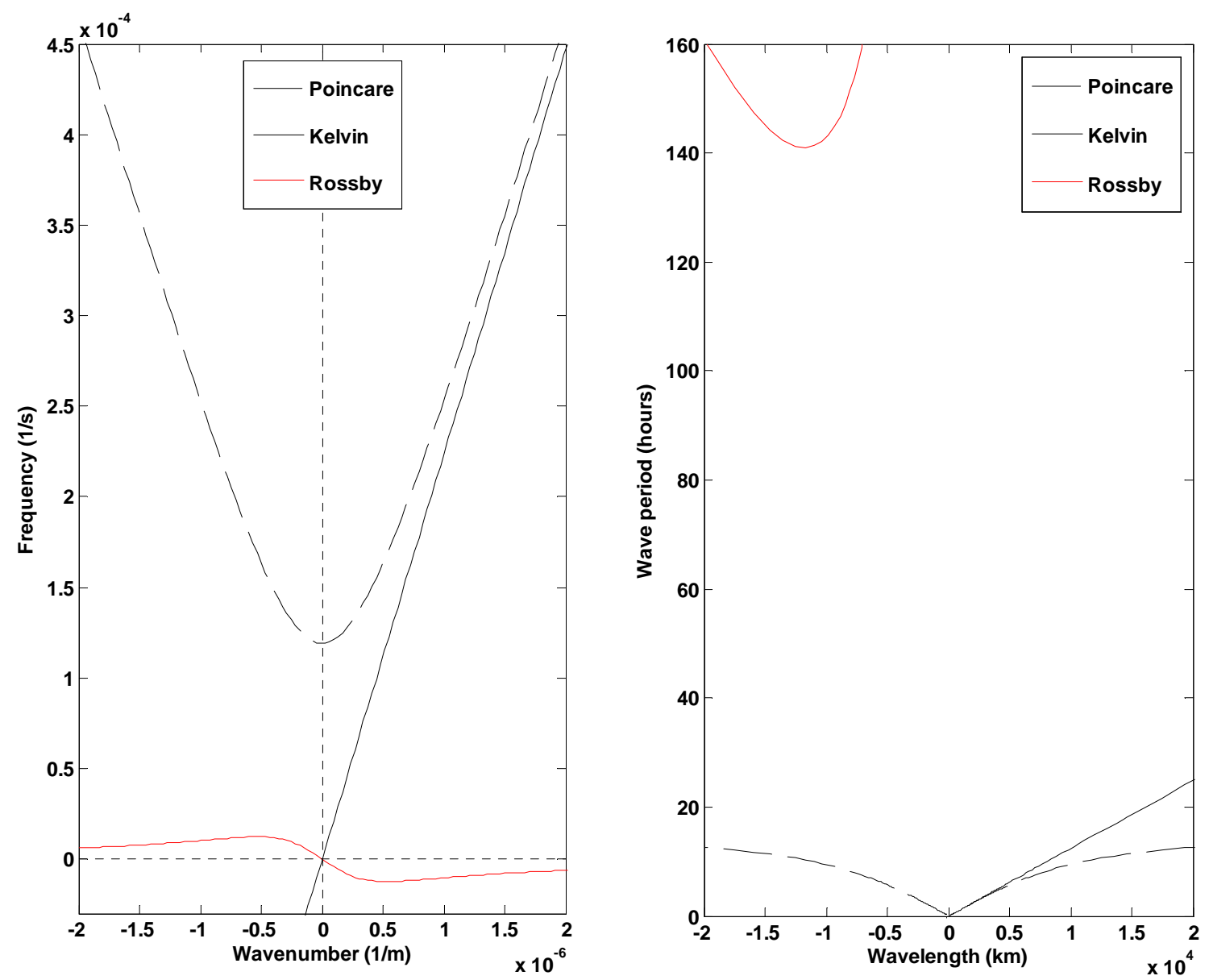

Figure 3.1 Dispersion relations (left) and characteristic scales (right) of barotropic waves at the latitude of the Drake Passage (shown for latitude $-55^{\circ}$ ). The theory of midlatitude barotropic waves is a standard textbook material, see for example Chapter 9 of Cushman-Roisin and Beckers (2011). 
Faster inertia-gravity (Poincare) waves can cross the Pacific ocean in few hours or less, propagating geostrophic adjustment disturbances with effectively unbounded phase speeds. They oscillate on the inertial time scale $O(10$ hours $)$ and below, asymptoting to the Kelvin wave periods for shorter wavelengths. Slower planetary (Rossby) waves oscillate at Drake Passage latitudes with periods longer than 6 days. Long $O\left(10^{4} \mathrm{~km}\right)$ planetary disturbances propagate non-dispersively at $45 \mathrm{~m} / \mathrm{s}$ crossing the Pacific from east to west in 5 days. Equatorial Rossby waves (Figure 3.2) are about a factor of 2 faster, with peak non-dispersive propagation speed of $74 \mathrm{~m} / \mathrm{s}$ and shortest oscillation period of 3.5 days. If the asymmetric mixed equatorial mode $(n=0)$ is excited on the large scale it can propagate across the Pacific arbitrarily fast.
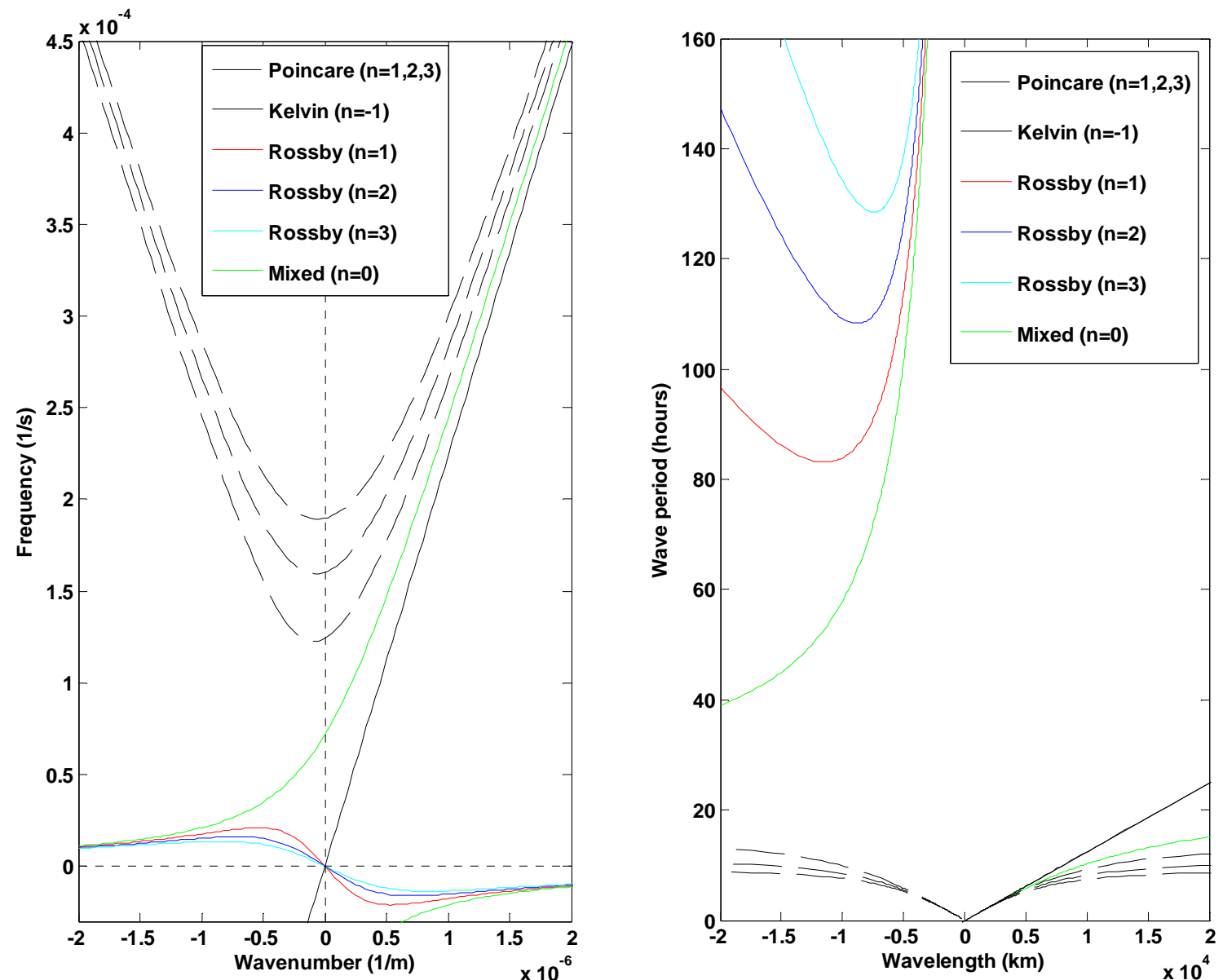

Figure 3.2 Dispersion relations (left) and characteristic scales (right) of equatorial barotropic waves shown for different modes $n$, representing different meridional profiles spanned by Hermite polynomials $H_{n}(y)$. Further details of equatorial barotropic waves can be found in Cushman-Roisin and Beckers (2011) Chapter 21.

In addition to resolving the barotropic dynamics, the numerical implementation of the PDE model introduces another time scale. The model is stabilized with numerical 
dissipation based on the Smagorinsky viscosity scale. For lateral eddy viscosity coefficient $\mathrm{A}_{h}=4 \times 10^{2} \mathrm{~m}^{2} / \mathrm{s}$ the horizontal momentum diffusion introduces a long spin down time scale $T_{\mathrm{A}_{h}}=0.25 \cdot \Delta x^{2} / \mathrm{A}_{h}$, reaching for a $200 \mathrm{~km}$ spatial grid resolution $O$ (300 days).

\subsection{Numerical PDE models}

While the idealized analytical ODE model is a useful simplification of the ACC dynamics, it does not solve the primitive ocean equations and does not resolve the multitude of degrees of freedom of the actual ocean physics. The resolution of the multivariate spatio-temporal dynamics of ocean fields requires a PDE model. We use the MITgcm modeling system (Marshall et al. 1997a,b, http://mitgcm.org/) to simulate the global barotropic dynamics of ACC in several idealized and realistic configurations of increasing complexity and detail. We pursue this hierarchical modeling strategy because, while the realistic configuration is the closest to describing the actual observed physics, it masks the detailed understanding of the dynamics by the many layers of its complexity. The idealized configurations simplify the resolved dynamics while attempting to capture only the essential mechanisms in a hierarchy of configuration geometries.

The barotropic dynamics of ACC are modeled in a single layer global spherical domain configuration. The spatial resolution is set to 2 by 2 degrees with zonally periodic domain extending from latitude 80 south to 80 north. In the idealized flat bottom configurations the water depth is set to $5000 \mathrm{~m}$. We follow Losch and Wunsch (2003) setting the linear bottom drag coefficient to $5 \times 10^{-3} \mathrm{~m} / \mathrm{s}$. Homogeneous steady zonal wind forcing is specified by a sinusoidal profile, as detailed in the figures below. Other numerical MITgcm parameters are listed in Appendix B.

\subsubsection{Idealized geometry - aqua planet}

The simplest idealized global PDE model of ACC is constructed on a spherical aqua planet with flat bottom and forced by a constant zonally homogeneous zonal wind stress. MITgcm solves the nonlinear barotropic equations starting from zero initial conditions and converging to a steady state after 100 days. The convergence is confirmed by running the model for 1 and 10 years. The steady state equilibrium exhibits exact balance of wind 
forcing with bottom drag in accordance with our ODE model (3.1.9). The modeled idealized ACC jet is aligned with the applied wind stress (Figure 3.3), flow velocity is equal the forcing to friction ratio given by (3.1.11). The flow streamlines and surface elevation contours are aligned in geostrophic balance creating the typical ACC frontal structure and supporting our theoretical analysis (3.1.7). The meridionally integrated zonal transport at the steady state is $112 \mathrm{~Sv}$.

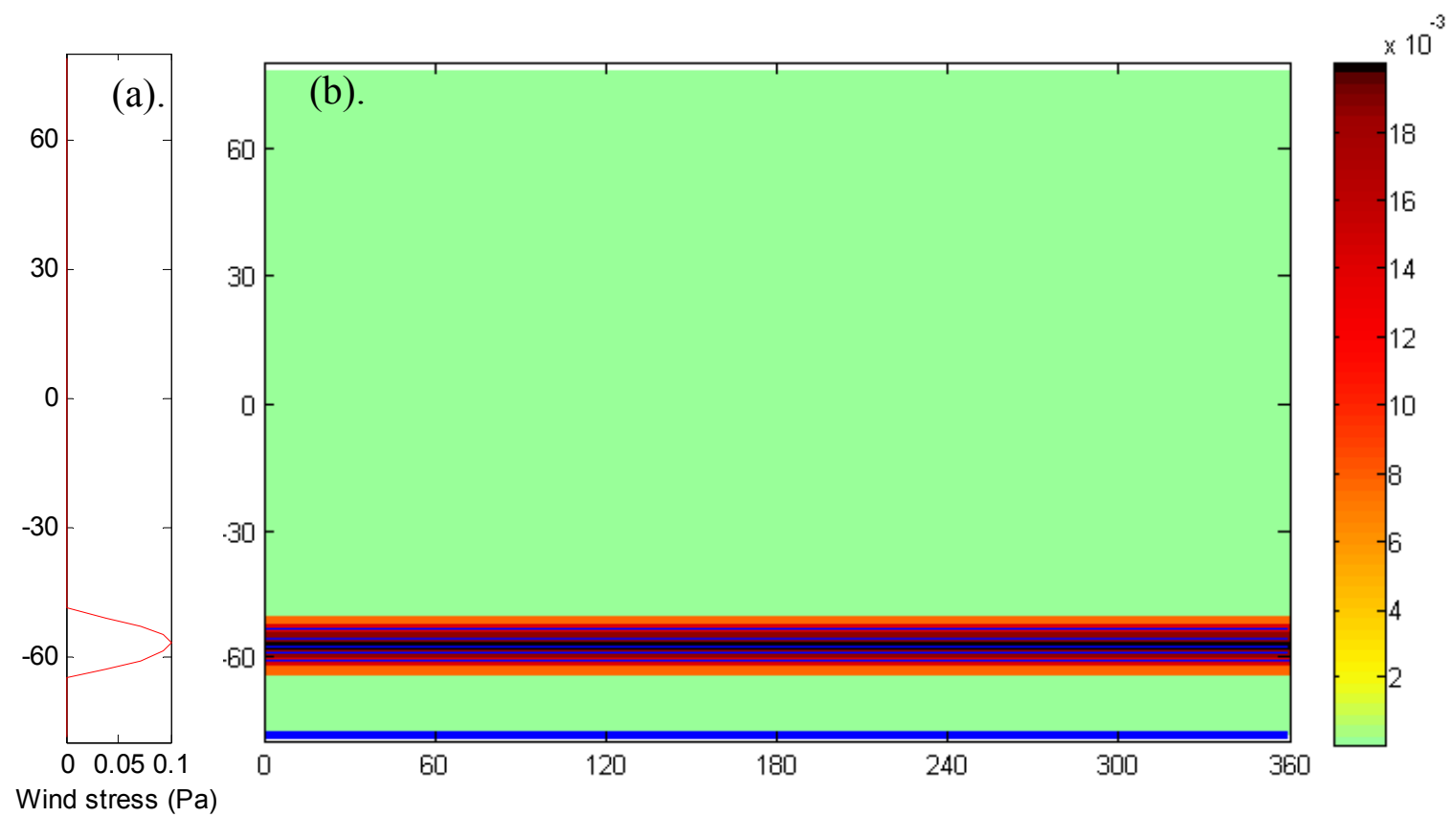

Figure 3.3 Wind stress profile (a) and barotropic flow map (b) shown by the magnitude of 2-D velocity vector (color shades) overlaid with sea surface height contours (blue).

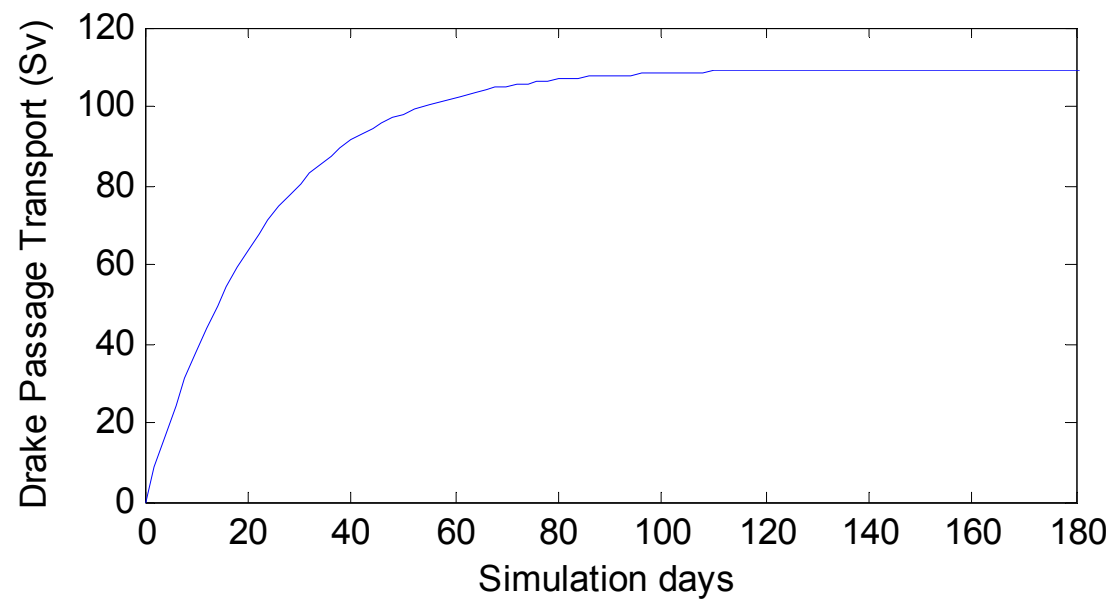

Figure 3.4 Drake Passage transport growth to steady state.

Figures 3.5 and 3.6 display a detailed analysis of the geostrophic adjustment dynamics. Starting from rest the flow accelerates eastward under the forcing of the wind, but outside 
of the latitudes of the applied wind forcing a return flow develops (Figure 3.5). This eastto-west return flow is only a transient feature, as at the steady state there is no flow outside of the wind forced region. The transient zonal return flow is accompanied by a transient northward transport, which can be explained by the geostrophic adjustment to the applied wind forcing. This transient northward transport redistributes the water mass across the wind jet to create the meridional surface elevation gradient. That northward sea surface gradient creates a southward pressure gradient force beneath it, which in the steady state balances the northward (equatorward) Coriolis force on the zonal geostrophic jet. The transient northward transport is also subjected to the Coriolis force, which pushes the flow westward, explaining the return flow.

More details are available by examining the balance of the forces (Figure 3.6). The main balance in the zonal direction is between the wind stress and the bottom friction, which fully balance each other in the steady state. In the transient state the flow is weaker, the linear bottom drag is smaller and the northward transport induces the retarding Coriolis force. The resultant force accelerates the zonal flow eastward, eliminating the return flow outside of the region forced by wind. The nonlinear inertial terms are 4 orders of magnitude smaller (not plotted), as expected for the Rossby number $\mathrm{Ro}=O\left(10^{-4}\right)$.
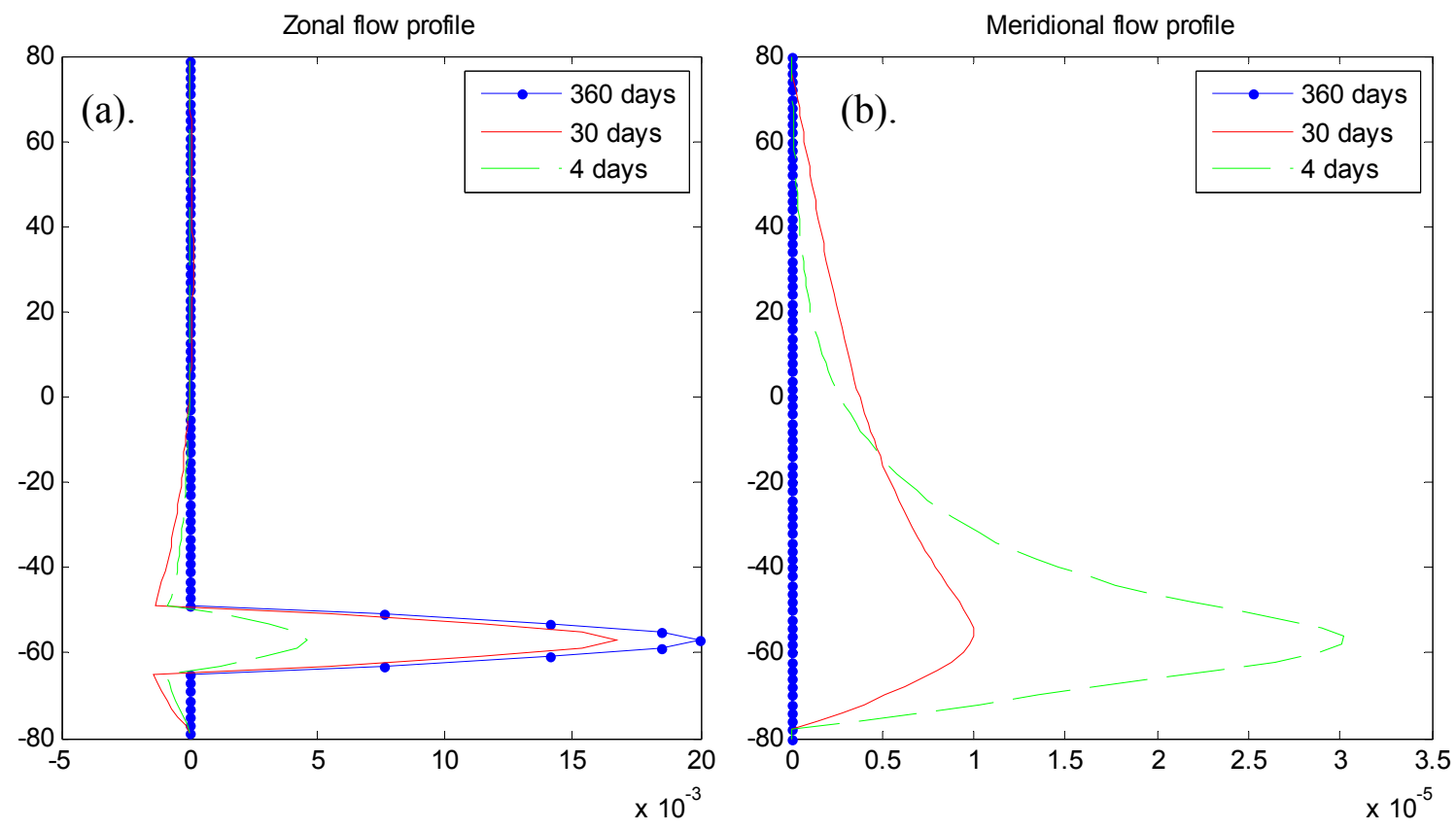

Figure 3.5 Zonal (a) and meridional (b) flow profiles at different simulation times. 


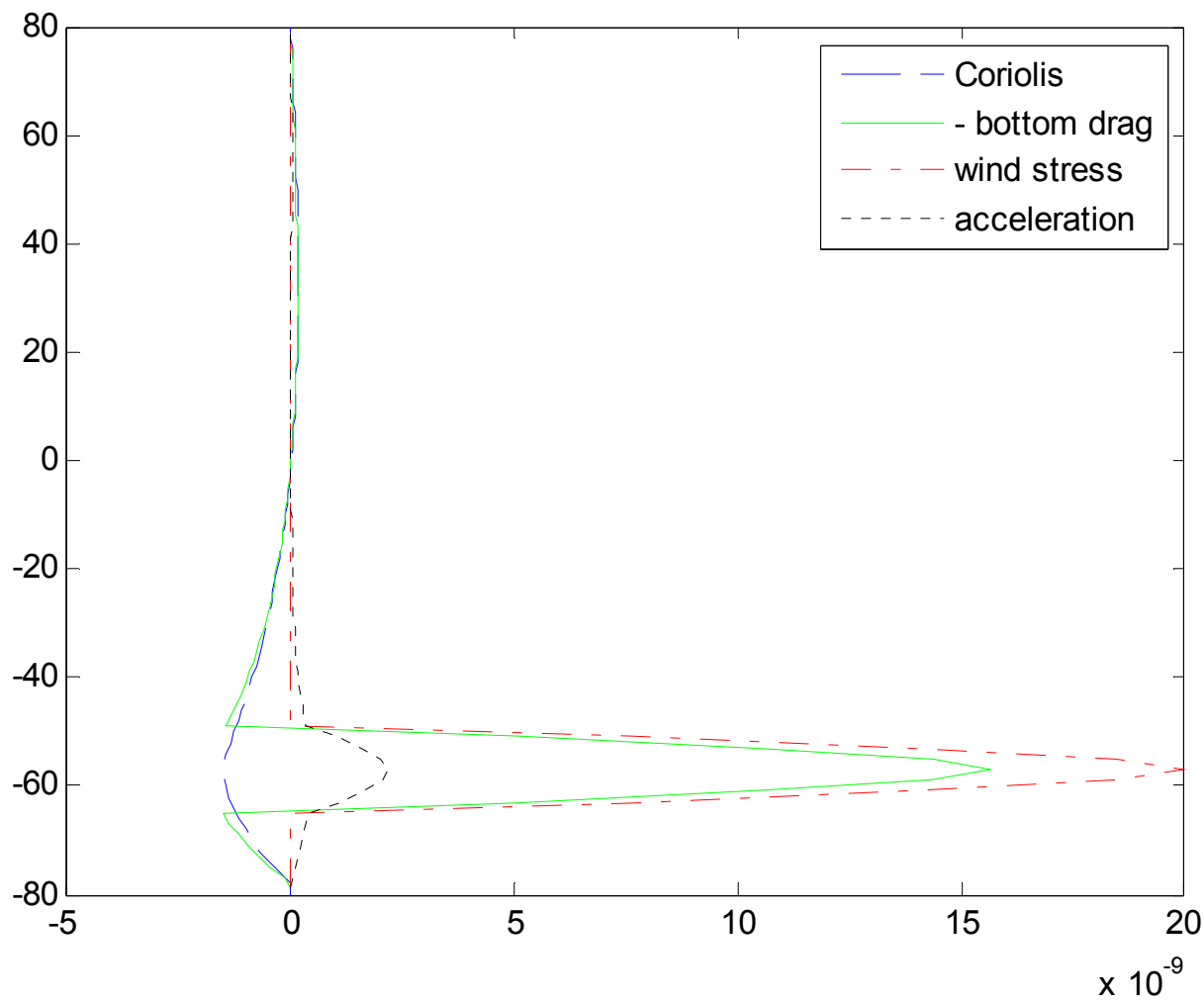

Figure 3.6 Momentum equation terms showing acceleration and forces balance during the transient adjustment state. Bottom drag is plotted multiplied by -1 for convenience of comparison with wind stress. The values are shown in units of $\mathrm{m} / \mathrm{s}^{2}$. 


\subsubsection{Idealized geometry - aqua planet with barrier}

The second step of hierarchical modeling of the ACC in idealized configurations is addition of a meridional barrier to zonal flow. The straight meridional barrier (Figure 3.7) represents an idealized American continent and creates an idealized Drake Passage narrowing. The simulation spins up to a slightly more complex steady state. It still exhibits wind forcing - bottom drag balance, however the zonal flow accelerates in the narrowing at the northern side adjacent to the barrier. The relaxation to steady state is slightly faster than in the aqua planet case, still $O(100)$ days. The total zonal transport at the steady state is slightly weaker $109 \mathrm{~Sv}$. Both results are consistent with slightly larger effective bottom friction $r$ in (3.1.11) and (3.2.1).

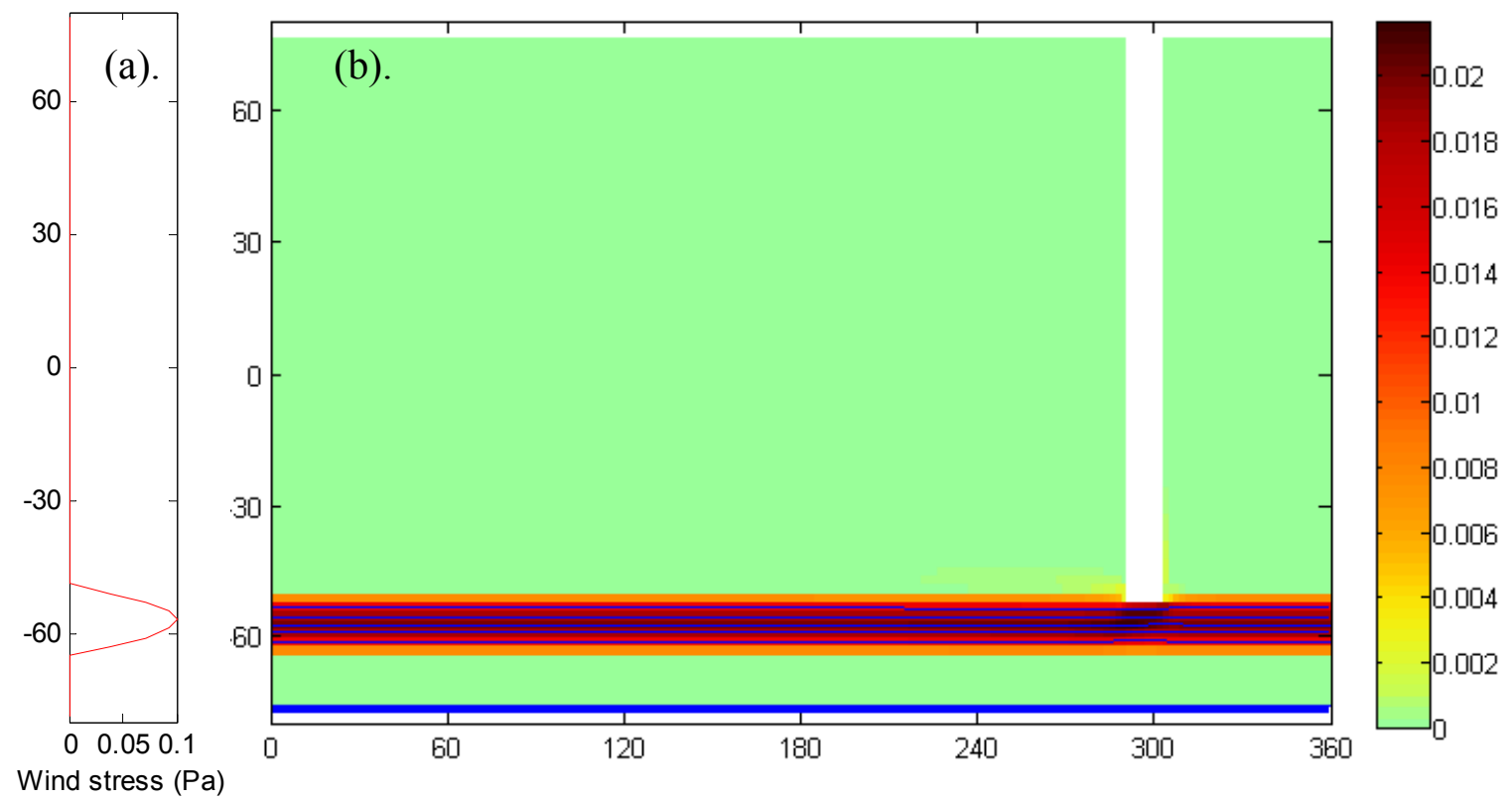

Figure 3.7 Wind stress profile (a) and barotropic flow map (b) shown by the magnitude of 2-D velocity vector (color shades) overlaid with sea surface height contours (blue).

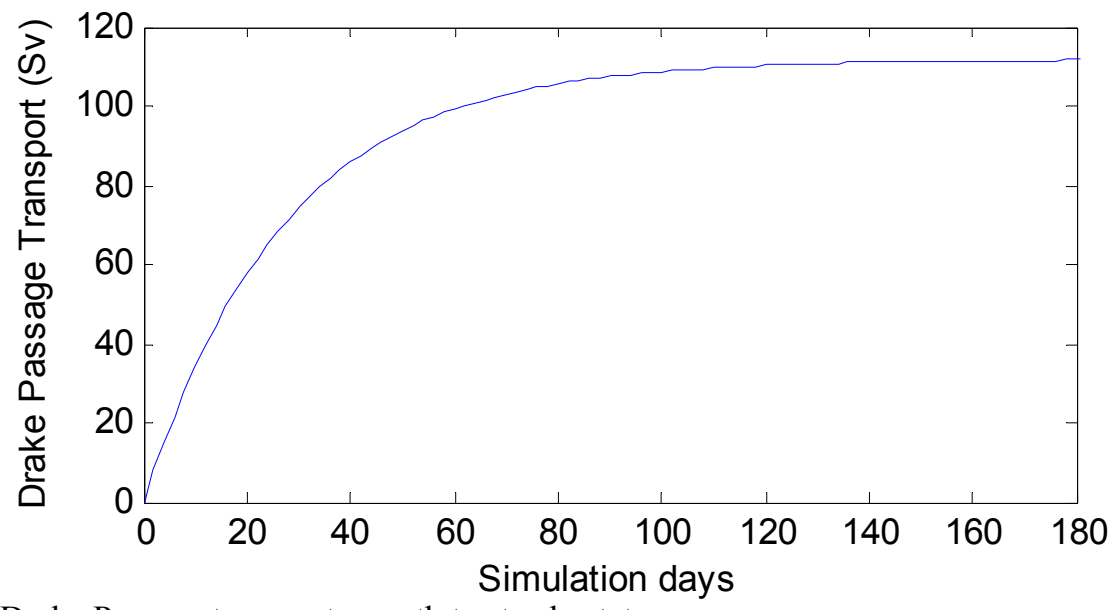

Figure 3.8 Drake Passage transport growth to steady state. 


\subsubsection{Realistic geometry with flat bottom}

The next step towards a realistic ACC configuration is inclusion of the realistic ocean geometry. We use realistic bathymetry data and flatten it globally to depth of $5000 \mathrm{~m}$. The continents outlines remain unchanged and follow the real coastlines. The model spins up to a steady state in $O(100)$ days, yet at a little faster rate than in the two previous idealized configurations. The equilibrium balance of wind forcing and bottom drag is modified by the continental boundaries. The acceleration of the flow in the Drake Passage narrowing is more substantial, accompanied with denser concentration of sea surface elevation contours. Boundary currents appear around continents close to the latitudes of the applied wind stress, and diffusive weak flows emanate from the main ACC jet. The total zonal transport is $100 \mathrm{~Sv}$, slightly smaller than in the aqua planet configurations. The results are consistent with the barotropic theory of ACC (LaCasce and Isachsen 2010) for closed geostrophic contours, predicting flow primarily along the contours and circumpolar transport inversely proportional to bottom drag coefficient. The simulations are also consistent with the developed idealized analytical model scaling with larger effective bottom friction $r$ in (3.1.11) and (3.2.1) due to the geometric blocking effects of the narrower Drake Passage opening.

Barotropic dynamics are an oversimplification of the real ocean physics, resolving only the large space and short time scales of motion. Nonetheless, the utility of the flat bottom barotropic analysis here extends also to the stratified baroclinic dynamics of the fine spatial scale and long period features of the ocean. The linear quasi-geostrophic baroclinic expansion for flat bottom (Section 16.5 in Cushman-Roisin and Beckers 2011) shows that the baroclinic modes dynamics is identical to the barotropic mode with the (external) Rossby radius of deformation replaced by the much smaller internal radii of

deformation. Note, that this equivalence is limited to the Rossby (planetary) wave dynamics and does not apply to our analysis of Kelvin (gravity) waves below. 


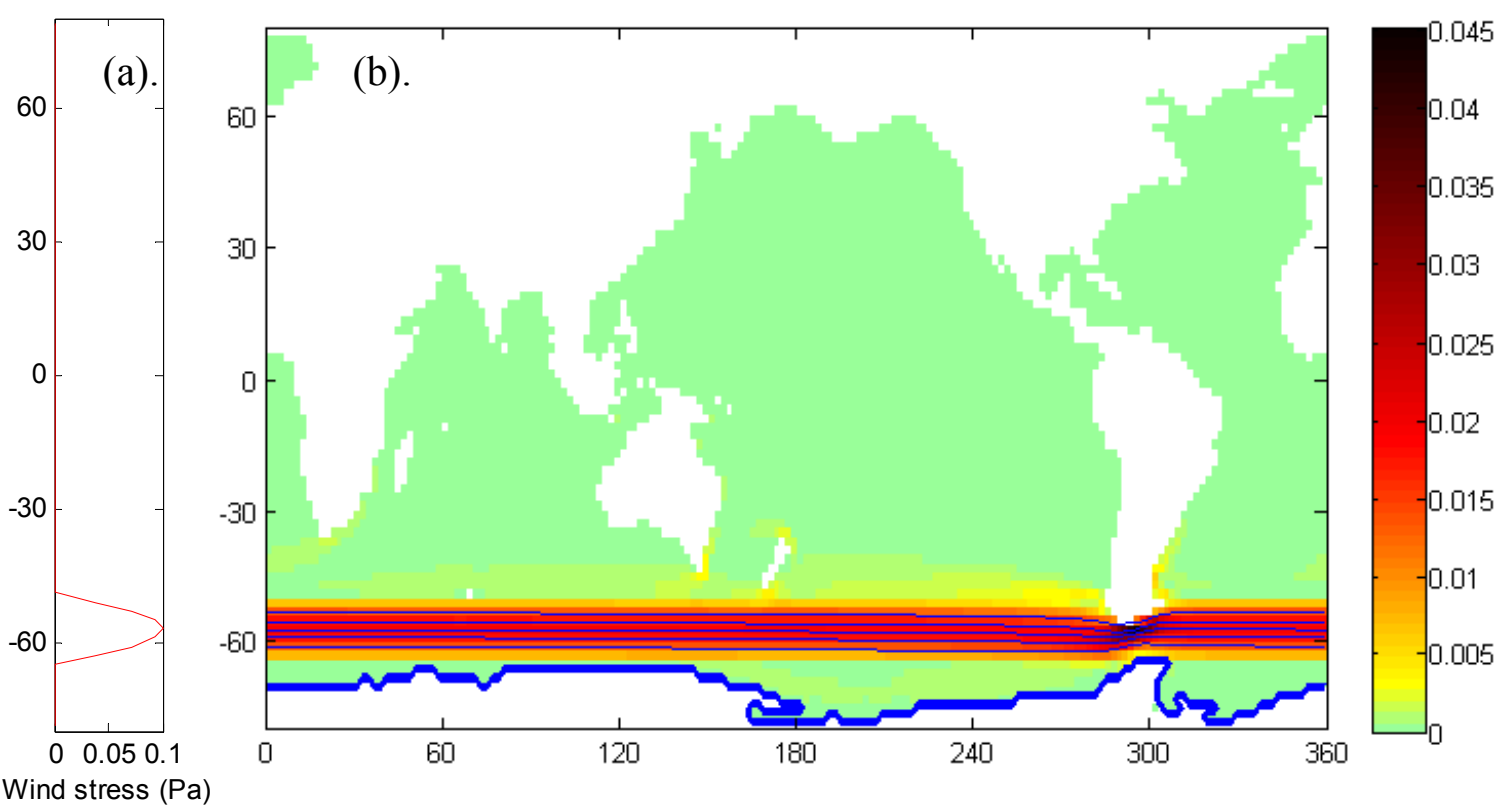

Figure 3.9 Wind stress profile (a) and barotropic flow map (b) shown by the magnitude of 2-D velocity vector (color shades) overlaid with sea surface height contours (blue).

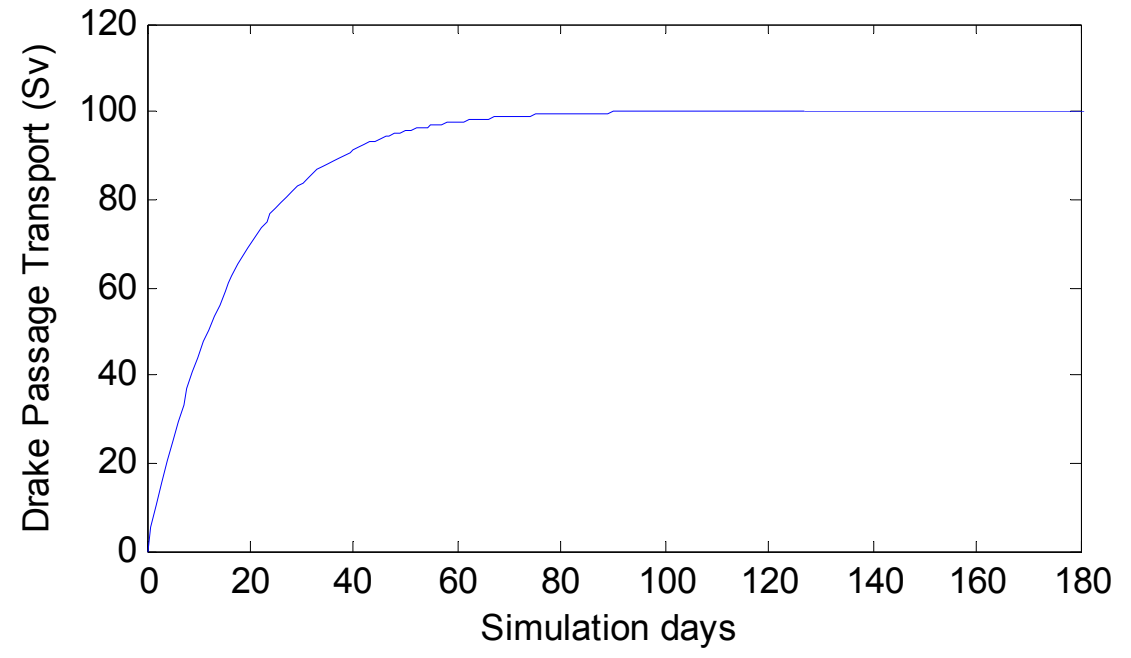

Figure 3.10 Drake Passage transport growth to steady state. 


\subsubsection{Realistic bathymetry}

More realistic barotropic ACC simulation configuration is designed with real bathymetry data. The addition of bottom topography dramatically modifies the geometric patterns of wind driven flow response. This is the expected effect of barotropic flow control by the depth of the water column as the current is steered by the geostrophic contours due to conservation of its potential vorticity, which for small Rossby numbers is given by the depth-normalized Coriolis parameter $f / H$. The shape of the simulated ACC roughly resembles the averaged frontal features in the schematic depiction of the observed ACC (Rintoul et al. 2001), as it meanders across the wind forced latitudes of the Southern Ocean. The pattern is similar to the results of other barotropic simulation studies (Weijer and Gille 2005, Olbers and Lettmann 2007) of wind driven ACC response. LaCasce and Isachsen (2010) explain that with blocked geostrophic contours the flow is "overlycontrolled by topography". The circumpolar jets follow the geostrophic contours over most of the domain, but are effectively blocked by underwater topographic barriers at the Scotian Island Arc and the Kerguelen Plateau. The bottom-steered circulation exhibits gyres with weak cross-contour flow and strong western boundary currents. The resulting circumpolar transport is an order of magnitude weaker (not shown) than its observed values, consistent with the linear barotropic theory of Kamenkovich (1962). The utility of the realistic bathymetry configuration in barotropic modeling is limited because the topography is allowed to have too large effect on the flow, which is unrealistic. Equivalent barotropic models (Krupitsky et al. 1996, LaCasce and Isachsen 2010) reduce the effects of topography and result in more realistic circumpolar transport.

The implementation of these simulations with MITgcm encountered numerical instabilities and required addition of a dissipative numerical scheme for stabilization. We applied the C-D stabilization scheme (Adcroft et al. 1999), which may have added to the blocked geostrophic contours effect resulting in an order of magnitude smaller Drake Passage transport than the observed values. To simulate transport of comparable magnitude to the other configurations in this thesis, the supplied wind forcing momentum input was increased about an order of magnitude (Figure 3.11). 
The resulting realistic bathymetry simulations reach the steady state faster, in order of 30 days. This is consistent with our scaling of the spin down time $H / r$, because the realistic bathymetry domain is shallower on average compared to $5000 \mathrm{~m}$ depth in the flat bottom configurations. The resulting magnitude of the total zonal transport in the steady state is $110 \mathrm{~Sv}$.

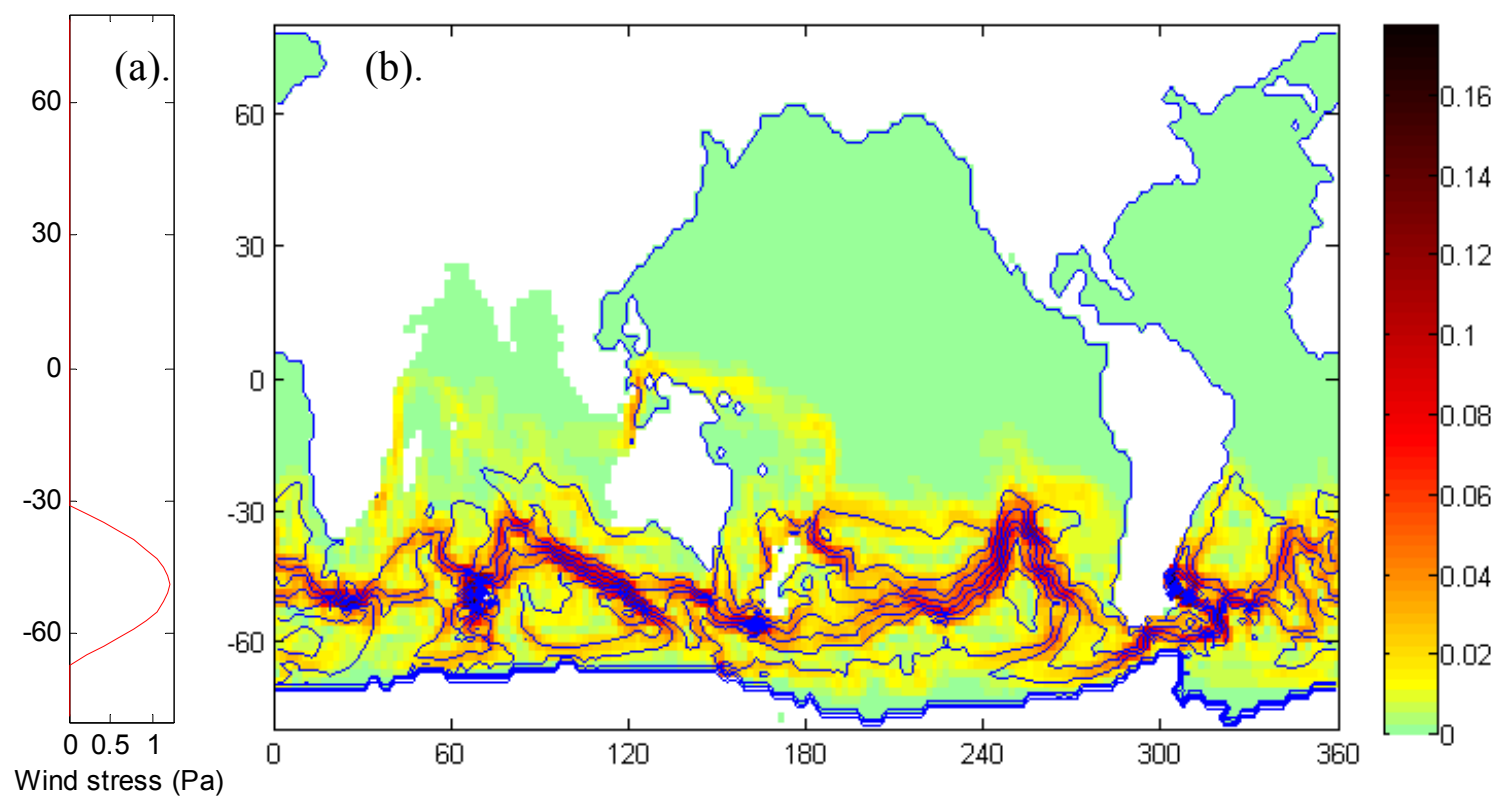

Figure 3.11 Wind stress profile (a) and barotropic flow map (b) shown by the magnitude of 2-D velocity vector (color shades) overlaid with sea surface height contours (blue).

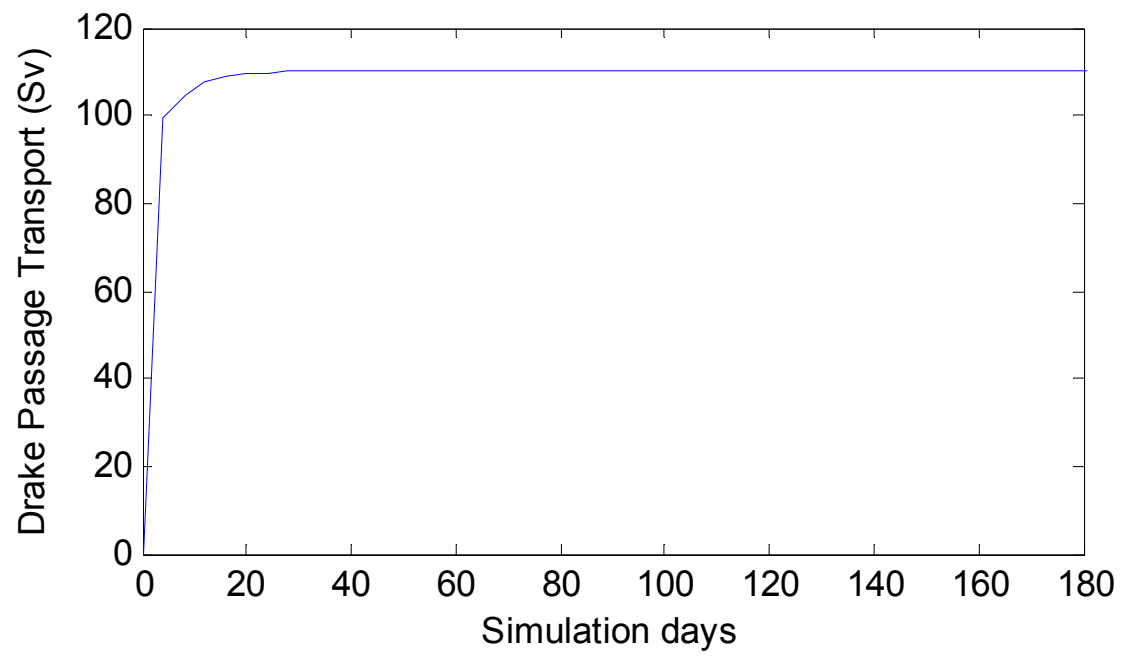

Figure 3.12 Drake Passage transport growth to steady state. 


\section{UQ analysis with analytical ODE model}

\subsection{Steady state analysis}

We start with implementation of the UQ system for the analytical ODE model due to its simplicity to analyze and the convenience to confirm analytically the computational results, while resolving the principal dynamical processes and thus involving the some of the challenges relevant to the full oceanographic state estimation problem. We quantify the uncertainty of the forced exponential relaxation dynamics governed by

$$
\frac{d u}{d t}=-R u+F
$$

The generalized control vector can be defined to include only forcing and dissipation parameters

$$
\mathbf{x}_{1}=[F, R]^{T}
$$

Or it can include also the initial conditions

$$
\mathbf{x}_{2}=\left[F, R, u_{0}\right]^{T}
$$

The output of the model can be defined as the trajectory of its solution - in this case the vector of values $u(t)$ at a range of times e.g. $\left[t_{0}, t\right]$, or just a single scalar value at some chosen time. Both configurations were successfully implemented numerically. For simplicity of the analytical derivation we first discuss the later choice, defining the model output as the steady state flow rate $u_{\infty}$ after sufficiently long integration

$$
\boldsymbol{M}(\mathbf{x})=u(t=\infty) \equiv u_{\infty}
$$

For verification of the UQ system both the assimilated observations and the estimated target variable are set to the steady state flow

$$
\begin{gathered}
\mathbf{y}=u_{o b s}=u_{\infty} \\
\mathbf{z}=u_{\infty}
\end{gathered}
$$

The cost function for a single scalar model output is

$$
J=\frac{1}{2}(\boldsymbol{M}(\mathbf{x})-\mathbf{y})^{T} \mathbf{R}^{-1}(\boldsymbol{M}(\mathbf{x})-\mathbf{y})=\frac{1}{2} \frac{\left(u_{\infty}(\mathbf{x})-u_{o b s}\right)^{2}}{\Delta u^{2}}
$$

where $\Delta u$ is the assumed observation uncertainty. 
The Jacobian matrices of the modeled variable, here the steady state flow

$$
u_{\infty}=F / R
$$

can be written out explicitly in both control spaces

$$
\begin{gathered}
\frac{\partial u_{\infty}}{\partial \mathbf{x}_{1}{ }^{T}}=\left[\begin{array}{ll}
\frac{\partial u_{\infty}}{\partial F} & \frac{\partial u_{\infty}}{\partial R}
\end{array}\right]=\left[\begin{array}{ll}
\frac{1}{R} & \frac{-F}{R^{2}}
\end{array}\right] \\
\frac{\partial u_{\infty}}{\partial \mathbf{x}_{2}{ }^{T}}=\left[\begin{array}{lll}
\frac{\partial u_{\infty}}{\partial F} & \frac{\partial u_{\infty}}{\partial R} & \frac{\partial u_{\infty}}{\partial u_{0}}
\end{array}\right]=\left[\begin{array}{lll}
\frac{1}{R} & \frac{-F}{R^{2}} & 0
\end{array}\right]
\end{gathered}
$$

Note that the steady state Jacobian does not depend on the initial conditions. Moreover, the sensitivity of the steady state to the initial conditions is zero, confirming that the asymptotic steady state solution "forgets" completely the initial conditions of the ODE. The sensitivity of the steady state flow to wind forcing is positive and stronger for smaller friction. The sensitivity of the steady state flow to the friction parameter is, as expected, negative. Interesting results are that for a stronger forced system the negative sensitivity to friction is linearly stronger, while for a stronger friction parameter this negative sensitivity is weaker. Both forcing and friction sensitivities are nonlinear functions of the friction parameter, and since these first derivatives are not just constants the model is classified as nonlinear. While the Hessian matrix of a linear model must vanish, the Hessian of our nonlinear model does not. Written explicitly for $\mathbf{x}_{1}$ controls the Hessian of the modeled variable is

$$
\frac{\partial^{2} u_{\infty}}{\partial \mathbf{x}_{1}{ }^{2}}=\left[\begin{array}{ll}
\frac{\partial^{2} u_{\infty}}{\partial F^{2}} & \frac{\partial^{2} u_{\infty}}{\partial R \partial F} \\
\frac{\partial^{2} u_{\infty}}{\partial R \partial F} & \frac{\partial^{2} u_{\infty}}{\partial R^{2}}
\end{array}\right]=\left[\begin{array}{cc}
0 & \frac{-1}{R^{2}} \\
\frac{-1}{R^{2}} & \frac{2 F}{R^{3}}
\end{array}\right]
$$

The explicit form of the model Hessian in the $\mathbf{x}_{2}$ control space is given simply by augmenting this 2 by 2 matrix with zeros, because all the derivatives with respect to the initial conditions controls are zero in the steady state.

One should not confuse the Hessian of the model (4.1.11) with the Hessian matrix of the UQ analysis - given by the Hessian of the misfit cost function. The Hessian of the misfit (4.1.7) can be calculated analytically either directly term by term or using the formula (2.6.2), written explicitly 


$$
\begin{aligned}
& \frac{\partial^{2} J}{\partial \mathbf{x}_{1}{ }^{2}}=\left(\frac{\partial \boldsymbol{M}\left(\mathbf{x}_{1}\right)}{\partial \mathbf{x}_{1}{ }^{T}}\right)^{T} \mathbf{R}^{-1}\left(\frac{\partial \boldsymbol{M}\left(\mathbf{x}_{1}\right)}{\partial \mathbf{x}_{1}{ }^{T}}\right)+\left(\frac{\partial^{2} \boldsymbol{M}\left(\mathbf{x}_{1}\right)}{\partial \mathbf{x}_{1}{ }^{2}}\right)^{T} \mathbf{R}^{-1}\left(\boldsymbol{M}\left(\mathbf{x}_{1}\right)-u_{o b s}\right)= \\
& =\left[\begin{array}{c}
\frac{\partial u_{\infty}}{\partial F} \\
\frac{\partial u_{\infty}}{\partial R}
\end{array}\right]\left(\Delta u^{2}\right)^{-1}\left[\begin{array}{ll}
\frac{\partial u_{\infty}}{\partial F} & \frac{\partial u_{\infty}}{\partial R}
\end{array}\right]+\left[\begin{array}{ll}
\frac{\partial^{2} u_{\infty}}{\partial F^{2}} & \frac{\partial^{2} u_{\infty}}{\partial R \partial F} \\
\frac{\partial^{2} u_{\infty}}{\partial R \partial F} & \frac{\partial^{2} u_{\infty}}{\partial R^{2}}
\end{array}\right] \cdot\left(\Delta u^{2}\right)^{-1}\left(u_{\infty}\left(\mathbf{x}_{1}\right)-u_{o b s}\right)
\end{aligned}
$$

Note, that the second RHS term of the cost function Hessian does include the contribution of the model Hessian (4.1.11). Yet, since the misfits are zero in our setup, the second Hessian term disappears. The remaining term is commonly termed the linearized Hessian (Losch and Wunsch 2003), although in general it is not a result of asymptotically consistent Hessian linearization. That can be explained because the other (nonlinear) term appears only for nonlinear models and vanishes for linear models. We highlight that linearity of the model in this context is different from the linearity of the ODE equation (4.1.1) that constitutes the model $\boldsymbol{M}(\mathbf{x})$. The model $\boldsymbol{M}(\mathbf{x})$ is nonlinear because of the nonlinear dependence of its output on the controls $\mathbf{x}$, or equivalently, because its Jacobian (4.1.10) is not constant, i.e. that model Hessian (4.1.11) is not zero.

Whereas in this thesis we focus only on the implementation of the identical twins setup, from now on we concentrate only on the so-called linearized Hessian. Written explicitly in the $\mathbf{x}_{1}$ control space the linearized Hessian of misfits is given by

$$
\left(\frac{\partial^{2} J}{\partial \mathbf{x}_{1}^{2}}\right)_{L}=\left[\begin{array}{c}
\frac{\partial u_{\infty}}{\partial F} \\
\frac{\partial u_{\infty}}{\partial R}
\end{array}\right]\left(\Delta u^{2}\right)^{-1}\left[\begin{array}{ll}
\frac{\partial u_{\infty}}{\partial F} & \frac{\partial u_{\infty}}{\partial R}
\end{array}\right]=\frac{1}{\Delta u^{2}}\left[\begin{array}{ll}
\left(\frac{\partial u_{\infty}}{\partial F}\right)^{2} & \frac{\partial u_{\infty}}{\partial R} \frac{\partial u_{\infty}}{\partial F} \\
\frac{\partial u_{\infty}}{\partial R} \frac{\partial u_{\infty}}{\partial F} & \left(\frac{\partial u_{\infty}}{\partial R}\right)^{2}
\end{array}\right]=\frac{1}{\Delta u^{2}}\left[\begin{array}{cc}
\frac{1}{R^{2}} & \frac{-F}{R^{3}} \\
\frac{-F}{R^{3}} & \frac{F^{2}}{R^{4}}
\end{array}\right]
$$

Compare this Hessian matrix to the Hessian of the model (4.1.11). They have different structure and different properties. While both are symmetric (which is a basic property of any Hessian), the Hessian of misfits is positive-semidefinite and singular, while the model Hessian is regular and indefinite ${ }^{11}$. Essentially, only the later involves the second derivatives of the model, the former is constructed only of products of the first derivatives.

${ }^{11}$ The eigenvalues of (4.1.11) are $\lambda_{1,2}=\frac{u_{\infty}}{R^{2}} \pm \frac{1}{R^{2}} \sqrt{u_{\infty}^{2}+1}$ always indefinite, for $u_{\infty} \ll 1 \quad \lambda_{1,2}= \pm \frac{1}{R^{2}}$. 
It is important to develop intuition for the construction and the detailed structure of the analytically derived Hessian of misfits as a guide to understanding the Hessians of much more complex configurations. Note that the Hessian matrix (4.1.13) is essentially a scaled outer product of vectors given by the sensitivity Jacobians above (4.1.9). More precisely, adopting the definition of a gradient as the transpose of the Jacobian matrix (Magnus and Neudecker 1988 p. 87), the linearized Hessian of the cost function is the outer product of the gradients of the scalar output of the model scaled by its observed uncertainty. In the multivariate model output case, the Hessian is essentially the sum of the outer products of gradients of the separate scalar outputs scaled by their observed uncertainty variances. This, of course, is exact only if the observed uncertainty covariance matrix $\mathbf{R}$ is diagonal, otherwise the algebra is more involved. If the gradients of the scalar model outputs are orthogonal and $\mathbf{R}$ is diagonal, then this explicit expression for the Hessian is exactly equivalent to the eigenvalue decomposition form (2.7.1).

Understanding the dependence of the linearized Hessian on Jacobian vectors (or gradients) of model outputs is critical for design of uncertainty quantification experiments. The singularity of the Hessian is a direct result of linear dependence of the Jacobians of separate model outputs. In our example (4.1.13) the Hessian is given by the outer product of only one vector and thus its rank is equal one. One could add another scalar model output to the misfit cost function in attempt to increase its rank and remove the singularity. This would succeed only if the new scalar model output (and the corresponding observation) are independent of the first one. For example, in our ODE system if one adds another observation of the converged steady state flow, say one day later, the additional Jacobian will be identical to the first one and will not increase the rank of the Hessian. One could have implemented a complex cost function with many additional observations, but if these are not dynamically independent from the first observation, despite an increased computational complexity there would be little benefit to regularizing the Hessian. Note, that our discussion leads to a formal definition of dynamical independence of observations - in terms of the linear independence of the corresponding Jacobians. In the design of an optimal observation system one should attempt to include only the minimum amount of dynamically independent observations, to keep the computational complexity down, while maximizing the rank of the Hessian. 
This is equivalent to designing an observational system to maximize the range and minimize the nullspace of the uncertainty quantification problem.

Similarly, the dynamical independence of observations is the basis of the reduced order numerical algorithm for the Hessian inversion and uncertainty reduction (Section 2.7). The eigenvalue decomposition of the Hessian (2.7.1) separates the orthogonal linearly independent Hessian eigenvectors, which are equivalent to the independent gradients in (4.1.13) combined by the weighted sum of their outer products. Discarding the nullspace of the Hessian removes those outer product matrices who do not contribute to the sum and do not constrain the posterior uncertainty in (2.7.5).

The pseudo-inverse of the Hessian can be constructed analytically by treating (4.1.13) as the spectral decomposition (2.7.1) with the only nonzero eigenvalue

$$
\lambda_{1}=\frac{G^{2}}{\Delta u^{2}}
$$

Here $G$ is the normalizing factor given by the norm of the gradient given by transpose of (4.1.9). Pseudo-inverse of the Hessian by substitution into (2.7.2) is given by

$$
\mathbf{H}^{+}=\frac{\Delta u^{2}}{G^{4}}\left[\begin{array}{l}
\frac{\partial u_{\infty}}{\partial F} \\
\frac{\partial u_{\infty}}{\partial R}
\end{array}\right]\left[\begin{array}{ll}
\frac{\partial u_{\infty}}{\partial F} & \frac{\partial u_{\infty}}{\partial R}
\end{array}\right]=\Delta u^{2}\left(\frac{R^{4}}{R^{2}+F^{2}}\right)^{2}\left[\begin{array}{cc}
\frac{1}{R^{2}} & \frac{-F}{R^{3}} \\
\frac{-F}{R^{3}} & \frac{F^{2}}{R^{4}}
\end{array}\right]
$$

This rank-1 matrix constrains the controls, either in 2 or 3 dimensional control spaces, only in one direction given by the gradient vector. This direction is represented by the covariances, which are negative, meaning that knowing the steady state flow constrains the forcing and the friction to be less negatively correlated. In other words, they are constrained to co-vary positively. This is intuitively clear by looking at (4.1.8), because for a given steady state flow an uncertain positive perturbation of wind forcing must be balanced by the corresponding positive perturbation of bottom drag. We learn from this example that seeing negative off-diagonal terms in covariance matrix doesn't necessarily imply negative correlation when the covariance matrix is singular. The unconstrained orthogonally complementary directions in control space remain unbound with infinite uncertainty. In our example in 2 dimensional space, this is the vector orthogonal to the sensitivity gradient vector and it is oriented such that forcing and friction are positively 
correlated. Another way to see this is to consider the prior to posterior uncertainty reduction formula (2.7.6), which for a prior given by the identity matrix can be written analytically

$$
\mathbf{P}=\mathbf{I}-\left[\begin{array}{c}
\frac{\partial u_{\infty}}{\partial F} \\
\frac{\partial u_{\infty}}{\partial R}
\end{array}\right]\left(\frac{\Delta u^{2}}{G^{2}}+1\right)^{-1}\left[\begin{array}{ll}
\frac{\partial u_{\infty}}{\partial F} & \frac{\partial u_{\infty}}{\partial R}
\end{array}\right]=\mathbf{I}-\frac{1}{\Delta u^{2}+G^{2}}\left[\begin{array}{cc}
\frac{1}{R^{2}} & \frac{-F}{R^{3}} \\
\frac{-F}{R^{3}} & \frac{F^{2}}{R^{4}}
\end{array}\right]
$$

We see that reducing the uncorrelated prior uncertainty by subtracting negative offdiagonal terms, we end up with a positive off-diagonal posterior covariance terms, indicating positive posterior correlation of wind and bottom friction.

One can confirm the consistency of the inverse and forward projection of uncertainty, by projecting the calculated Hessian pseudo-inverse (4.1.15) forward, back to the space of the estimated steady state flow (4.1.6). This was verified both - numerically, with the developed AD machinery, and analytically as shown below.

$$
\mathbf{g}^{T} \mathbf{H}^{+} \mathbf{g}=\left[\begin{array}{ll}
\frac{\partial u_{\infty}}{\partial F} & \frac{\partial u_{\infty}}{\partial R}
\end{array}\right] \mathbf{H}^{+}\left[\begin{array}{c}
\frac{\partial u_{\infty}}{\partial F} \\
\frac{\partial u_{\infty}}{\partial R}
\end{array}\right]=\Delta u^{2}
$$

\subsection{Time resolving analysis}

The previous section limited the analysis of the UQ system only to the steady state solution of the ODE model. However, the essential feature of the general Ocean State Estimation problem is resolving the temporal evolution of the solution and the accompanying UQ system must resolve the time dependence of the uncertainties. This time dependence increases the complexity of the analysis, as was acknowledged and left unexplored by Losch and Wunsch (2003), but also provides a much richer understanding of the dynamics of the solution and its uncertainty.

The closed form analytical solution of the ODE model (4.1.1) allows a convenient and explicit derivation of the time dependent form of the UQ system objects. One can analytically differentiate the solution

$$
u(t)=u_{0} e^{-R\left(t-t_{0}\right)}+\frac{F}{R}\left(1-e^{-R\left(t-t_{0}\right)}\right)
$$


to obtain the components of the Jacobian or sensitivity vector. Since the solution depends linearly on the forcing parameter, the partial derivative with respect to forcing is trivial

$$
\frac{\partial u(t)}{\partial F}=\frac{1}{R}\left(1-e^{-R\left(t-t_{0}\right)}\right)
$$

The sensitivity to forcing grows from zero and relaxes exponentially to the asymptotic steady state value that was discussed in the previous section (4.1.9). The sensitivity remains always positive - for stronger wind input the flow response is always stronger. The fact that the sensitivity of the response grows with time can be explained as the gradual adjustment of the flow to the steadily supplied wind forcing.

The sensitivity of the solution to the friction parameter is more complex.

$$
\frac{\partial u(t)}{\partial R}=-u_{0} R e^{-R\left(t-t_{0}\right)}-\frac{F}{R^{2}}\left(1-e^{-R\left(t-t_{0}\right)}\right)+F e^{-R\left(t-t_{0}\right)}=R\left(\frac{F}{R}-u_{0}\right) e^{-R\left(t-t_{0}\right)}-\frac{F}{R^{2}}\left(1-e^{-R\left(t-t_{0}\right)}\right)
$$

The three separate terms (before combining the exponentials) can be identified as - (1) the sensitivity to the transient decay of the initial conditions, (2) the sensitivity to the adjustment to the steady state due to the steady state balance sensitivity, and (3) the sensitivity to the transient growth due to the sensitivity of the growth rate. The first of the three is negative (for positive initial conditions) and exponentially decreases in magnitude, meaning that for stronger friction the transient flow decays faster. The second term is always negative, it starts from zero growing to the negative steady state sensitivity discussed in the previous section (4.1.9). The third term is positive as it captures the sensitivity to the growth rate - the stronger the friction the faster the system adjust to the supplied forcing. But as the adjustment slows down with time, this positive sensitivity exponentially disappears.

The two transient friction sensitivity terms can be combined to separate the transient disappearing effects from the growing steady state effects in the RHS of (4.2.3). These two competing processes are the key to understanding the complex temporal evolution of the UQ analysis results in the following chapter. The current result shows that if the integration starts from the steady state initial conditions (4.1.8), then the transient decaying signals cancel out and the sensitivity evolves from zero to its steady state. In alternative simulation, if integrating from rest the sensitivity starts evolving from a non- 
zero initial value and it is possible to temporarily find the system in overall positive sensitivity to friction, which is a contra-intuitive result.

Next we examine the sensitivity to the initial conditions $u_{0} \equiv u\left(t_{0}\right)$. Written analytically, it takes a simple exponentially decaying form independent of forcing or initial conditions themselves:

$$
\frac{\partial u(t)}{\partial u\left(t_{0}\right)}=e^{-R\left(t-t_{0}\right)}=e^{R\left(t_{0}-t\right)}
$$

The sensitivity is positive - larger initial flow leads to larger transient solution, while the system is exponentially "forgetting" the effects of the initial conditions.

The sensitivity of flow response to the initial flow magnitude is a unique sensitivity term, because it is a derivative of one physical variable by itself but at a different time. It highlights the relation between two tangent linear perturbations of one variable shifted in time and its adjoint - given by the ratio of these two perturbations. More precisely, the adjoint of $u(t)$ at time $t=t_{0}$ is defined ${ }^{12}$ as the sensitivity term (4.2.4). The time shift between the two perturbations can be referred to as a positive forward in time shift $t-t_{0}$ or as a negative backward in time shift $t_{0}-t$. For the convenience of the discussion of the adjoint model results, we formally define the adjoint time

$$
\tau \equiv t_{0}-t
$$

We note, that because here we examine the derivatives of an autonomous ODE (4.1.1) which solution does not depend separately on the initialization time $t_{0}$ and the forward time $t$, the derivatives also do not depend separately on the initialization time but only on the time shift. Nonetheless, in general the Jacobian (and the gradient) may depend on each of these two time separately

$$
\mathbf{g}^{T}\left(t_{0}, t\right)=\frac{\partial u(t)}{\partial \mathbf{x}_{2}{ }^{T}}=\left[\begin{array}{lll}
\frac{\partial u}{\partial F} & \frac{\partial u}{\partial R} & \frac{\partial u}{\partial u_{0}}
\end{array}\right]
$$

Example of such case would be a time varying wind forcing, e.g. due to the annual variability cycle. Moreover, the forward time $t$ may be in general different from the time

\footnotetext{
${ }^{12}$ Definition of the adjoint variable $\delta u^{*}\left(t_{0}\right)$ of the variable $u\left(t_{0}\right)$ and its tangent-linear perturbation $\delta u\left(t_{0}\right)$ is given by $\delta u(t)=\left\langle\delta u^{*}\left(t_{0}\right), \delta u\left(t_{0}\right)\right\rangle$.
} 
of the assimilated observations $t_{\mathrm{a}}$. The posterior covariance constrained by the observation uncertainty $\mathbf{R}\left(t_{\mathrm{a}}\right)$ would in general depend on the initialization and the assimilation times $\mathbf{P}\left(t_{0}, t_{a}\right)$ and so is the inverse of the assimilation Hessian

$$
\mathbf{H}^{+}\left(t_{0}, t_{a}\right)=\left[\begin{array}{ccc}
\sigma_{F}^{2} & \sigma_{F R} & \sigma_{F u_{0}} \\
\sigma_{F R} & \sigma_{R}^{2} & \sigma_{R u_{0}} \\
\sigma_{F u_{0}} & \sigma_{R u_{0}} & \sigma_{u_{0}}^{2}
\end{array}\right]
$$

The eventual result of forward uncertainty projection to time $t$ of controls defined for initial conditions at time $t_{0}$ and constrained by uncertainty assimilated at time $t_{\mathrm{a}}$ would lead to the triple time dependence

$$
\sigma_{z}^{2}\left(t_{0}, t_{a}, t\right)=\mathbf{g}^{T} \mathbf{P g}=\mathbf{g}^{T} \mathbf{P}_{0} \mathbf{g}-\mathbf{g}^{T} \mathbf{\Gamma} \mathbf{g}
$$

Here we introduced another symbol, $\boldsymbol{\Gamma}$, for the posterior uncertainty reduction matrix. Based on the definition of the unregularized Hessian (2.6.2) and the matrix inversion lemma applied to $\mathbf{P}^{-1}=\mathbf{P}_{0}^{-1}+\mathbf{H}$, the uncertainty reduction matrix can be written out

$$
\boldsymbol{\Gamma}\left(t_{0}, t_{a}\right)=\mathbf{P}_{0}\left(\mathbf{H}^{-1}+\mathbf{P}_{0}\right)^{-1} \mathbf{P}_{0}
$$

Note that for ill-posed problems, when Hessian $\mathbf{H}$ is not invertible, the expression (4.2.9) is undefined. Nonetheless, the uncertainty reduction matrix can be written out based on the reduced rank Hessian decomposition (2.7.6)

$$
\boldsymbol{\Gamma}\left(t_{0}, t_{a}\right)=\mathbf{P}_{0} \mathbf{V}\left(\boldsymbol{\Lambda}^{-1}+\mathbf{V}^{T} \mathbf{P}_{0} \mathbf{V}\right)^{-1} \mathbf{V}^{T} \mathbf{P}_{0}
$$

In the current autonomous system analysis the projected target uncertainty result depends explicitly only on the time shifts, and since both the forward and the backward projections of uncertainty are governed by the adjoint Jacobian transformations, the result can be written as $\sigma_{z}^{2}\left(\tau_{a}, \tau\right)$ with the adjoint assimilation time defined

$$
\tau_{a} \equiv t_{0}-t_{a}
$$

We can now proceed to analyze the adjoint dynamics of inverse and forward uncertainty propagation. The combined analytical sensitivity (adjoint) vector of the ODE model solution in the $\mathbf{x}_{2}$ control space can be written in the row vector form of the Jacobian transformation matrix 


$$
\frac{\partial u(t)}{\partial \mathbf{x}_{2}^{T}}=\left[\begin{array}{lll}
\frac{\partial u}{\partial F} & \frac{\partial u}{\partial R} & \frac{\partial u}{\partial u_{0}}
\end{array}\right]=\left[\begin{array}{c}
\frac{1}{R}\left(1-e^{-R\left(t-t_{0}\right)}\right) \\
R\left(\frac{F}{R}-u_{0}\right) e^{-R\left(t-t_{0}\right)}-\frac{F}{R^{2}}\left(1-e^{-R\left(t-t_{0}\right)}\right) \\
e^{-R\left(t-t_{0}\right)}
\end{array}\right]^{T}
$$

Furthermore, one can analytically construct the linearized Hessian matrix of misfits by the outer product of the adjoints as discussed in the section above (4.1.13). Here, to save space, we present the Hessian for the case of the ODE model integration from the steady state initial conditions, which is also the configuration of the numerical model results discussed in the following chapter.

$$
\left(\frac{\partial^{2} J}{\partial \mathbf{x}_{2}^{2}}\right)_{L}=\frac{1}{\Delta u^{2}}\left[\begin{array}{ccc}
\frac{1}{R^{2}}\left(1-e^{-R\left(t_{a}-t_{0}\right)}\right)^{2} & -\frac{F}{R^{3}}\left(1-e^{-R\left(t_{a}-t_{0}\right)}\right)^{2} & \frac{1}{R}\left(1-e^{-R\left(t_{a}-t_{0}\right)}\right) e^{-R\left(t_{a}-t_{0}\right)} \\
-\frac{F}{R^{3}}\left(1-e^{-R\left(t_{a}-t_{0}\right)}\right)^{2} & \frac{F^{2}}{R^{4}}\left(1-e^{-R\left(t_{a}-t_{0}\right)}\right)^{2} & -\frac{F}{R^{2}}\left(1-e^{-R\left(t_{a}-t_{0}\right)}\right) e^{-R\left(t_{a}-t_{0}\right)} \\
\frac{1}{R}\left(1-e^{-R\left(t_{a}-t_{0}\right)}\right) e^{-R\left(t_{a}-t_{0}\right)} & -\frac{F}{R^{2}}\left(1-e^{-R\left(t_{a}-t_{0}\right)}\right) e^{-R\left(t_{a}-t_{0}\right)} & e^{-2 R\left(t_{a}-t_{0}\right)}
\end{array}\right]
$$

The analytical form explicitly shows the structure and properties of the ODE misfit Hessian matrix, which are also expected to be reflected in the results of the more complex numerical model. The Hessian is a singular, rank one matrix. The rank and the range of the Hessian are given by the number and the span of the independent observations Jacobians. The various scalar components of the Hessian matrix have different time evolution profiles (see Figure 4.1), resulting from the cross-products of the Jacobian components. The components associated with the uncertainty of the forcing and friction parameters are growing from zero exponentially relaxing to their steady state values. All the components associated with initial conditions uncertainty vanish exponentially, indicating that after sufficiently long model integration time the observations do not constrain the uncertainty of the initial conditions. Nonetheless, interesting transient crossterms emerge temporarily coupling the initial conditions with forcing and friction parameters. These grow from zero and eventually vanish, due to the transient amplification of the negative superposition of two exponentially decaying modes which fully cancel each other at $t_{0}$ and at the steady state, given by $e^{\tau}\left(1-e^{\tau}\right)=e^{\tau}-e^{2 \tau}$. Such 
cross-coupling of physical variables due to the quadratic combination of sensitivities in the Hessian leads to correlation of uncertainties, which our method allows to identify and which are shown to dominate the uncertainty in ocean state estimation.

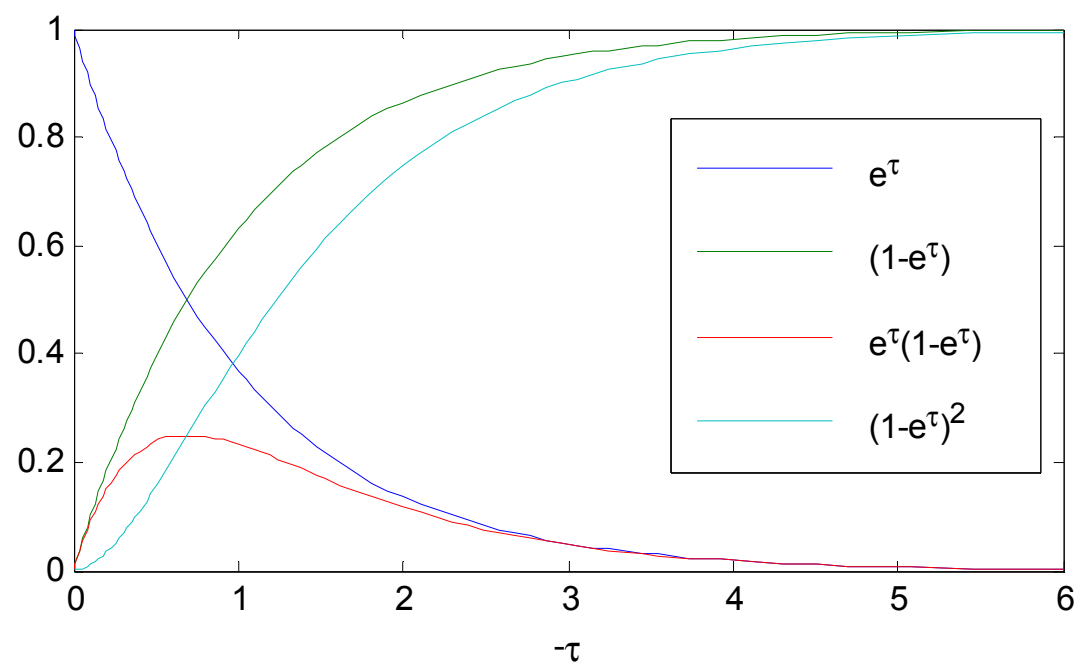

Figure 4.1: Time evolution profiles of scalar components of the Hessian matrix compared to the evolution profiles of the components of the gradient, shown as function of forward assimilation time, which is minus adjoint assimilation time for $t_{0}=0$. (In this figure $\tau$ stands for $\tau_{a}$ ).

One can further utilize the explicit form of the analytical UQ solution to gain better understanding of the physical mechanisms of uncertainty evolution in wind-bottom friction adjustment problem. Following the procedure as in the previous section, we can construct analytically the pseudo-inverse of the Hessian. Note, that the only nonzero eigenvalue is given by (4.1.14). Here $G$ is the normalizing factor given by the norm of the vector (4.2.12) for the adjoint assimilation time:

$$
G^{2}\left(\tau_{a}\right)=\frac{1}{R^{2}}\left(1-e^{R \tau_{a}}\right)^{2}+\frac{F^{2}}{R^{4}}\left(1-e^{R \tau_{a}}\right)^{2}+e^{2 R \tau_{a}}
$$

The pseudo-inverse of the Hessian is given by

$$
\mathbf{H}^{+}\left(\tau_{a}\right)=\frac{\Delta u^{2}}{G^{4}}\left[\begin{array}{ccc}
\frac{1}{R^{2}}\left(1-e^{R \tau_{a}}\right)^{2} & -\frac{F}{R^{3}}\left(1-e^{R \tau_{a}}\right)^{2} & \frac{1}{R}\left(1-e^{R \tau_{a}}\right) e^{R \tau_{a}} \\
-\frac{F}{R^{3}}\left(1-e^{R \tau_{a}}\right)^{2} & \frac{F^{2}}{R^{4}}\left(1-e^{R \tau_{a}}\right)^{2} & -\frac{F}{R^{2}}\left(1-e^{R \tau_{a}}\right) e^{R \tau_{a}} \\
\frac{1}{R}\left(1-e^{R \tau_{a}}\right) e^{R \tau_{a}} & -\frac{F}{R^{2}}\left(1-e^{R \tau_{a}}\right) e^{R \tau_{a}} & e^{2 R \tau_{a}}
\end{array}\right]
$$

The profiles of time evolution of the Hessian pseudo-inverse terms are different and more complex than of the Hessian terms due to the nontrivial evolution of the normalizing 
factor. Figure 4.2 illustrates the nondimensionalized evolution of the resulting covariance terms labeled by the notation of (4.2.7). The variances of wind forcing and bottom friction coincide growing from zero reaching a peak and subsiding to their steady state value. The cross term of forcing-friction covariance is the negative mirror image, relaxing to the negative steady state covariance term of (4.1.15). We see that this negative constraint is not time invariant, immediately after model initialization the constraint is zero and it reaches a transient peak before stabilizing to the steady state. The variance of the initial conditions constraint starts from nonzero value, grows and decays to zero at the steady state, as was discussed in the previous section. The cross terms of forcing and friction covariances with the initial conditions have opposite signs and evolve from initial zero to zero value at the steady state, through a transient peak which can be very high and short in time, depending of the actual parameters of the problem. This analysis shows that cross coupling that involves initial conditions is transient in time, while the cross coupling of only friction and forcing parameters grows to a steady state value.

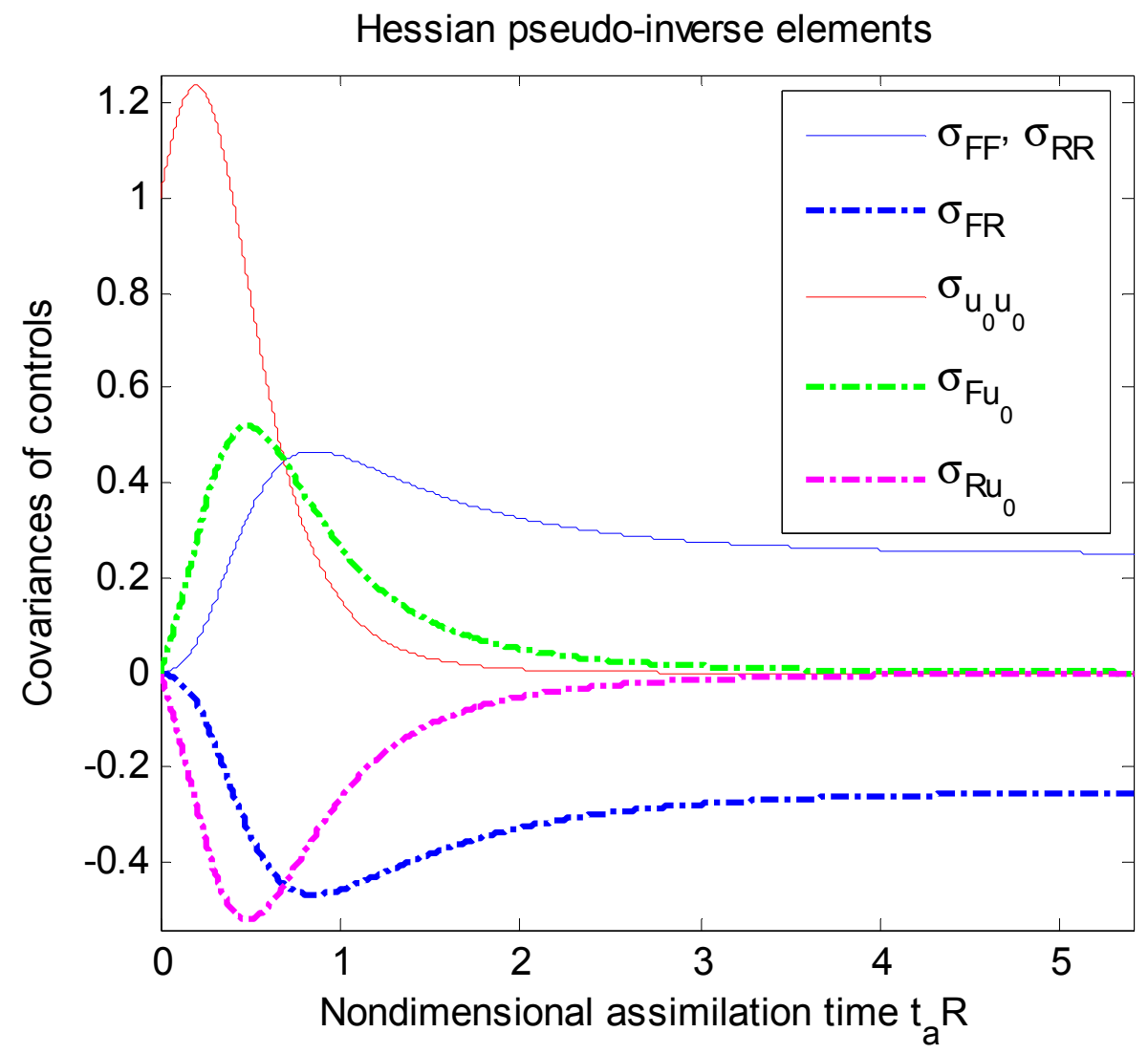

Figure 4.2 Evolution of nondimensionalized covariance terms given by the pseudo-inverse of the Hessian (4.2.15) as function of assimilation time. Each scalar term is labeled according to (4.2.7). 
It will be seen in Chapter 5 that most of the effect of uncertainty reduction of the ACC transport is achieved due to cross-coupling of controls. This uncertainty reduction is achieved when the cross terms of the quadratic product of the Jacobian vectors (4.2.6) with the posterior uncertainty reduction matrix (4.2.9) is positive. These terms of $\mathbf{g}^{T} \boldsymbol{\Gamma} \mathbf{g}$ can be positive either for positive or for negative covariance terms depending on the combination of signs of the Jacobian terms. Two examples are shown in Figure 4.3 which have been calculated analytically with our model. It is seen that cross coupling of friction and forcing acts to reduce uncertainty of the target transport in the steady state, growing gradually from zero. The cross coupling of forcing with initial conditions has only a transient effect, it grows faster but disappears at the steady state as the system evolves away from the initial conditions. Note, that the analytical expression of the target uncertainty reduction is an explicit function of two separate times - time of uncertainty assimilation and time of forward projection. In Figure 4.3 the abscissa shows the forward projection time, while the assimilation time is shown by the vertical line (dashed magenta) - a notation that will be used in Chapter 5.

Forward projected uncertainty reduction contribution for $t_{a}=0.25 / R$

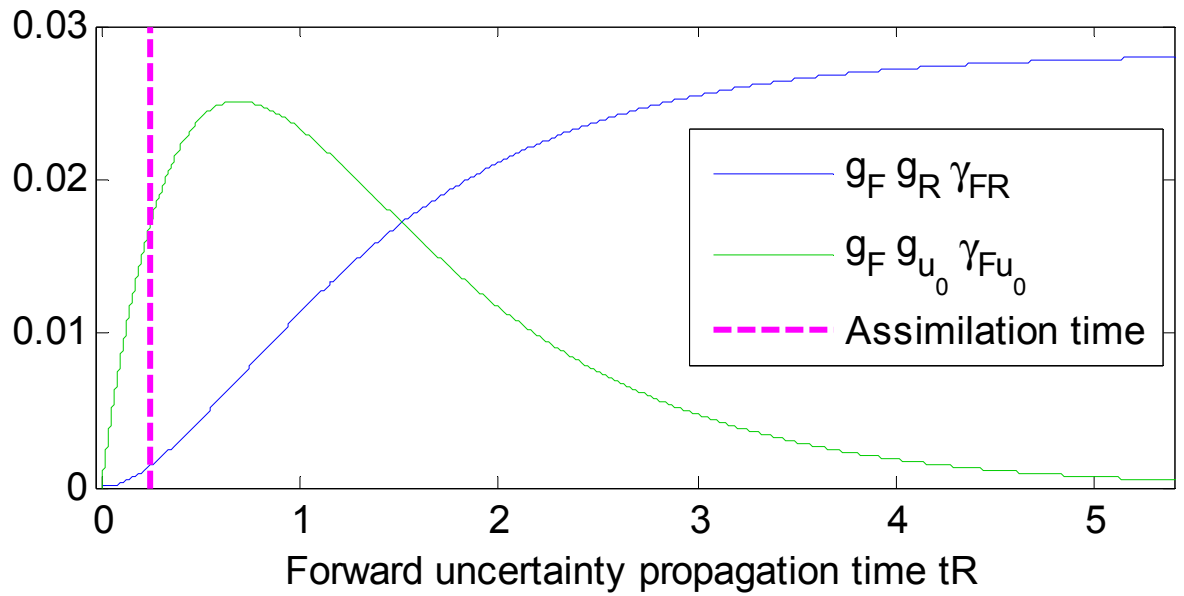

Figure 4.3 Evolution of nondimensionalized target uncertainty reduction terms - scalar contributions to sum $\mathbf{g}^{T} \boldsymbol{\Gamma} \mathbf{g}$, shown as function of forward uncertainty projection (abscissa) and uncertainty assimilation times (vertical dashed line). 


\section{UQ analysis with MITgcm model}

This chapter demonstrates application of the developed UQ methodology to a large-scale ocean state estimation problem. The UQ machinery was implemented in the MITgcm model of the ACC. Different synthetic observations, generated with the method of "identical twins" (Section 2.10), and their uncertainties are assimilated in the model. The data-constrained uncertainties of controls are mapped to uncertainties of the target variable - the Drake Passage transport. Two types of uncertainty calculations are implemented and analyzed: prior-independent uncertainty assimilation and prior-toposterior uncertainty reduction (see Section 2.9). The following sections illustrate and analyze the details of these calculations for different model configurations, different assimilated observations and different prior assumptions. The key building blocks of the methodology essential to understanding the uncertainty dynamics, the Jacobian fields, are discussed first in Section 5.1. Section 5.2 focuses on inverse uncertainty propagation. The prior-independent Hessian eigenvector fields and their eigenvalue spectra are illustrated in Section 5.2.1, the prior-to-posterior uncertainty reduction of controls is shown in Section 5.2.3. Forward uncertainty propagation experiments are examined in Section 5.3, comparing the dynamics of the prior and the posterior uncertainties of Drake Passage transport and analyzing the time-resolved evolution of uncertainty reduction. A schematic representation of the implemented UQ algorithms is shown in Figure 5.1, which also highlights the parts of the calculations visualized in the following sections.

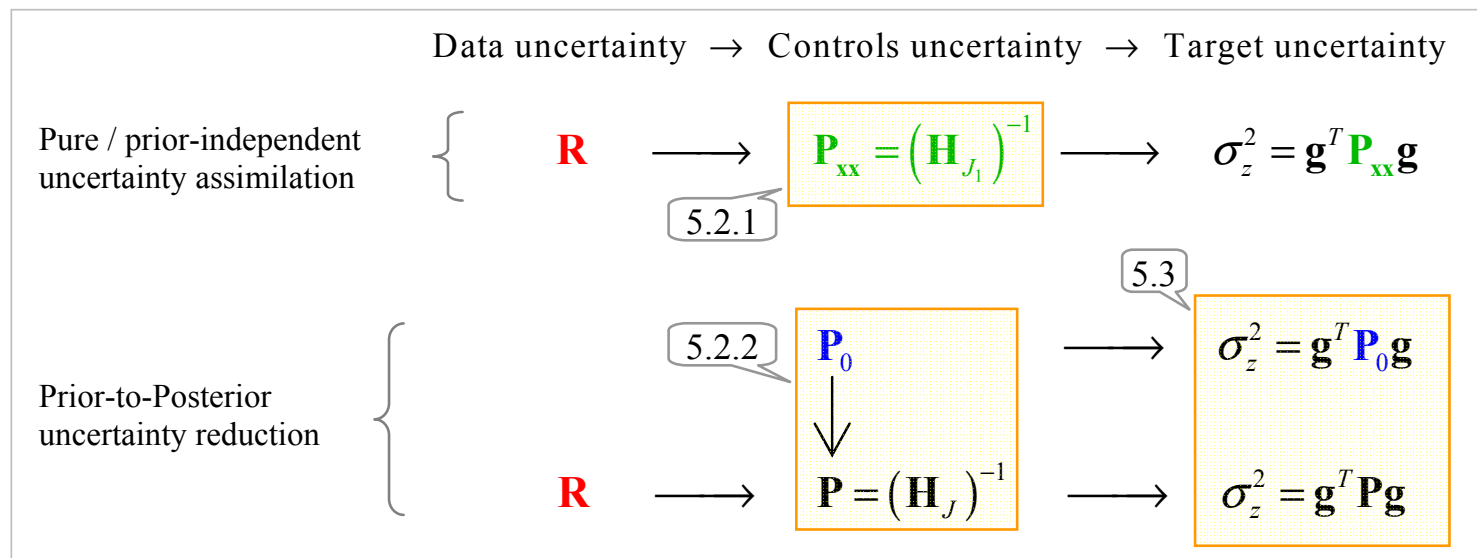

Figure 5.1 Comparison of the two different UQ schemes implemented in the MITgcm state estimation system. The rectangular shapes highlight the parts of the calculations visualized in the following sections, indicated by the numbers in the callouts. For more details on these UQ schemes see Section 2.9. 


\subsection{Sensitivity analysis}

As discussed in Chapter 4, understanding the structure of the model Jacobian fields explains the structure of the Hessian matrix in linear or zero residual misfit cases. Examination of these first derivative fields is also important in its own right and is known as sensitivity analysis (Heimbach et al. 2011). For the case of a single scalar model output, here - the Drake Passage transport, the Jacobian vector calculated with the adjoint model is known as the dual or the adjoint vector (Heimbach et al. 2011).

We show the adjoint (sensitivity) fields of the steady state circumpolar transport for the idealized and the realistic topography configurations of MITgcm, described in Chapter 3. In the following figures each 6 panel plot displays a single adjoint vector made of 6 twodimensional fields, each one of 80 by 180 scalar elements. The total size of the vector $80 * 180 * 6=86400$ elements. Each element is a partial derivative of the transport with respect to the perturbation of the corresponding element of one the six fields shown in the panels, i.e. each figure is equivalent to (4.2.12). The arrangement of the panels follows the scheme in Figure 5.2. The left side panels are sensitivities to forcing and friction fields, the right side panels are sensitivities to the initial conditions.

\begin{tabular}{|l|l|}
\hline Zonal wind stress $\tau^{\mathrm{x}}$ & Zonal flow $u_{t=0}$ \\
\hline Meridional wind stress $\tau^{\mathrm{y}}$ & Meridional flow $v_{t=0}$ \\
\hline Bottom drag coefficient $r$ & Surface elevation $\eta_{t=0}$ \\
\hline
\end{tabular}

Figure 5.2 Arrangement of control fields panels, all the 6 fields together constitute a single control vector.

This control vector is a generalization of the 3 element long control vector (4.1.3) and is intended to highlight the differences of adjoint forcing/dissipation vs. initial conditions fields.

Each of the figures shown below is the adjoint (sensitivity) vector for different adjoint time, defined as the backward time shift from the differentiated variable (transport) to the adjoint (4.2.5). The color scheme in the figures is symmetric with separate scale for each panel, shown by the colorbar to the right. The green color is zero sensitivity on all panels. As example, consider in detail Figure 5.3. The green zero sensitivity contour confines the regions of non-zero sensitivity around the idealized Drake Passage. Over the most of the ocean area the sensitivity is zero at adjoint time -2 hours, meaning that the transport at 
given time is not sensitive to any perturbation over this area two hour before. The sensitivity is not zero in the Passage area. It is positive for zonal wind forcing (upper left panel), meaning that a positive perturbation leads to increase of the transport 2 hours later. This sensitivity is not spatially homogeneous. It is maximum near the northern side of the Passage and gradually decreases to zero to the west and to the east of it. The sensitivity to the meridional wind stress changes sign across the Passage. It is negative to the west and in the Passage, but positive to the east of it. That means that a negative (southward) meridional stress perturbation to the west increases the transport, as well as a positive (northward) perturbation to the east. The sensitivity to the bottom friction (bottom left panel) is negative, meaning that increasing friction has negative effect on the transport. Note that the sensitivity to friction is zero over the southern part of the Passage. This is explained by realizing the nonlinear nature of the sensitivity concept, even that the effect of sensitivity is linear in the perturbation itself. The nonlinearity manifests by the fact that the sensitivity in general has a nontrivial dependence on system parameters. In the case of the sensitivity to the bottom friction, it is dependent on the magnitude of the flow itself, as is clear from the equations of motion (3.1.1). This is also evident in the explicit analytical form of sensitivity calculated for the ODE model (4.2.12). The sensitivity of the steady state circumpolar transport to bottom friction is stronger where the steady state flow is stronger. It is zero where there is no steady state flow, which can be confirmed on Figure 3.7.

Now consider the sensitivities of transport to the initial conditions two hours earlier, shown in the right panels in Figure 5.3. The sensitivity to the initial zonal flow (upper right panel) is positive to the west and much more so to the east of the Passage. It is in fact negative in the Passage itself. This highlights the effect of time shift between the differentiated variable (transport) and the adjoint, as well as the dynamics of the system as resolved by the evolving sensitivity fields. The sensitivity is propagating through the domain as it propagates backward in time and reflects the "adjoint dynamics". In this case the strong positive sensitivity propagating eastward and leaving behind the negative sensitivity in the Passage can be explained by fast Kelvin waves propagating westward along the Antarctic boundary, crossing $1000 \mathrm{~km}$ on the time scale of 1 hour (see Section 3.2). The positive sensitivity of the transport to zonal flow to the east of the Passage two 
hour earlier indicates that a positive zonal flow perturbation propagates from that area on the east to the west into the Passage reaching it exactly in two hours time. Note, that the resulting "adjoint Kelvin waves" propagate in the opposite direction of the actual Kelvin waves, carrying the sensitivity information backward in time. Such "adjoint waves" are also known as the "dual waves" (Heimbach et al. 2011) and can be regarded as "sensitivity waves" as well. In the context of uncertainty quantification, similar waves in the uncertainty fields which propagate the uncertainty information across the domain will be regarded as "uncertainty waves". Back to the explanation of the zonal flow sensitivity map, the positive sensitivity to the west of the Passage can also be explained by the adjoint Kelvin waves, but those propagating westward and northward around the southwestern corner of the rectangular barrier representing the American continent. These west-northward adjoint Kelvin waves are also seen in the meridional flow sensitivity map (Figure 5.3 middle right panel) as a strong negative sensitivity around the barrier southwest corner. The fast time scale adjoint Kelvin waves are also seen in the sensitivity to the initial surface elevation (Figure 5.3 bottom right panel).

\subsubsection{Aqua planet with barrier}

Now we overview the sensitivity results for the idealized aqua planet with barrier (Figure 5.3 to Figure 5.8). The change of sensitivity fields from time to time is very substantial and highlights the importance of understanding of time evolution of the adjoint fields. All sensitivities appear first in the local area around the Drake Passage and spread all over the planet in the first 24 hours. The sensitivities to initial conditions decay to zero with adjoint time, while the sensitivities to forcing and friction grow to steady state values. The sensitivities exponentially reach steady state after 60 to 90 days. The convergence was confirmed by running the adjoint model for 360 days (Figure 5.8). Both the growth and the decay of sensitivities were predicted by the analytical solution (4.2.12). Note, that the adjoint integration starts from a steady state of the forward model and thus, as predicted by the analytical model, we do not resolve some possibly peculiar transient sensitivities (see the discussion in Section 4.2).

In more detail, Figure 5.3 shows that the sensitivity to steady zonal wind forcing is positive and grows from $\mathrm{O}\left(10^{-1} \mathrm{~Sv} / \mathrm{Pa}\right)$ to $\mathrm{O}(5 \mathrm{~Sv} / \mathrm{Pa})$ at the steady state (Figure 5.7). The 
steady state sensitivity is confined to the southern hemisphere inside a zonal band, wider than the applied wind stress jet. However, during the transient adjustment of the sensitivity there is a significant global contribution peaking at the equatorial latitudes to the west of the barrier (Figure 5.5). The maximum sensitivity is always just next to the northern side of the Drake Passage. The transient sensitivity to the meridional wind stress is negative to the west and positive to the east of the Passage, with maxima located just to the north of the latitude of the Passage. The meaning is that south blowing winds to the west of the barrier force the flow into the Passage and north blowing winds to the east of the barrier accelerate the transport by pushing the water out of the Passage. During the transient sensitivity growth the western negative sensitivities grow and spread along the western coast of the barrier (Figure 5.5), while the eastern positive sensitivities remain small and confined. At the steady state only a narrow region remains sensitive around the southern tip of the barrier, the negative sensitivity on the west side is strong $\mathrm{O}(-5 \mathrm{~Sv} / \mathrm{Pa})$ (Figure 5.7). The sensitivity to bottom friction coefficient spreads zonally from the Passage to circumscribe the planet at the latitude band of the applied wind forcing and the resulting flow jet. The sensitivity is negative everywhere and peaks near the southern coast of the barrier, where it grows from $-2 \cdot 10^{6} \mathrm{~m}^{2}$ to $-9 \cdot 10^{7} \mathrm{~m}^{2}$. The sensitivity to the initial velocities decays from the initial values of $\mathrm{O}\left(5 \cdot 10^{7} \mathrm{~m}^{2}\right)$ after 2 adjoint hours to $\mathrm{O}\left(2 \cdot 10^{5} \mathrm{~m}^{2}\right)$ after 90 days to $\mathrm{O}\left(4 \cdot 10^{-1} \mathrm{~m}^{2}\right)$ after 360 days. We note that, the area of the vertical western face of a single grid cell is $1.1 \cdot 10^{9} \mathrm{~m}^{2}$, which is equal to the adjoint of the transport to zonal flow at adjoint time 0, i.e. before any effects of the dynamic adjoint model. The sensitivity to the initial surface elevation decays from absolute values of $\mathrm{O}\left(2 \cdot 10^{6} \mathrm{~m}^{2} \mathrm{~s}^{-1}\right)$ after 2 hours of adjoint integration. The sensitivities to the initial conditions include positive and negative values and in the transient stage exhibit strong global and equatorial signatures (Figure 5.5), as well as wavy structures propagating along the equator from west to east (Figure 5.6). The latter can be referred to as sensitivity Rossby waves and they are an explicit manifestation of oceanic teleconnections as resolved by the adjoint model. Their mechanism can be explained as the adjoint of equatorial Rossby waves, which propagate from east to west on time scale of 3 days and longer for shorter wavelengths (see Section 3.2). 


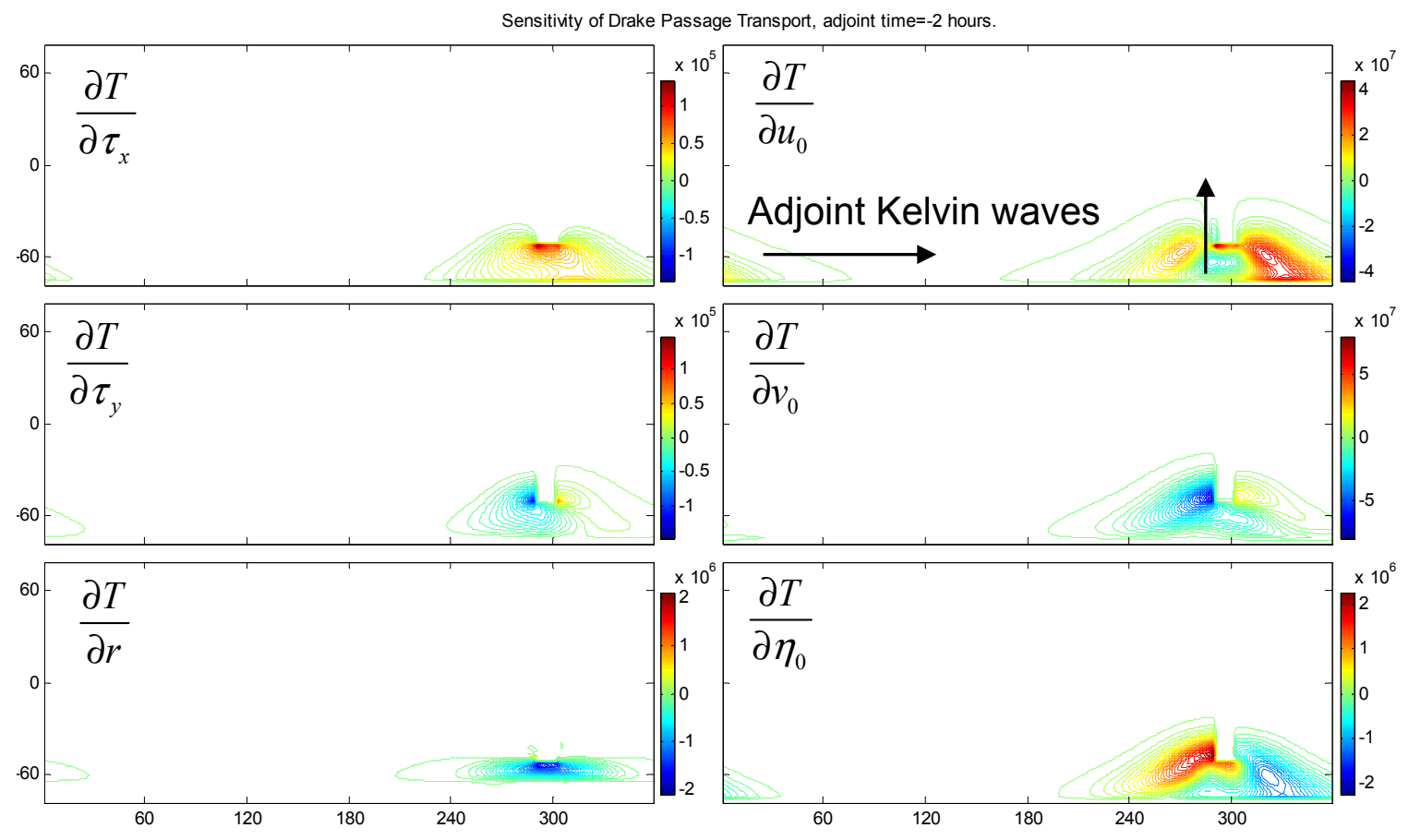

Figure 5.3 Adjoint (sensitivity) vector of Drake Passage transport to (columnwise top to bottom and left to right): zonal wind stress $\left(\mathrm{m}^{3} \mathrm{~s}^{-1} \mathrm{~Pa}^{-1}\right)$, meridional wind stress $\left(\mathrm{m}^{3} \mathrm{~s}^{-1} \mathrm{~Pa}^{-1}\right)$, bottom friction coefficient $\left(\mathrm{m}^{2}\right)$, initial zonal velocity $\left(\mathrm{m}^{2}\right)$, initial meridional velocity $\left(\mathrm{m}^{2}\right)$, and initial surface elevation $\left(\mathrm{m}^{2} \mathrm{~s}^{-1}\right)$. Shown for aqua planet with barrier configuration at adjoint time -2 hours.

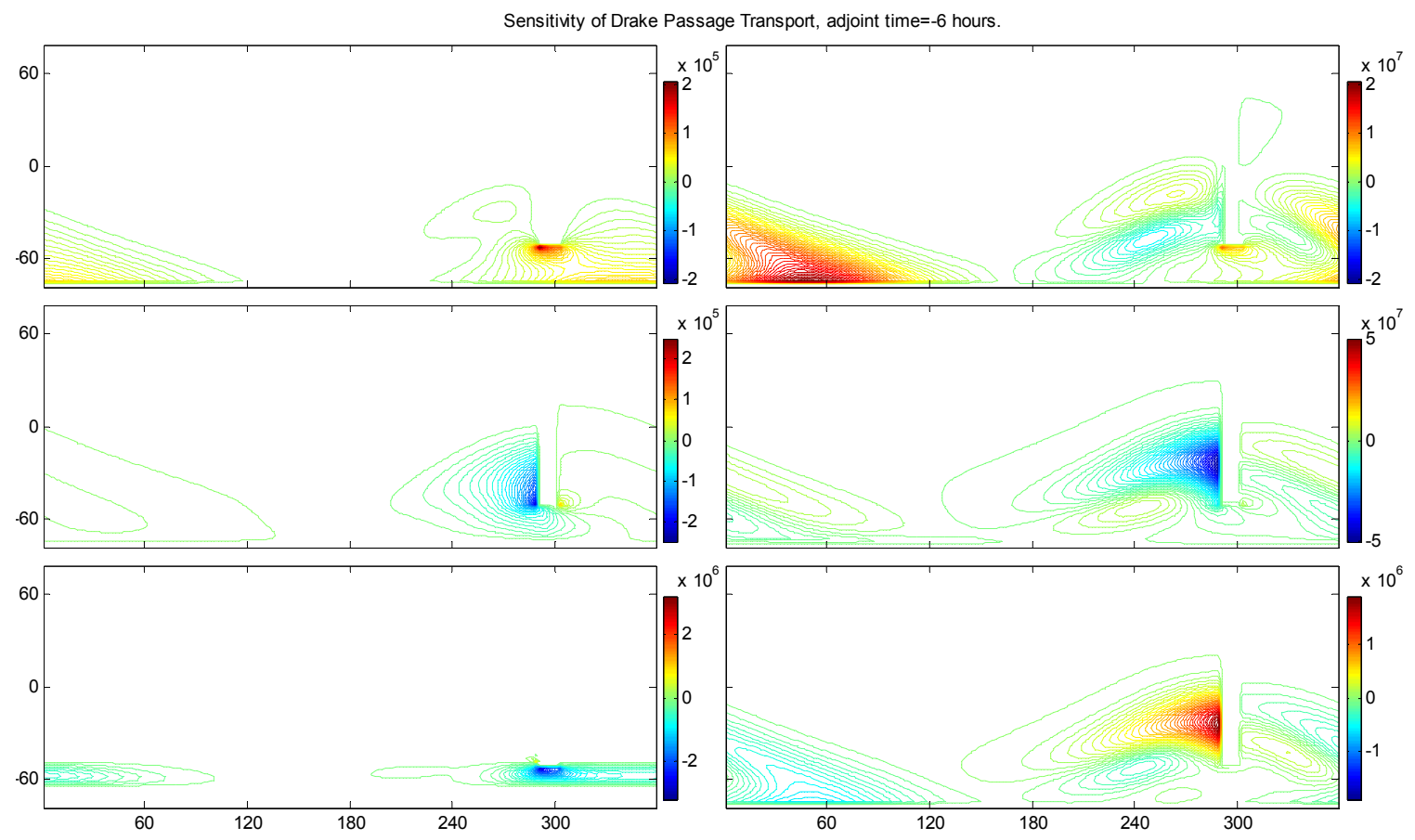

Figure 5.4 Same as in Figure 5.3. Shown for aqua planet with barrier configuration at adjoint time -6 hours. 


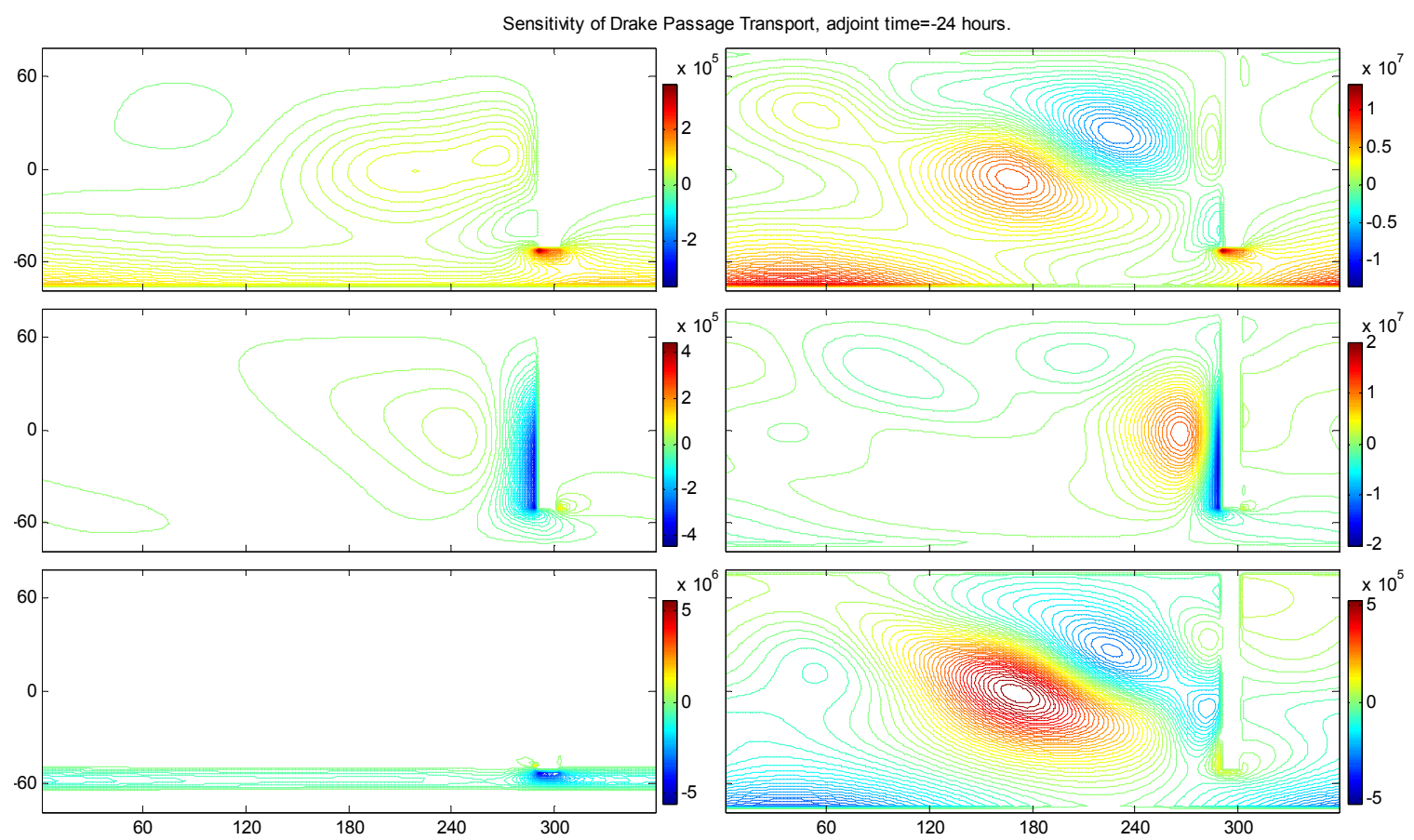

Figure 5.5 Same as in Figure 5.3. Shown for aqua planet with barrier configuration at adjoint time -24 hours.

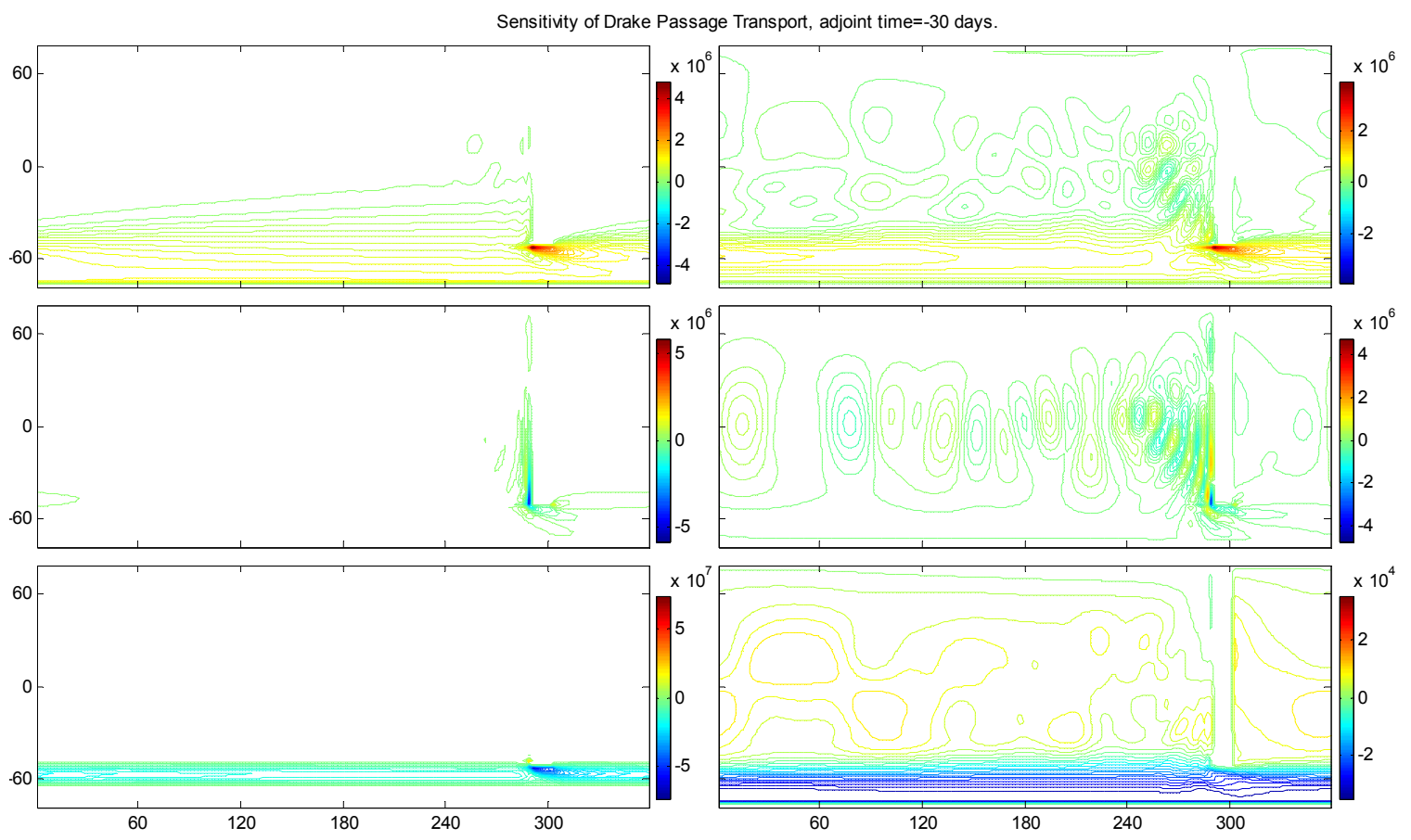

Figure 5.6 Same as in Figure 5.3. Shown for aqua planet with barrier configuration at adjoint time -30 days. 


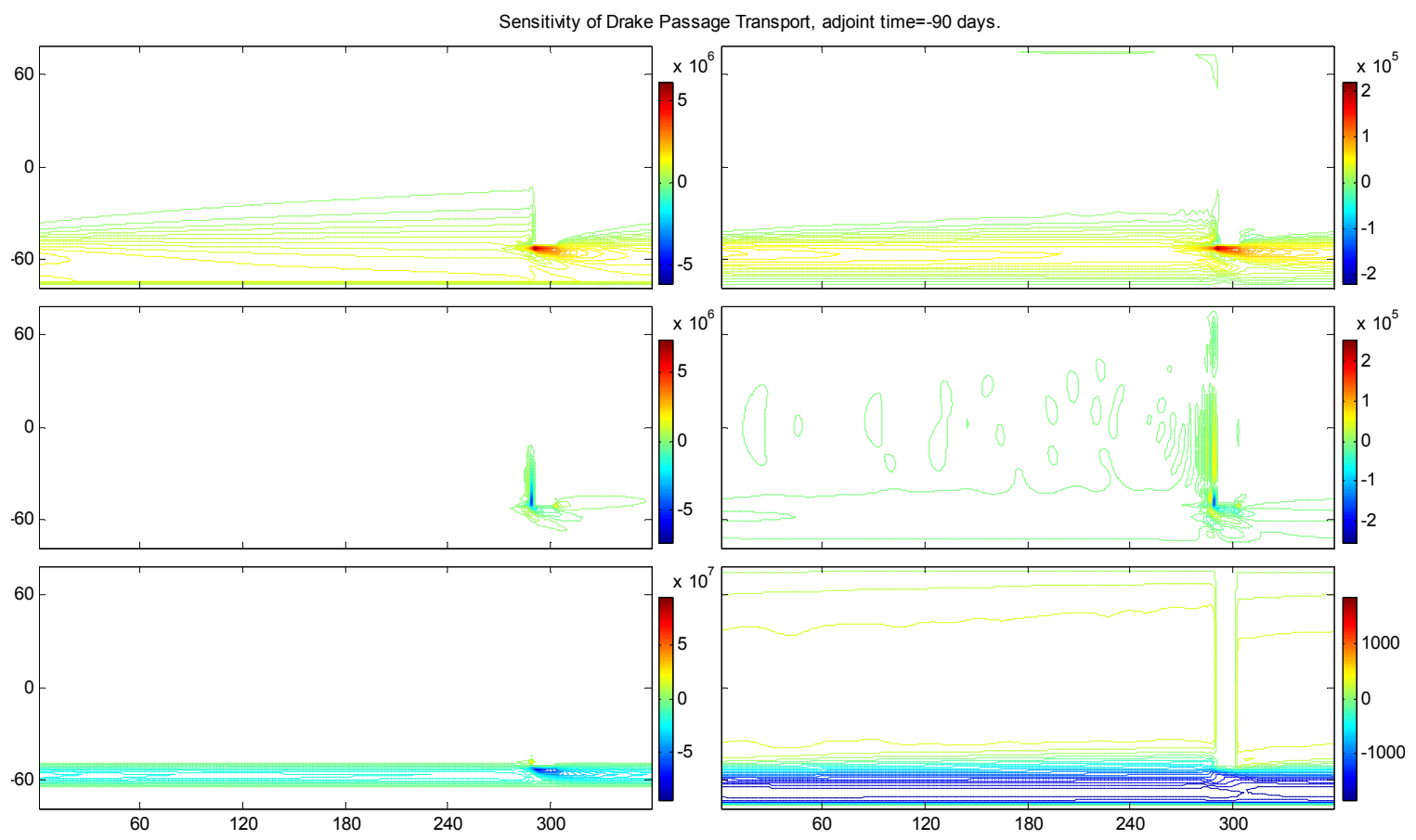

Figure 5.7 Same as in Figure 5.3. Shown for aqua planet with barrier configuration at adjoint time -90 days approximating convergence to steady state sensitivity.

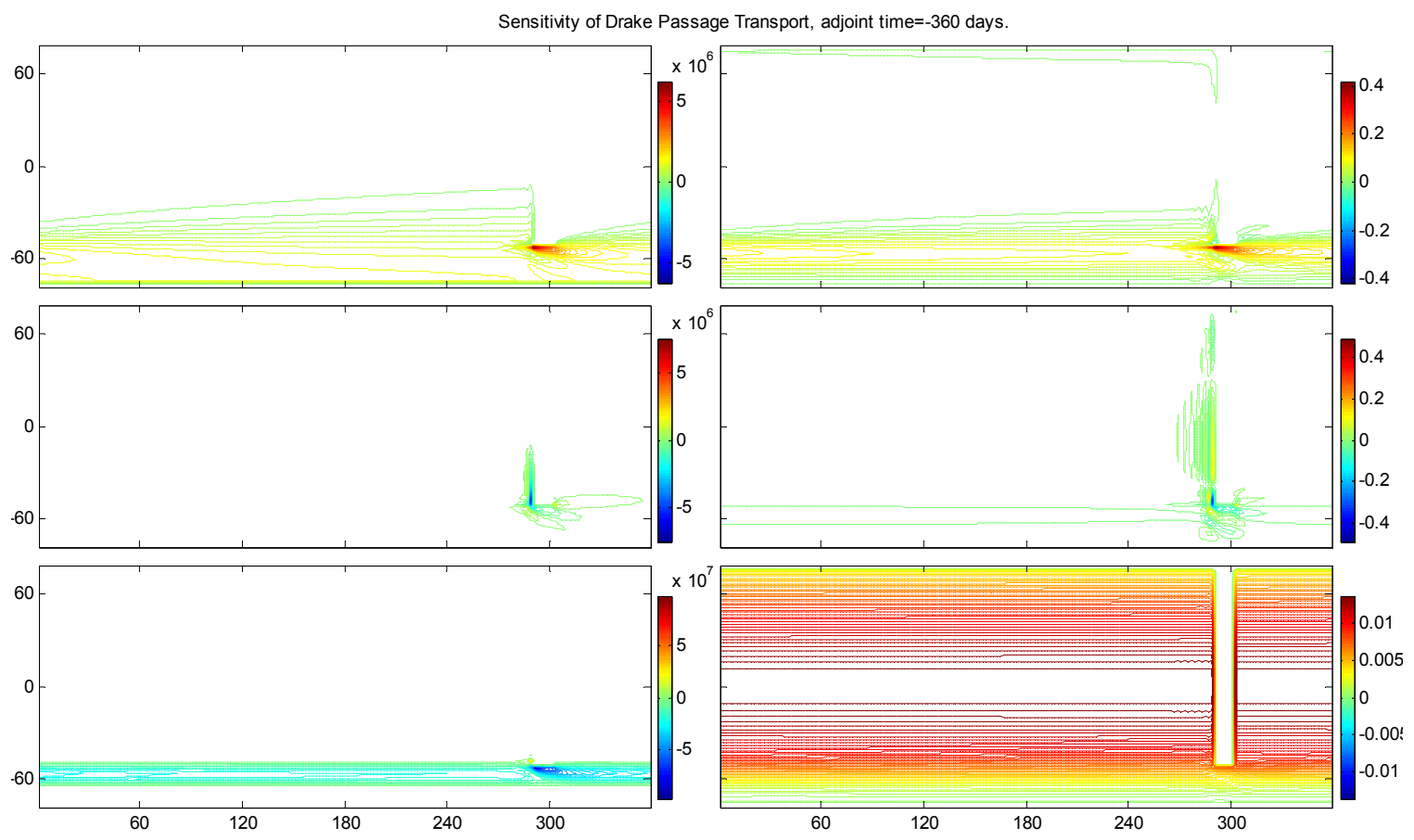

Figure 5.8 Same as in Figure 5.3. Shown for aqua planet with barrier configuration at adjoint time -360 days approximating convergence to steady state sensitivity. 


\subsubsection{Realistic geometry with flat bottom}

Next we examine the sensitivity fields for the realistic geometry with flat bottom configuration. Qualitatively the dynamics of sensitivity is very similar to the aqua planet with barrier case. All sensitivities appear in the first hours only around the Drake Passage area and spread over the planet by the end of the first day. The sensitivities to forcing and friction grow to their steady state patterns, while the initial conditions sensitivities decay. The positive sensitivity to zonal wind stress grows from $\mathrm{O}\left(10^{-1} \mathrm{~Sv} / \mathrm{Pa}\right)$ to $\mathrm{O}(5 \mathrm{~Sv} / \mathrm{Pa})$, while the negative sensitivities to the meridional stress on the west of the Passage start from slightly weaker magnitudes. The transient sensitivity to southward winds is very prominent along the western coasts of both American continents (Figure 5.11). The sensitivity to bottom friction is negative, as expected, as the band of sensitivity encircles the globe following the pattern of the flow. The magnitude is higher, growing from $-5 \cdot 10^{6}$ $\mathrm{m}^{2}$ to $-15 \cdot 10^{7} \mathrm{~m}^{2}$, which can be explained by the narrower Passage geometry. The evolution of sensitivities to the initial conditions is qualitatively and quantitatively similar to the previous examined case, with strong transient teleconnections signatures and west to east propagating waves. The differences in the specific patterns can be attributed to the more complex geometry, which for example capture the effect of the circulation around the Australian continent.

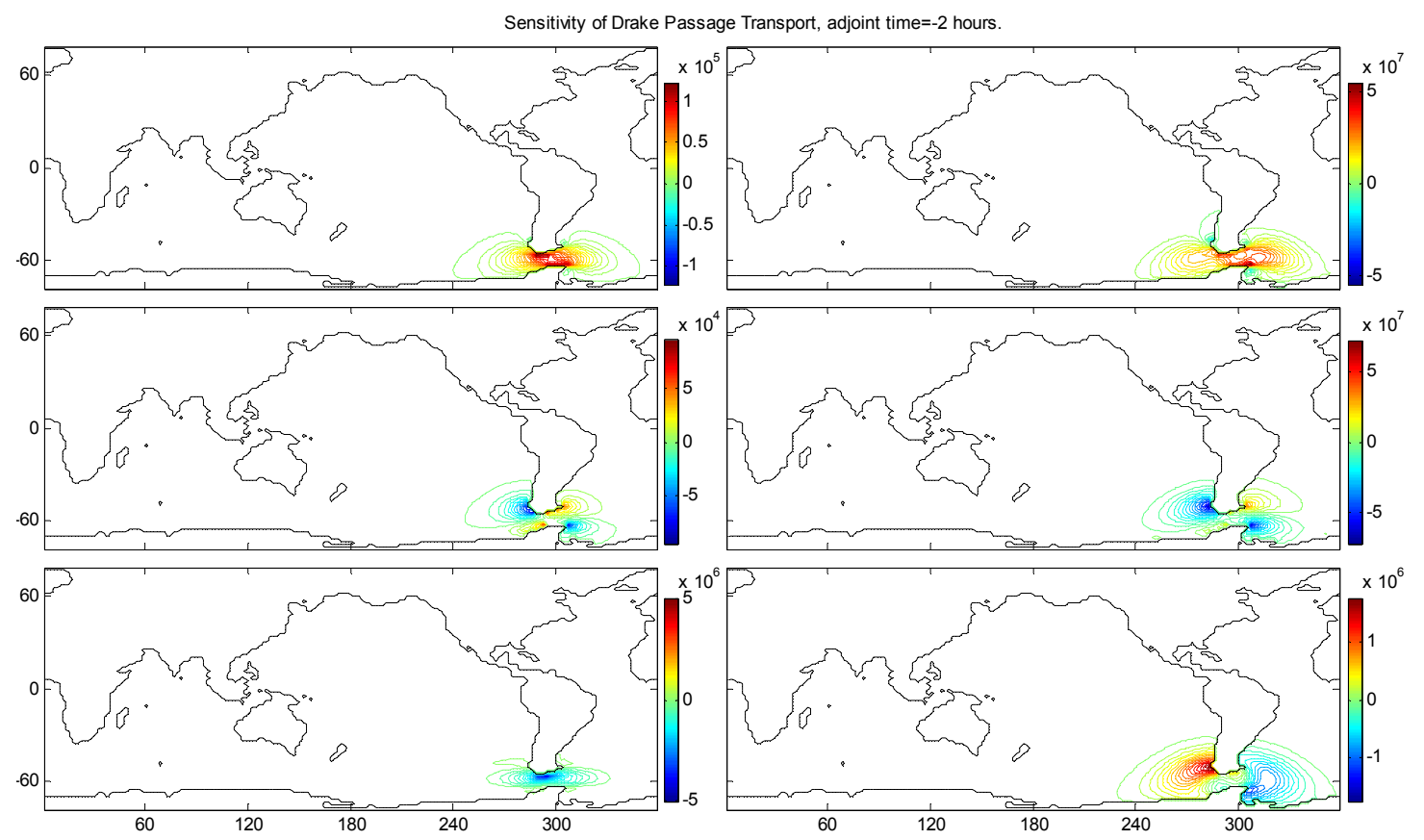

Figure 5.9 Same panels as in Figure 5.3 with additional black contour outlining the coastline. Shown for realistic geometry with flat bottom configuration at adjoint time -2 hours. 


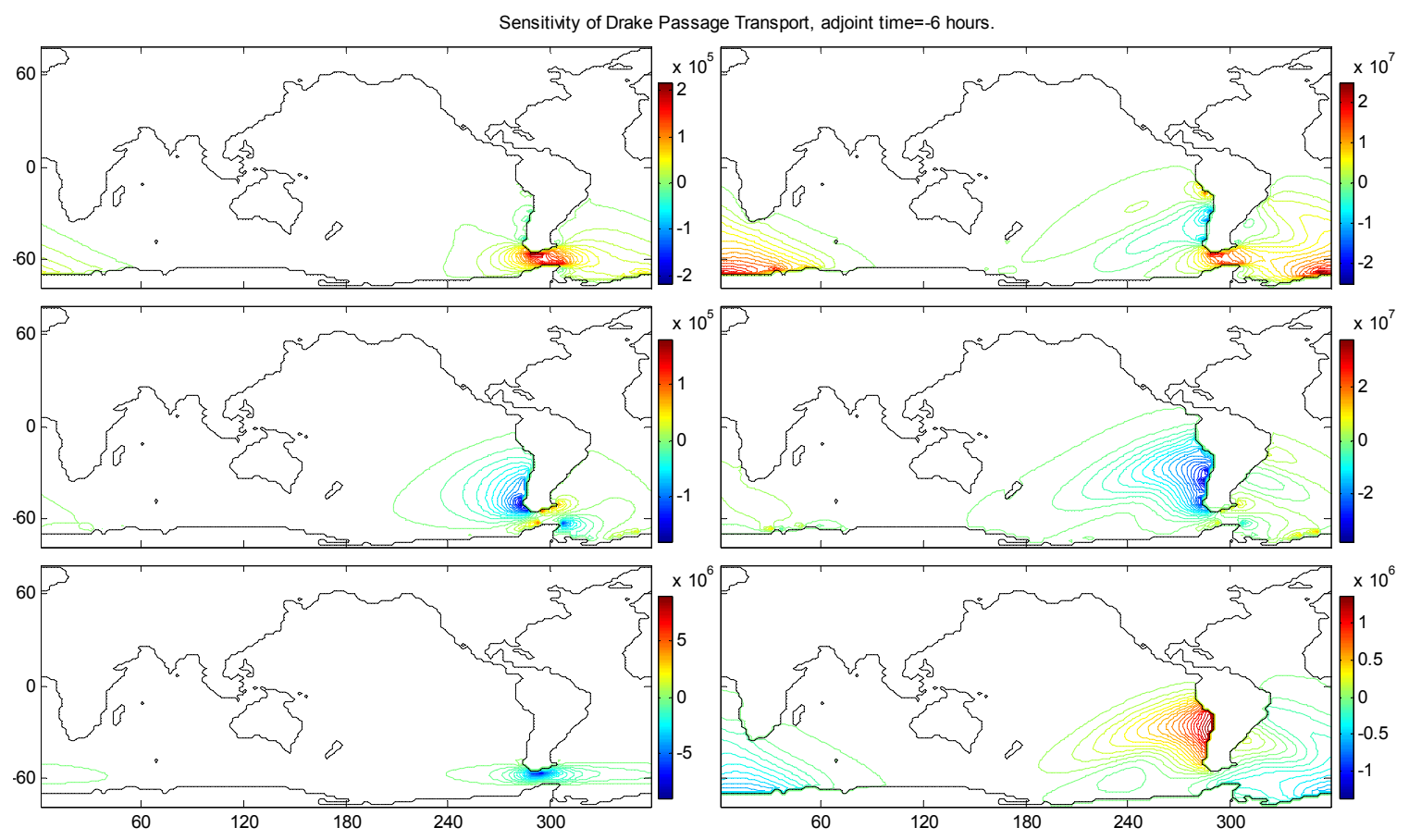

Figure 5.10 Same panels as in Figure 5.3 with additional black contour outlining the coastline. Shown for realistic geometry with flat bottom configuration at adjoint time -6 hours.

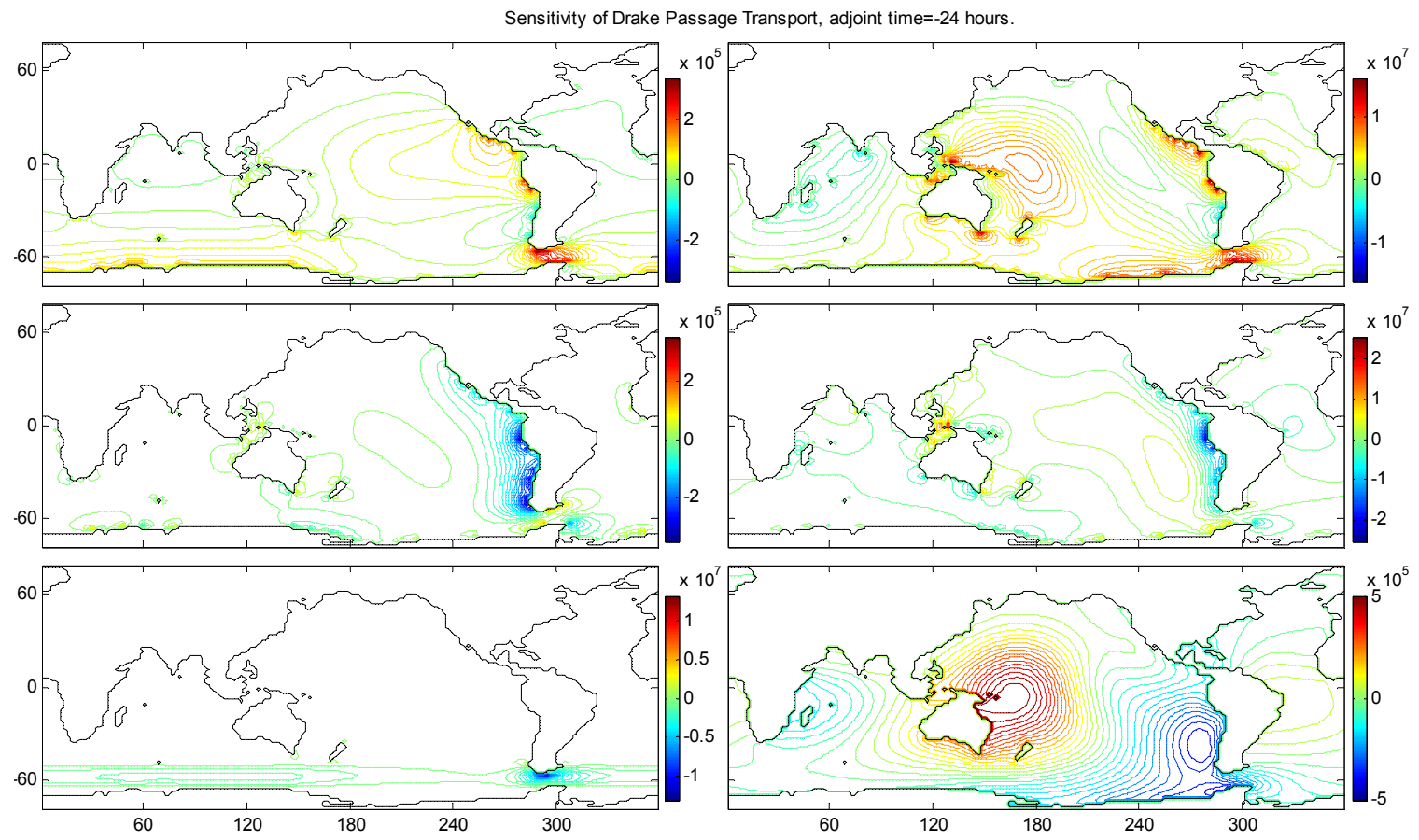

Figure 5.11 Same panels as in Figure 5.3 with additional black contour outlining the coastline. Shown for realistic geometry with flat bottom configuration at adjoint time -24 hours. 


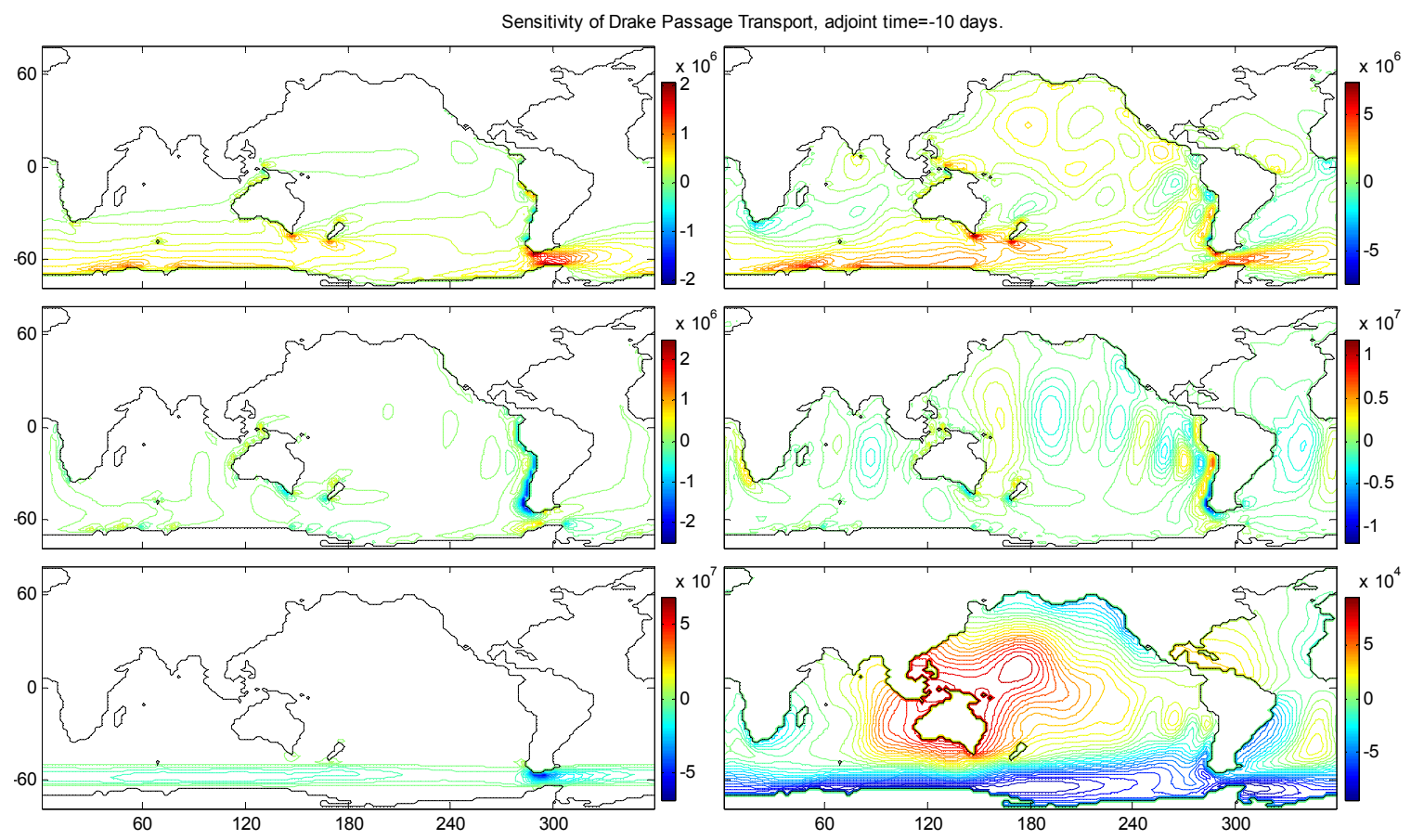

Figure 5.12 Same panels as in Figure 5.3 with additional black contour outlining the coastline. Shown for realistic geometry with flat bottom configuration at adjoint time -10 days.
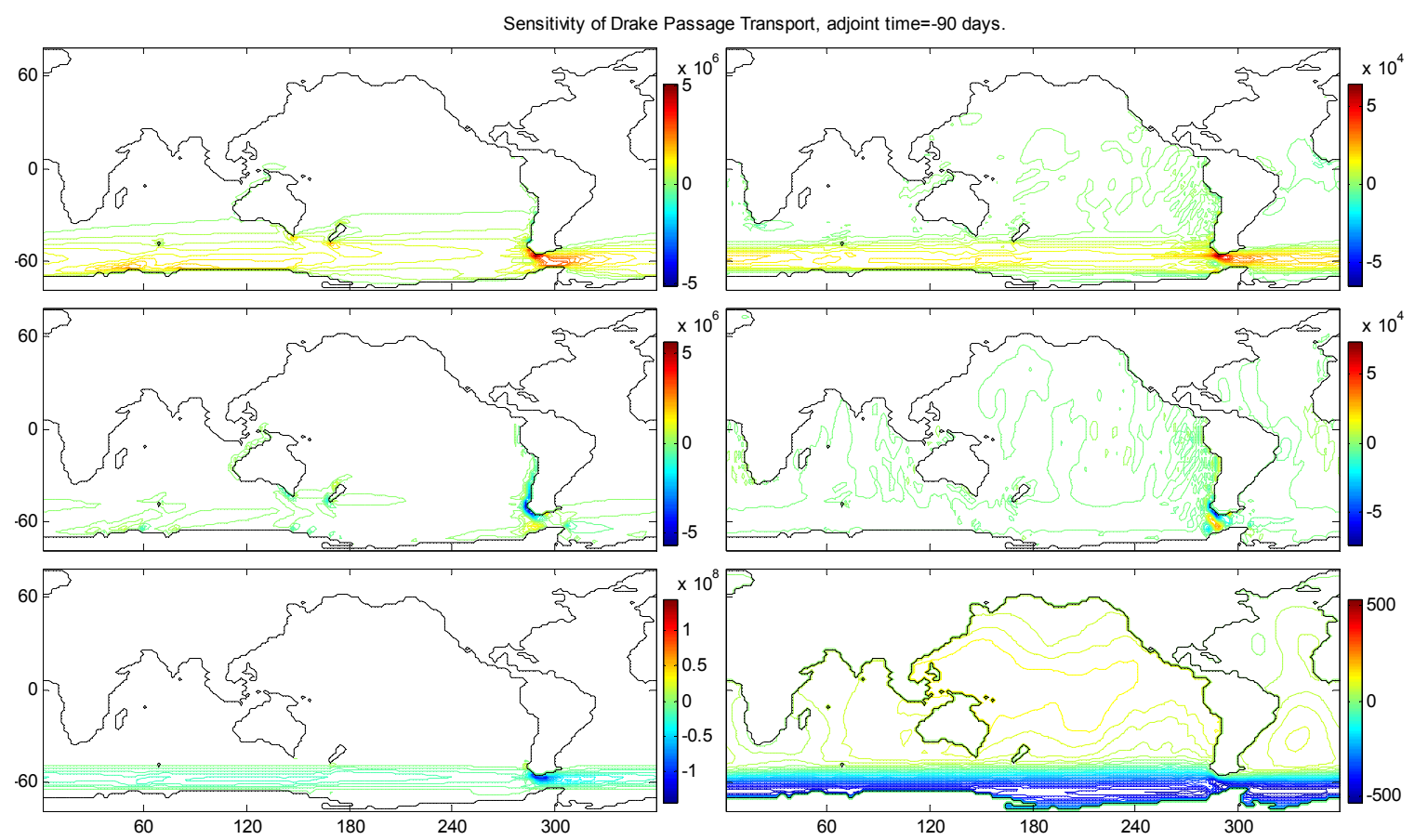

Figure 5.13 Same panels as in Figure 5.3 with additional black contour outlining the coastline. Shown for realistic geometry with flat bottom configuration at adjoint time -90 days approximating convergence to steady state sensitivity. 


\subsubsection{Realistic bathymetry}

The sensitivities of the transport in the realistic bathymetry case are markedly different from the flat bottom configurations, indicating that the bottom topography or geometry play a critical role in the dynamics of the system response to wind forcing. The patterns of the sensitivity fields appear to follow the topography exhibiting a finer spatial scale. One of the leading order explanations might be the consequence of the flow itself being governed by the topography. The flow aligns with the geostrophic contours due to conservation of the potential vorticity and so are the localized sensitivity effects. This is in contrast with remote sensitivity effects due to ocean teleconnections. The sensitivity to bottom friction appears to follow closely the contours, but unlike in the flat bottom case there are regions of strong positive sensitivity along the path of the ACC. This appears counterintuitive that strengthening of the bottom friction may increase the overall transport, but may be explained if in these sensitive "choke points" the increased resistance to the flow pushes the current to an alternative path with overall less resistance. Unlike in the flat bottom cases, the sensitivity to zonal wind forcing is not confined to one zonal band and splits to two main regions - along the path of the ACC and along the equator. The meridional stress sensitivity is not confined only to the narrow areas along the continents as before, but appears also along most of the path of the ACC. Nonetheless, the sensitivity along the coasts remains significant. The sensitivity to the initial conditions still decays to zero but the patterns appear more fragmented. The transient teleconnections and eastward waves are still noticeable, however a different pattern of longitudinal standing waves dominates (Figure 5.14). In the later adjoint times (Figure 5.15), as the steady state settles and the initial conditions sensitivities are small, the equatorial areas exhibit unusual patterns of sensitivity that may be related the numerics of the C-D scheme used and would require a higher resolution simulation to understand if these are not just numerical artifacts. 

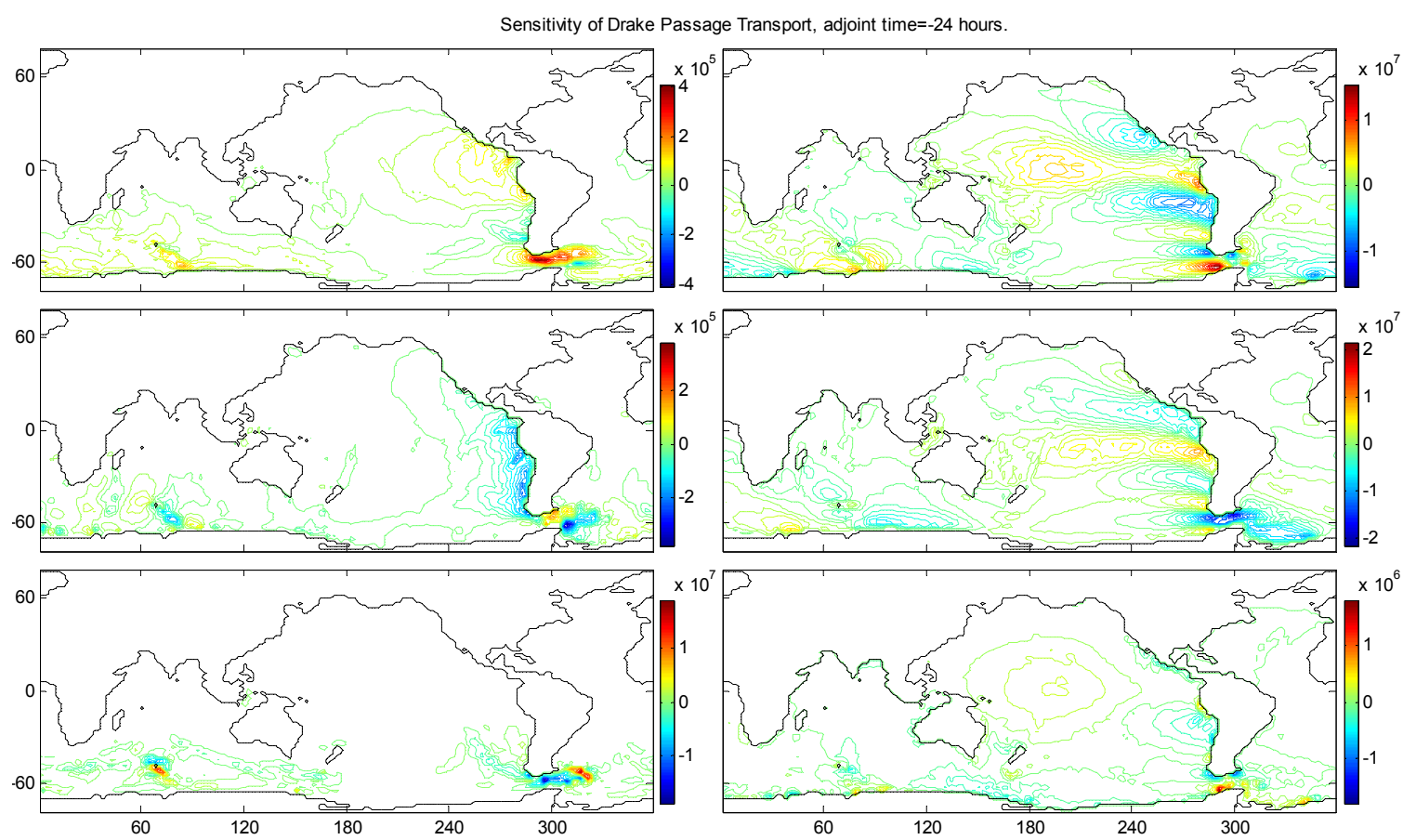

Figure 5.14 Same panels as in Figure 5.3 with additional black contour outlining the coastline. Shown for realistic bathymetry configuration at adjoint time -24 hours.
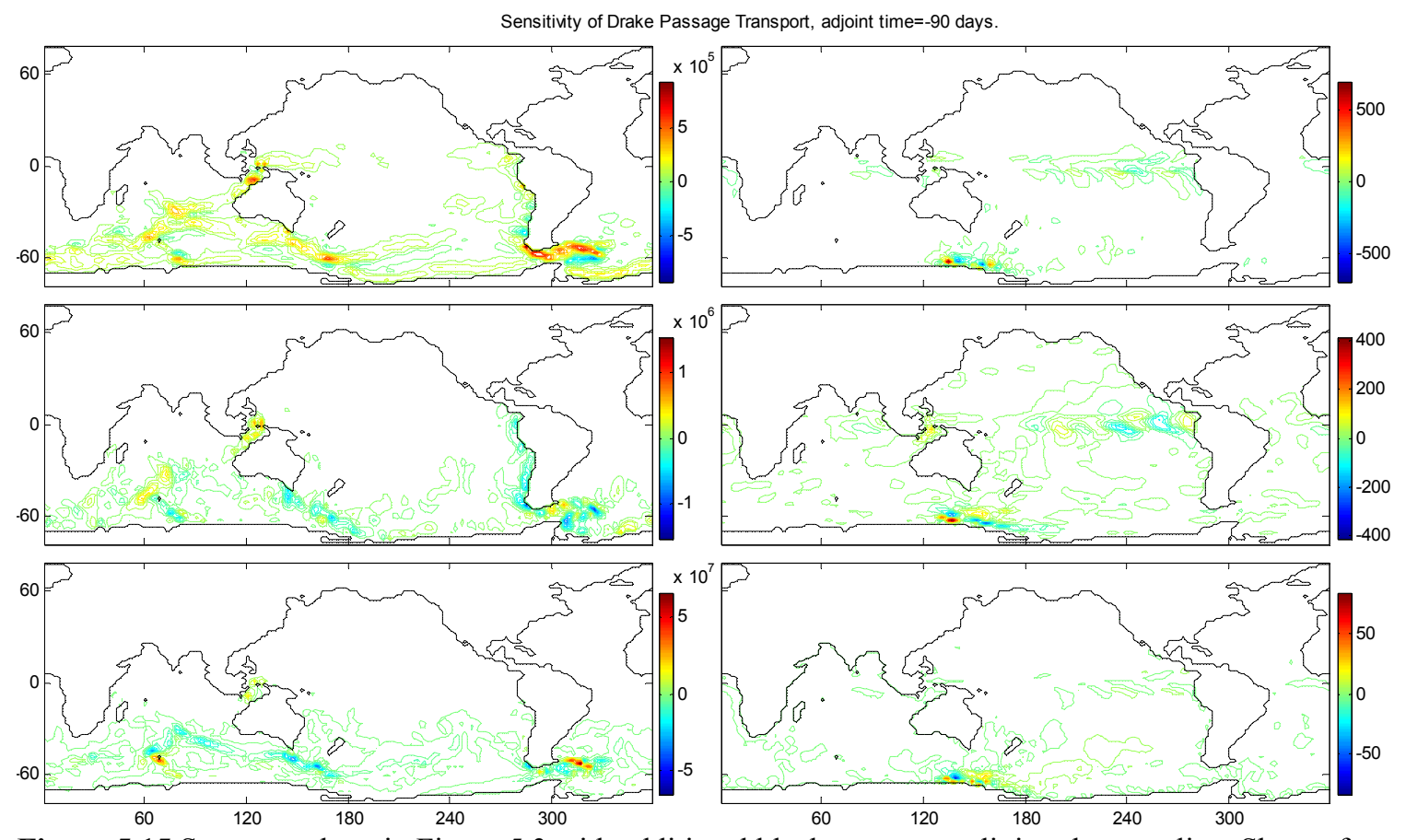

Figure 5.15 Same panels as in Figure 5.3 with additional black contour outlining the coastline. Shown for realistic bathymetry configuration at adjoint time -90 days, approximating the steady state sensitivities. 


\subsubsection{Time scales and evolution of sensitivity}

In this section we analyze in detail the time scales and evolution of the resolved dynamics of sensitivity fields. The magnitudes of sensitivity fields can be quantified by separate Frobenius norms of each two-dimensional adjoint fields. The resolved dynamics of the spatially integrated MITgem sensitivities are very similar to the exponential evolution in the analytical model (4.2.12). The sensitivities to wind forcing and bottom friction grow with adjoint time, exponentially approaching the steady state values (Figure 5.16). The sensitivities to the initial conditions exponentially decay (Figure 5.17).

A detailed analysis of the sensitivity evolution shows that one time scale dominates the dynamics of the system. After initial 4-day adjustment, characterized by oscillations with time scale of 1 day, the systems enters an exponential regime. The exponential regime prevails for 300 days, controlling the system on synoptic barotropic time scales. Figure 5.17 quantifies the details of the spin down of initial conditions sensitivity. The dominant slow exponential relaxation time scale is exactly 20.5 days for both surface elevation and currents initial conditions. It is consistent with the frictional spin down time scale $O(12$ days) but is roughly a factor two slower. The fast initial transient decay can be approximated by exponential relaxation with time scale of roughly 3.3 days for current and 4.9 days for surface elevation initial conditions sensitivities. This fast relaxation and the transient oscillations must be associated with the fast geostrophic adjustment by Kelvin and inertia-gravity waves.
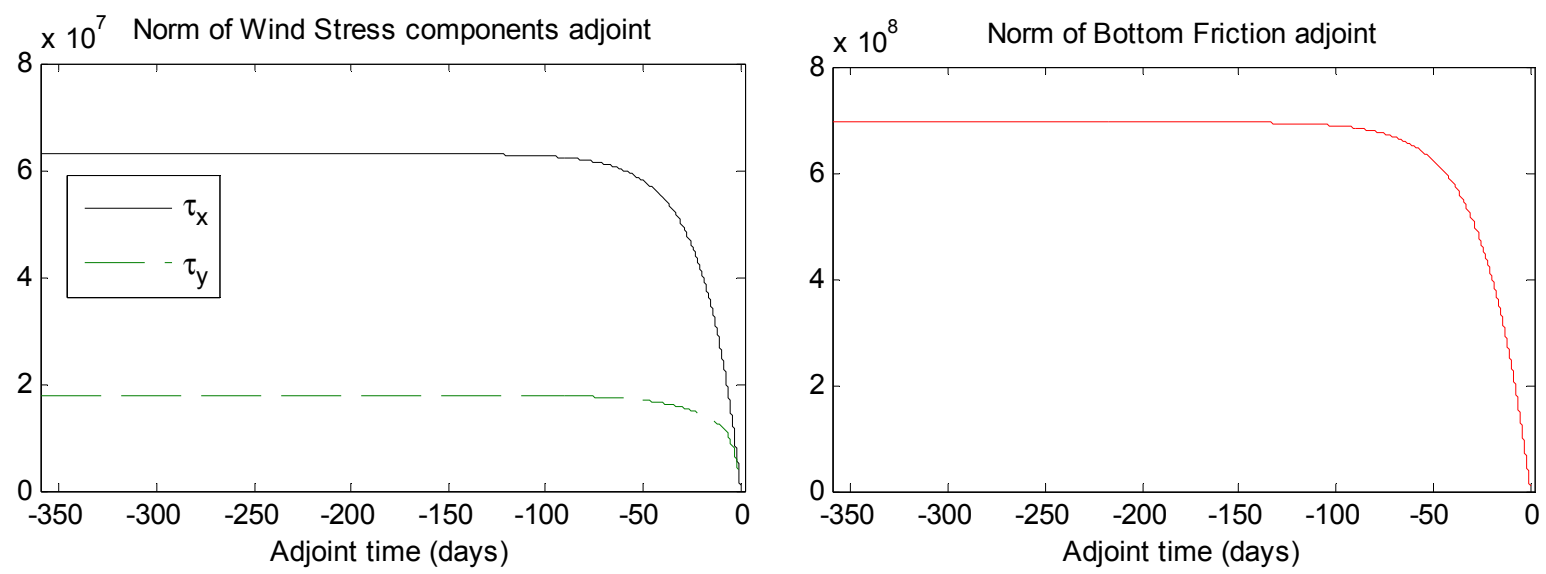

Figure 5.16 Frobenius norms of wind stress components and bottom friction coefficient sensitivities. Shown for the idealized aqua planet with barrier sensitivity calculations. 
On long time scale, after 320 days the norm of initial surface elevation exhibits another transition to even slower decay. That transition may be associated with the eddy viscosity dissipation acting on the diffusive time scale of $O(300$ days), see discussion in Section 3.2 .
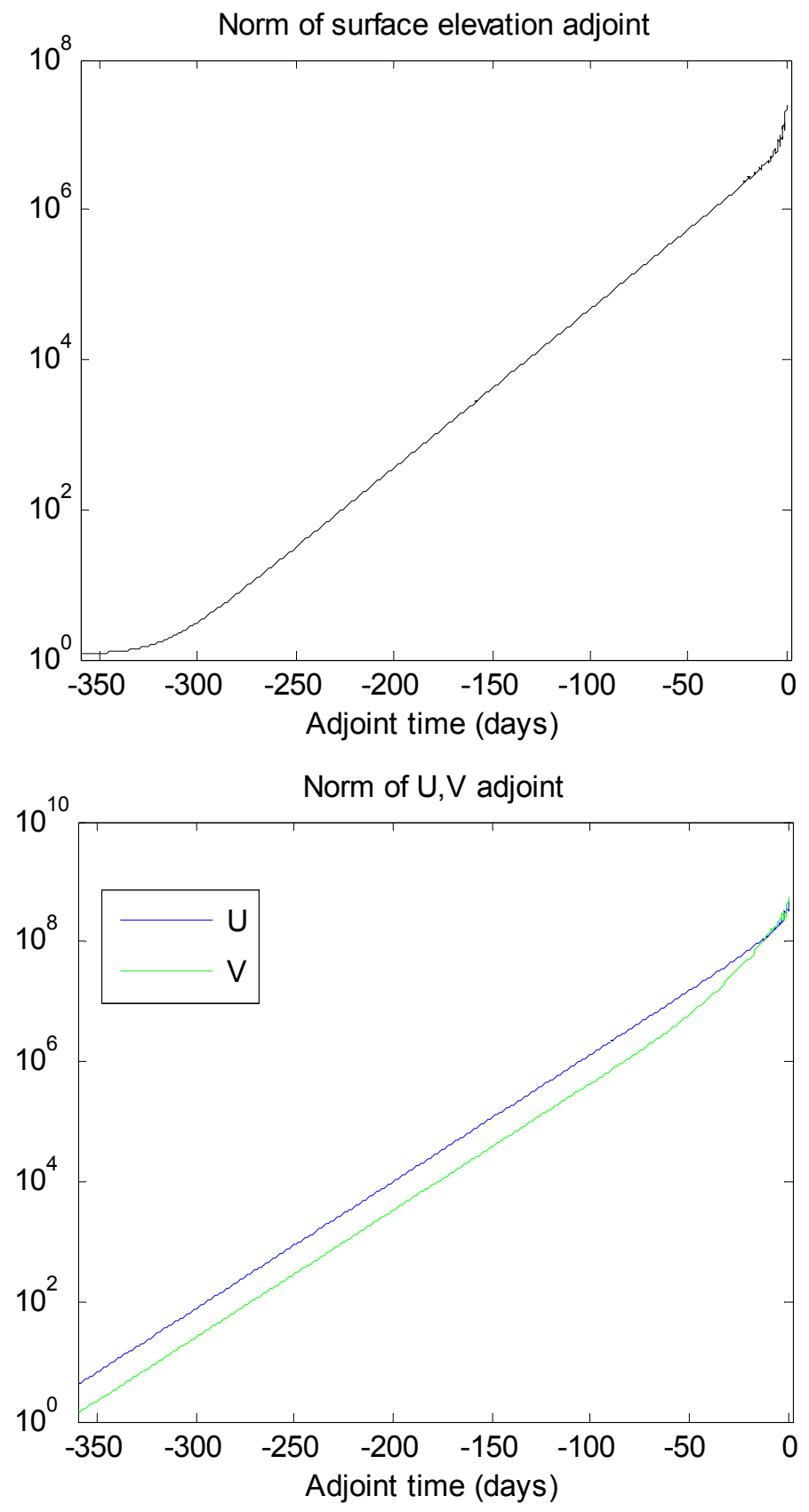

Figure 5.17 Frobenius norms of surface elevation (upper panel) and velocity components (lower panel) adjoint fields as function of adjoint time. Two exponential fits are shown for each - surface elevation and the zonal velocity component. Shown for the idealized aqua planet with barrier sensitivity calculations. 


\subsection{Inverse uncertainty propagation}

In this inverse uncertainty propagation section we analyze the details of uncertainty assimilation in MITgcm. We first introduce the different synthetic observation systems designed for the uncertainty assimilation experiments, sub-section 5.2.1. Then, in subsection 5.2.2, we analyze the unbiased prior-independent uncertainty assimilation by exploring the principal uncertainty patterns resolved by the Hessian eigenvectors. We examine the spectra of the Hessians, which properties are key to the efficiency of our numerical implementation of the UQ methodology. The last sub-section (5.2.3.) of this inverse uncertainty propagation section combines the Hessians with prior uncertainties and focuses on prior-to-posterior uncertainty reduction in the control space.

\subsubsection{Uncertainty assimilation experiments}

Different uncertainty assimilation experiments were designed to explore the effects of different observation types, locations and assimilation times. The different synthetic observation systems are listed in Table 5.1. The rectangular array of surface elevation data represents wide and coarse resolution altimetry of Drake Passage area, which in our barotropic framework is equivalent to array of bottom pressure recorders, roughly resembling the LDA observation coverage in cDrake experiment (Meredith et al. 2011). The linear transect of the Passage represents the typical hydrographic sections, such as WOCE SR1b. Without the current data the Drake transect represents a line of bottom pressure recorders such as used in lower spatial resolution in WOCE experiments (Meredith et al. 2011). The transect is shifted westward to the open ocean along the same latitude to compare the effects of the geometry of the Passage with the dynamics unconstrained by coastlines. The transect is also shifted northward to test the effects of assimilating very remote data in a different oceanographic regime.

The observation-model misfit cost functions are weighted with observation uncertainties (2.2.1). The assumed uncertainty of observations data is equal for all grid points and times, given by the standard errors $\Delta \eta=0.01 \mathrm{~m}$ for altimetry and $\Delta u=0.001 \mathrm{~m} / \mathrm{s}, \Delta v=0.001$ $\mathrm{m} / \mathrm{s}$ for barotropic flow vector components. No cross-correlation of observation uncertainty is assumed and a diagonal observation covariance matrix (2.2.4) is constructed with squares of the assumed standard errors. The size of the observation 
covariance matrix is given by the square of the number of scalars in each observations vector, as listed in Table 5.1, which is much smaller than $86400^{2}=7.5 \cdot 10^{9}$ elements of the Hessian matrix. This Hessian would require 56 GB for storage in double precision and for more realistic GCM configurations is expected to require orders of magnitudes more. In our numerical implementation the full Hessian is not stored, but efficiently reconstructed based on the reduced rank Hessian compression algorithm (2.7.7).

Table 5.1 Table of different synthetic observation system configurations - location and data types.

\begin{tabular}{|l|c|c|c|}
\hline Observation system & $\begin{array}{c}\text { Longitude } \\
\text { coordinate range }\end{array}$ & $\begin{array}{c}\text { Latitude } \\
\text { coordinate range }\end{array}$ & $\begin{array}{c}\text { Number of } \\
\text { scalars }\end{array}$ \\
\hline Drake Passage array altimetry & W 75 - W 59 & S 67 - S 55 & 63 \\
\hline Drake transect altimetry & W 67 & S 65 - S 57 & 5 \\
\hline South Pacific transect altimetry & W 155 & S 65 - S 57 & 5 \\
\hline North Pacific transect altimetry & W 155 & N 17 - N 25 & 5 \\
\hline Drake transect altimetry and flow rate & W 67 & S 65 - S 57 & 15 \\
\hline
\end{tabular}

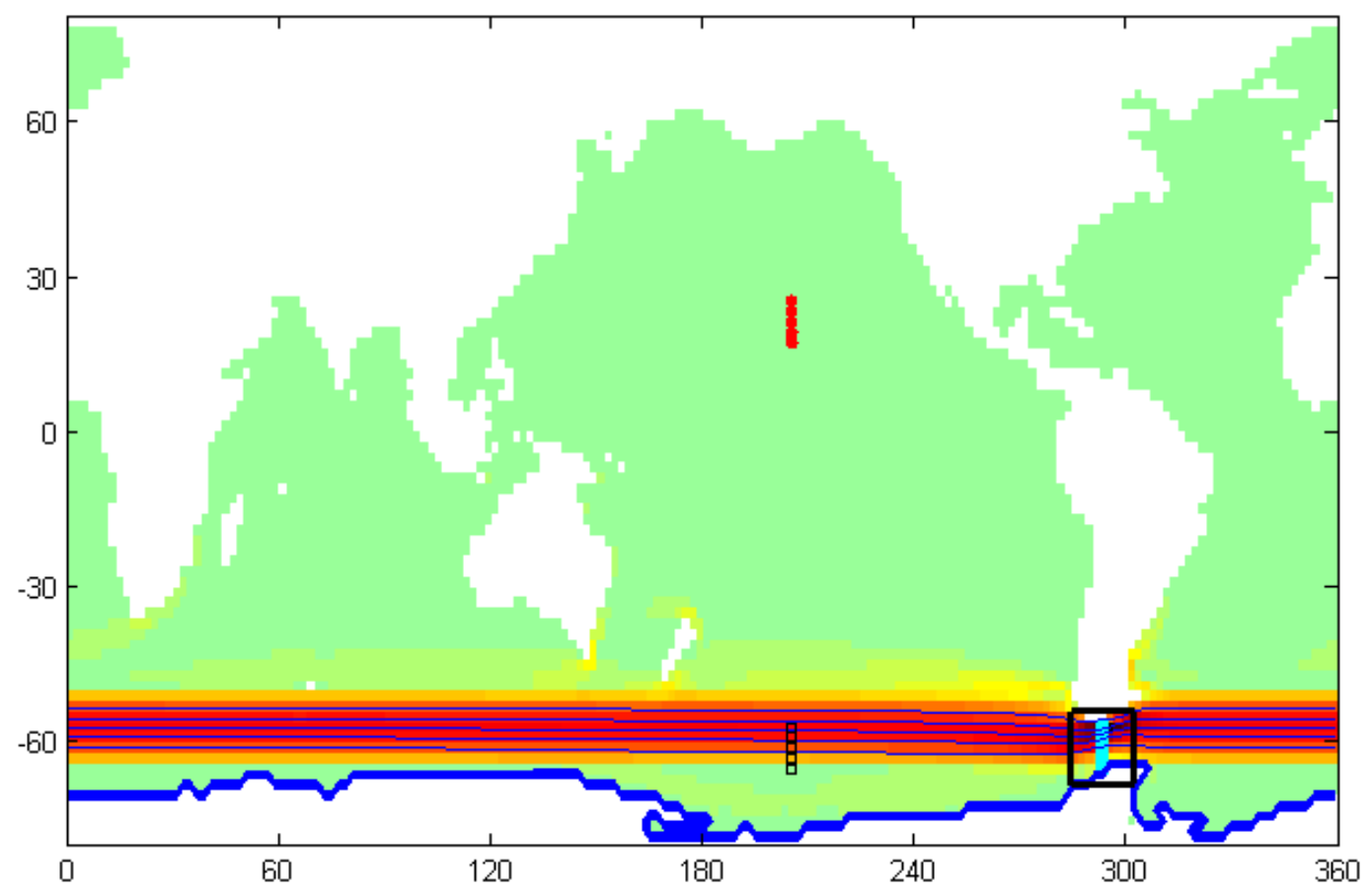

Figure 5.18 Location of observation systems drawn over the steady state flow and sea surface height plot for the flat bottom configuration. The Drake Passage array of 9x7 altimetry data point is shown with back rectangle. The meridional transects are shown with cyan, black and red markers for the Drake, south Pacific and north Pacific transects. 


\subsubsection{Hessian calculation and diagonalization}

The first steps in our inverse uncertainty propagation methodology are calculation of the Hessian matrix and its eigenvalue decomposition (2.7.1) with Lanczos algorithm (Lehoucq et al. 1998). The resulting eigenvector fields resolve the uncertainty structures of model controls in algebraically orthogonal combinations of the control variables (see a simple illustration in Figure 2.2). The eigenvectors corresponding to the larger Hessian eigenvalues represent control fields combinations well constrained by the observations, with the inverse of these eigenvalues equal their estimated uncertainty variance. The inverse of square roots of the eigenvalues are the corresponding standard errors. The eigenvectors with zero or negligibly small eigenvalues, corresponding to infinite uncertainty, are in the nullspace of the uncertainty assimilation Hessian and represent the unconstrained combinations of controls. Note, that this uncertainty assimilation analysis is independent of prior uncertainty assumptions and therefore resolves the pure uncertainty dynamics as captured by the Hessian model. Prior uncertainty assumptions, on the other hand, may introduce bias into the estimation problem and which may be arbitrary if the priors are arbitrarily selected. The prior uncertainty assumptions also lead to posterior uncertainty estimates that are different from the pure assimilated uncertainty (as illustrated in Figure 2.1). These posterior uncertainties depend on the priors and therefore reflect the arbitrariness in prior selection. The following uncertainty reduction sub-section (Section 5.2.3) illustrates in detail the complex dependence of the resulting posterior uncertainties on arbitrary prior choice. However, in this section we eliminate this undesirable effect and focus on the pure assimilated uncertainty dynamics.

\subsubsection{Realistic geometry with flat bottom}

Different inverse uncertainty propagation experiments were compared for each of the observation systems listed in Table 5.1. For each uncertainty assimilation configuration the observation data was assimilated at different time shifts from the time of the initial conditions. It was found that inverse uncertainty propagation depends on the uncertainty assimilation time similarly to sensitivity fields dependence on the adjoint time (4.2.5). Uncertainty is not static and evolves dynamically through transient states even when the forward model is integrated from the steady state. The uncertainty was found to reach a 
steady state on the time scale of the forward model relaxation, consistent with our analytical analysis in Section 4.2.

The spectra of the Hessians for uncertainty assimilated 1 day, 10 days and 90 days after steady state initial conditions are compared in Figure 5.19 for the flat bottom experiment. The spectrum steepens as function of uncertainty assimilation time. The 90 days' leading eigenvalues are larger and the trailing eigenvalues are smaller than the shorter assimilation period ones. This means that the steady state assimilated information gain is more strongly associated with the more constrained eigenmodes of uncertainty.

All the spectra are characterized by a sharp spectral drop after the first 50 eigenvalues. This spectral cut-off separates the range from the nullspace of the Hessians, allowing to restrict the UQ analysis to the dimensionality of the range. Only relatively few Hessian eigenvectors are required for inverse uncertainty propagation, eliminating the necessity to calculate most of the eigenvectors. The effective rank of the Hessian matrix is much smaller than the dimension of the space spanned by its columns. This allows a reduced rank representation of the Hessian and is the foundation of the compressed algorithm of Hessian matrix inversion (2.7.7). Physically, the compactness of the Hessian spectrum implies that the number of dynamically independent observations projecting a nonzero information on model controls is small. Only these meaningful observations constrain the uncertainty of controls, and only they are required for the inverse uncertainty propagation calculation.

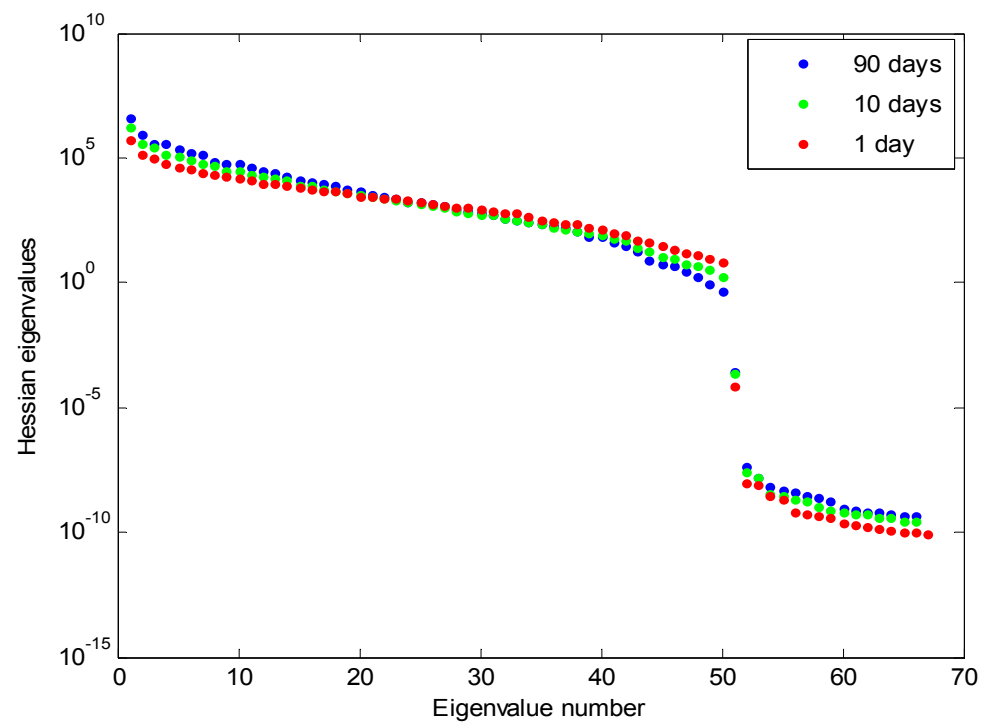

Figure 5.19 Hessian spectra of Drake Passage array altimetry misfits for $\mathrm{f}$ lat bottom configuration, shown for observations assimilated after 1 day. 10 days and 90 days. 
The leading constrained eigenvectors for each of the three uncertainty assimilation times, 1 day, 10 days and 90 days, are shown in the following figures using the control fields panels arrangement as in Figure 5.2. For short uncertainty assimilation time (Figure 5.20) the fields exhibit large planetary scale structures which appear to be proportional to the corresponding sensitivity fields (Figure 5.11), supporting our analytical analysis (4.1.13). However, the detailed local structure at the Drake Passage is very different, e.g. the strong meridional dipole of the uncertainty of zonal wind stress vs. its homogeneous positive sensitivity area. The difference is explained because the sensitivity is the derivative of the integral transport through the Passage, while the uncertainty is due to the sum of misfits of local surface elevation observations. Both, being dynamically linked at the Passage, radiate similar patterns to the far field. But locally the eigenvector decomposition extracts the orthogonal contributions to the Jacobian of the misfits, which are different from the Jacobian of the transport integral.

The orthogonal fields of the Hessian eigenvectors are the principal uncertainty patterns. Assuming an equivalence of backward uncertainty propagation time $t_{0^{-}} t$ with forward time of uncertainty assimilation $t-t_{0}$, since the forward model is integrated from the steady state, allows to describe the evolution of the constrained uncertainty fields. Just as the sensitivities to forcing and initial conditions had opposite dynamic - the initial conditions sensitivities decaying to zero and the sensitivities to wind stress and bottom friction growing to steady state values, similar dynamics can be noticed also for the uncertainties. The relative magnitude of the eigenvector fields shifts from the initial conditions uncertainty to the stress and friction fields, but the effect is less pronounced. Also wavy structures can be noticed after 10 days of backward uncertainty propagation (Figure 5.21), whose spatial scale decreases as the uncertainties evolve backwards (Figure 5.22). These waves are related to the adjoint waves examined in the sensitivity analysis, but their appearance in the Hessian eigenvector fields is a manifestation of "uncertainty waves" propagation through the domain. The south to north dipole asymmetry of the uncertainty patterns across the Drake passage is noticeable for all times for most of the Hessian eigenvector fields, with particularly strong signature of bottom friction uncertainty on the northern side. Other noticeable geographical patterns include the 
circumpolar band of the bottom friction uncertainty and the transient teleconnections patterns of the initial conditions fields.
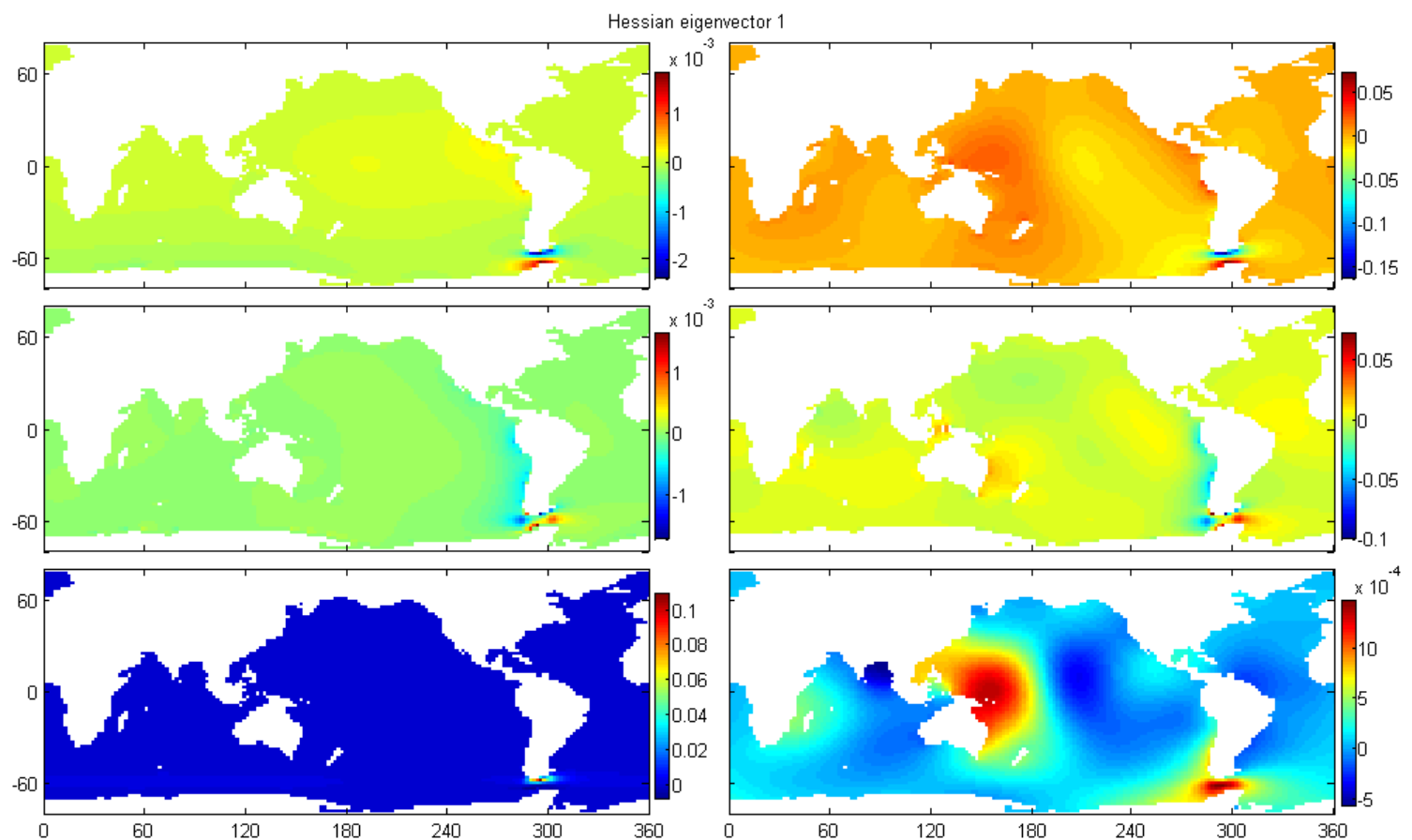

Figure 5.20 Leading eigenvector of the Hessian of Drake array altimetry observations misfit for flat bottom configuration for uncertainty assimilation after 1 day.
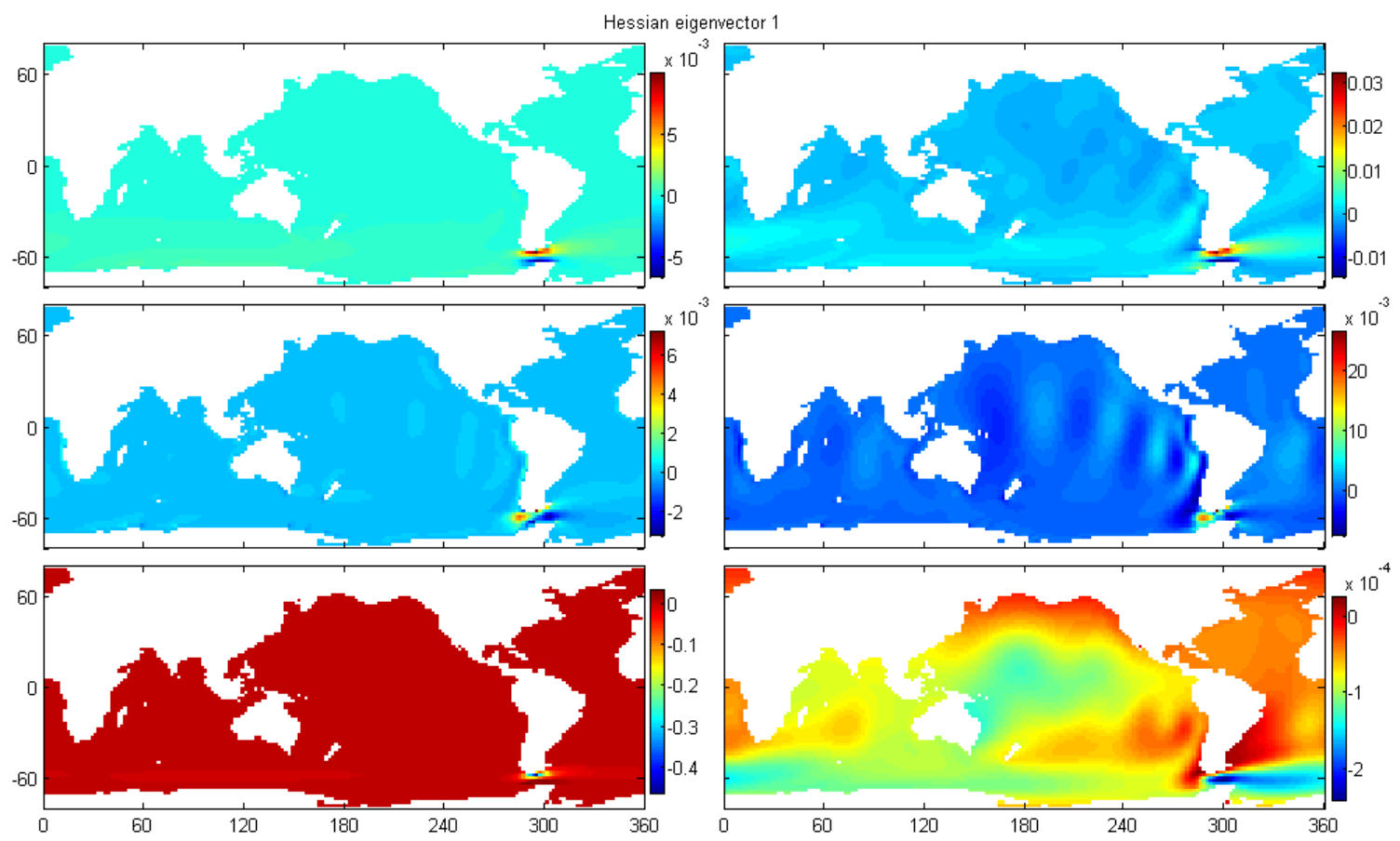

Figure 5.21 Leading eigenvector of the Hessian of Drake array altimetry observations misfit for flat bottom configuration for uncertainty assimilation after 10 days. 


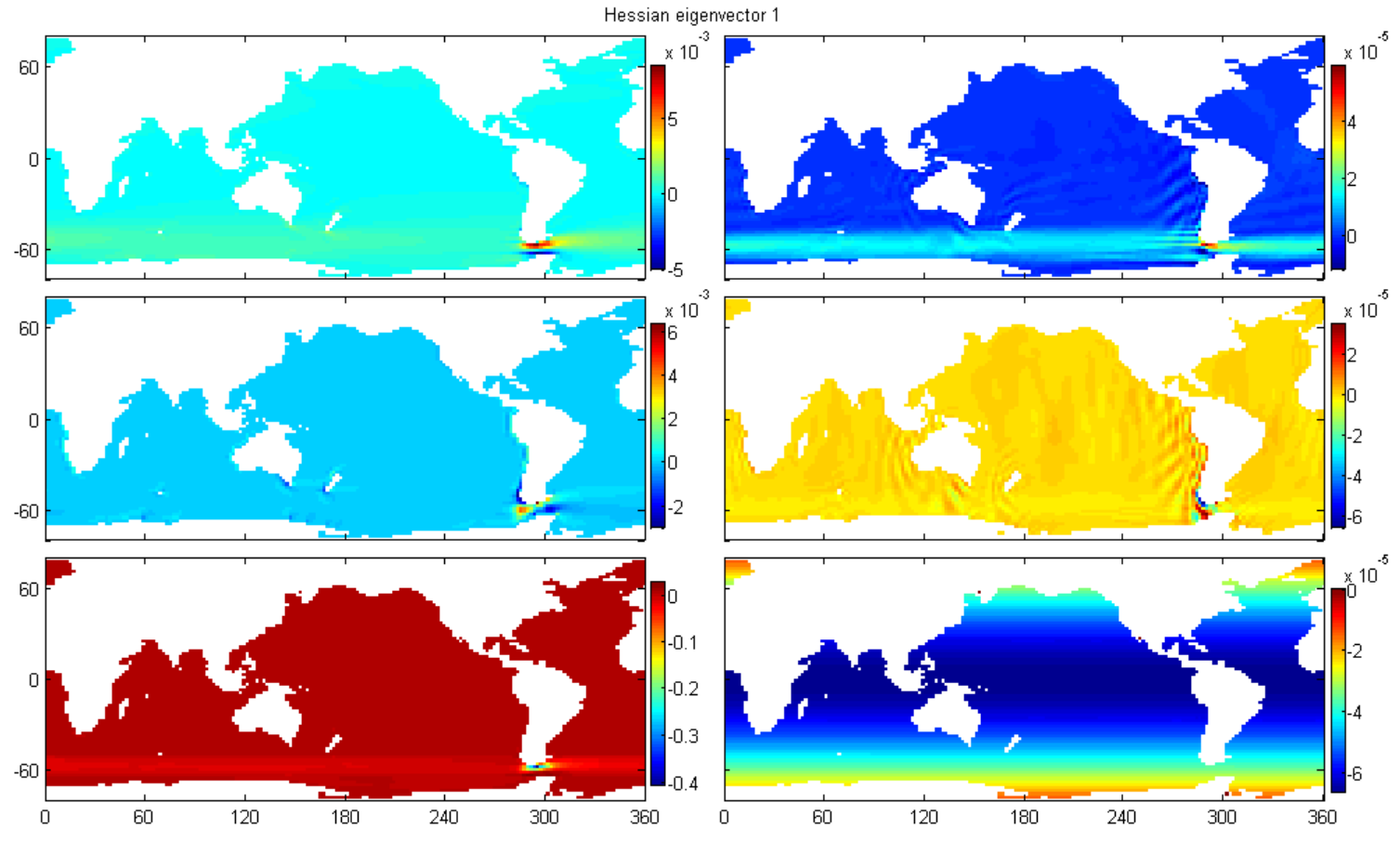

Figure 5.22 Leading eigenvector of the Hessian of Drake array altimetry observations misfit for flat bottom configuration for uncertainty assimilation after 90 days. 


\subsubsection{Realistic bathymetry}

Comparing inverse uncertainty propagation calculations for the realistic bathymetry configuration in the following figures shows qualitatively similar results to the flat bottom case. The increasing tilt of the Hessian spectra with assimilation time is still apparent, although the leading eigenvalue of the 1 day experiment is larger. The patterns of the leading eigenvectors again resemble the sensitivities in the far field, but significantly differ locally. The shift of the signal magnitude from the initial conditions to the forcing is more pronounced. A noticeable difference is the alignment of the uncertainty patterns with the bottom topography, which means that the uncertainty is dynamically steered by the geostrophic contours due to conservation of potential vorticity. In the steady state the strongest signatures of forcing and friction uncertainty are localized at the Drake Passage and downstream. The initial conditions uncertainties exhibit wavelike transient features along the equator with shorter meridional extent than in the flat bottom case. These can be explained by equatorial Rossby uncertainty waves (see Section 5.2.2.1) dynamics over varying and shallower ocean depth. These barotropic waves are constrained in north-south direction by the shorter Rossby radius of deformation, which scales the width of the equatorial wave guide. Other strong signals are noticeable along the Antarctic coast propagating westward in the direction opposite to the adjoint Kelvin waves and on time scales too long for gravity oscillations. The mechanism of these signals is not understood but may be potentially attributed to the numerics of the C-D scheme used to stabilize the realistic bathymetry simulations. 


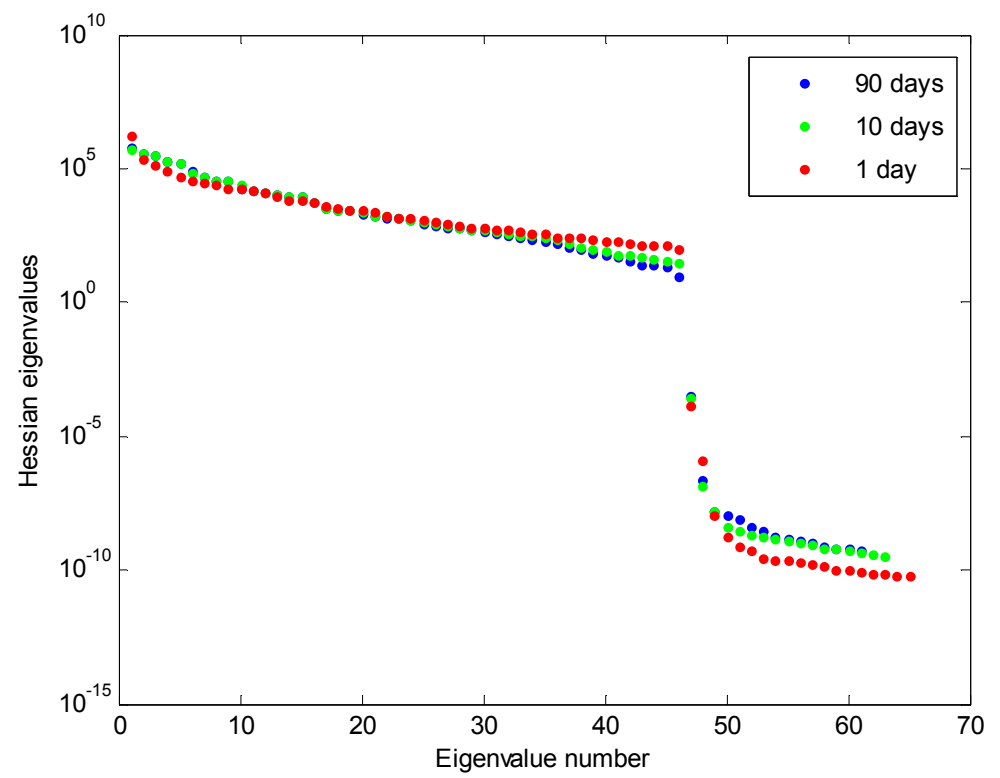

Figure 5.23 Hessian spectra of Drake Passage array altimetry misfits for realistic bathymetry configuration, shown for observations assimilated after 1 day. 10 days and 90 days.

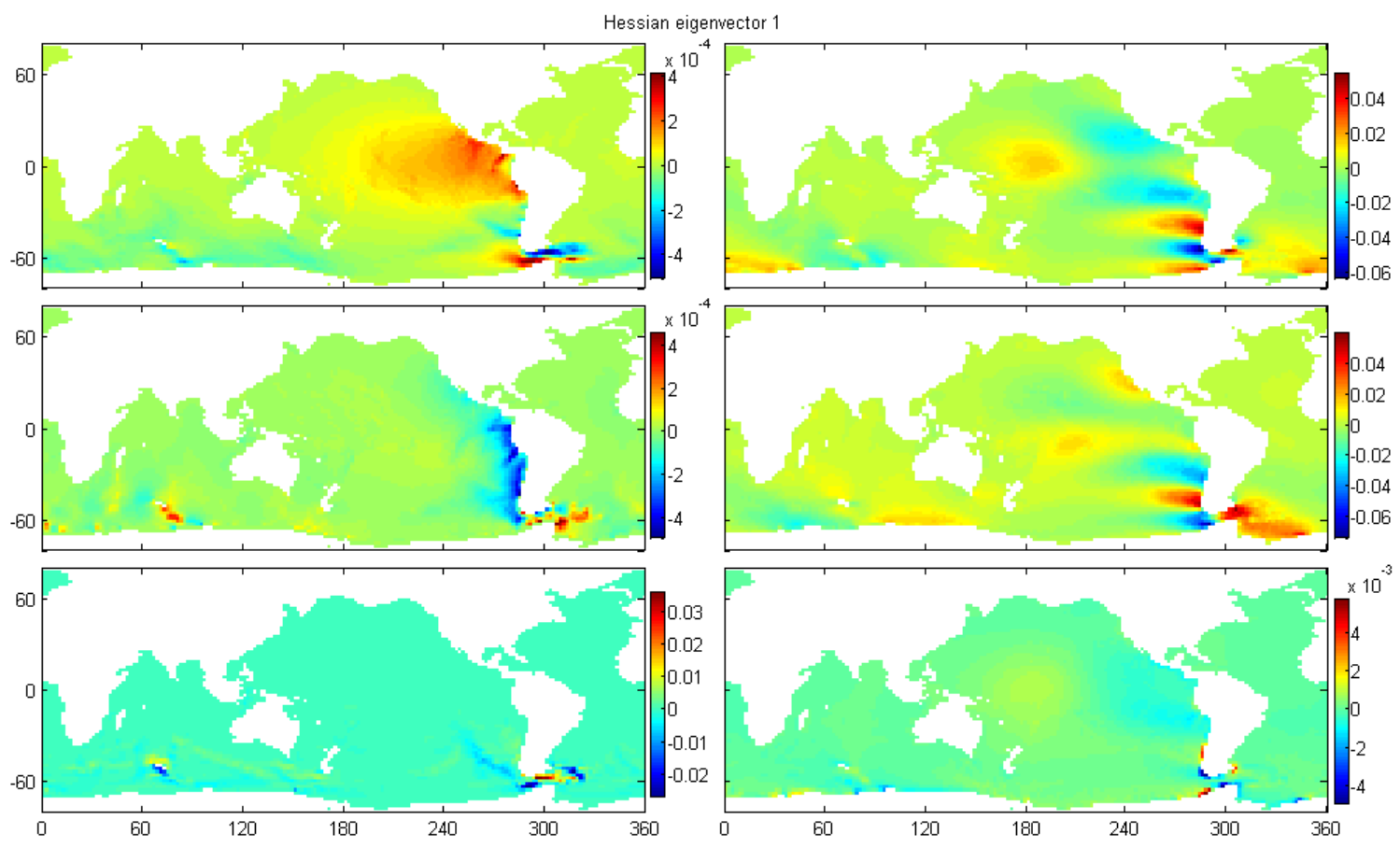

Figure 5.24 Leading eigenvector of the Hessian of Drake array altimetry observations misfit for realistic bathymetry configuration for uncertainty assimilation after 1 day 

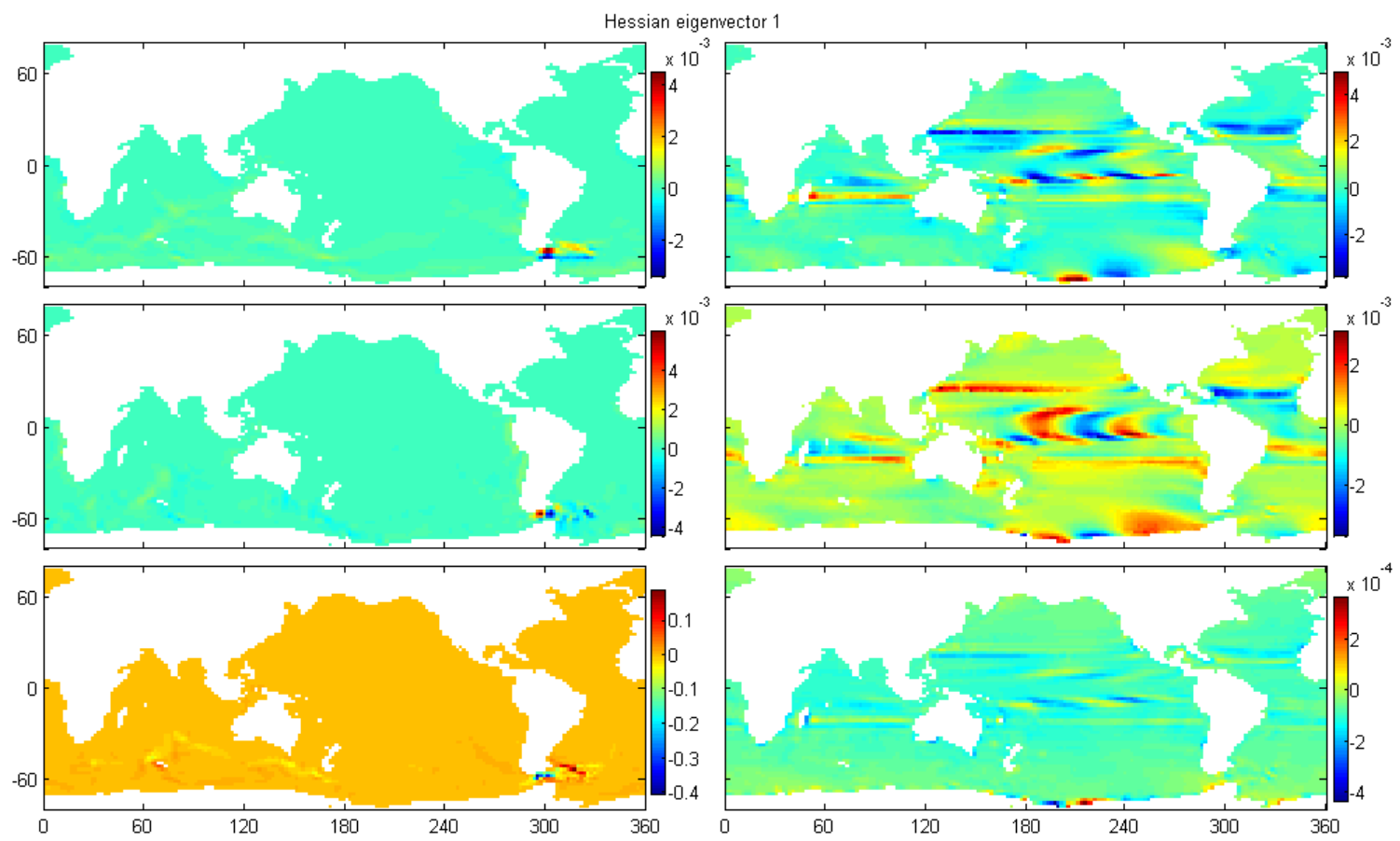

Figure 5.25 Leading eigenvector of the Hessian of Drake array altimetry observations misfit for realistic bathymetry configuration for uncertainty assimilation after 10 days

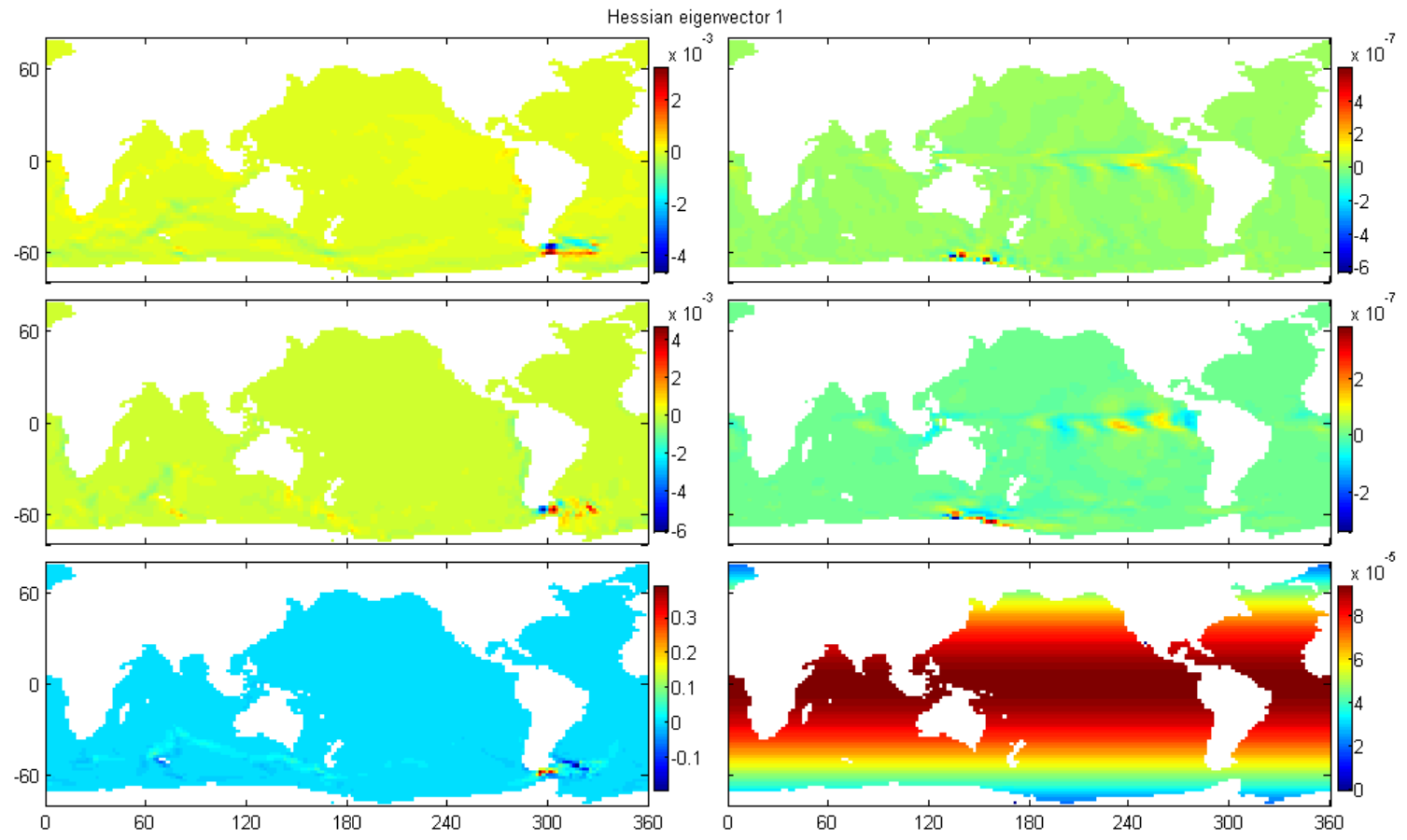

Figure 5.26 Leading eigenvector of the Hessian of Drake array altimetry observations misfit for realistic bathymetry configuration for uncertainty assimilation after 90 days 


\subsubsection{Prior vs. posterior uncertainty: uncertainty reduction}

This section summaries the application of the developed methodology for uncertainty reduction based on model-data misfit Hessian information. Assumed prior uncertainties of model controls are reduced by combination with assimilated uncertainty of synthetic observations. The resulting large regularized information matrix is inverted to obtain the posterior uncertainty covariance matrix according to (2.7.5), implemented for application in very large uncertainty quantification problems. Based on the cut-off of the Hessian spectrum (Figure 5.19) only few Hessian eigenvectors are explicitly resolved with the Lanczos algorithm and are combined with the prior uncertainty matrix in the developed matrix-free algorithm based on (2.7.8).

The following figures display the reduction in the uncertainty of control fields for different uncertainty assimilation times, different synthetic observation systems (Table 5.1) and two forward model configurations. A key feature of these calculations is dependence on the assumed prior uncertainties. If these are not a priori known or estimated from trusted statistics, the uncertainty reduction results retain the arbitrariness of the prior selection, which is demonstrated below. In the following examples the prior uncertainties are not correlated, their covariance matrix is diagonal with constant values for each of the six different physical fields of the control vector. The prior uncertainty diagonal is given by the squares of the corresponding standard errors

$$
\left[\Delta \tau^{x}, \Delta \tau^{y}, \Delta r, \Delta u_{t=0}, \Delta v_{t=0}, \Delta \eta_{t=0}\right]^{T},
$$

that is a single constant scalar value for each of the panels on Figure 5.2. The posterior uncertainty matrix is not diagonal, but because it is very large (7.5.10 elements) only its diagonal is analyzed here, representing the posterior uncertainty variances of each of the elements of the control vector. In the following figures we compare the prior and posterior diagonals in terms of the posterior relative error reduction as defined by (2.7.11) or equivalently by defining relative uncertainty reduction percentage

$$
\mathrm{RURP}=\frac{\Delta_{\text {prior }}-\Delta_{\text {post }}}{\Delta_{\text {prior }}} \cdot 100
$$




\subsubsection{Realistic geometry with flat bottom}

Consider first the flat bottom configuration with Drake Passage array altimetry observations. The relative uncertainty reduction for uncertainty assimilation time 1 day for prior uncertainties given by $\left[\Delta \tau^{\mathrm{x}}, \Delta \tau^{\mathrm{y}}, \Delta r, \Delta u_{t=0}, \Delta v_{t=0}, \Delta \eta_{t=0}\right]^{T}=[0.1 \mathrm{~Pa}, 0.1 \mathrm{~Pa}$, $5.0 \mathrm{e}-3 \mathrm{~m} / \mathrm{s}, 0.01 \mathrm{~m} / \mathrm{s}, 0.01 \mathrm{~m} / \mathrm{s}, 0.1 \mathrm{~m}]^{T}$ are shown in Figure 5.27 . The uncertainty reduction is localized in the Drake passage area, only slightly extending beyond the geographic coverage of the assimilated altimetry data. An exception is the reduction of the initial surface elevation uncertainty, which extends far beyond the Drake Passage area and has a strong signature in the western Pacific. This planetary scale uncertainty teleconnection mechanism is explained by the adjoint Kelvin waves (Figure 5.3 and Figure 5.11), which radiate from the western Pacific and propagate to the Drake Passage area after 24 hours. Uncertainty of wind stress is reduced by 0.2 to 0.3 percent, the reduction of the initial surface elevation uncertainty is an order of magnitude less. Larger uncertainty reduction of 6 to $8 \%$ is calculated for the initial flow components. Maximum $0.8 \%$ reduction is found for the bottom friction coefficient at the highest reduction point near the northern coast of the Drake Passage. The zonal band of the flat bottom ACC confines the reduction of friction uncertainty, shown by the lowest uncertainty reduction contour (blue). For the other fields some minimal uncertainty reduction extends all over the global ocean area, with the first contour aligned along the continental boundaries.

Comparing the aforementioned results to uncertainty reduction after 10 days (Figure 5.28) shows larger reduction for the forcing and friction fields and smaller for the initial conditions. The reduction of uncertainty of wind stress is 4 to $8 \%$, of bottom friction up to $12 \%$, for initial velocities less than $1 \%$, and order of $10^{-3}$ for the initial surface elevation. This uncertainty dynamics is consistent with evolution of sensitivity and Hessian eigenvector fields. As the system evolves away from the initial conditions their effects are "forgotten", while the effects of the boundary conditions grow stronger. This trend continues for 90 days uncertainty assimilation time (Figure 5.29). The reduction of wind stress uncertainty grows for both vector components, but more for the meridional winds, reaching $12 \%$. For bottom friction the reduction is still larger - up to $14 \%$. For the initial conditions the uncertainty reduction is small, and while some localized and physically meaningful patterns can still be observed, the machinery seems to approach 
the limits of finite precision numerics. In practice, the uncertainty reduction of the initial conditions can be neglected for long uncertainty assimilation times, as the uncertainty is expected to evolve to a steady state independent of any initial conditions.

Neglecting the random uncertainty reduction patterns in Figure 5.29 because they are too small $O\left(10^{-8}\right)$ and are attributed to artifacts of finite precision numerics raises the question what is the threshold for neglecting the uncertainty reduction results. The mentioned reduction of $0.2 \%$ in Figure 5.27 might be considered effectively zero for practical applications and it might be argued that observations do not meaningfully constrain the uncertainty of these controls. Nonetheless, the patterns appear spatially coherent with meaningful physical interpretation and therefore are more likely to be considered a signal rather than noise. To make a quantitative discrimination of signal from noise and decide what amount of uncertainty reduction is too small to be considered, one may need to introduce the asymptotic machinery of statistical inference. Assigning formal asymptotic confidence regions and other measures of statistical significance of estimation results is an important next step, but is not pursued in this dissertation.

Next we examine the effects of different priors selections on the resulting uncertainty reduction. Figure 5.29 can be compared to Figure 5.30 and Figure 5.31, showing identical calculations with the only difference in the assumed prior uncertainty of wind stress. The values are $0.1 \mathrm{~Pa}, 0.01 \mathrm{~Pa}$ and $0.0001 \mathrm{~Pa}$ correspondingly. Note that the amplitude of the supplied wind stress profile is $\tau_{0}=0.1 \mathrm{~Pa}$, such that the prior uncertainties are $100 \%, 10 \%$ and $0.1 \%$ of the scale of the forcing. The results show that not only the uncertainty reduction of the wind stress decreases with decrease of its prior, but also it leads to larger reduction of the other uncertainties - most evident here for the bottom friction, reaching $30 \%$. This result highlights the dependence of the uncertainty reduction on the choice of the prior, and also demonstrates the cross-coupling of the different control fields.

We also consider the effects of the different observation systems. Compare the 10 days uncertainty assimilation of Drake Passage array altimetry (Figure 5.28) to Drake transect altimetry (Figure 5.32) and to Drake transect altimetry and current data (Figure 5.33). As it can be expected, the decrease of the amount of assimilated data points reduces the 
effects on the uncertainty reduction. All the uncertainty reduction fields are weaker for the Drake transect altimetry case. They are all stronger for the Drake transect altimetry and currents case, indicating the value of assimilation of different physical variables for constraining the posterior uncertainties. The spatial patterns of uncertainty reduction are collocated with the assimilated grid points, spanning the Drake Passage and radiating to the far field only for the initial surface elevation. The lowest contour is consistent between all three cases, with the band of friction uncertainty improvement confined in similar zonal channels and the minimal uncertainty reduction extending over all the global ocean.

Moreover, compare the uncertainty assimilation effects of the Drake transect altimetry (Figure 5.32) to altimetry of the south Pacific (Figure 5.34) and north Pacific (Figure 5.35) transects. Substantial differences are observed. As can be expected, the uncertainty reduction areas shift geographically following the location of the observations. However, the magnitudes of the reduction are not exactly the same, indicating the effects of different uncertainty dynamics in different regions. Shifting the observations zonally along the path of the ACC to an open area in the southern Pacific slightly increases the local uncertainty reduction of wind stress components and initial zonal velocity. The reduction of uncertainties of initial surface elevation and meridional velocity are slightly decreased. The largest difference is in the reduction of bottom friction uncertainty. In the open ocean uncertainty reduction weakens by more than factor of two, which can be attributed to the constriction effect of the narrow Drake Passage. The flow accelerates through the Passage and the role of bottom friction is larger in that region. Another interesting difference is the shape of initial elevation uncertainty patterns. While the Drake Passage observations radiate to the west Pacific via long Rossby wave teleconnection and also shed gradually decreasing uncertainty reduction downstream along the Antarctic coast, the south Pacific observations' influence spreads both to the east and along a north-east oriented band to the north of the observed area. The downstream band of south Pacific observations' uncertainty reduction is almost fully blocked at the entrance to the Drake Passage, where a local maximum is clearly seen. The diagonal north-east oriented band of uncertainty reduction is interesting because it connects the equatorial and mid-latitude regions. 
Moving the altimetry transect northward to the subtropical northern Pacific also exhibits interesting uncertainty patterns. While the uncertainty reduction effects on wind stress and initial currents are localized and of comparable magnitude, albeit weaker for the meridional stress and the velocities, the uncertainty of surface elevation radiates strongly to the south of the equator and to the Indian ocean, as well as the western coast of south America. Dynamically this might be linked to excitation of a wide asymmetric equatorial barotropic wave due to the mixed Kelvin-planetary mode. The most interesting result is the reduction of uncertainty of the bottom friction: although the topography is flat and while the assimilated data is in the north Pacific, the uncertainty is constrained only in the south along the ACC band and peaking at the Drake Passage.

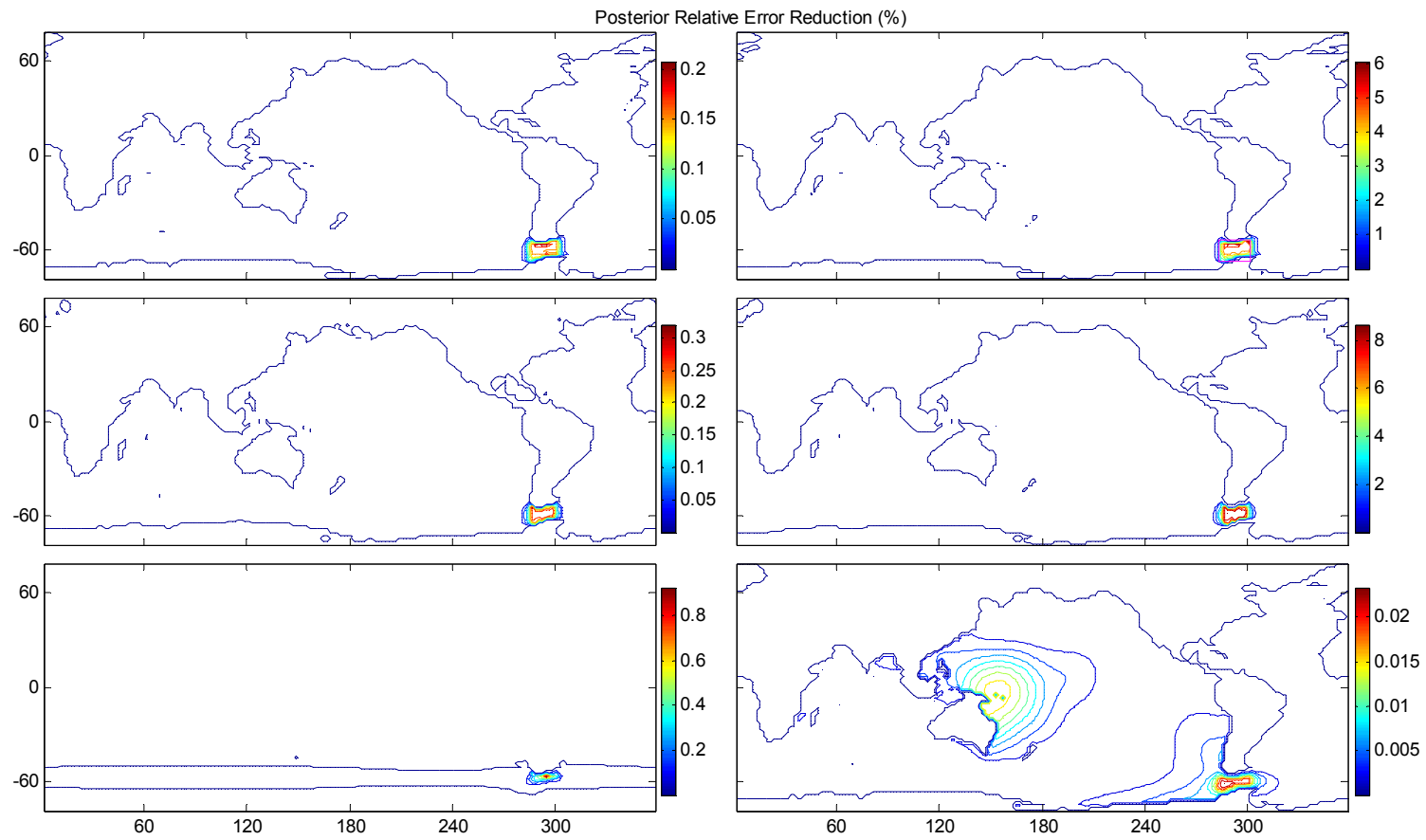

Figure 5.27 Relative uncertainty reduction with Drake array altimetry observations in flat bottom configuration for uncertainty assimilation after 1 day. The prior uncertainties are given by $\left[\Delta \tau^{\mathrm{x}}, \Delta \tau^{\mathrm{y}}, \Delta r\right.$,

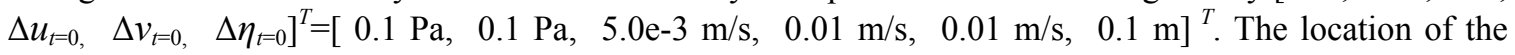
assimilated observations is marked by the magenta rectangle, here shown only on the top right panel. 

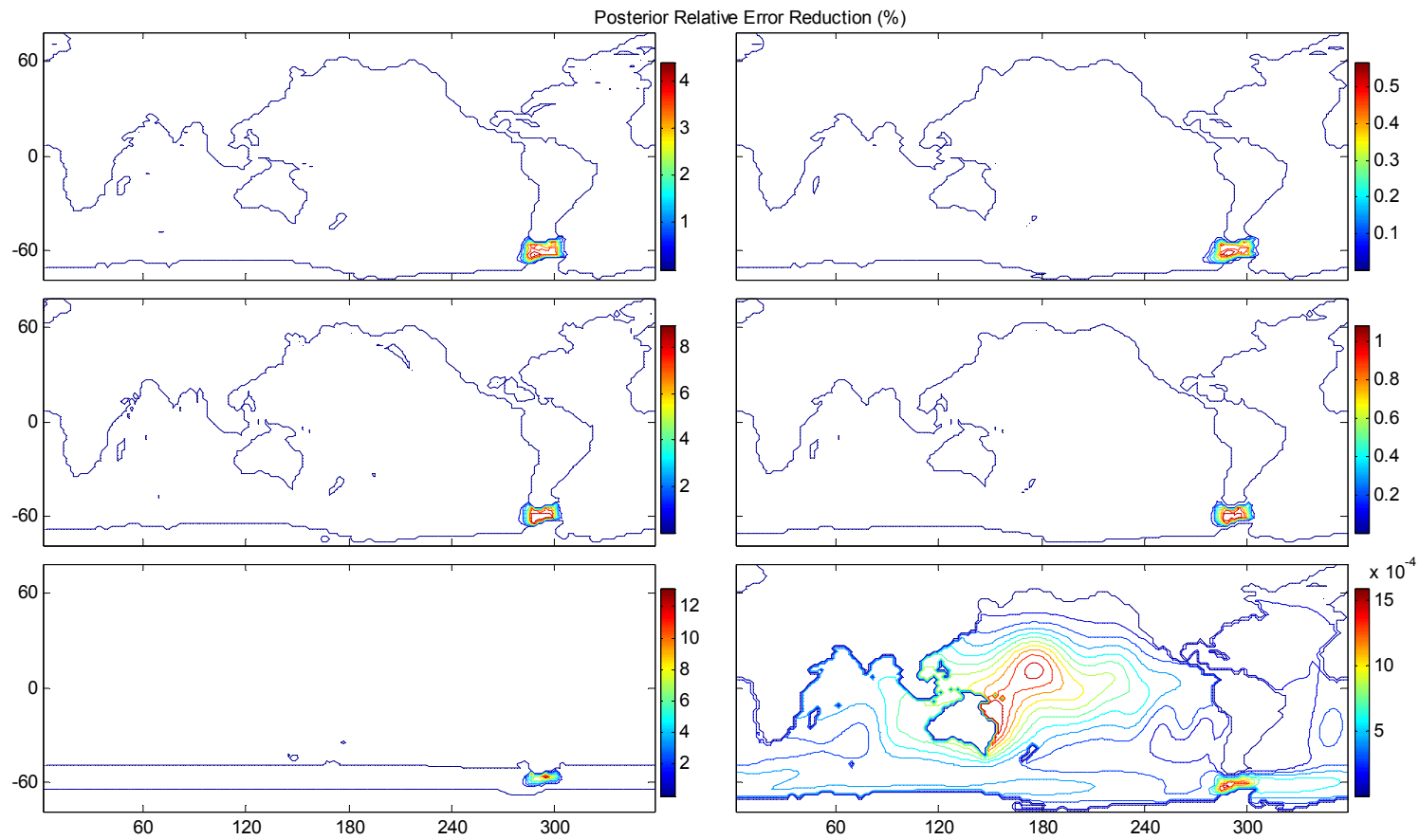

Figure 5.28 Same as Figure 5.27 but for 10 days uncertainty assimilation time.
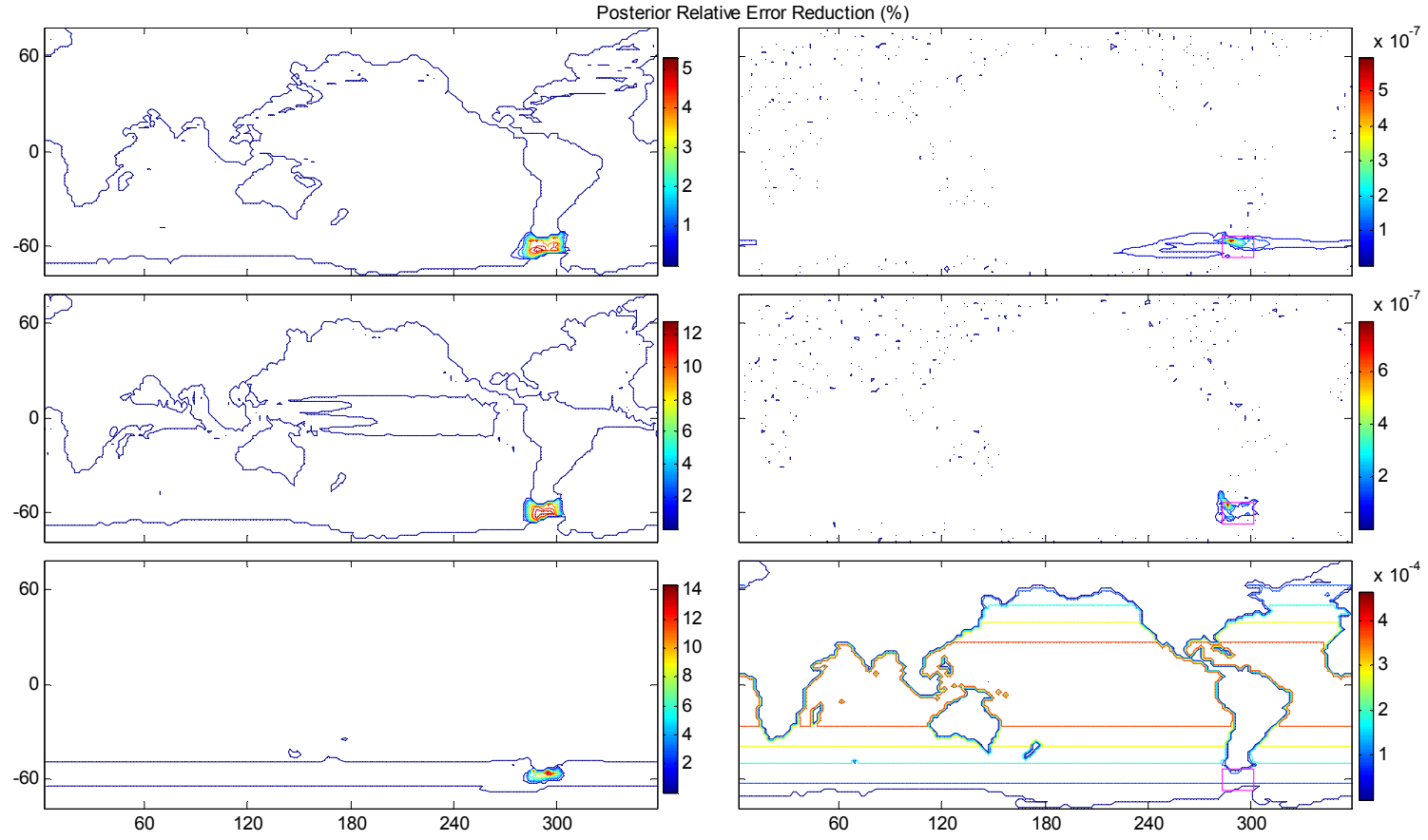

Figure 5.29 Same as Figure 5.27 but for 90 days uncertainty assimilation time. The location of the assimilated observations is marked by the magenta rectangle, here shown on the three right panels. 

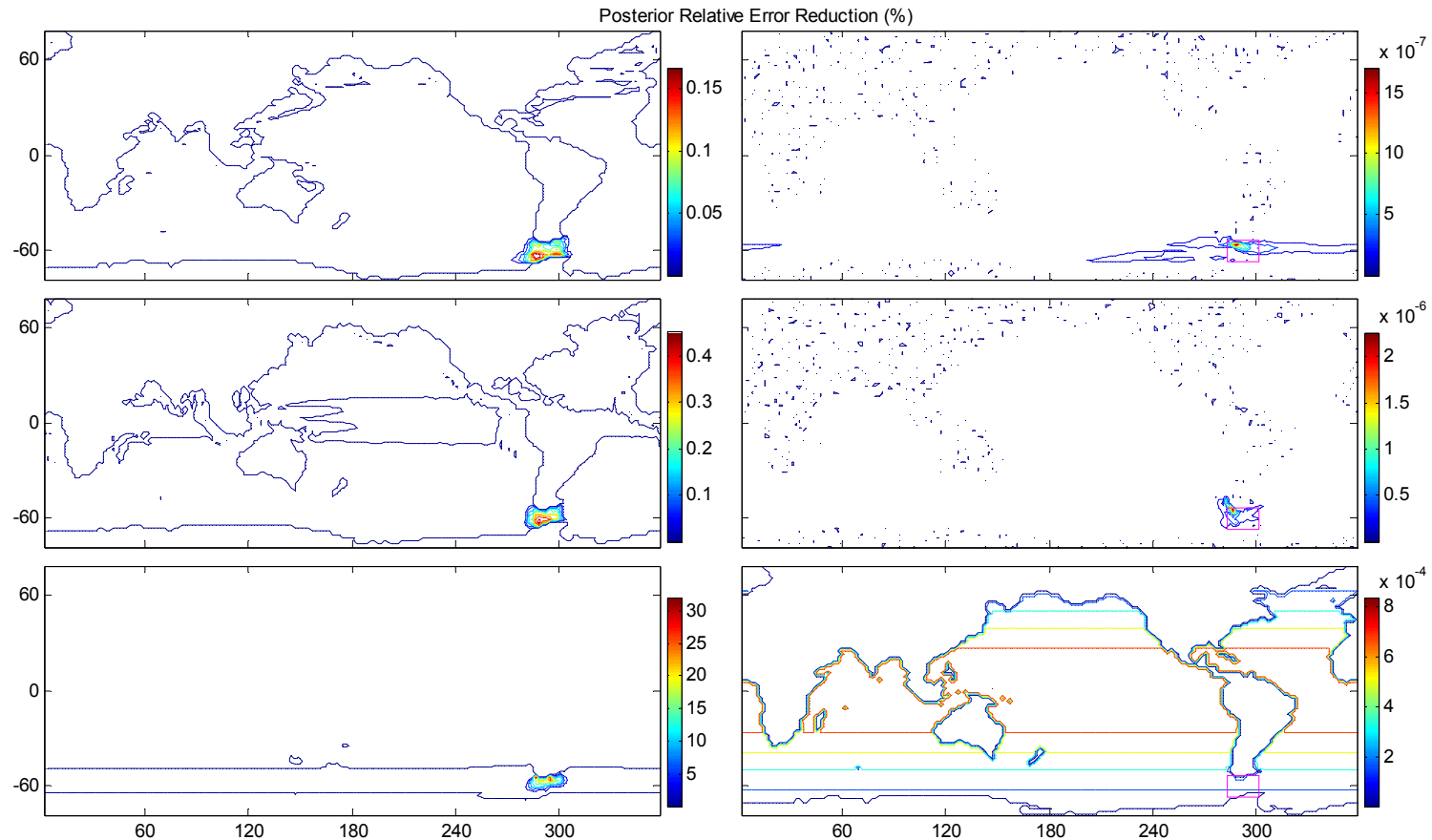

Figure 5.30 Same as Figure 5.29 for 90 days uncertainty assimilation time, but for smaller prior uncertainty

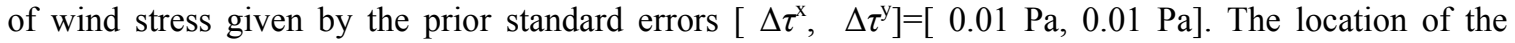
assimilated observations is marked by the magenta rectangle, here shown on the three right panels.

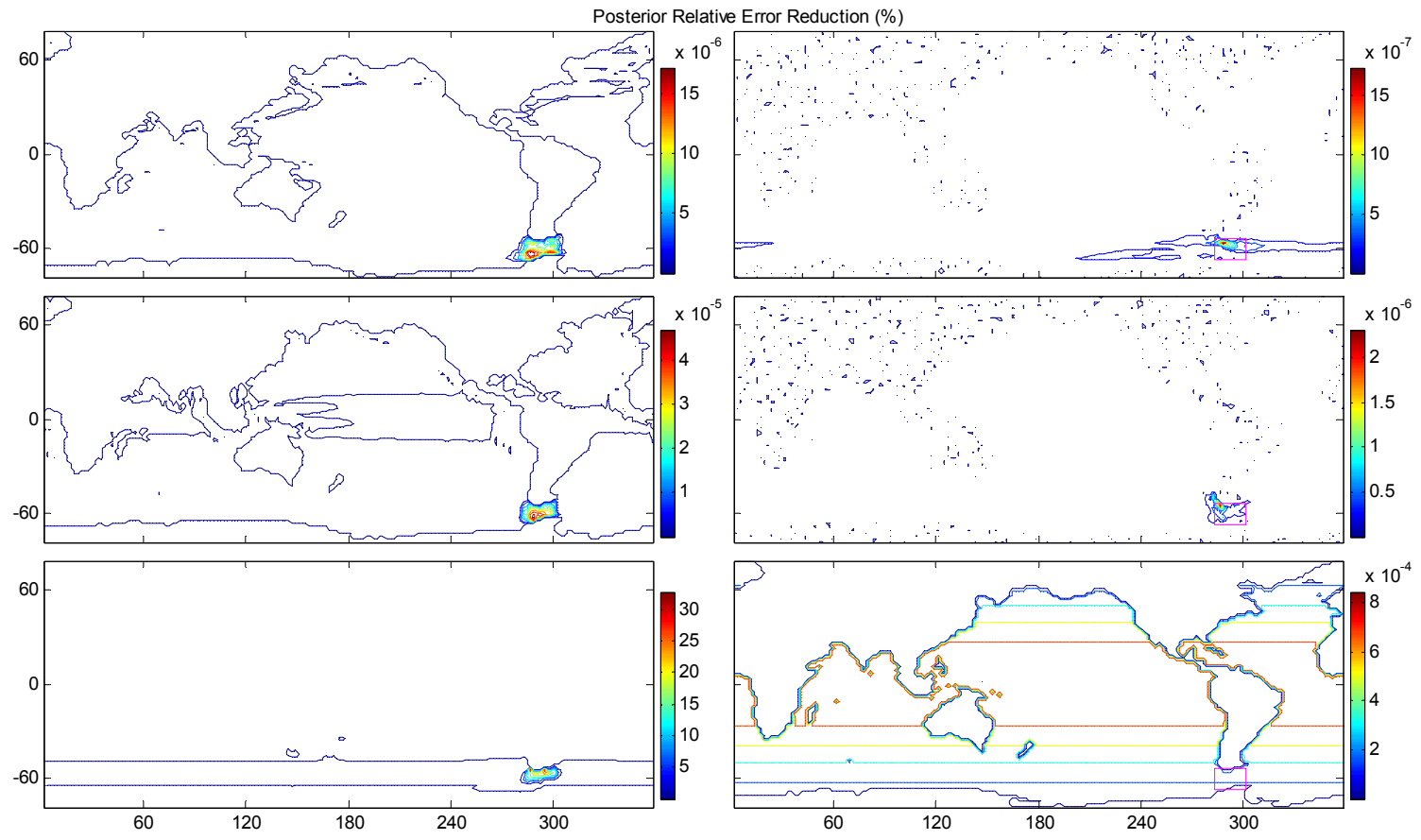

Figure 5.31 Same as Figure 5.29 for 90 days uncertainty assimilation time, but for smaller prior uncertainty of wind stress given by the prior standard errors $\left[\Delta \tau^{\mathrm{x}}, \Delta \tau^{\mathrm{y}}\right]=[0.0001 \mathrm{~Pa}, 0.0001 \mathrm{~Pa}]$. The location of the assimilated observations is marked by the magenta rectangle, here shown on the three right panels. 


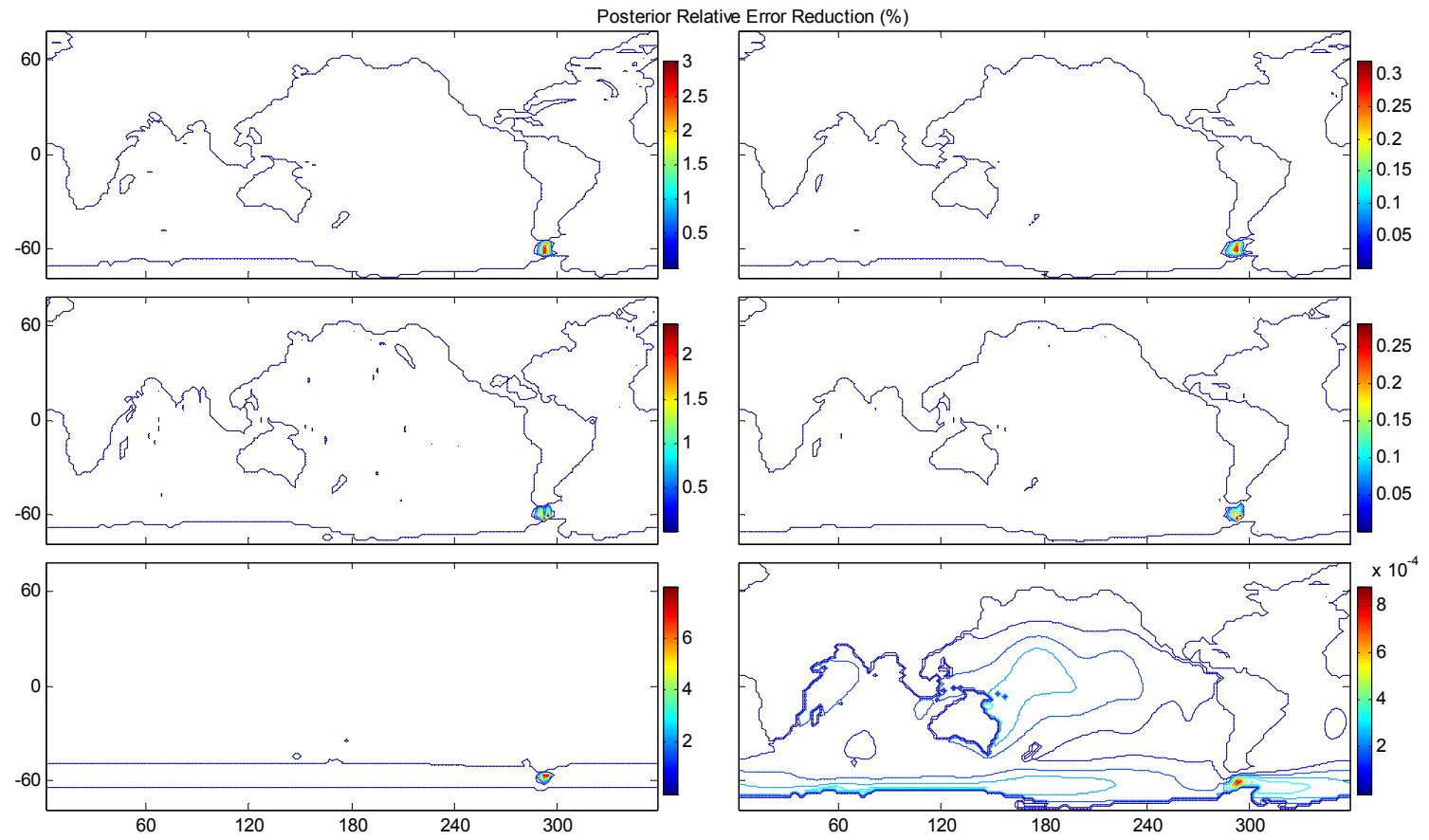

Figure 5.32 Relative uncertainty reduction with Drake transect altimetry observations in flat bottom configuration for uncertainty assimilation after 10 days. The prior uncertainties are same as in Figure 5.27

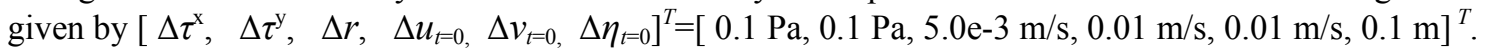
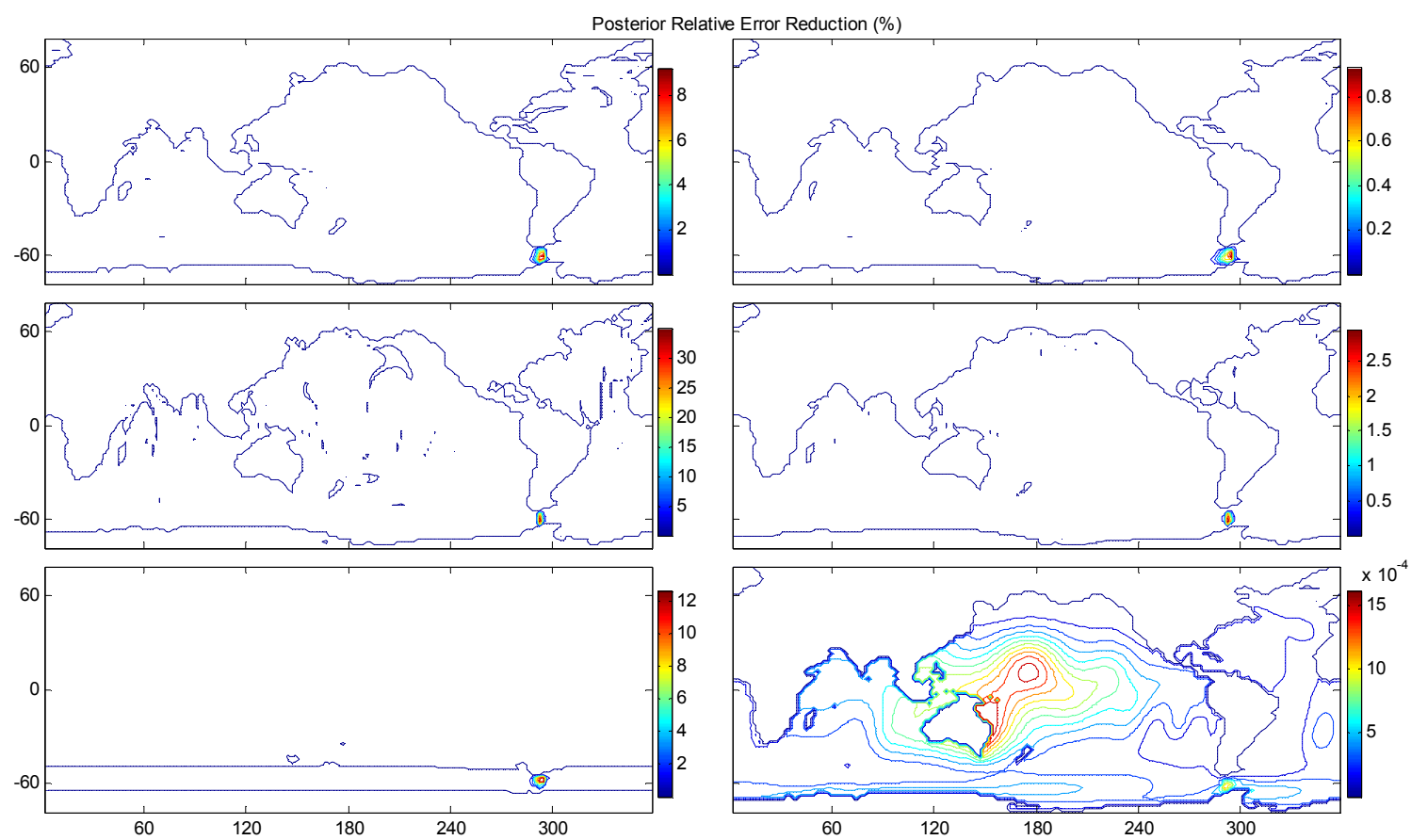

Figure 5.33 Same as in Figure 5.32 for 10 days uncertainty assimilation time, but with Drake Transect Altimetry and Currents data. 


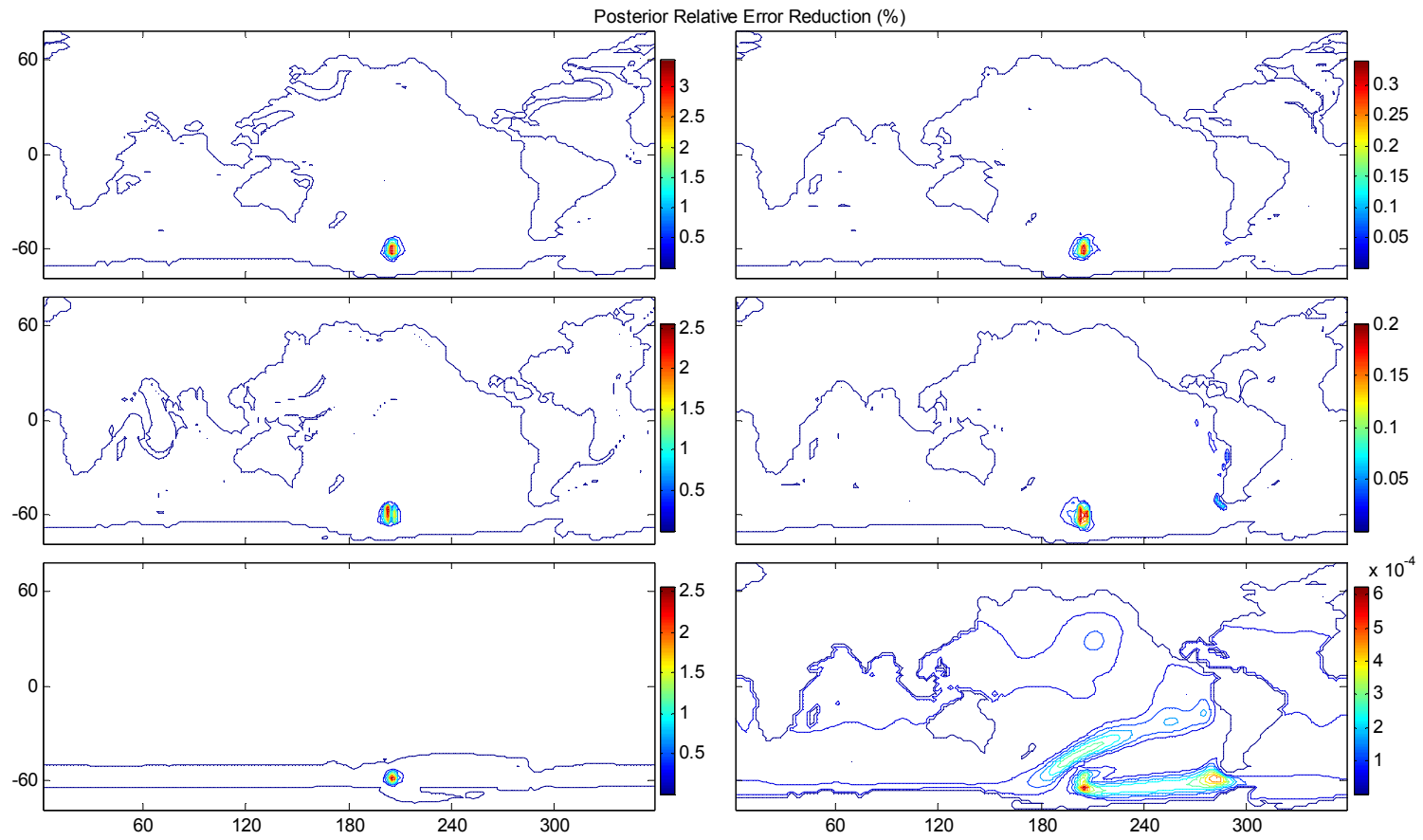

Figure 5.34 Same as in Figure 5.32 for 10 days uncertainty assimilation time, but with south Pacific transect altimetry data.

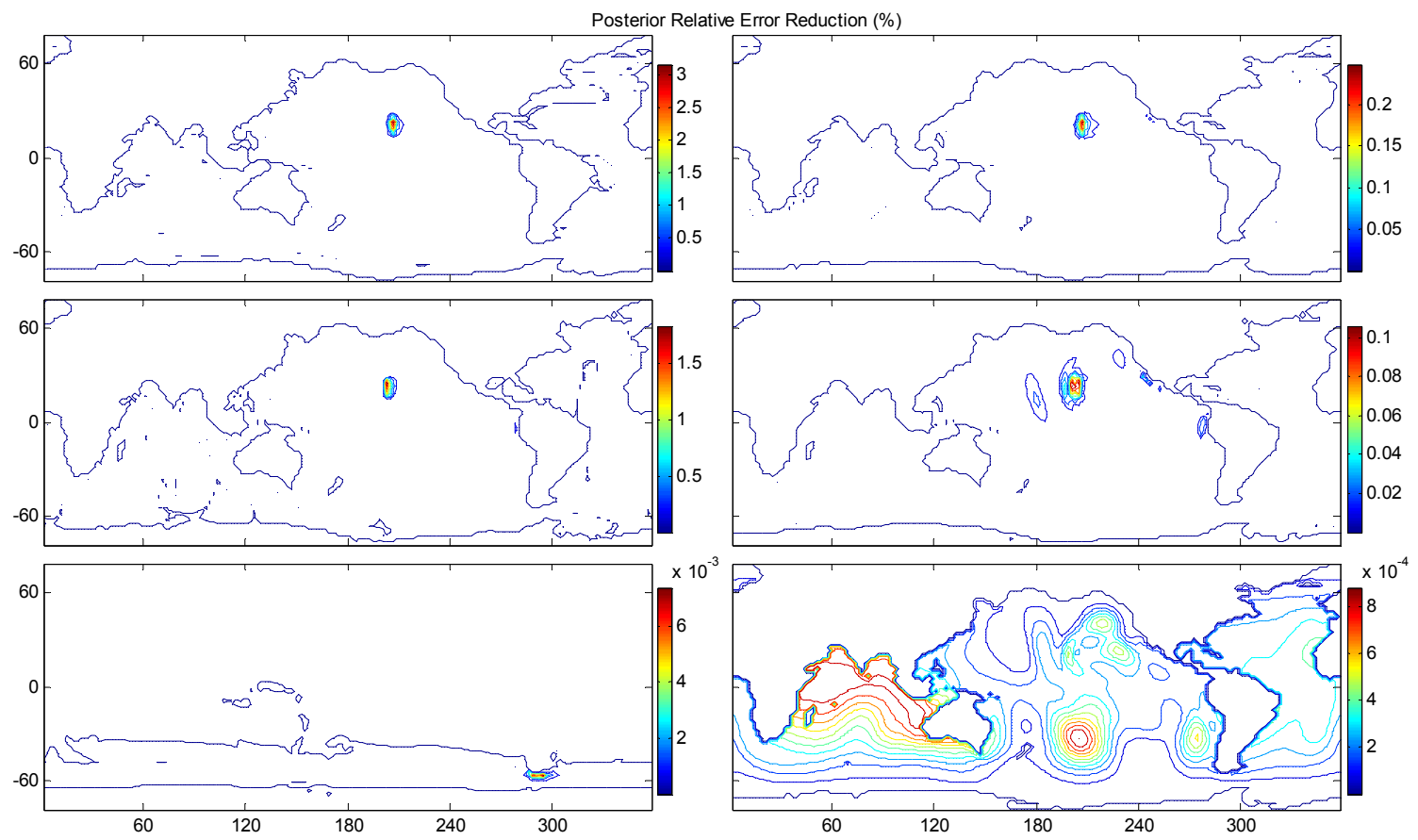

Figure 5.35 Same as in Figure 5.32 for 10 days uncertainty assimilation time, but with north Pacific transect altimetry data.

\subsubsection{Realistic bathymetry}

Next we examine the uncertainty reduction patterns for the realistic bathymetry configuration, as shown in the following figures. The patterns overall resemble to the flat 
bottom case, although much larger in magnitude for forcing and friction and less localized with complex spatial structure for the initial conditions. The contours of uncertainty reduction extend outside the assimilated data region for all control fields for 1 day uncertainty assimilation period (Figure 5.36). The reduction of zonal wind stress uncertainty extends farther downstream than upstream and reaches maximum $11 \%$ at discrete points at northern and southern sides of the Drake Passage. The uncertainty reduction of the meridional wind stress is much stronger, reaching $46 \%$ at a discrete point on the southern side. Uncertainty reduction for the bottom friction reaches $3 \%$, which is much more than the corresponding $0.8 \%$ for the flat bottom configuration. Uncertainty reduction for the initial velocities is of comparable magnitude to the flat bottom case, but weaker $2-4 \%$ vs. $6-8 \%$ in the flat bottom example. It is much stronger for the initial surface elevation, $6 \%$ vs. $0.02 \%$. The most significant difference is the complex spatial structure of the uncertainty reduction patterns, consistent for all three initial condition fields. These patterns reach as far as $1500 \mathrm{~km}$ upstream and downstream of the Passage area, the upstream part is adjacent to the southern coast, the downstream part aligned along the northern coast bending and extending to the eastern coast of South America.

Considering the longer uncertainty assimilation periods, the 10 days (Figure 5.37) and 90 days (Figure 5.38) uncertainty reduction patterns converge to the steady state. Reduction of wind stress and bottom friction uncertainties grows, up to $58 \%$ for the meridional wind stress. The band of bottom friction uncertainty reduction covers a wider meridional extent and as Figure 5.38 captures - it follows the topographic features along with the current itself. The prominent feature is the same overall dynamics of uncertainty as in the flat bottom case - the uncertainty reduction of boundary conditions of momentum sources and sinks grows to the steady state, while the for the initial conditions it decays to zero as the systems "forgets" their effects. No uncertainty reduction signature remains in the area of the assimilated observations for the initial velocity and elevation fields. After 10 days some far field signals remain, and after 90 days the UQ machinery resolves only numerical noise for the velocities, while the effects of surface elevation decay more slowly.

The effects of prior uncertainty selection are illustrated next. Comparing Figure 5.39 to Figure 5.38 we see that reducing the prior for wind stress not only decreases the relative 
uncertainty reduction of the stress components themselves, dropping from $58 \%$ to $11 \%$ for meridional wind stress at the upstream points on the northern coast of the Drake Passage, but it also affects the other fields. Just as in the flat bottom case, the uncertainty reduction is larger for the rest of control fields and largest for the bottom friction, reaching $30 \%$. The effect is stronger for the much smaller prior uncertainty of wind stress (Figure 5.40). The reduction of wind stress uncertainty decreases to $O\left(10^{-6}\right)$ and for bottom friction it increases to $35 \%$. The uncertainty reduction for initial surface elevation increases from $O\left(10^{-5}\right)$ to $O\left(10^{-4}\right)$ to $O\left(10^{-3}\right)$, although no direct physical meaning probably should be assigned to its planetary scale meridional profile because of the smallness of its amplitude. For initial velocities some remote signals emerge from the numerical noise.

Next, we compare the 10 days uncertainty assimilation of Drake Passage array altimetry (Figure 5.37) to Drake transect altimetry (Figure 5.41) for the realistic bathymetry configuration. As in the case of the flat bottom topography, decreasing the number of assimilated altimetry data points data from two dimensional array to only one row of grid points decreases the uncertainty reduction patterns coverage and magnitude. For zonal wind stress it drops from $18 \%$ at the discrete points at northern and southern sides of the Drake Passage to 5\% peak in the interior of the Passage. For the meridional stress the drop is from $58 \%$ to $11 \%$. Uncertinty reduction for the bottom friction drops from $4 \%$ to $0.8 \%$. The remote signals of the initial conditions uncertainty reduction are also weakened for the transect compared to the array. We note that the locations of these signals are consistent - equatorial bands for the velocities and few spots along the Antarctic coast for the surface elevations. When compared to the Drake transect with altimetry and the current data (Figure 5.42) the magnitude of uncertainty reduction is stronger when current uncertainty is also assimilated, but not as strong as in the case of 2D altimetry array. The spatial coverage of uncertainty reduction appears very similar for both transect experiments, except that for altimetry and current assimilation the equatorial uncertainty signals for the initial conditions are masked out by the stronger Antarctic coastal signals.

Finally, we examine the effects of translating the assimilated altimetry transect to the middle of the southern Pacific (Figure 5.43) and the subtropical Pacific north (Figure 
5.44). The magnitudes of uncertainty reduction for the wind stress are reversed between the two components depending on the location of the assimilated data. For the northern Pacific transect the zonal wind stress is more strongly constrained, while for the southern Pacific transect it is the meridional wind stress, which is also the case for the Drake Passage transect (Figure 5.41). The bottom friction uncertainty is constrained by the north Pacific observations only in the southern hemisphere, peaking to the south and to the north of the Australian continent. The effect of south Pacific transect on the bottom friction uncertainty is similar to the Drake transect, except that the local pattern is zonally elongated following the ACC core downstream. Uncertainty reduction patterns for the initial conditions are fragmented for both Pacific transects but the magnitude is small. To summarize, these results indicate that uncertainty reduction patterns shift spatially following the shifting the geographic location of assimilated uncertainty. The uncertainties of the different control variables are dynamically coupled as all change in response to modifying the assimilated uncertainty of one of them.

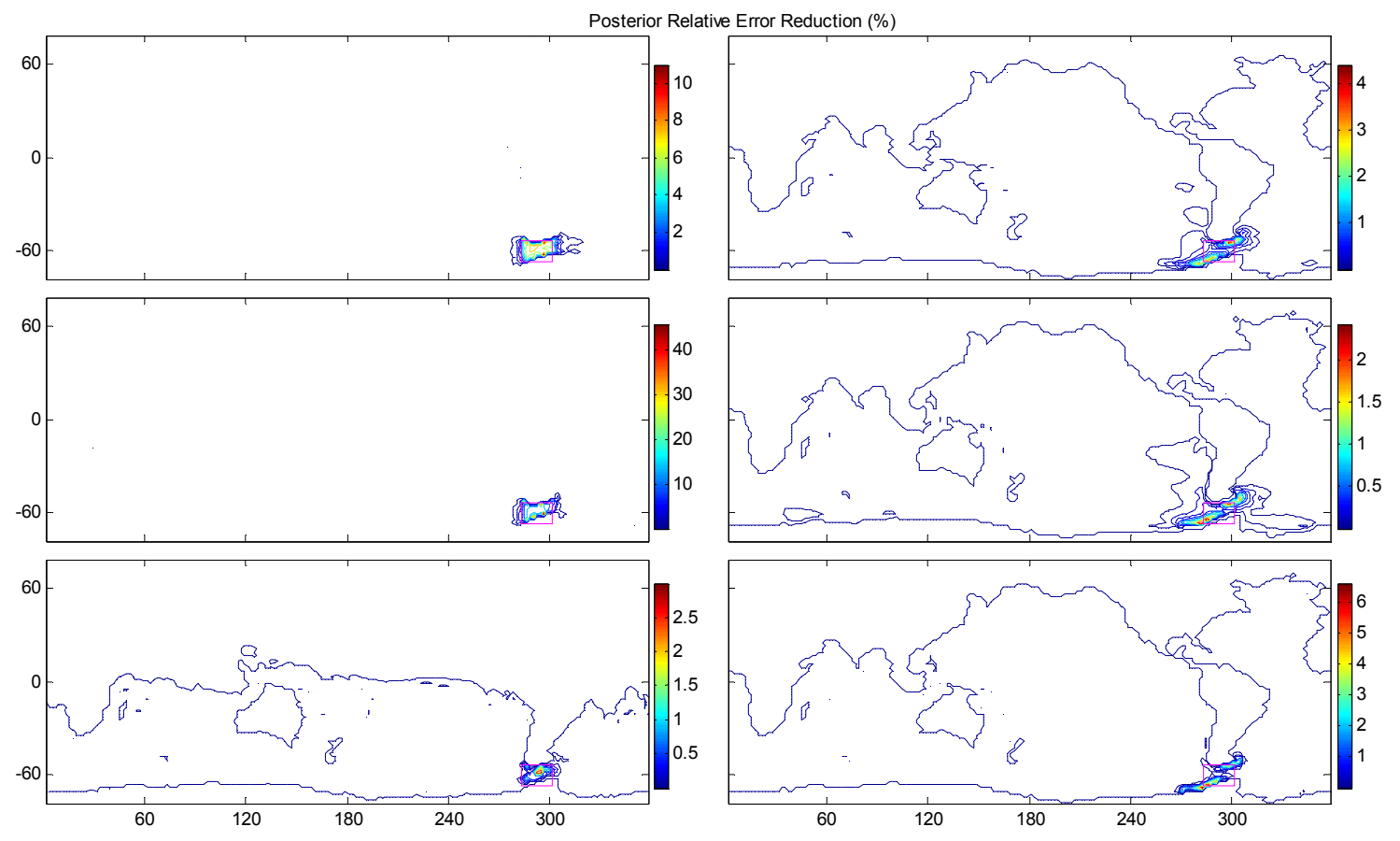

Figure 5.36 Relative uncertainty reduction with Drake array altimetry observations in realistic bathymetry configuration for uncertainty assimilation after 1 day. The prior uncertainties are given by $\left[\Delta \tau^{\mathrm{x}}, \Delta \tau^{\mathrm{y}}, \Delta r\right.$, $\left.\Delta u_{t=0,} \quad \Delta v_{t=0,} \quad \Delta \eta_{t=0}\right]^{T}=[1 \mathrm{~Pa}, 1 \mathrm{~Pa}, 5.0 \mathrm{e}-3 \mathrm{~m} / \mathrm{s}, 0.01 \mathrm{~m} / \mathrm{s}, 0.01 \mathrm{~m} / \mathrm{s}, 0.1 \mathrm{~m}]^{T}$. The location of the assimilated observations is marked by the magenta rectangle, here shown on all panels. 


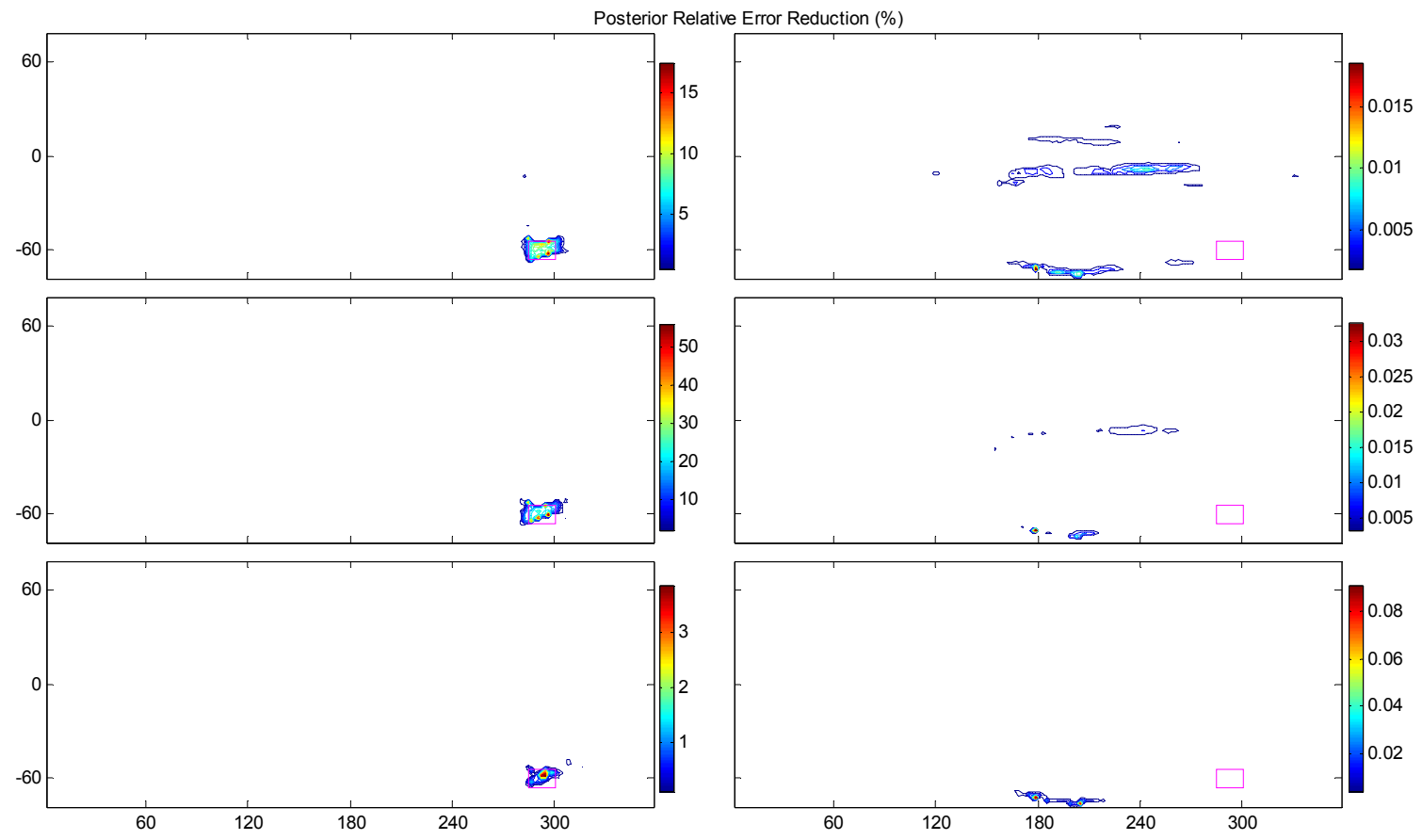

Figure 5.37 Same as Figure 5.36 but for 10 days uncertainty assimilation time.

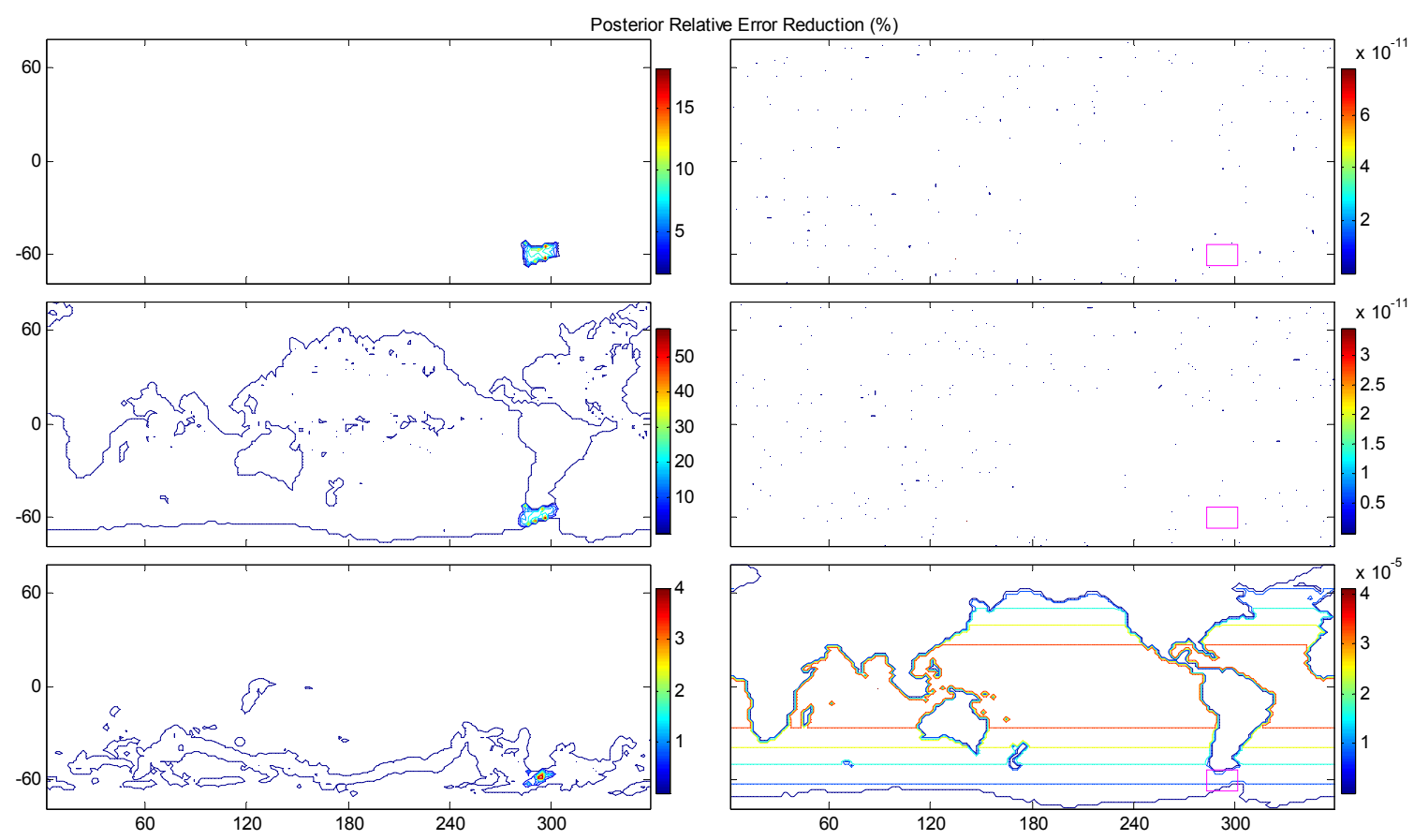

Figure 5.38 Same as Figure 5.36 but for 90 days uncertainty assimilation time. The location of the assimilated observations is marked by the magenta rectangle, here shown on the three right panels. 

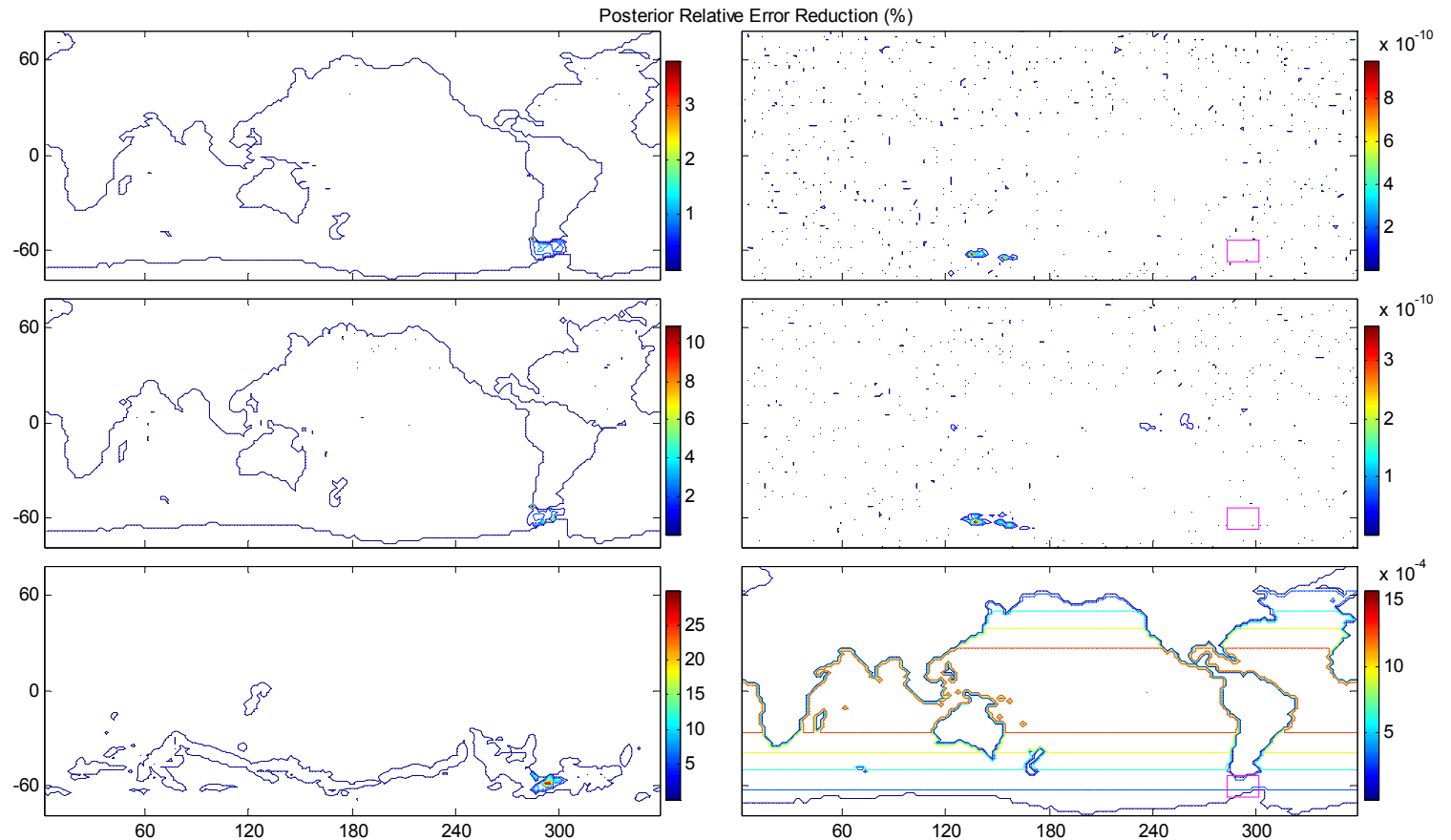

Figure 5.39 Same as Figure 5.38 for 90 days uncertainty assimilation time, but for smaller prior uncertainty of wind stress given by the prior standard errors $\left[\Delta \tau^{\mathrm{x}}, \Delta \tau^{\mathrm{y}}\right]=[0.1 \mathrm{~Pa}, 0.1 \mathrm{~Pa}]$. The location of the assimilated observations is marked by the magenta rectangle, here shown on the three right panels.
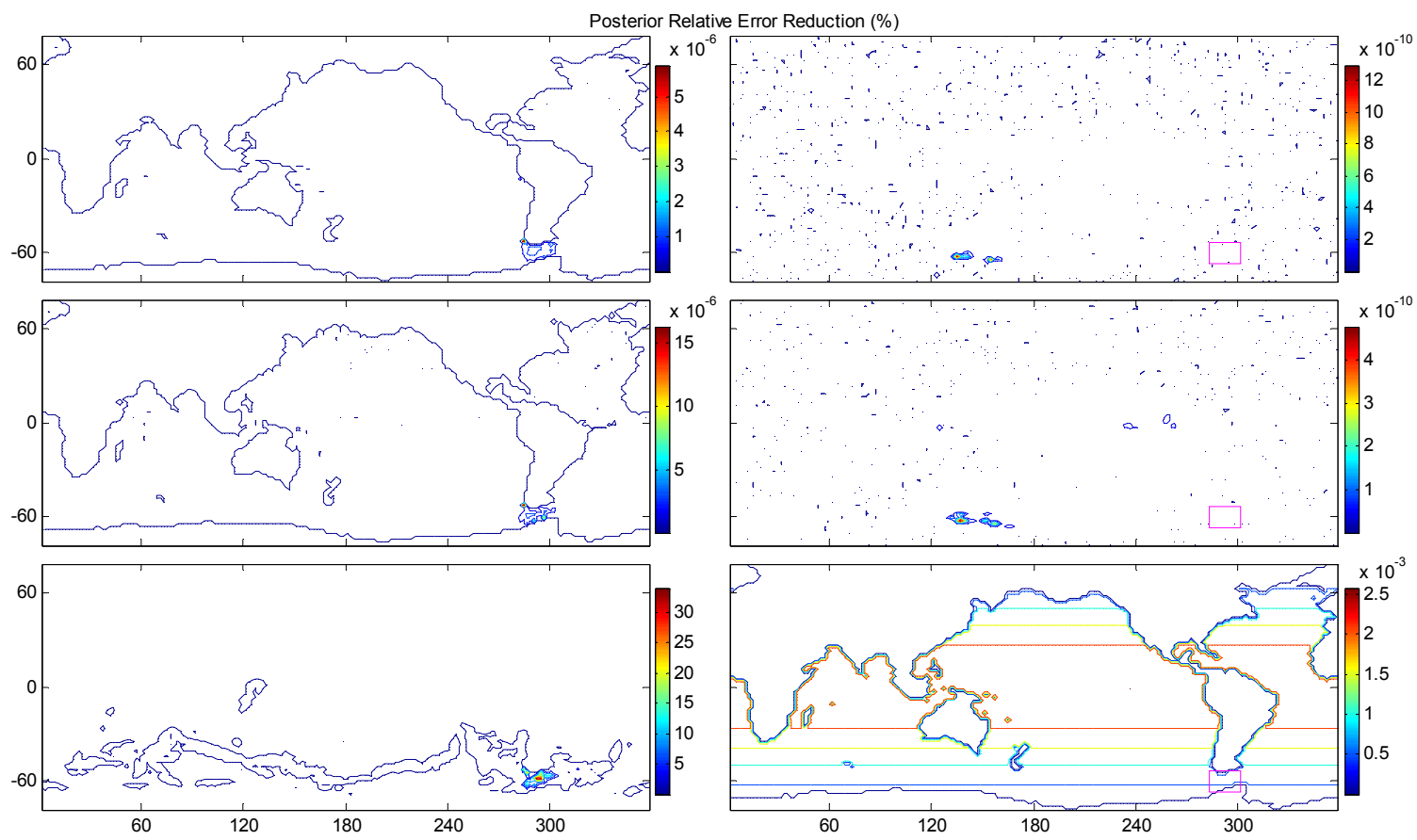

Figure 5.40 Same as Figure 5.38 for 90 days uncertainty assimilation time, but for smaller prior uncertainty of wind stress given by the prior standard errors $\left[\Delta \tau^{\mathrm{x}}, \Delta \tau^{\mathrm{y}}\right]=[0.0001 \mathrm{~Pa}, 0.0001 \mathrm{~Pa}]$. The location of the assimilated observations is marked by the magenta rectangle, here shown on the three right panels. 

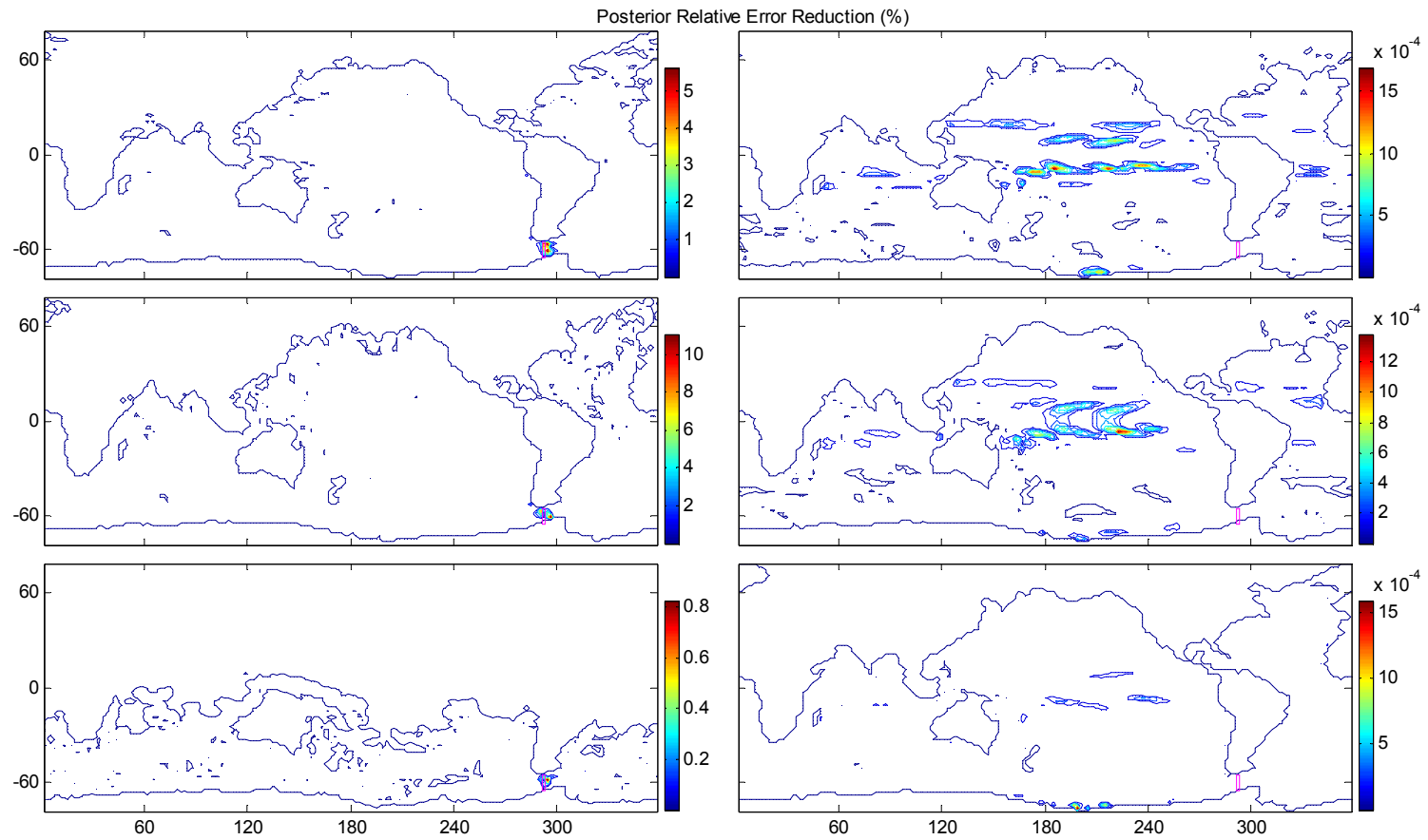

Figure 5.41 Relative uncertainty reduction with Drake transect altimetry observations in realistic bathymetry configuration for uncertainty assimilation after 10 days. The prior uncertainties are same as in Figure 5.36 given by $\left[\Delta \tau^{\mathrm{x}}, \Delta \tau^{\mathrm{y}}, \Delta r, \Delta u_{t=0}, \Delta v_{t=0}, \Delta \eta_{t=0}\right]^{T}=[1 \mathrm{~Pa}, 1 \mathrm{~Pa}, 5.0 \mathrm{e}-3 \mathrm{~m} / \mathrm{s}, 0.01 \mathrm{~m} / \mathrm{s}, 0.01 \mathrm{~m} / \mathrm{s}, 0.1 \mathrm{~m}]^{T}$. The location of the assimilated observations is marked by the magenta rectangle.

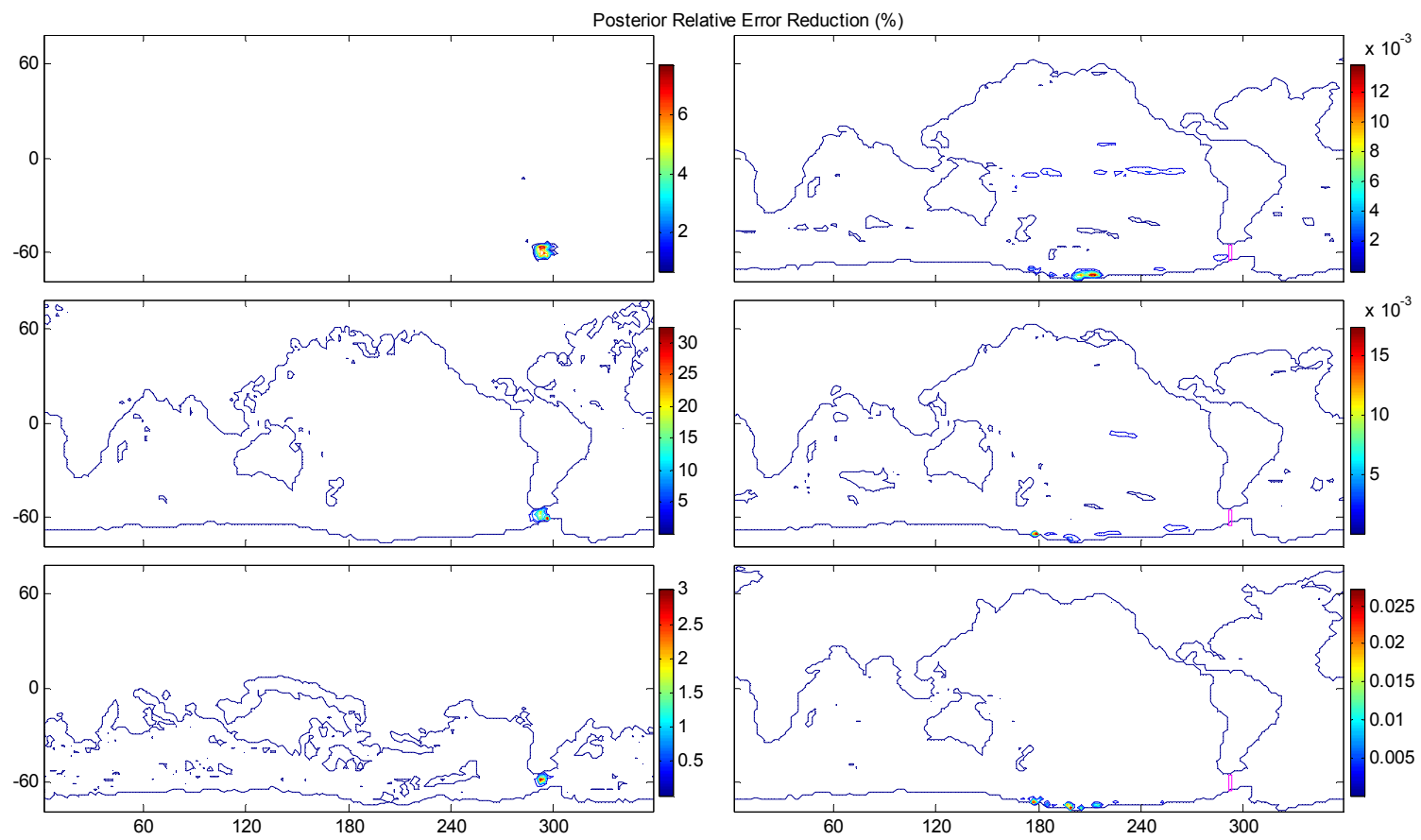

Figure 5.42 Same as Figure 5.41 for 10 days uncertainty assimilation time, but with Drake transect altimetry and currents data. The location of the assimilated observations is marked by the magenta rectangle, here shown on the three right panels. 

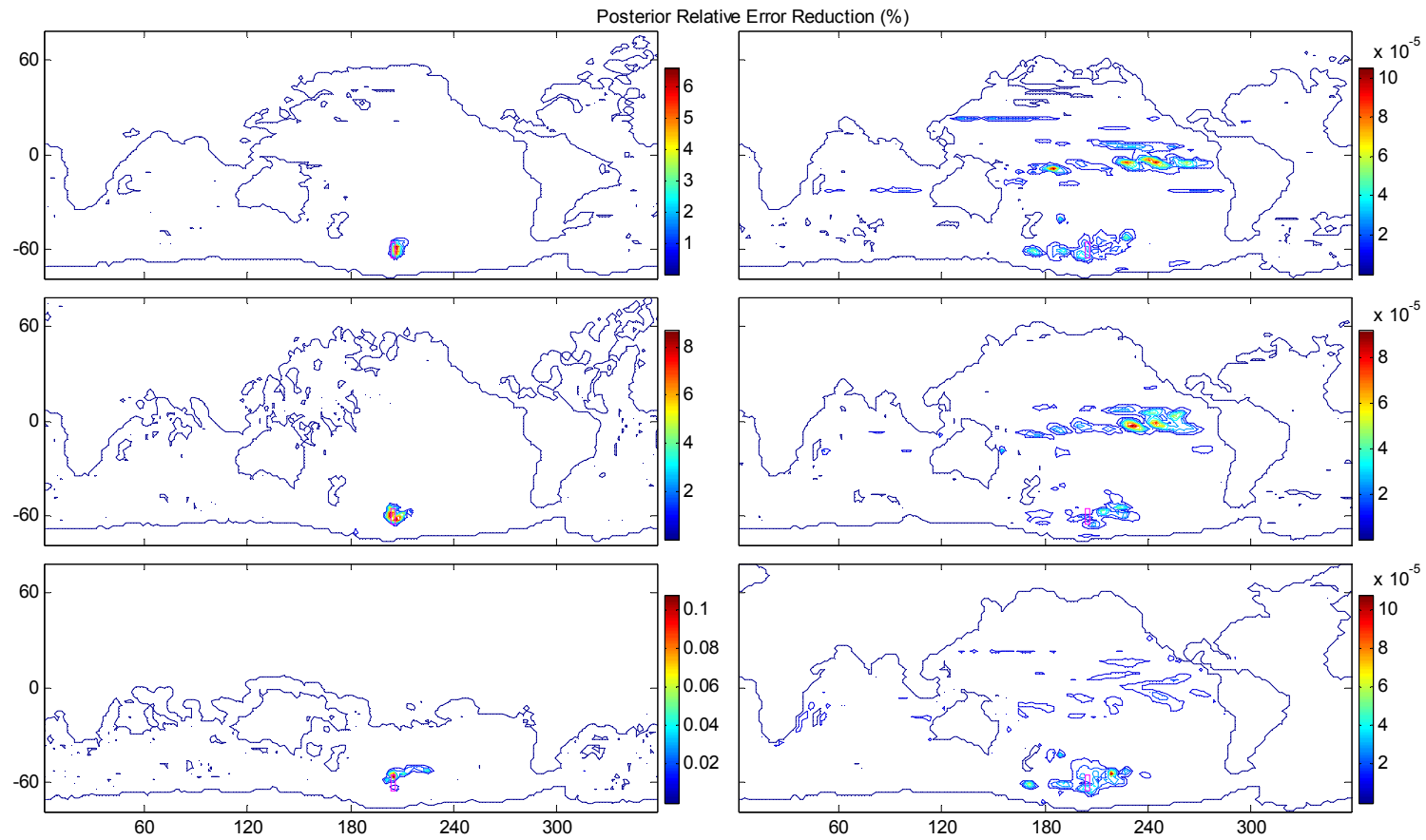

Figure 5.43 Same as Figure 5.41 for 10 days uncertainty assimilation time, but with south Pacific transect altimetry data. The location of the assimilated observations is marked by the magenta rectangle.
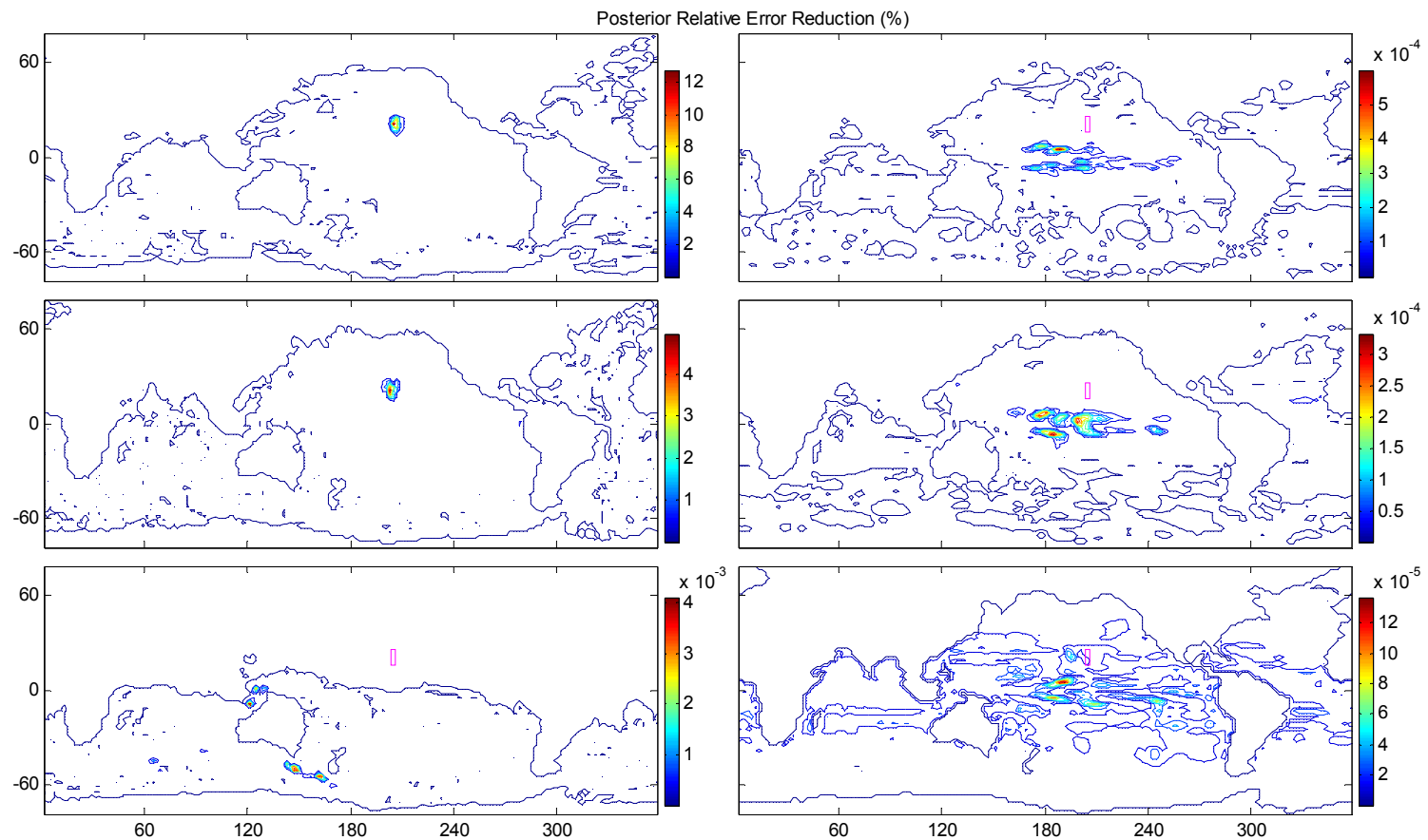

Figure 5.44 Same as Figure 5.41 for 10 days uncertainty assimilation time, but with north Pacific transect altimetry data. The location of the assimilated observations is marked by the magenta rectangle. 


\subsection{Forward uncertainty propagation}

The ultimate goal of the developed inverse-predictive UQ methodology is projection of the assimilated uncertainty to the desired target variables, which in general may be different from one of the model controls. For example, the target quantities can be the circumpolar heat transport or meridional overturning circulation, neither of which is among the model control variables. In this section we present the results of application of the scalable matrix-free algorithm for uncertainty assimilation and forward propagation given by (2.8.3). We propagate the prior and the posterior uncertainties of the controls to the target variable - the Drake Passage transport. Since the transport is not static but a dynamically evolving variable, its uncertainty evolves with time as well. We display the evolution of the prior and the posterior uncertainties of the transport and also calculate the evolution of the relative uncertainty reduction. The resulting timeseries of posterior uncertainty evolution is the formal estimate of the time dependent uncertainty for the time dependent output of ocean state estimate system. It replaces the temporal standard deviation (Figure 1.1) used as fixed uncertainty bounds of the state estimate in previous studies.

Different posterior uncertainty timeseries are calculated for each uncertainty assimilation experiment constructed with different uncertainty assimilation times, different synthetic observation systems (Table 5.1) and different forward model configurations. The results are summarized in the tables below. For simplicity of presentation the tables list the prior and posterior uncertainties only at the time of the assimilated observation data. The following figures add the time dimension to these results.

Before we proceed to describing the results, we illustrate the computational method by displaying the spectral convergence of the uncertainty propagation calculation for spectral truncation of the Hessian of model-data misfit. Figure 5.45 shows the output of the UQ algorithm for posterior uncertainty of the transport calculated with increasing number of Hessian eigenvectors resolved (the reduced Hessian rank). If none are resolved (Hessian rank 0) then the output of the algorithm is the prior uncertainty. If only the leading eigenvector is used (Hessian rank 1) the posterior uncertainty reduction is small. This is an interesting result showing that resolving only one large eigenvalue with the 
leading eigenvector is not enough for this calculation and the converged output is due to the combination of several eigenvalue - eigenvector pairs. This convergence is shown to be achieved successfully with only few leading eigenvectors. The number of the eigenvectors required for convergence can be regarded as the effective rank of the Hessian in the UQ problem. This effective UQ rank (which can be determined by selecting the desired spectral convergence threshold) is equal the dimension of the dominant part of the range of the Hessian and represents the number of independent degrees of freedom constrained in the uncertainty assimilation procedure. The rest of the degrees of freedom in the control space of the ocean model can be regarded as the effective nullspace of the UQ problem. This uncertainty assimilation based partition of the model control space allows separation between the uncertainty due to "errors of commission", which are projected on the range of the Hessian, and the uncertainty due to "errors of omission", which remain unresolved in the nullspace of the UQ problem.
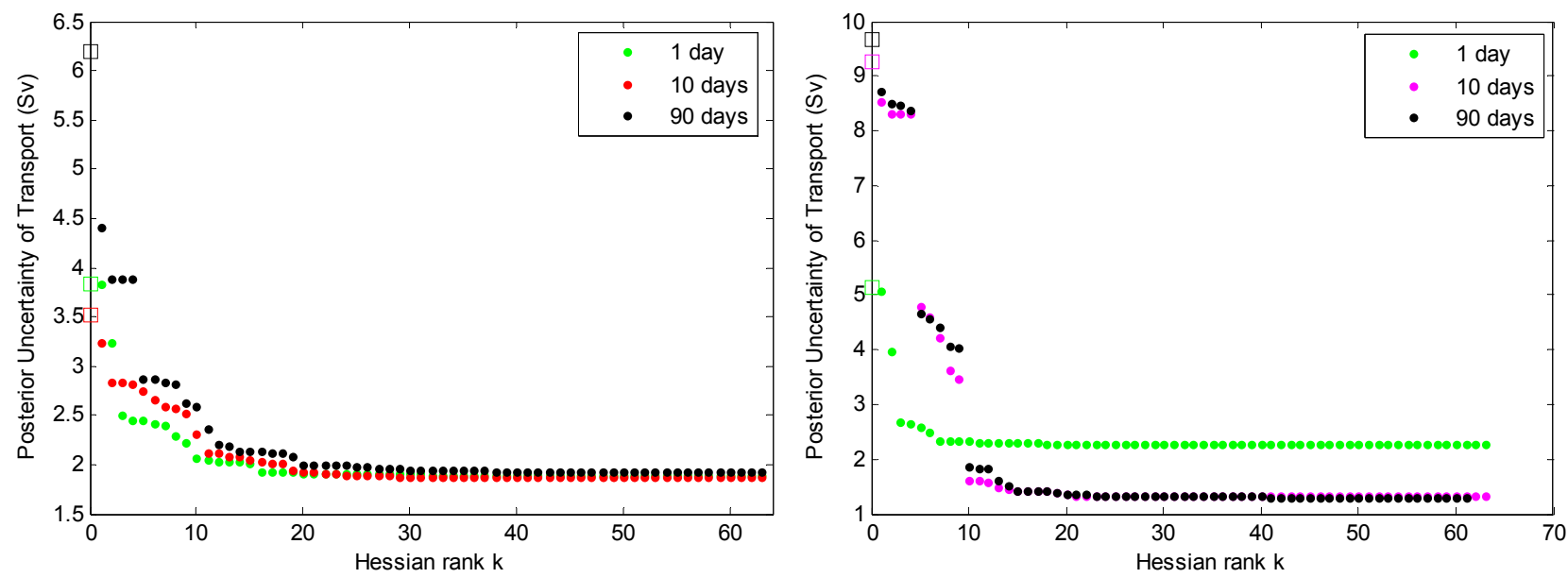

Figure 5.45 Posterior error of Drake Passage transport calculated with increasing Hessian spectral resolution given by the reduced Hessian rank. For zero Hessian rank the result is equal the prior error, shown here with square markers. For increasing Hessian ranks the prior is reduced to monotonously smaller posteriors, converging to the minimum posterior with only few resolved eigenvectors. The left panel is for flat bottom configuration, the right panel is for the realistic bathymetry case.

\subsubsection{Realistic geometry with flat bottom}

Tables 5.2, 5.3 and 5.4 summarize the forward uncertainty propagation results for the flat bottom configuration. The first table compares different uncertainty assimilation times and different observation locations and variables for large prior uncertainty of wind stress. The second table compares the effects of arbitrary selecting different prior uncertainties of wind stress for steady state uncertainty assimilation of Drake Passage 
array altimetry. The third table repeats the first table's experiments with a smaller prior uncertainty of the wind.

Table 5.2 Summary of forward uncertainty propagation experiments for flat bottom configuration with prior uncertainty of controls specified with a diagonal covariance matrix with constant standard errors: $\left[\Delta \tau^{\mathrm{x}}, \Delta \tau^{\mathrm{y}}, \Delta r, \Delta u_{t=0}, \Delta v_{t=0}, \Delta \eta_{t=0}\right]^{T}=[0.1 \mathrm{~Pa}, 0.1 \mathrm{~Pa}, 5.0 \mathrm{e}-3 \mathrm{~m} / \mathrm{s}, 0.01 \mathrm{~m} / \mathrm{s}, 0.01 \mathrm{~m} / \mathrm{s}, 0.1 \mathrm{~m}]^{T}$

\begin{tabular}{|lcccc|}
\hline & $\begin{array}{c}\text { Eigenvalue } \\
1\end{array}$ & $\begin{array}{c}\text { Prior } \\
\text { Uncertainty } \\
(\mathrm{Sv})\end{array}$ & $\begin{array}{c}\text { Posterior } \\
\text { Uncertainty } \\
(\mathrm{Sv})\end{array}$ & $\begin{array}{c}\text { Uncertainty } \\
\text { Reduction } \\
(\%)\end{array}$ \\
\hline 1 day Drake Array Altimetry & $5.55 \mathrm{E}+05$ & 3.842 & 1.899 & 50.6 \\
\hline 10days Drake Array Altimetry & $1.73 \mathrm{E}+06$ & 3.521 & 1.873 & 46.8 \\
\hline 30days Drake Array Altimetry & $3.36 \mathrm{E}+06$ & 5.360 & 1.920 & 64.2 \\
\hline 60days Drake Array Altimetry & $3.87 \mathrm{E}+06$ & 6.080 & 1.922 & 68.4 \\
\hline 90days Drake Array Altimetry & $3.96 \mathrm{E}+06$ & 6.190 & 1.924 & 68.9 \\
\hline 10days Drake Transect Altimetry & $2.74 \mathrm{E}+05$ & 3.521 & 2.672 & 24.1 \\
\hline 10days South Pacific Transect Altimetry & $1.15 \mathrm{E}+05$ & 3.521 & 3.397 & 3.51 \\
\hline 10days North Pacific Transect Altimetry & $8.64 \mathrm{E}+03$ & 3.521 & 3.507 & 0.384 \\
\hline 10days Drake Transect Altimetry \& Flow & $5.26 \mathrm{E}+06$ & 3.521 & 1.332 & 62.2 \\
\hline
\end{tabular}

Table 5.3 Same as Table 5.2 except for different prior uncertainties of wind stress as specified.

\begin{tabular}{|lcccc|}
\hline & $\begin{array}{c}\text { Eigenvalue } \\
1\end{array}$ & $\begin{array}{c}\text { Prior } \\
\text { Uncertainty } \\
(\mathrm{Sv})\end{array}$ & $\begin{array}{c}\text { Posterior } \\
\text { Uncertainty } \\
(\mathrm{Sv})\end{array}$ & $\begin{array}{c}\text { Uncertainty } \\
\text { Reduction } \\
(\%)\end{array}$ \\
\hline $\begin{array}{l}\text { 90days Drake Array Altimetry, } \\
\text { Prior } \Delta \tau=0.1 \mathrm{~Pa}\end{array}$ & $3.96 \mathrm{E}+06$ & 6.190 & 1.924 & 68.9 \\
\hline $\begin{array}{l}\text { 90days Drake Array Altimetry, } \\
\text { Prior } \Delta \tau=0.01 \mathrm{~Pa}\end{array}$ & $3.96 \mathrm{E}+06$ & 3.488 & 1.139 & 67.4 \\
\hline $\begin{array}{l}\text { 90days Drake Array Altimetry, } \\
\text { Prior } \Delta \tau=0.0001 \mathrm{~Pa}\end{array}$ & $3.96 \mathrm{E}+06$ & 3.450 & 1.121 & 67.5 \\
\hline
\end{tabular}

Table 5.4 Same as Table 5.2 for flat bottom configuration for prior uncertainty of controls with standard errors: $\left[\Delta \tau^{\mathrm{x}}, \Delta \tau^{\mathrm{y}}, \Delta r, \Delta u_{t=0}, \Delta v_{t=0}, \Delta \eta_{t=0}\right]^{T}=[0.0001 \mathrm{~Pa}, 0.0001 \mathrm{~Pa}, 5.0 \mathrm{e}-3 \mathrm{~m} / \mathrm{s}, 0.01 \mathrm{~m} / \mathrm{s}, 0.01 \mathrm{~m} / \mathrm{s}, 0.1 \mathrm{~m}]^{T}$.

\begin{tabular}{|lcccc|}
\hline & $\begin{array}{c}\text { Eigenvalue } \\
1\end{array}$ & $\begin{array}{c}\text { Prior } \\
\text { Uncertainty } \\
(\text { Sv })\end{array}$ & $\begin{array}{c}\text { Posterior } \\
\text { Uncertainty } \\
(\text { Sv })\end{array}$ & $\begin{array}{c}\text { Uncertainty } \\
\text { Reduction } \\
(\%)\end{array}$ \\
\hline 1 day Drake Array Altimetry & $5.55 \mathrm{E}+05$ & 3.809 & 1.885 & 50.5 \\
\hline 10days Drake Array Altimetry & $1.73 \mathrm{E}+06$ & 2.306 & 1.420 & 38.4 \\
\hline 30days Drake Array Altimetry & $3.36 \mathrm{E}+06$ & 2.929 & 1.111 & 62.1 \\
\hline 60days Drake Array Altimetry & $3.87 \mathrm{E}+06$ & 3.378 & 1.117 & 66.9 \\
\hline 90days Drake Array Altimetry & $3.96 \mathrm{E}+06$ & 3.450 & 1.121 & 67.5 \\
\hline 10days Drake Transect Altimetry & $2.74 \mathrm{E}+05$ & 2.306 & 1.909 & 17.2 \\
\hline 10days South Pacific Transect Altimetry & $1.15 \mathrm{E}+05$ & 2.306 & 2.214 & 4.02 \\
\hline 10days North Pacific Transect Altimetry & $8.64 \mathrm{E}+03$ & 2.306 & 2.300 & 0.27 \\
\hline 10days Drake Transect Altimetry \& Flow & $5.26 \mathrm{E}+06$ & 2.306 & 1.209 & 47.6 \\
\hline
\end{tabular}


The figures that follow the tables add the time evolution dimension to the results in the tables. However, that time dependence of the uncertainty results is also reflected in the tables. Looking on Tables 5.2 and 5.4 one can see that by increasing the duration of uncertainty assimilation from 1 day to 10,30,60 and 90 days - the leading eigenvalue increases. This, generally, indicates growth of the Hessian spectrum magnitude associated with the increase of the assimilated information. As Figure 5.19 illustrates, not the entire spectrum grows and the growth of the leading eigenvalue is related to concentration of the assimilated information in the leading eigenmodes of the Hessian.

Comparing the eigenvalues of different 10 days experiments (Tables 5.2 and 5.4) with different assimilated observation systems (Table 5.1), we see that assimilating less data points decreases the assimilated information. Same is true if the assimilated data is moved away from the Drake Passage. The assimilated information for the "Drake Passage Transect Altimetry" is more than double than for the "South Pacific Transect Altimetry" case which is shifted on the same latitude to the middle of South Pacific Ocean. Moving the data to the north Pacific reduces the assimilated information by almost 2 orders of magnitude. Adding the currents data to Drake transect assimilation ("10days Drake Transect Altimetry \& Flow" experiment) raises the eigenvalue by an order of magnitude to more than what even the 2-dimensional altimetry array ("10days Drake Array Altimetry") provides at the steady state.

Note, that the eigenvalues in Table 5.3 are all the same. That table compares the effects of different priors with same uncertainty assimilation experiment. Clearly, different prior selections do not affect the assimilated Hessian information.

\subsubsection{Prior uncertainty propagation}

Projecting uncertainty of controls to the target variable space is a nontrivial calculation, which was not implemented with MITgem before. Forward projection of the prior uncertainty of controls is independent of uncertainty assimilation experiments and is evaluated with the Jacobian derivative code of the target variable, see first row of (2.9.3). Although no Hessian code is involved, the results are complex and reflect the dynamics of the uncertainty in the system. 
The prior estimate of uncertainty of transport is not constant and varies as function of time. Surprisingly, this is true even if the transport itself is constant and does not vary with time, because the system is in the steady state. This apparent paradox is resolved by understanding that the evolution of the ocean system and the evolution of its uncertainty are governed by two separate equations representing mutually dependent and yet different dynamics. By initializing the forward ocean model with initial conditions in the steady state balance, the resulting forward solution remains in the steady state for all time, but its uncertainty evolves due to solution's evolving sensitivity to the initial and boundary conditions uncertainty.

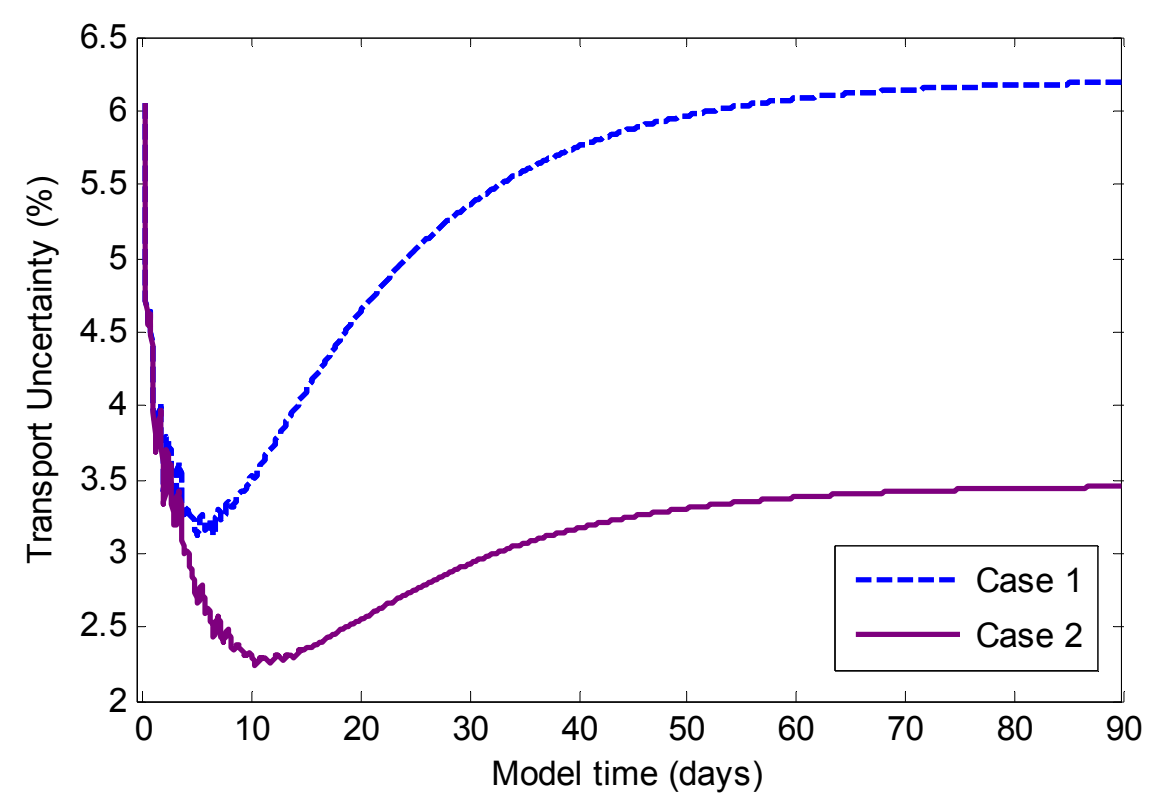

Figure 5.46 Evolution of the prior uncertainties of Drake Passage transport given in percents as fraction of the actual transport at the time of forward uncertainty propagation. Shown here for two cases: the less a priory constrained controls (Table 5.2) and the more certain prior controls case (Table 5.4).

The nonstationary values of the prior transport uncertainty can be read from the second column of tables 5.2 and 5.4, for the two different sets of prior uncertainties of controls. In both cases after one day integration from the steady state, the prior uncertainty of transport is $3.8 \mathrm{~Sv}$. It drops in both cases in the 10th day, although more sharply for more certain prior case (Table 5.4). In the following days the prior uncertainties of transport grow, reaching higher for the less certain case (Table 5.2). More detailed time evolution of these prior transport uncertainties can be seen in Figure 5.46. The prior standard errors of the transport start at $6 \mathrm{~Sv}$ in the first hour of the uncertainty propagation simulation (in this particular simulation the steady state transport is $100 \mathrm{~Sv}$, such that the percentage 
value in the figure is also the absolute value in Sv). Both curves decline together in the first day of the simulation. The uncertainty in the less certain case drops to the minimum $3.2 \mathrm{~Sv}$ after 5 days of forward propagation, and monotonically grows asymptoting to 6.2 Sv after 90 days. The uncertainty of the more certain case drops further to the minimum of $2.3 \mathrm{~Sv}$ after 10 days and grows asymptotically to $3.5 \mathrm{~Sv}$.

Not shown in Figure 5.46 is the initial value of prior transport uncertainty, due to the prior uncertainty of the initial conditions - independent of the dynamics of the ocean. That initial transport uncertainty can be calculated as following from the assumed prior uncertainty of the initial zonal flow $\Delta u_{t=0}=0.01 \mathrm{~m} / \mathrm{s}$. There are 5 grid cells spanning the Drake Passage at the longitude of the transport calculation. The cross sectional area of each westerly face is $a=1.1 \times 10^{9} \mathrm{~m}^{2}$. Since the transport is just the sum of the zonal volume fluxes $z=a \Sigma \mathrm{u}_{\mathrm{i}}$, and if the uncertainties of the initial zonal flow are independent, then the uncertainty of the transport is given by addition in quadrature of each of the grid cell contributions. The value of the initial prior uncertainty of zonal transport is $24.6 \mathrm{~Sv}$, which is much larger than the uncertainty propagated by the ocean model.

We learn that transport uncertainties estimated in the dynamical ocean model are substantially smaller than the static uncertainties due to independent "measurements" at each grid cell. The explanation for this is that the sensitivity of the transport to the uncertainties in model initial and boundary conditions are much weaker than the sensitivity to the flow at the Passage at the moment of transport calculation. The latter is given by the vertical cross sectional area of each grid cell $a=1.1 \times 10^{9} \mathrm{~Sv} \cdot 10^{-6} /(\mathrm{m} / \mathrm{s})$, while a representative example of the former is the sensitivity of transport to the initial zonal flow, which 2 hours after model initialization is $O\left(5 \times 10^{7}\right) \mathrm{Sv} \cdot 10^{-6} /(\mathrm{m} / \mathrm{s})$ (see Figure 5.9).

It is important to highlight that the presented apparent uncertainty reduction in the ocean model is not due to constraint of model uncertainty by assimilated observations. The calculations shown are for prior transport uncertainty only, based on assumption of prior uncertainty of ocean model controls. The reduced uncertainty in the model may reflect an incomplete resolution of all the uncertainty factors contributing to the real uncertainty of transport. The uncertainty resolved in the ocean model is only a measure of uncertainty due to "error of commission", any uncertainty which is not represented in the modeling 
framework is not reflected in our calculations and should be considered as a possible "error of omission". Including the uncertainties due to "errors of omission" is a grand challenge in model based UQ and is not considered further in this dissertation.

The resolved evolution of the forward projected prior uncertainty (Figure 5.46) can be understood further by a detailed analysis of the forward propagation operator (2.3.11). For the specified diagonal prior (5.2.1), i.e. without correlations of control uncertainties, the forward projection reduces to summation of control variances weighted by the sensitivities of the transport:

$$
\sigma_{z}^{2}=\sum_{i}\left(\frac{\partial N}{\partial x_{i}}\right)^{2} \sigma_{x_{i}}^{2}
$$

This summation is known as uncertainty addition in quadrature (Taylor 1982). For each forward model time, the vector of the uncorrelated contributions to the variance of the transport can be plotted by multiplying sensitivity maps with the prior uncertainties of controls and squaring the result. Equivalently, the absolute values of the uncertainty weighted sensitivity maps can be shown. Closely related maps were analyzed by Heimbach et al. (2011) by multiplication of sensitivities with uncertainties based on the observed variability of ocean fields (Forget and Wunsch 2007). The difference is that in that study the absolute value was not taken and the "normalized sensitivities" were treated as "normalized response fields" to expected uncertainties in observations. Here, we extend that uncertainty analysis approach by formal application of the quadrature rule.

Two uncertainty maps of independent uncertainty contributions to the prior uncertainty of Drake Passage transport evolution are shown in Figure 5.47 and Figure 5.48. Both uncertainty contribution snapshots are shown for Case 1 of Figure 5.46, one after 6 hours from uncertainty model initialization and one after 10 days. The physical mechanisms of the uncertainty contributions can be inferred from the spatial patterns and the time dependent evolution of the uncertainty fields. After 6 hours the dominant uncertainty contribution is due to the adjoint Kelvin waves propagating equatorward along the western American coast and eastward along the Antarctic continent. After 10 days the contribution due to the adjoint Rossby waves are clearly seen in the meridional velocity uncertainty panel. That uncertainty, however, is not the dominant one. Locally large uncertainty values are due to the boundary conditions - wind forcing and bottom friction 

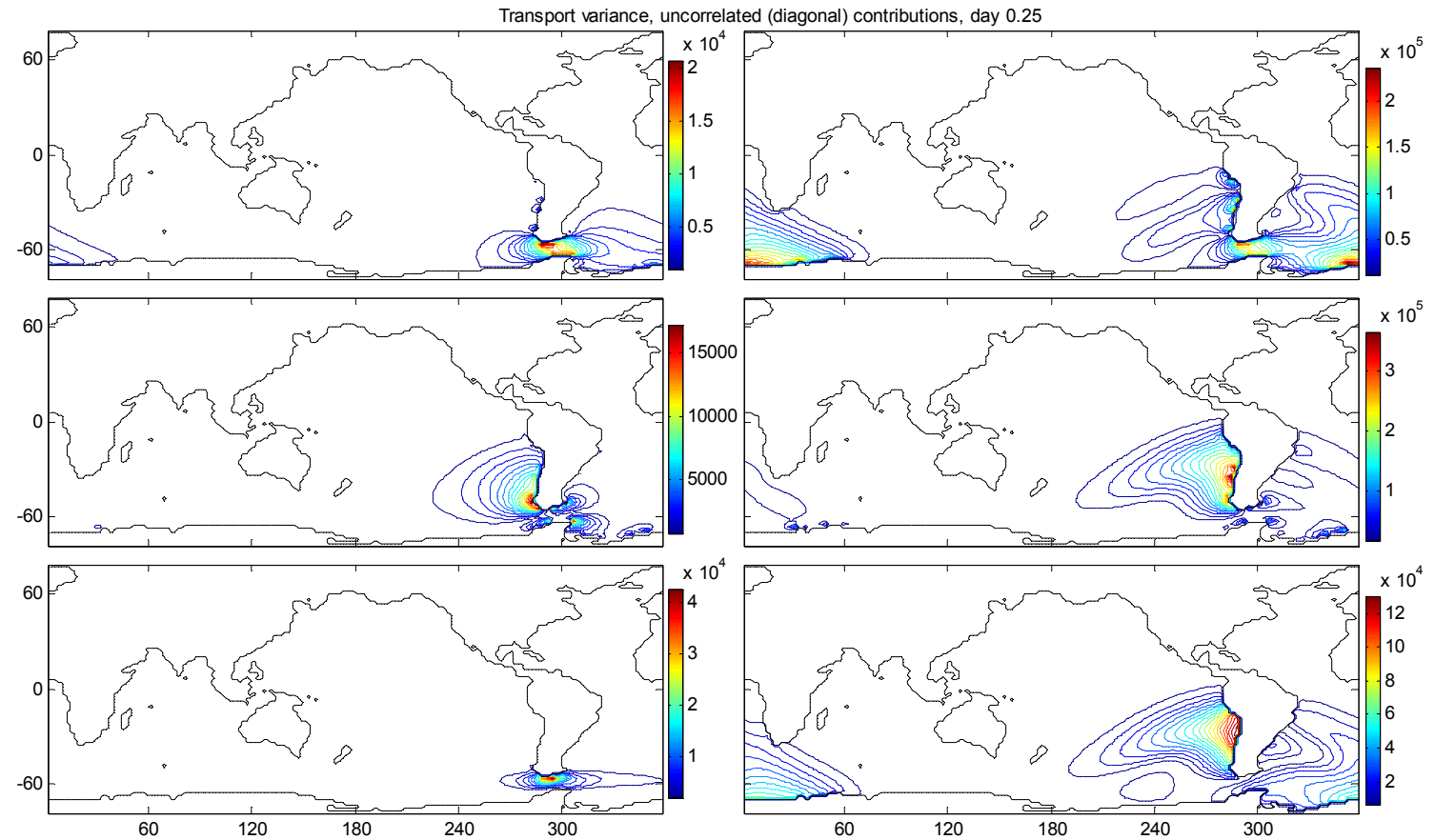

Figure 5.47 Map of prior Drake Passage transport uncertainty contributions due to control fields, in volume transport units $\left(\mathrm{m}^{3} / \mathrm{s}\right)$. Shown for the prior transport uncertainty 6 hours after uncertainty propagation model initialization for the prior control parameters as in Table 5.2.

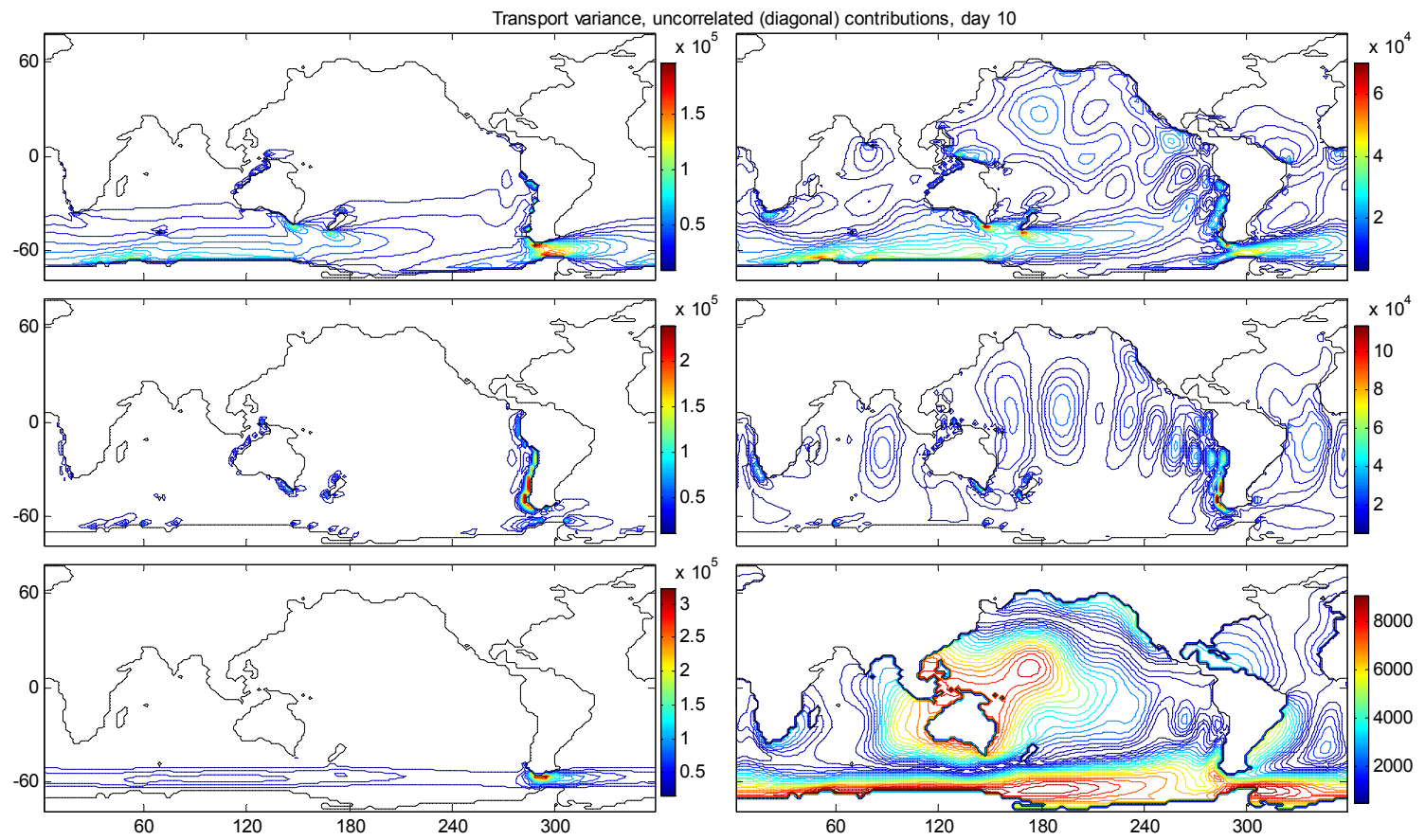

Figure 5.48 Same as in Figure 5.47, shown for the prior transport uncertainty 10days after uncertainty propagation model initialization 
uncertainty fields. Considering the integral contribution over the whole domain, the zonal wind forcing uncertainty appears to dominate. This can be confirmed quantitatively by plotting the quadrature integrals over each panel in Figure 5.49. The plot is the partition of the prior uncertainty evolution curve for Case 1 in Figure 5.46 to 6 separate control fields uncertainty contributions. The uncertainty due to the initial conditions dominate in the first 5 days, exponentially decaying to zero at the steady state as the system "forgets" the initial conditions. The uncertainty due to the boundary conditions grow from zero to their steady state values on the time scale of frictional relaxation of the ACC. The dominant uncertainty source in the steady state is zonal wind forcing. The second strongest uncertainty source is the bottom friction field.

These detailed quantitative results are consistent with the conclusions based on the idealized analytical ODE uncertainty quantification analysis - the effects of initial conditions decay exponentially, while forcing and friction effects grow with exponential relaxation to the steady state values. The developed here forward uncertainty propagation analysis approach allows a detailed quantitative understanding of transport uncertainty sources, their evolution and a qualitative identification of their physical mechanisms.

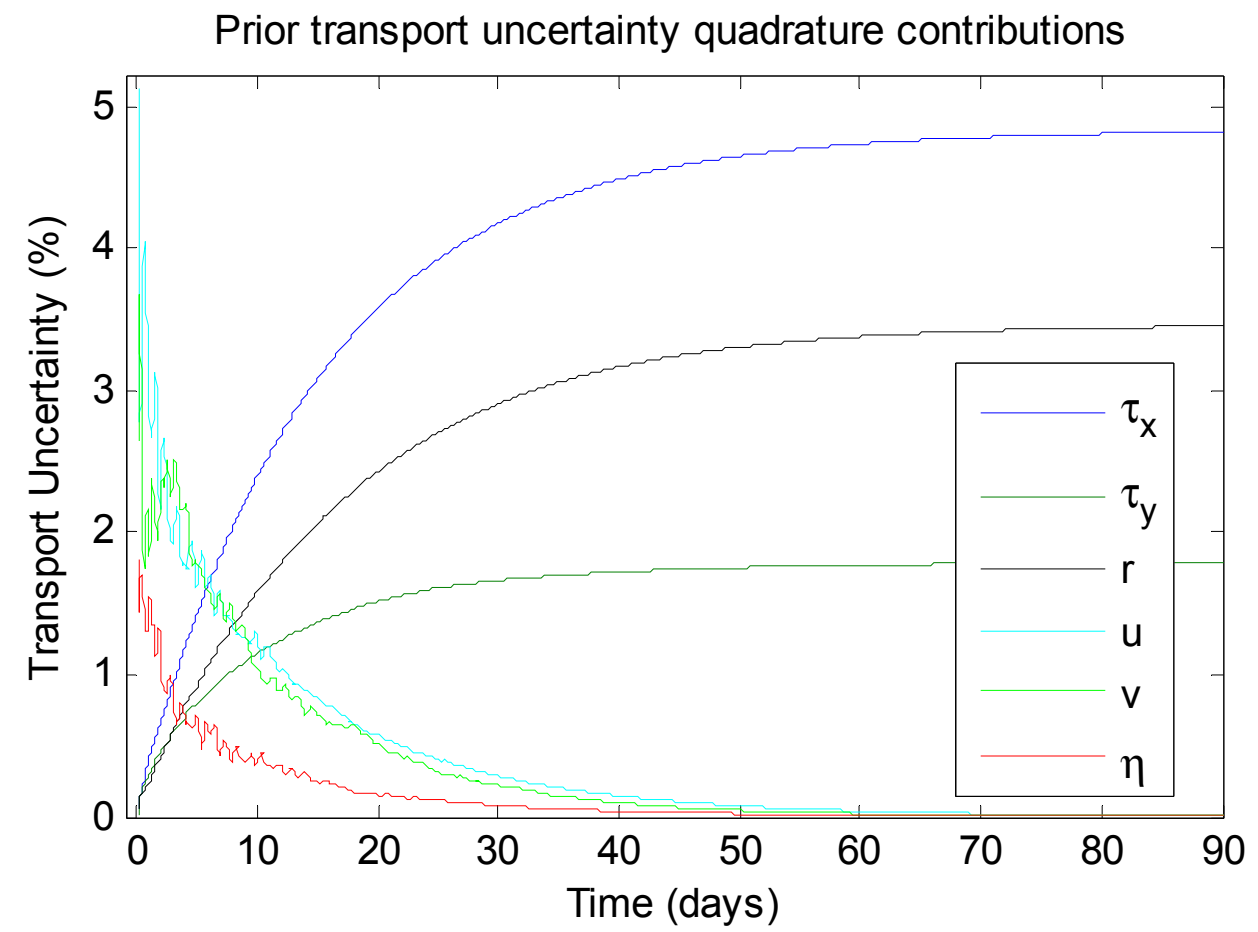

Figure 5.49 Uncertainty variance contributions due to each of six control fields to the prior transport uncertainty evolution for the prior control parameters as in Table 5.2. Shown in percents of the total variance of the prior transport uncertainty. 


\subsubsection{Posterior uncertainty and uncertainty reduction}

The posterior uncertainty and the relative uncertainty reduction values for the Drake Passage transport (5.2.2) are tabulated in the last two columns of Tables 5.2, 5.3 and 5.4 for the exact time when observations and their uncertainties are assimilated in the model. The posterior uncertainty after 24 hours of model integration, at the time of Drake array altimetry uncertainty assimilation, is reduced by $50.6 \%$. If uncertainty is assimilated after 10 days, the posterior is reduced by $46.8 \%$ at this time. Uncertainty reduction increases for longer data assimilation times when examined at the time of uncertainty assimilation. Just by looking at the table it is not obvious if the increase is due to longer assimilation times or because the system integrates to a steady state equilibrium. This question will be resolved by consideration of the detailed time evolution of the uncertainties in the sections below.

Now, we consider the effects of the different observation systems assimilated 10 days after model initialization. The effects of 10th day uncertainty assimilation on posterior uncertainty reduction are consistent with the trends of the assimilated Hessian information, as discussed in terms of the Hessian eigenvalues above. Restricting assimilated data to one row of altimetry points decreased uncertainty reduction to $24.1 \%$ and the resulting posterior uncertainty is larger, that is $2.7 \mathrm{~Sv}$ vs. $1.9 \mathrm{~Sv}$ for Drake array. Assimilation of uncertainty far in South Pacific leads to much smaller uncertainty reduction, while data uncertainty in the North Pacific almost does not affect the uncertainty of Drake Passage transport. The addition of current meters to Drake transect assimilation constrains more strongly the transport, by $62.2 \%$ compared to $24.1 \%$ for Drake array altimetry only.

\subsubsection{Transport uncertainty constraint by different prior uncertainties of wind forcing}

Considering the effects of different priors in Table 5.3 we see that an order of magnitude smaller prior uncertainties of wind stress result in twice smaller prior uncertainties of the transport (column 2 in the table). That quantifies how much the knowledge of wind stress constrains the uncertainty of predicted Drake transport, which is a valuable UQ result in forward uncertainty propagation framework (unrelated to uncertainty assimilation

problem and the developed inverse propagation machinery). This uncertainty constraint 
effect has a limit, as reducing the prior by two more orders of magnitude does not farther reduce the prior transport uncertainty by much. This indicates that other variables control the remaining uncertainty of the transport, as was explicitly shown in Figure 5.49, and the way to farther reduce this uncertainty is constraining the controls by assimilation of observations. Calculating that in the 3rd column of Table 5.3, we see that the prior uncertainty can be reduced by more than two thirds with assimilation of Drake array altimetry uncertainty. The exact fraction of uncertainty reduction (column 4) decreases by very little if smaller prior uncertainty is assumed. This is confirmed for all the other uncertainty assimilation experiments in Table 5.4, except for the 10 days south Pacific altimetry, where the uncertainty reduction fraction is larger than in Table 5.2, 4\% vs. $3.5 \%$. This means that if wind stresses are better known over the globe, then adding knowledge of ocean surface elevation upstream of the Passage is more valuable for estimating Drake transport than knowledge of the elevation at the Passage itself.

\subsubsection{Time-resolving analysis of uncertainty dynamics}

As mentioned above, the tables present only a partial view of the uncertainty quantification results. The estimated uncertainties vary considerably as function of time and in general are different from those at the exact time of uncertainty assimilation. For example consider the uncertainty reduction due to assimilation of Drake array altimetry 1 day after model initialization (Figure 5.50). It is shown that there is a sharp peak in the posterior uncertainty reduction exactly at the assimilation time, $50.6 \%$ as is listed in Table 5.2, but this peak is not a good representative of the posterior uncertainties and their reduction at the other times. Before and after the uncertainty assimilation moment the posterior uncertainty is only slightly smaller than the prior - i.e. only small uncertainty reduction, asymptoting $1 \%$ reduction in the steady state.

In contrast, assimilation of same altimetry data on 30th day of the steady state simulation (Figure 5.51) produces quite different uncertainty reduction effects. The reduction in the posterior transport uncertainty at the assimilation time is $64.2 \%$, as is listed in Table 5.2. This reduction is not transient and keeps growing slightly to the steady state value. The posterior uncertainty curve drops significantly beneath the prior uncertainty curve in the first 10 days and when the prior uncertainty grows back, the posterior remains low 
growing only a little to its steady state value $2.2 \mathrm{~Sv}$. We conclude that uncertainty varies considerably not only as function of forward uncertainty propagation time, but also as function of the uncertainty inverse propagation time. In this example, the longer inverse propagation time, i.e. the uncertainty assimilation period, resulted in stronger effect on Drake Passage transport uncertainty.
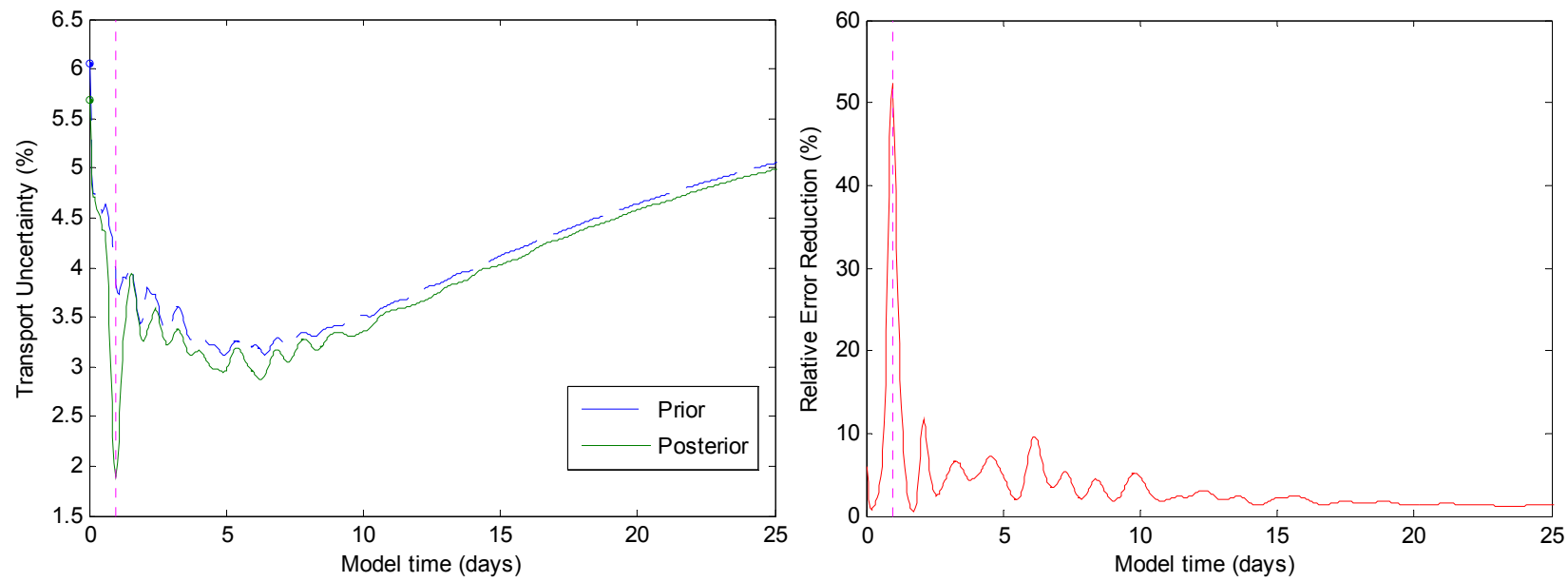

Figure 5.50 Evolution of prior and posterior uncertainties of Drake Passage transport given in percents as fraction of the actual transport at the presented time. Here shown for 1 day Drake array altimetry uncertainty assimilation experiment for flat bottom configuration model. The prior uncertainties are given

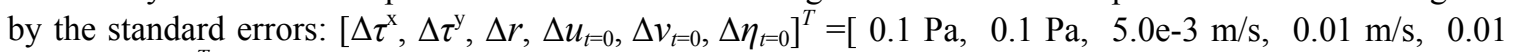
$\mathrm{m} / \mathrm{s}, \quad 0.1 \mathrm{~m}]^{T}$. The left panel compares the prior uncertainty (blue dashed) and the posterior uncertainty (green solid) evolutions. The moment of uncertainty assimilation, here 1 day, is shown by the vertical magenta dotted line. The right panel shows the relative uncertainty reduction fraction in percents (red solid) and a the time of uncertainty assimilation (vertical magenta dotted).

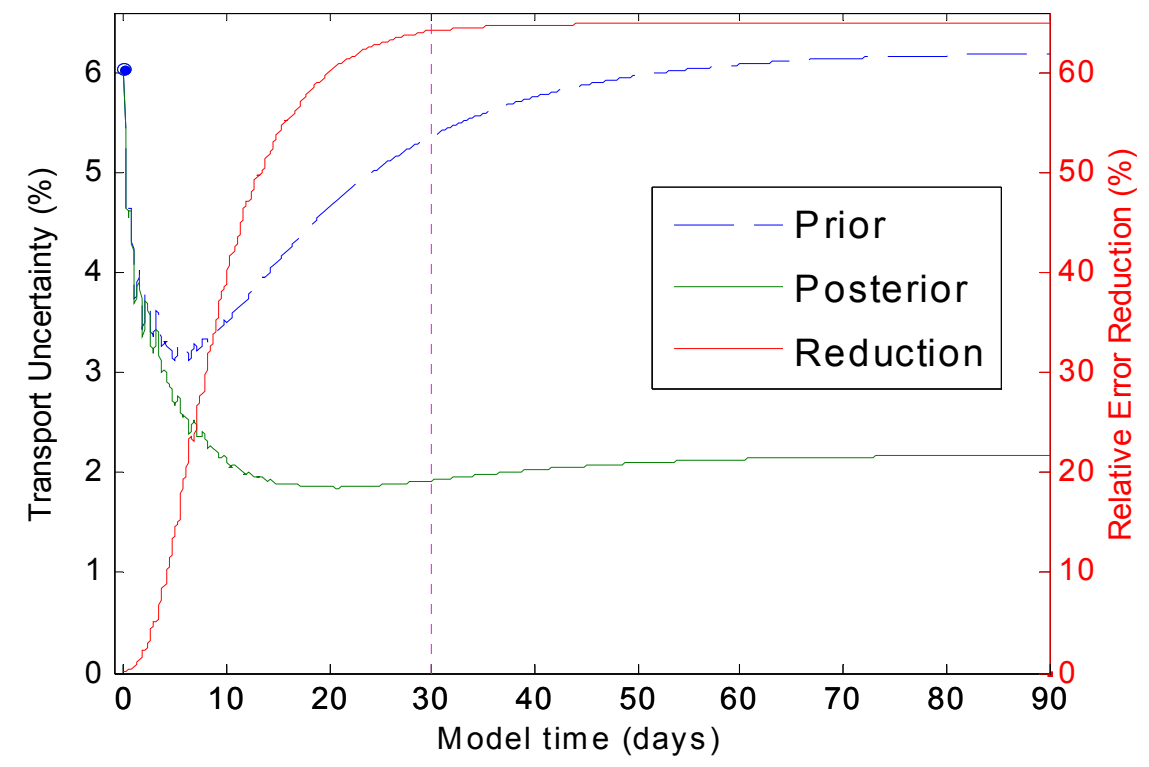

Figure 5.51 Same as in Figure 5.50 but for uncertainty assimilation 30 days after model initialization. For compactness of presentation the two separate panels are combined into single plot. The left-side axis is transport uncertainty in percents, the right-side axis is the uncertainty reduction in percents. 
The striking difference between posterior uncertainty dynamics for different uncertainty assimilation times suggests analyzing in detail the dynamics of uncertainty reduction. In Section 5.3.1.1 a detailed analysis of prior uncertainty evolution and its physical mechanisms was possible by analyzing the separate contributions to the uncertainty of transport given by the quadrature rule. That mapping from uncertainties of controls to the target variable was simplified because the prior control uncertainties were not correlated. This is not the case for the posterior uncertainties of controls, because uncertainty assimilation introduces off-diagonal covariance terms to the posterior control covariance matrix. The posterior mapping from controls to the target variable uncertainty is given by

$$
\sigma_{z}^{2}=\sum_{i}\left(\frac{\partial N}{\partial x_{i}}\right)^{2} \sigma_{x_{i}}^{2}+\sum_{i} \sum_{j \neq i} \frac{\partial N}{\partial x_{i}} \frac{\partial N}{\partial x_{j}} \sigma_{x_{i}} \sigma_{x_{j}} \rho_{i j}
$$

Only the first sum (in quadrature) of sensitivity weighted uncertainties was considered in the previous analysis. One could visualize the spatial structures of the separate control fields contributions for each forward propagation time, or one could plot the time series of the evolution of the separate contributions. It is much more technically challenging to apply similarly detailed analysis to the covariance contributions to the target uncertainty. The double summation includes cross products of sensitivity fields weighted by the covariances of controls, or equivalently, the cross products of sensitivity-weighted posterior uncertainties scaled also by their correlation coefficients. The number of the terms in the double summation is square of the number of quadrature sum terms and therefore cannot be simply visualized for the large dimensionality of the ocean state estimation problem.

The posterior quadrature contribution patterns can be plotted, just as in Section 5.3.1.1, however they are not shown here because visually they are almost indistinguishable from the prior quadrature uncertainties (e.g. Figure 5.47). This can be explained by the relatively small reduction of the diagonal terms in the posterior covariance matrix of controls. The reduction of the uncorrelated part of posterior uncertainty of controls was analyzed in detail in Section 5.2.3. It was shown to reflect spatially meaningful patterns, mostly localized at the assimilated observations regions, but the magnitude of uncertainty reduction was only few percents and less. In fact, the total effect of the reduced variance of controls on the posterior transport uncertainty is very small, as shown in Figure 5.52 
and Figure 5.53 for the day 1 uncertainty assimilation experiment of Figure 5.50. The posterior variance of transport due the quadrature sum in (5.3.2) is practically indistinguishable from the prior transport variance (Figure 5.52), meaning that there is almost no uncertainty reduction due to the uncorrelated part of the posterior uncertainty of the controls (Figure 5.53). The large 50\% uncertainty reduction is completely due to the correlated posterior covariance terms (Figure 5.53). In other words, the double sum in (5.3.2) is negative, as shown in Figure 5.52. Because the prior covariance of controls is diagonal, all the nonzero off-diagonal terms in the posterior covariance matrix are solely due to the uncertainty assimilation. The interpretation is, that the assimilation of observation uncertainty and its inverse propagation through the model cross-couples the control fields and this coupling is the responsible for the bulk of uncertainty reduction.

Same partition to correlated and uncorrelated posterior uncertainty and uncertainty reduction contributions can be plotted for the day 30 assimilation experiment (Figure 5.51). Again, the posterior variance of transport follows closely the prior variance (Figure 5.54) and almost all the uncertainty reduction is due to the off-diagonal posterior control covariance terms (Figure 5.55). However, in this case the contribution of the diagonal covariance terms is more noticeable, up to $3.4 \%$ of the total transport variance reduction. The double sum in (5.3.2) is again negative for all times (Figure 5.54), however, unlike in Figure 5.52 the effect is not a transient peak but grows to a negative steady sate value.

In summary, the have learned the critical role that posterior controls correlations play in UQ. Not only that the quadrature rule for summation of uncertainty sources cannot be applied, but its effect is completely dominated by the off-diagonal covariance terms. Physically, it is the coupling of ocean fields that reduces the uncertainty in ocean state estimation, relative to a priori uncorrelated uncertainties in specified ocean controls. The ocean system appears to be tightly coupled and the developed uncertainty assimilation methodology allows to resolve these correlations. More detailed analysis of the structure of the off-diagonal covariance fields is expected to reveal the physical mechanisms of coupling, but this remains challenging due to the large dimensionality of the covariance data. We highlight, that this challenge is of careful analysis and physical interpretation kind, and is not limited by the technical properties of the machinery developed in this dissertation. 


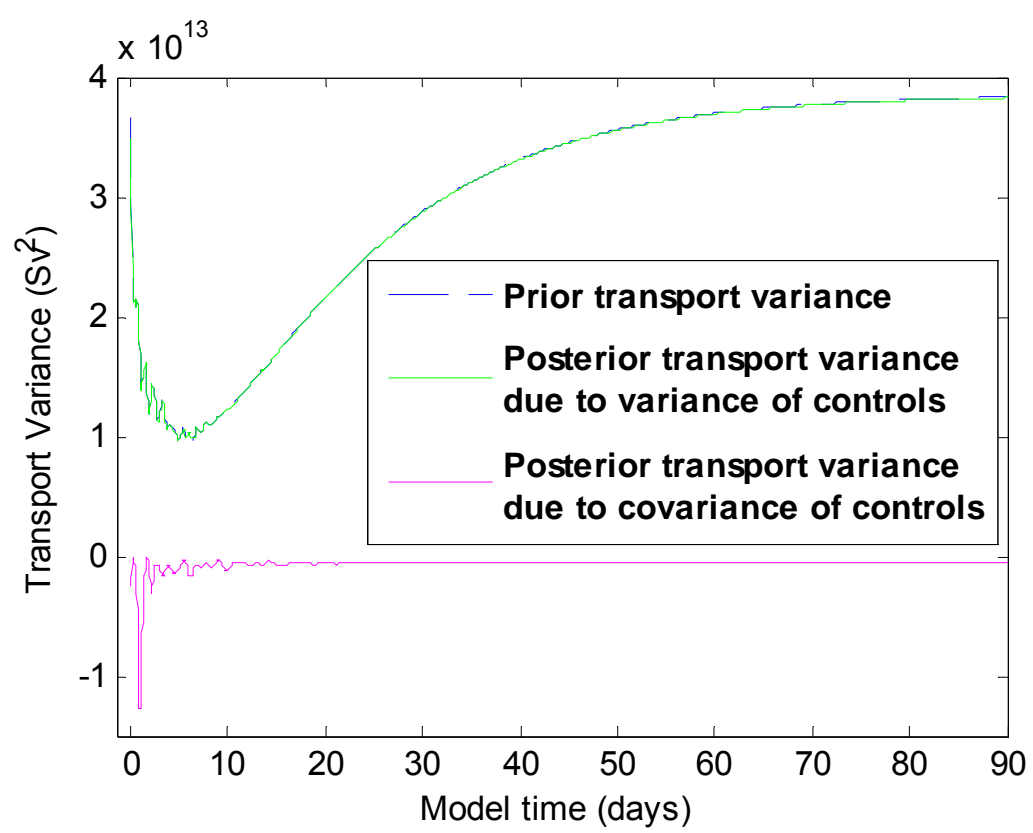

Figure 5.52 Evolution of prior and posterior transport variance for Drake array altimetry assimilation 1 day after model initialization. The posterior uncertainty variance is partitioned to the uncorrelated and the correlated contributions of the posterior uncertainty of the controls.
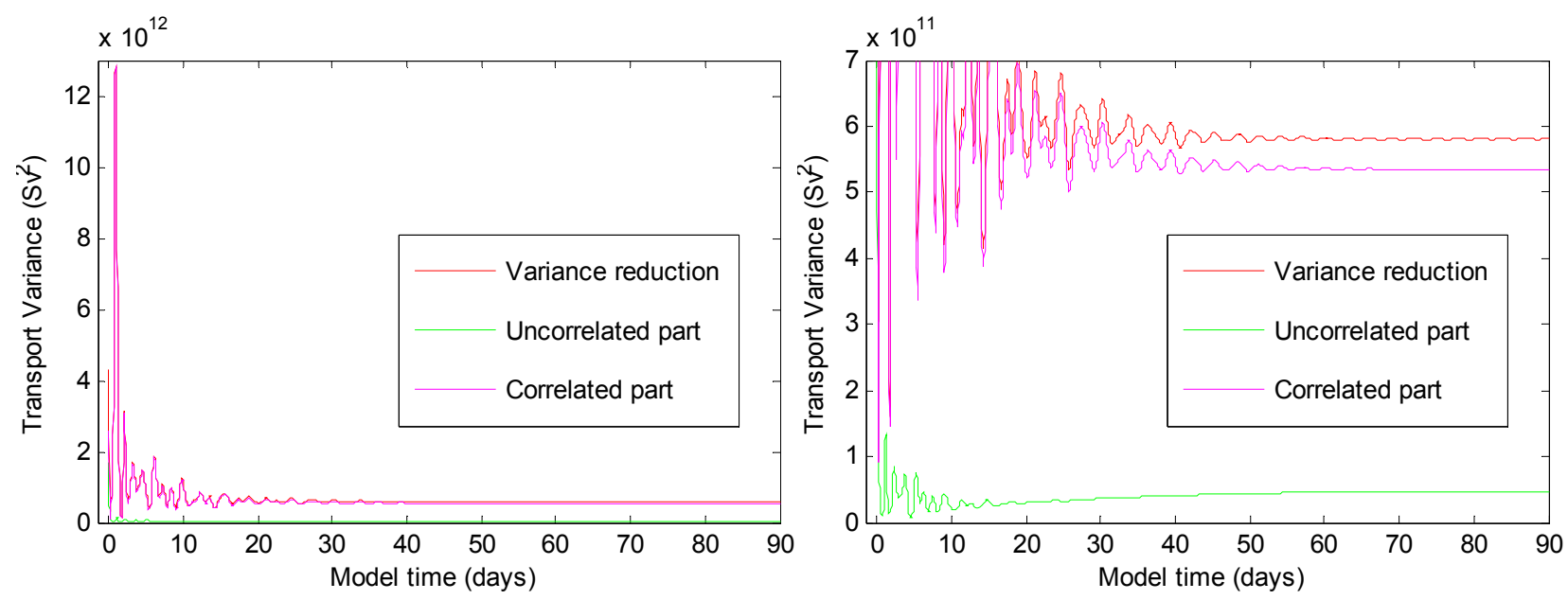

Figure 5.53 Evolution of posterior variance reduction of transport partitioned to reduction due to the diagonal (uncorrelated) and off-diagonal (correlated) parts of the posterior covariance of model controls. Shown for Drake array altimetry assimilation 1 day after model initialization. The right panel shown the same plot with zoomed in vertical axis to discern the miniscule contribution of the uncorrelated part of posterior covariance of controls. Note also the stabilization of uncertainty evolution, which ripples decay in the steady state. 


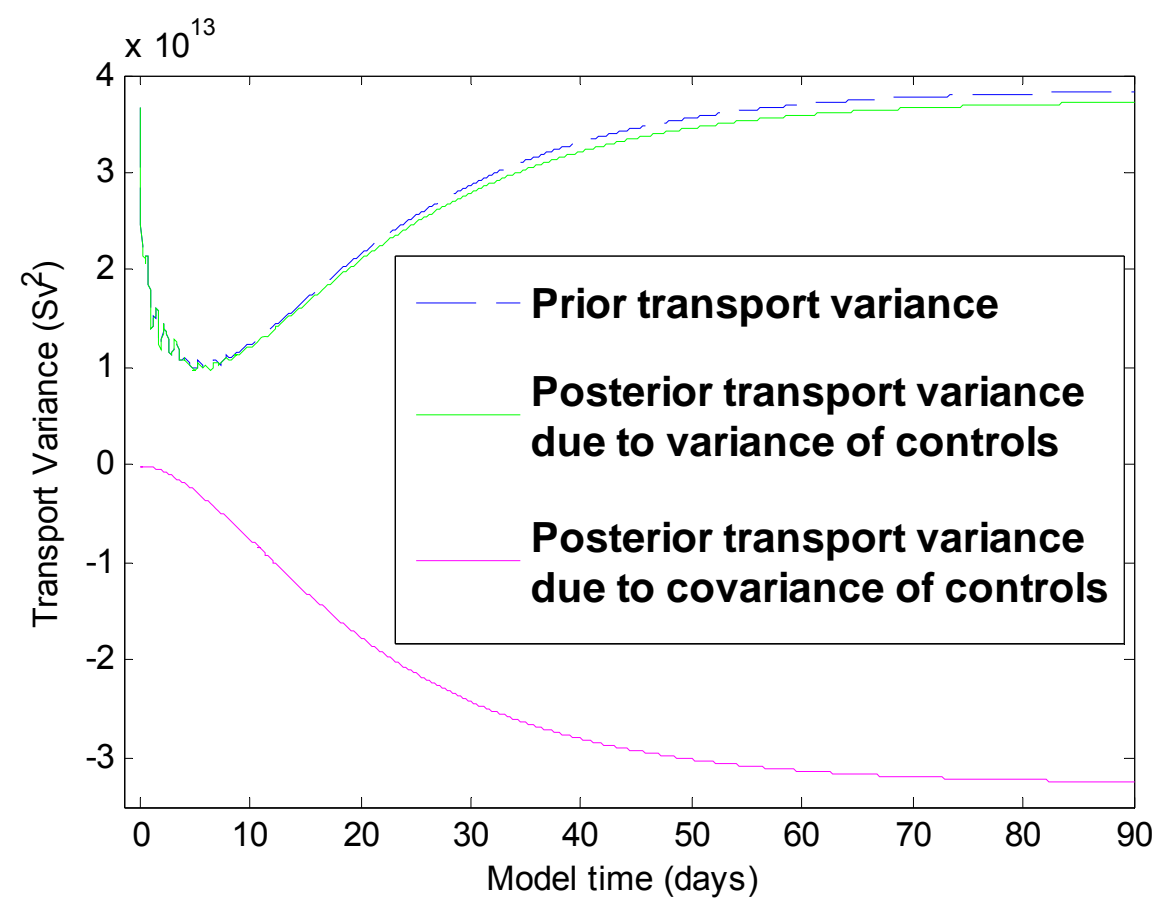

Figure 5.54 Same as Figure 5.52 but for Drake array altimetry assimilation 10 days after model initialization.

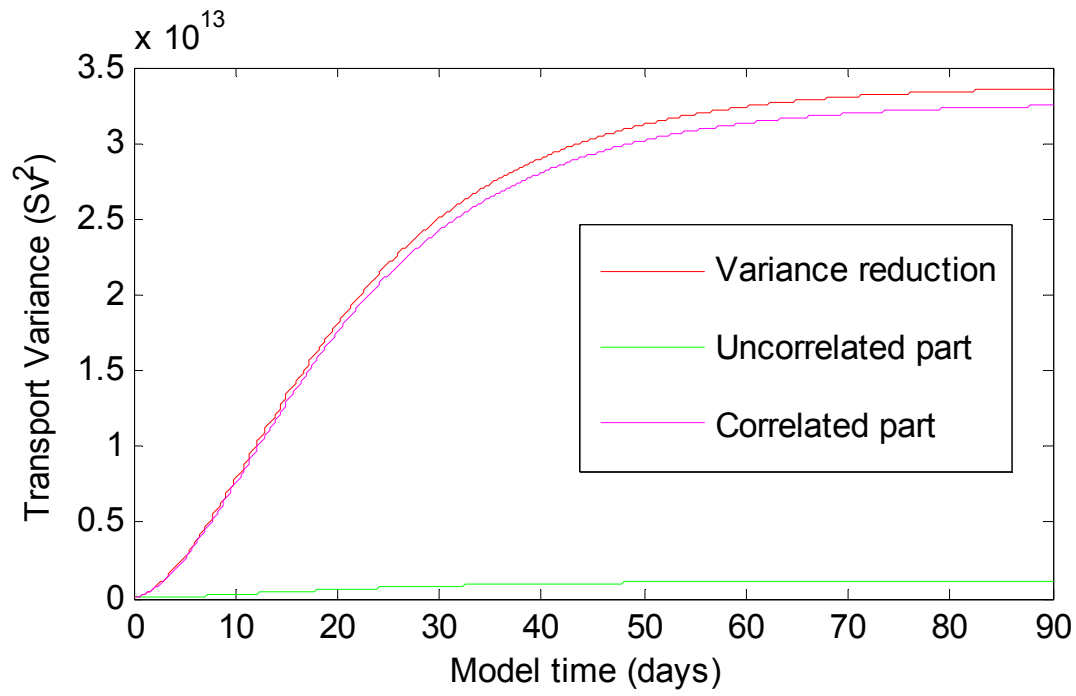

Figure 5.55 Same as Figure 5.53 but for Drake array altimetry assimilation 10 days after model initialization. 

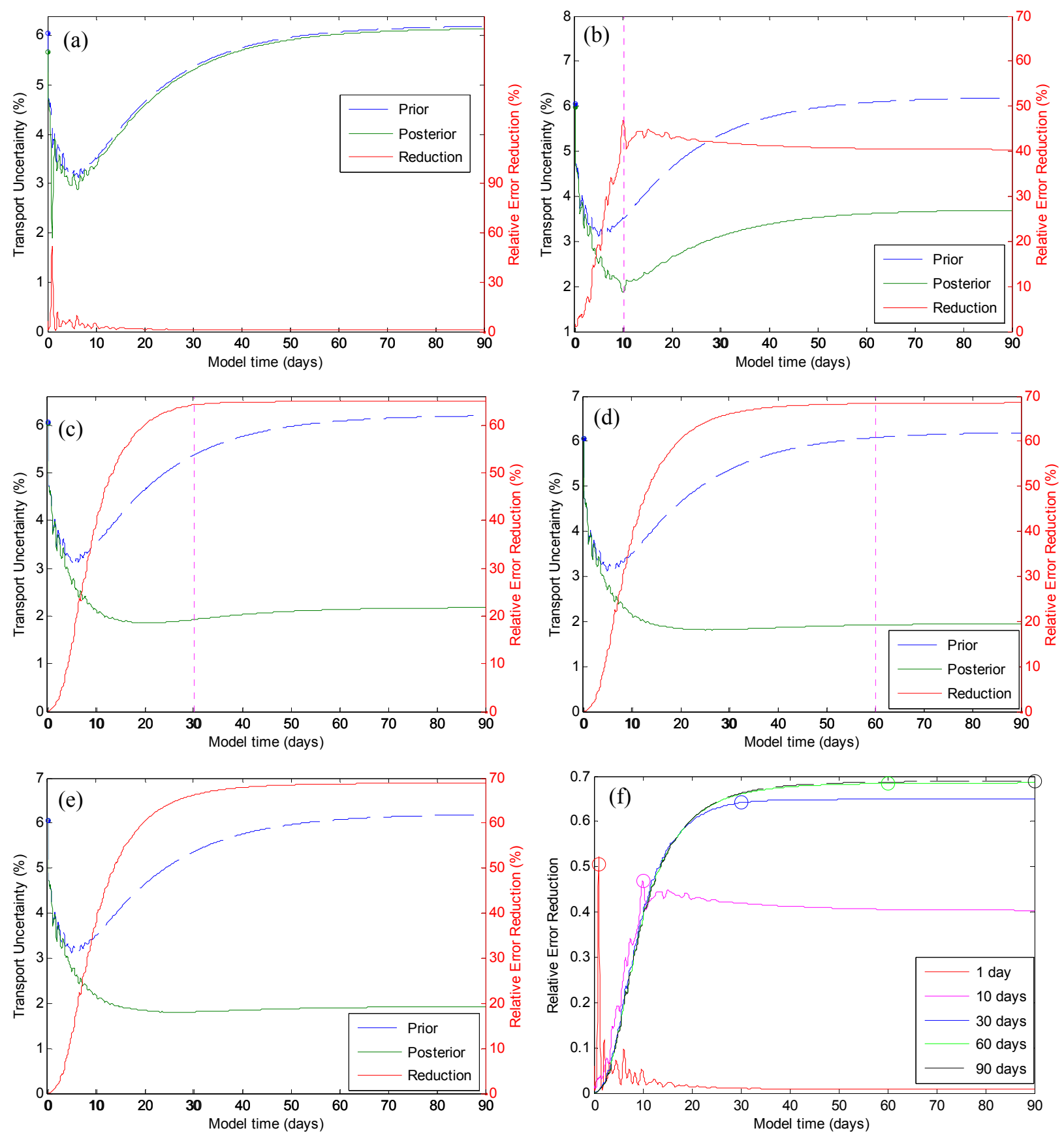

Figure 5.56 Same configuration as in Figure 5.50 but for different uncertainty assimilation times. Panel (a) shows the same data as Figure 5.50 but in single plot and for longer times. The left-side axis is transport uncertainty in percents, the right-side axis the uncertainty reduction in percents. Panels (b), (c), (d) and (e) show the prior and posterior uncertainties for 10,30,60 and 90 days uncertainty assimilation experiments. Panel (f) shows all 5 uncertainty reduction curves together in absolute units and with the different uncertainty assimilation times shown with open circles of corresponding colors.

Now we return to the comparison of different uncertainty assimilation experiments. Figure 5.56 displays the posterior uncertainty evolution results for the different 
assimilation times as listed in Table 5.2. The review of the detailed evolution allows to reexamine the above raised question - if the increase in uncertainty reduction with uncertainty assimilation period is "due to longer assimilation times or because the system integrates to a steady state equilibrium". Considering the 10 days uncertainty assimilation experiment we see that the peak $46.8 \%$ uncertainty reduction applies only to the exact assimilation moment, it recedes slightly to value of $40 \%$ at the steady state. As a result, the posterior uncertainty of the transport grows from $1.7 \%$ minimum to $3.7 \%$ at the steady state. For longer uncertainty assimilation periods the picture is quite different. The uncertainty reduction peak does not appear and it is monotonically increasing to the steady state values asymptoting to $70 \%$. The results for 30,60 and 90 days are very similar indicating the convergence of the uncertainty dynamics to steady sate equilibrium. All five experiments are compared to each other in a single plot in Figure 5.56(f). We see that the peak uncertainty reduction for 1 day assimilation experiment is larger than the reduction for 10 days experiment for all times. For the long assimilation period experiments the uncertainty reduction grows more slowly at first days, but reaches higher values as the system approaches the steady state.
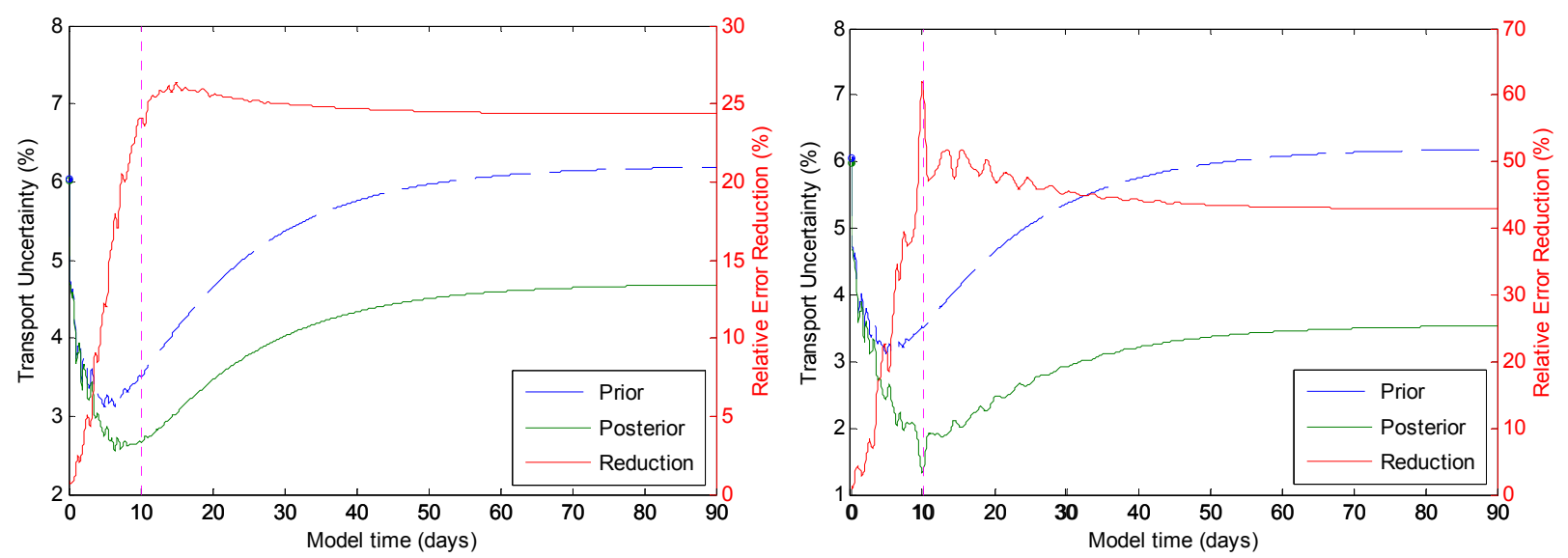

Figure 5.57 Same panels as Figure 5.56(b) but for 10 days assimilation of Drake transect altimetry (left plot) vs. Drake transect altimetry and currents (right plot).

We can also examine the uncertainty evolution for the other experiments listed in Table 5.2. Figure 5.57 compares assimilation of Drake transect altimetry vs. Drake transect altimetry and currents uncertainty. Both generally resemble the 10 days Drake array altimetry experiment (Figure 5.56b). The transect altimetry only case shows weaker uncertainty reduction than altimetry array case, and no transient peak at 10 days time is 
noticeable. While the table shows weakening of uncertainty reduction from $46.8 \%$ to $24.1 \%$ on day 10 , the figures show steady state reduction weakening of $40 \%$ to $24.3 \%$. The uncertainty constraint effects of assimilating altimetry with currents are stronger than both Drake altimetry transect and altimetry array. A strong uncertainty reduction peak is calculated on day 10 and transient wiggling of the posterior uncertainty curve is more pronounced.

The uncertainty evolution results for other prior selections are not qualitatively different. Here we only plot the relative uncertainty reduction rates for the three 90 days assimilation experiments with different wind stress priors (Table 5.3). Figure 5.58 shows that for the largest priors the relative uncertainty reduction is slightly larger, but for the weaker cases it does not make a noticeable difference.

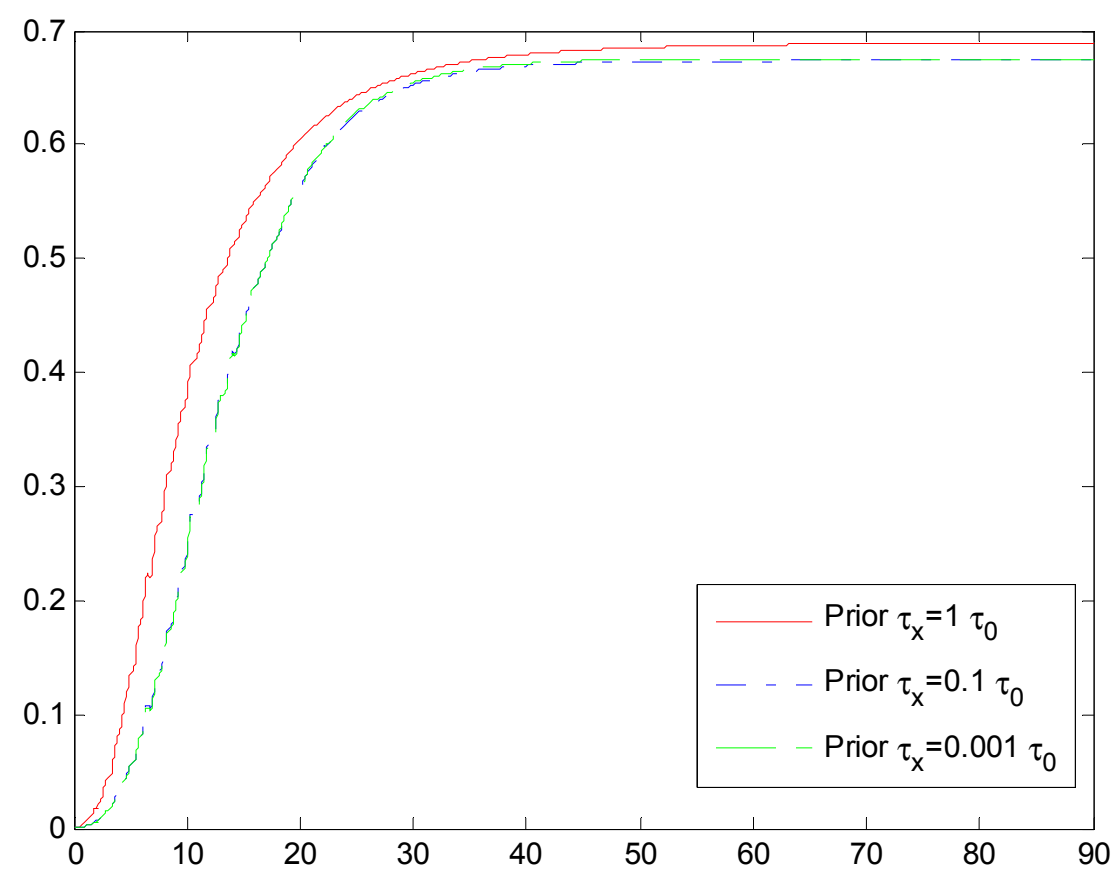

Figure 5.58 Same as Figure 5.56(f) for the three wind stress priors in Table 5.3. 


\subsubsection{Realistic bathymetry}

Next, we analyze the results of the forward uncertainty propagation for the realistic bathymetry configuration, summarized in Tables 5.5, 5.6 and 5.7. The leading eigenvalues are smaller, compared to the flat bottom configuration, for all the experiments except for the day 1 Drake array altimetry experiment. However, this higher than usual first eigenvector does not reflect the whole Hessian spectrum for this case as was seen in Figure 5.23. The convergence of uncertainty assimilation to the steady state is faster, as the eigenvalues for 30,60 and 90 days are indistinguishable.

\subsubsection{Prior uncertainty propagation}

The evolution of the prior uncertainty of the Drake Passage transport is very different from the flat bottom configuration and also between the different prior cases. For the prior control values in Table 5.5 the prior of the transport grows rapidly from $3.7 \%$ in the first 5 days (Figure 5.59) and converges to $9.2 \%$ in the steady state. This is the opposite of the initial prior drop and the eventual growth for the flat bottom cases, and the sharp drop with negligible growth for the smaller wind stress control priors in Table 5.7. Because the posterior uncertainties depend on dynamics of prior uncertainties, the evolutions of the posterior will also be very different between the flat and the realistic bathymetry configurations.

\subsubsection{Posterior uncertainty and uncertainty reduction}

The posterior uncertainties and the relative uncertainty reductions are affected by the different dynamics of the realistic bathymetry configuration. From Table 5.5 we learn that the peak uncertainty reductions at the uncertainty assimilation times are larger compared to the flat bottom configuration for all experiments except for the 10 days south Pacific altimetry. For the first day Drake array assimilation experiment it is only $55.9 \%$ vs. $50.6 \%$, but for the steady state Drake array assimilation it is $86.4 \%$ vs. $68.9 \%$. The largest instantaneous uncertainty reduction in Table 5.5 is almost $90 \%$ for 10 days Drake transect altimetry and current data, which is in dimensional units reduction of prior $9.2 \mathrm{~Sv}$ to $1 \mathrm{~Sv}$ only. 
Table 5.5 Summary of forward uncertainty propagation experiments for realistic bathymetry configuration with prior uncertainty of controls specified with a diagonal covariance matrix with constant standard errors: $\left[\Delta \tau^{\mathrm{x}}, \Delta \tau^{\mathrm{y}}, \Delta r, \Delta u_{t=0,} \Delta v_{t=0}, \Delta \eta_{t=0}\right]^{T}=[1 \mathrm{~Pa}, 1 \mathrm{~Pa}, 5.0 \mathrm{e}-3 \mathrm{~m} / \mathrm{s}, 0.01 \mathrm{~m} / \mathrm{s}, 0.01 \mathrm{~m} / \mathrm{s}, 0.1 \mathrm{~m}]^{T}$

\begin{tabular}{|lcccc|}
\hline & $\begin{array}{c}\text { Eigenvalue } \\
1\end{array}$ & $\begin{array}{c}\text { Prior } \\
\text { Uncertainty } \\
(\mathrm{Sv})\end{array}$ & $\begin{array}{c}\text { Posterior } \\
\text { Uncertainty } \\
(\mathrm{Sv})\end{array}$ & $\begin{array}{c}\text { Uncertainty } \\
\text { Reduction } \\
(\%)\end{array}$ \\
\hline 1 day Drake Array Altimetry & $1.84 \mathrm{E}+06$ & 5.123 & 2.261 & 55.9 \\
\hline 10days Drake Array Altimetry & $5.59 \mathrm{E}+05$ & 9.249 & 1.317 & 85.8 \\
\hline 30days Drake Array Altimetry & $5.98 \mathrm{E}+05$ & 9.650 & 1.311 & 86.4 \\
\hline 60days Drake Array Altimetry & $5.98 \mathrm{E}+05$ & 9.662 & 1.311 & 86.4 \\
\hline 90days Drake Array Altimetry & $5.98 \mathrm{E}+05$ & 9.662 & 1.311 & 86.4 \\
\hline 10days Drake Transect Altimetry & 1.78E+05 & 9.249 & 3.295 & 64.4 \\
\hline 10days South Pacific Transect Altimetry & $1.42 \mathrm{E}+05$ & 9.249 & 8.978 & 2.92 \\
\hline 10days North Pacific Transect Altimetry & $6.73 \mathrm{E}+03$ & 9.249 & 9.204 & 0.489 \\
\hline 10days Drake Transect Altimetry \& Flow & $3.78 \mathrm{E}+06$ & 9.249 & 0.991 & 89.3 \\
\hline
\end{tabular}

Table 5.6 Same as Table 5.5 except for different prior uncertainties of wind stress as specified.

\begin{tabular}{|lcccc|}
\hline & $\begin{array}{c}\text { Eigenvalue } \\
1\end{array}$ & $\begin{array}{c}\text { Prior } \\
\text { Uncertainty } \\
(\mathrm{Sv})\end{array}$ & $\begin{array}{c}\text { Posterior } \\
\text { Uncertainty } \\
(\mathrm{Sv})\end{array}$ & $\begin{array}{c}\text { Uncertainty } \\
\text { Reduction } \\
(\%)\end{array}$ \\
\hline $\begin{array}{l}\text { 90days Drake Array Altimetry, } \\
\text { Prior } \Delta \tau=1 \mathrm{~Pa}\end{array}$ & $5.98 \mathrm{E}+05$ & 9.662 & 1.311 & 86.4 \\
\hline $\begin{array}{l}90 \text { days Drake Array Altimetry, } \\
\text { Prior } \Delta \tau=0.1 \mathrm{~Pa}\end{array}$ & $5.98 \mathrm{E}+05$ & 1.666 & 0.704 & 57.8 \\
\hline $\begin{array}{l}90 \text { days Drake Array Altimetry, } \\
\text { Prior } \Delta \tau=0.0001 \mathrm{~Pa}\end{array}$ & $5.98 \mathrm{E}+05$ & 1.364 & 0.642 & 53.0 \\
\hline
\end{tabular}

Table 5.7 Same as Table 5.5 for realistic bathymetry configuration for prior uncertainty of controls with standard errors: $\left[\Delta \tau^{\mathrm{x}}, \Delta \tau^{\mathrm{y}}, \Delta r, \Delta u_{t=0,} \Delta v_{t=0}, \Delta \eta_{t=0}\right]^{T}=[1.0 \mathrm{e}-4 \mathrm{~Pa}, 1.0 \mathrm{e}-4 \mathrm{~Pa}, 5.0 \mathrm{e}-3 \mathrm{~m} / \mathrm{s}, 0.01 \mathrm{~m} / \mathrm{s}, 0.01 \mathrm{~m} / \mathrm{s}, 0.1 \mathrm{~m}]^{T}$.

\begin{tabular}{|lcccc|}
\hline & $\begin{array}{c}\text { Eigenvalue } \\
1\end{array}$ & $\begin{array}{c}\text { Prior } \\
\text { Uncertainty } \\
(\mathrm{Sv})\end{array}$ & $\begin{array}{c}\text { Posterior } \\
\text { Uncertainty } \\
(\mathrm{Sv})\end{array}$ & $\begin{array}{c}\text { Uncertainty } \\
\text { Reduction } \\
(\%)\end{array}$ \\
\hline 1 day Drake Array Altimetry & $1.84 \mathrm{E}+06$ & 2.931 & 1.933 & 34.1 \\
\hline 10days Drake Array Altimetry & $5.59 \mathrm{E}+05$ & 1.388 & 0.690 & 50.3 \\
\hline 30days Drake Array Altimetry & $5.98 \mathrm{E}+05$ & 1.362 & 0.641 & 52.9 \\
\hline 60days Drake Array Altimetry & $5.98 \mathrm{E}+05$ & 1.364 & 0.642 & 53.0 \\
\hline 90days Drake Array Altimetry & $5.98 \mathrm{E}+05$ & 1.364 & 0.642 & 53.0 \\
\hline 10days Drake Transect Altimetry & $1.78 \mathrm{E}+05$ & 1.388 & 1.212 & 12.6 \\
\hline 10days South Pacific Transect Altimetry & $1.42 \mathrm{E}+05$ & 1.388 & 1.293 & 6.87 \\
\hline 10days North Pacific Transect Altimetry & $6.73 \mathrm{E}+03$ & 1.388 & 1.362 & 1.85 \\
\hline 10days Drake Transect Altimetry \& Flow & $3.78 \mathrm{E}+06$ & 1.388 & 0.789 & 43.1 \\
\hline
\end{tabular}




\subsubsection{Transport uncertainty constraint by different prior uncertainties of wind forcing}

Examining the dependence on the prior uncertainty of the controls (Table 5.6) shows much stronger dependence than in the flat bottom case. The more certain wind stresses lead to significantly lesser reduction of uncertainty of the transport in the steady state. But this may be simply understood noticing much lower prior uncertainty of the transport in the steady state. It is already so low that more observations do not reduce it as much. The lower prior uncertainty of the winds changes the uncertainty results for all the experiments in Table 5.7. The differences between the experiments are less than in Table 5.5 , with maximum reductions not reaching $90 \%$ but only $50 \%$. Note, that the relative reductions for both Pacific transects are larger, $6.9 \%$ and $1.9 \%$, compared to the weakly a priori constrained case.
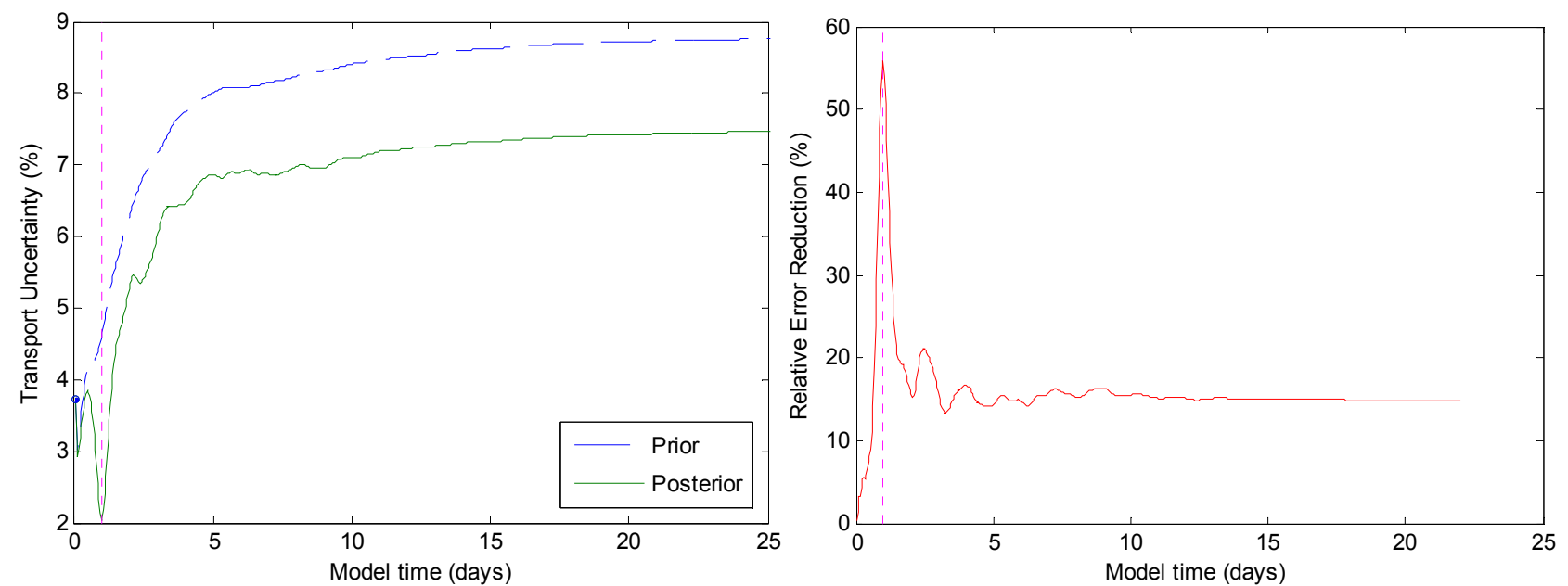

Figure 5.59 Evolution of prior and posterior uncertainties of Drake Passage transport given in percents as fraction of the actual transport at the presented time. Here shown for 1 day Drake array altimetry uncertainty assimilation experiment for realistic bathymetry configuration model. The prior uncertainties are given by the standard errors: $\left[\Delta \tau^{\mathrm{x}}, \Delta \tau^{\mathrm{y}}, \Delta r, \Delta u_{t=0}, \Delta v_{t=0}, \Delta \eta_{t=0}\right]^{T}=[1 \mathrm{~Pa}, 1 \mathrm{~Pa}, 5.0 \mathrm{e}-3 \mathrm{~m} / \mathrm{s}, 0.01 \mathrm{~m} / \mathrm{s}$, $0.01 \mathrm{~m} / \mathrm{s}, 0.1 \mathrm{~m}]^{T}$. The left panel compares the prior uncertainty (blue dashed) and the posterior uncertainty (green solid) evolutions. The moment of uncertainty assimilation, here 1 day, is shown by the vertical magenta dotted line. The right panel shows the relative uncertainty reduction fraction in percents (red solid) and a the time of uncertainty assimilation (vertical magenta dotted). 

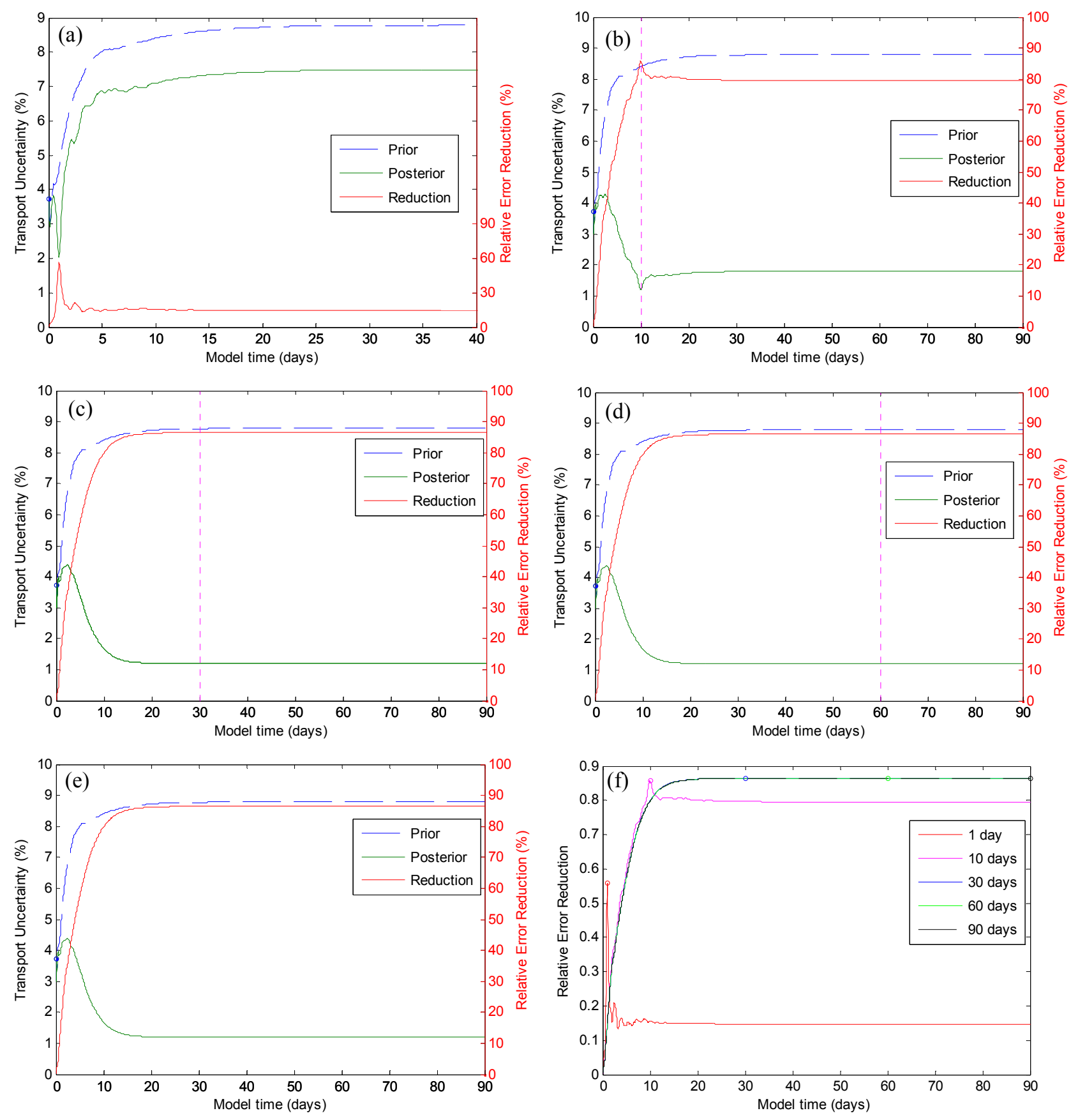

Figure 5.60 Same configuration as in Figure 5.59 but for different uncertainty assimilation times. Panel (a) shows the same data as Figure 5.59 but in single plot and for longer times. The left-side axis is transport uncertainty in percents, the right-side axis the uncertainty reduction in percents. Panels (b), (c), (d) and (e) show the prior and posterior uncertainties for 10,30, 60 and 90 days uncertainty assimilation experiments. Panel (f) shows all 5 uncertainty reduction curves together in absolute units and with the different uncertainty assimilation times shown with open circles markers of corresponding colors. 

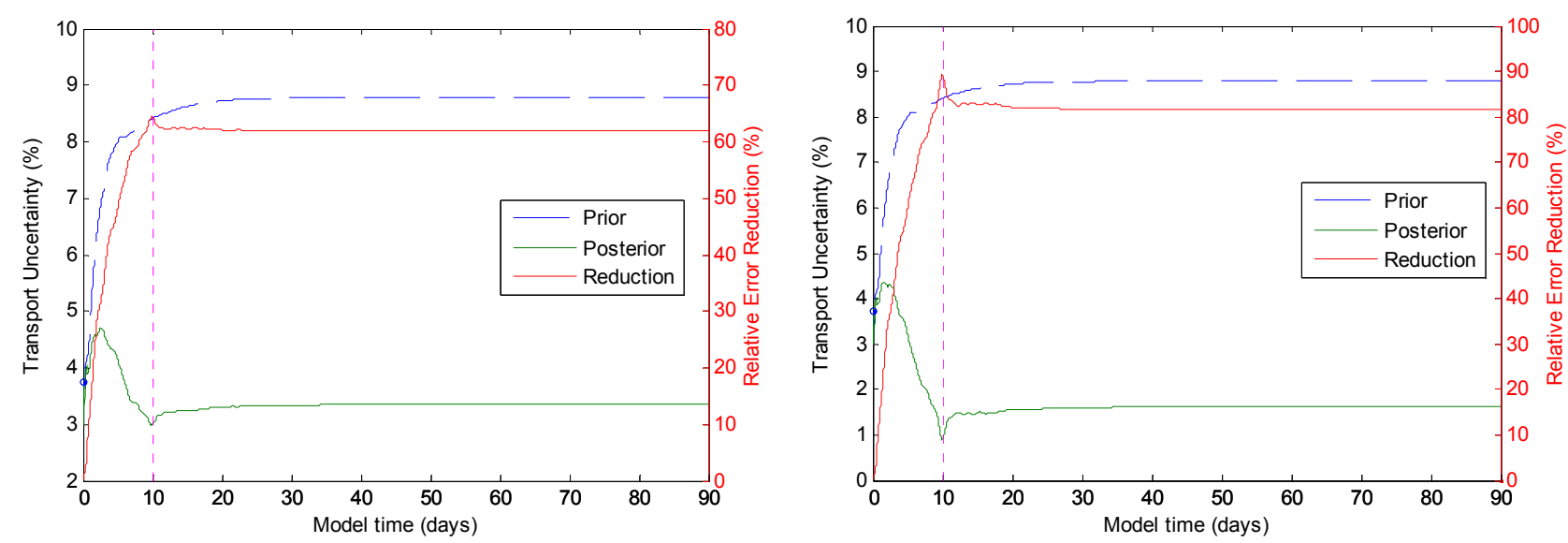

Figure 5.61 Same panels as Figure 5.60(b) but for 10 days assimilation of Drake transect altimetry (left plot) vs. Drake transect altimetry and currents (right plot).
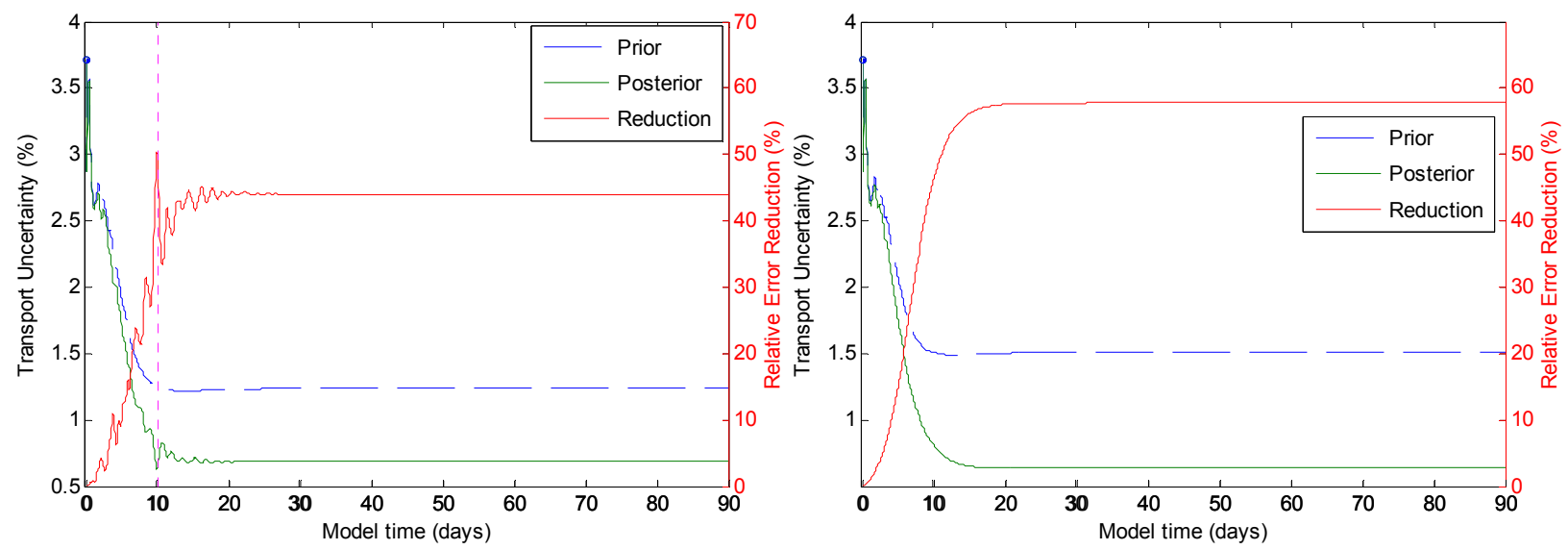

Figure 5.62 Same panels as Figure 5.60(b) but for assimilation of Drake array altimetry on day 10 (left plot) vs. on day 90 (right plot) with weaker prior uncertainties of wind stress as given in Table 5.7.

\subsubsection{Time-resolving analysis of uncertainty dynamics}

Finally, we examine the evolution of the posterior uncertainties of the transport for the realistic bathymetry configuration. As mentioned above, the major difference from the flat bottom cases is the evolution of the prior uncertainty, which is monotonously growing for the priors in Table 5.5. Figure 5.59 shows in detail the uncertainties for the first day Drake array altimetry assimilation. The sharp uncertainty reduction peak in the first day more than halves the prior transport uncertainty, but eventually the posterior uncertainty grows with the prior. The steady state uncertainty reduction is $15 \%$. This and the other Drake array altimetry cases are compared further in Figure 5.60. Uncertainty reduction for the 10 day case resembles that for the flat bottom, with rapid growth, a peak and slight decline to the steady state. Uncertainty reduction for the longer assimilation 
periods also resembles the flat bottom results, except that the initial growth is more rapid without the convex bend of the curve. All the 5 cases are plotted together on Figure 5.60(f), showing the convergence of the 30 and 60 days experiments to the steady state curve. The most essential result is that the assimilation of the observations uncertainty significantly reduces the prior uncertainties of transport, in fact reversing the dynamical tendency of the uncertainty to grow with time.

Further, we compare the assimilation of Drake transect altimetry with altimetry together with currents data in Figure 5.61. Again, as in the flat bottom cases, the currents increase the uncertainty reduction effect but not by as much. The peak on the 10th day is again more prominent with the currents data. The wiggling of the posterior uncertainty curve for that case is not as vigorous.

Next we examine evolution of the uncertainties calculated for different selected priors. Figure 5.62 illustrates the evolution of 10 and 90 day Drake array experiments from Table 5.7. The prior uncertainty of Drake transport is different, rapidly dropping and staying low because the a priory well constrained winds reduce the prior uncertainty of the transport as soon as the model settles in. The relative uncertainty reduction evolution curves are also different. For the 10 days experiment a strong peak appears and after it relaxes the reduction continues to grow to a steady state with considerable amount of wiggling. For the 90 days experiment the transient growth of reduction slows down temporarily as the convex bend appears. The evolutions of uncertainty reduction curves for the experiments in Table 5.6 are shown in Figure 5.63. Unlike in the flat bottom cases (Figure 5.58) the prior uncertainty of the winds affects a lot the posterior uncertainty reduction. 


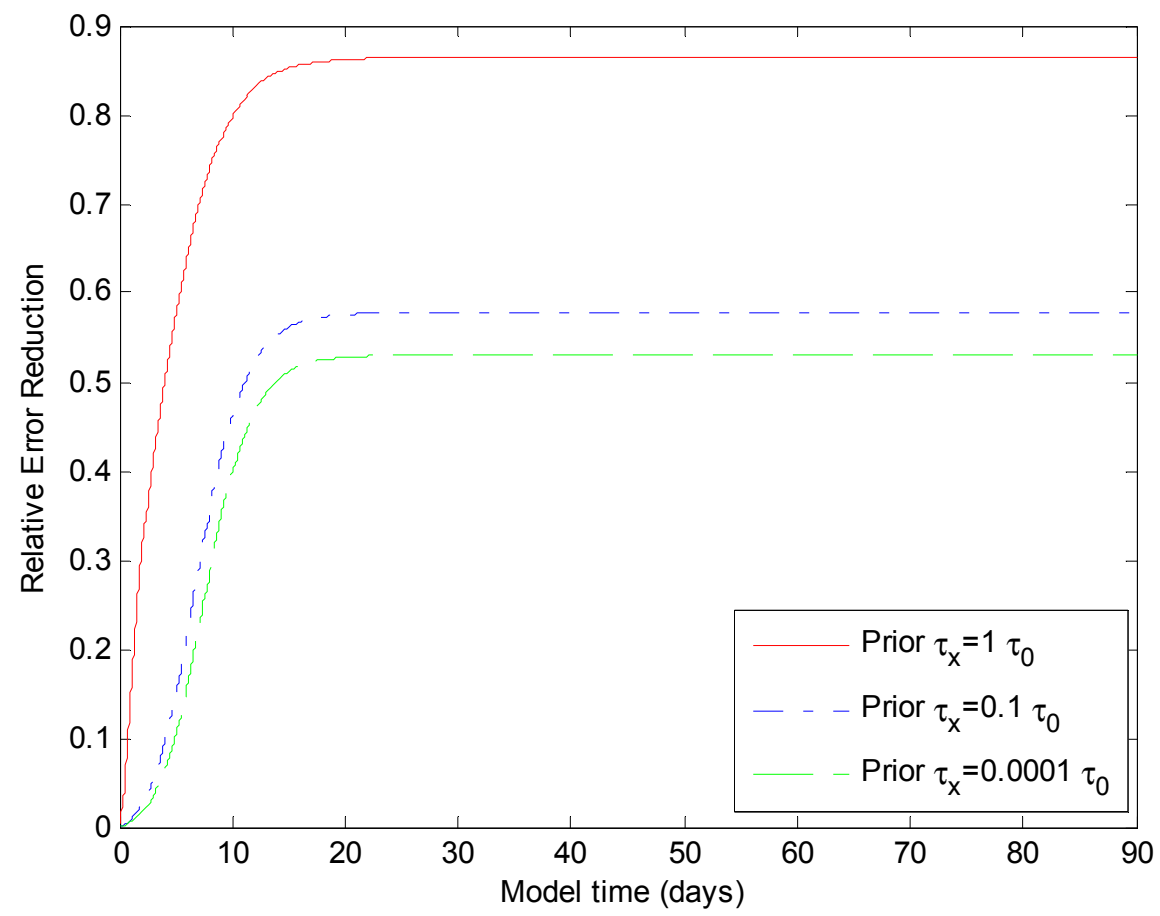

Figure 5.63 Same as Figure 5.60(f) for the three wind stress priors in Table 5.36. 


\section{Summary and Conclusions}

The central achievements of this dissertation are the development and implementation of a formal uncertainty quantification (UQ) methodology for large scale ocean state estimation. A methodology is developed in an inverse-forward ocean modeling framework (Section 2.1) tailored to extend the Lagrange multipliers state estimation machinery used in ECCO. Observation-constrained ocean state and model controls are estimated with the inverse model. The forward model calculates the oceanographic target quantities, such as the circumpolar transport, which are not among the variables estimated by the inverse model.

UQ methodology is based on application of the Hessian and the Jacobian derivative codes of a nonlinear ocean model. The derivatives retain the nonlinearity of the ocean dynamics, but provide a linearized leading order description of uncertainty propagation. Although other methodologies exist for a higher order uncertainty description, their applicability on the scale of realistic ocean state estimation problems is limited by the large dimension. A scalable UQ algorithm is developed, applicable in the dynamically consistent state estimation framework of the adjoint method. Unlike sequential reanalysis approaches, it is free of physical imbalances due to data assimilation cycles and does not require propagating large covariance matrices throughout the model.

Inverse and forward propagation of uncertainty is quantified by the developed UQ scheme. Inverse propagation was the main challenge and was solved by inversion of the Hessian matrix of model-observations misfit. The curvature of the misfit function described by the Hessian eigenvalues is a measure of confidence in the value of the inverse problem solution - larger curvature meaning better fit. Uncertainty of the solution is given by the inverse of these eigenvalues, paired with eigenvectors corresponding to the orthogonal combinations of the constrained control fields. The inverted Hessian matrix is associated with the covariance of the solution vector. Its pseudo-inverse is the solution covariance in a reduced data-supported solution subspace.

Justification of the Hessian approach was demonstrated by direct derivation of inverse uncertainty propagation for linear invertible models, by derivation of the linearized 
solution of nonlinear inverse problem, and by connection to the Gauss-Markov, Bayesian and maximum likelihood state estimation approaches. Main technical challenges of this thesis were calculation and inversion of the Hessian matrix by differentiation of a numerical ocean model over a large high-dimensional variable space. A Hessian ocean model was constructed by direct second order differentiation with algorithmic differentiation (AD) machinery and implemented as a Hessian-by-vector product operator. This analytically differentiated Hessian code differs from linearized Hessian models used in variational data assimilation and does not neglect the nonlinear part in large residual configurations. Reduced rank representation of the Hessian matrix resolves the large dimensionality limitations, here $O\left(10^{5}\right)$, discarding the unobservable nullspace of the estimation problem. In practical applications where only a limited number of degrees of freedom is constrained by observations, this results in a lossless compression of the Hessian matrix inversion. A Lanczos algorithm is applied for spectral decomposition of the Hessian and for partition of the control parameter space to the dataconstrained range and the unobservable nullspace. Numerical algorithms are designed with matrix-free linear algebra, allowing the computational scalability required for the large dimensionality of the realistic ocean state estimation problem.

Connection of formal uncertainty propagation to sensitivity analysis is clarified by the structure of the UQ methodology constructs. Model sensitivity fields, given by the first derivative of ocean model, resolve the mathematical links between perturbations of model inputs and outputs, and therefore are used to describe the effects of uncertainty in one on the other. Formal uncertainty algebra combines these sensitivity links in quadratic structures projecting the uncertainty between model input and output domains. Forward projection for independent uncertainties is given by the quadrature rule, summing squares of sensitivities scaled by input uncertainties. For correlated input uncertainties, the quadrature rule is extended to include a double summation of sensitivity cross-products with corresponding covariances. For inverse uncertainty projection, inversion of the Hessian is the inversion of weighted outer products of sensitivities. The number of linearly independent sensitivity patterns defines the independent degrees of freedom and is the basis for compression of the algorithm. Moreover, the role of sensitivity in 
uncertainty propagation leads to identification of the adjoint dynamics as the physical mechanisms of uncertainty, as demonstrated by the adjoint barotropic waves.

The developed UQ methodology is applied to the barotropic uncertainty of the Antarctic Circumpolar Current transport through the Drake Passage. We applied a hierarchy of analytical and numerical models of increasing complexity to resolve the barotropic mechanisms of the ACC and to guide the UQ methodology calibration. An analytical ACC model allowed a detailed understanding of the structure of the inverse and forward uncertainty propagation, the central role of sensitivities in the algorithm, the singularity of the Hessian and the structure of its pseudo-inverse. The analytical form of UQ is explicitly time-resolving, highlighting its multiple time dependencies, revealing the stationary and transient uncertainty regimes, explaining the differences between initial and boundary conditions uncertainty dynamics and their cross-coupling. Numerical models of the ACC with barotropic MITgcm configuration resolve the dynamics of uncertainty at the steady state equilibrium of wind forcing and bottom friction. Comparison of flat and realistic bottom topography configurations allows distinction between closed and blocked geostrophic contour governed ACC dynamics

An identical twin setup eliminates any residual misfit of the state estimation solution. Synthetic observations are generated with forward MITgem runs modeling the typical observation systems deployed at the Drake Passage. Different uncertainty assimilation experiments simulate several generic ACC monitoring configurations, comparing the effects of different locations and geographic coverage, number of sensors deployed, measured variable, and different uncertainty assimilation times. Different control priors are tested and compared. Idealized model configurations guide identification of the barotropic uncertainty mechanisms by reducing the geometric complexity and simplifying the dynamical balances. Adjoint barotropic sensitivity waves are identified and lead to detection of barotropic uncertainty waves. Initial conditions sensitivities exhibit transient oscillations and decay to zero at the steady state. Boundary conditions sensitivities grow from zero to their stationary patterns. The evolution of sensitivities in the numerical model is consistent with the exponential single timescale sensitivity dynamics predicted by the idealized analytical model. Thus, the leading order dynamics of both sensitivity and uncertainty is explained by the frictional time scale of relaxation to 
wind forcing-bottom drag equilibrium. A key conclusion is that even when the model is initialized with the steady state initial conditions and remains in equilibrium at all times, if finite uncertainty is assumed about its initial and boundary conditions, the uncertainty simulations are not time invariant and adjust to the steady state with the forward model relaxation timescale.

The developed UQ scheme includes separate calculations for prior-independent uncertainty assimilation and for uncertainty assimilation with prior information leading to posterior uncertainty reduction. The former is an unbiased pure inverse uncertainty propagation algorithm, inverting the unregularized Hessian of model-data misfit and resolving the constrained information patterns in Hessian eigenvectors. The latter depends on sometimes arbitrary prior selection, and its assimilated information patterns may be masked by the patterns of the prior unless they are orthogonal complements of each other. Prior-to-posterior uncertainty reduction in the control space constrains the diagonal terms of the posterior covariance, which are visualized by plotting the relative uncertainty reduction maps. In addition to the diagonal terms decrease, the uncertainty assimilation procedure introduces non-zero off-diagonal terms in the covariance. The offdiagonal terms resolve the correlations between model controls, revealing the physical mechanisms of dynamical coupling within and between the different control fields. Together, the constrained diagonal and off-diagonal posterior control uncertainty terms constrain the posterior error of the circumpolar transport in forward uncertainty propagation calculations. Comparing the effects of the diagonal and the off-diagonal terms we find that transport uncertainty reduction due to the observation-constrained variances of controls is relatively small. Most of the reduction in transport uncertainty is shown to be due to the off-diagonal terms, highlighting the importance of correlations.

Future research directions include extending the formal quantitative framework of the current uncertainty bounds study to the field of statistical inference to formally quantify confidence regions, goodness of fit and uncertainty partition between resolved and unresolved uncertainty sources. The next technical challenge is to integrate the developed machinery in the full realistic configuration of the ECCO ocean state estimation system. This will require extension of the machinery from synthetic to real observed data and to include baroclinic ocean physics. The developed UQ method is can be applied for model 
calibration to guide selection of physical parameterizations, boundary conditions, numerical parameters. The uncertainty assimilation methodology is applicable to new observation systems design by quantifying the expected information gains and optimizing for the specific observation goals. 


\section{Appendix}

\section{A. Maximum likelihood and Bayesian formulation of the inverse problem}

Consider the model-data misfit cost function (2.2.1) for a linear forward model M. We note that the quadratic form of the cost function resembles closely the negative of the argument of the exponent in multivariate Gaussian distribution, the minimum $\hat{\mathbf{x}}$ corresponding to the mode of the PDF

$$
N(\hat{\mathbf{x}}, \Sigma) \propto \exp \left[-(\mathbf{x}-\hat{\mathbf{x}})^{T} \Sigma^{-1}(\mathbf{x}-\hat{\mathbf{x}})\right]
$$

We therefore can quantify the likelihood of state $\mathbf{x}$ given the data $\mathbf{y}$ by the conditional probability of data $\mathbf{y}$ given the state $\mathbf{x}$

$$
L(\mathbf{x} \mid \mathbf{y}) \equiv P(\mathbf{y} \mid \mathbf{x}) \propto \exp \left[-J_{1}(\mathbf{y}, \mathbf{x})\right]
$$

if we assume that the observed data is normally distributed around model prediction with covariance $\mathbf{R}$, or equivalently, that the observation error $\mathbf{n}=\mathbf{y}-\mathbf{M x}$ is Gaussian with zero mean and covariance $\mathbf{R}$. Consequently, we can name the negative of the argument of the exponent ${ }^{13}$ (A.2), which is equal to the misfit function (2.2.1) - the likelihood cost function of state $\mathbf{x}$. The argument of the minimum of $J_{1}(\mathbf{x})$ is the most likely state $\mathbf{x}$ given the observations. Or equivalently, in the language of maximum likelihood estimation - the most likely state $\hat{\mathbf{x}}$ is one that maximizes the likelihood function (A.2) for given observations $\mathbf{y}$.

The probabilistic framework of the inverse problem can be extended by application of the Bayes' theorem to quantify the posterior uncertainly of the state $\mathbf{x}$ given observations $\mathbf{y}$

$$
P(\mathbf{x} \mid \mathbf{y})=P(\mathbf{y} \mid \mathbf{x}) \cdot P(\mathbf{x}) / P(\mathbf{y})
$$

If the prior probability of the state $\mathbf{x}$ is known and assumed Gaussian with mean $\mathbf{x}_{0}$ and covariance $\mathbf{P}_{0}$, allowing a definition of the negative logarithm of the prior probability as the prior cost function:

$$
J_{0}=\frac{1}{2}\left(\mathbf{x}-\mathbf{x}_{0}\right)^{T} \mathbf{P}_{0}^{-1}\left(\mathbf{x}-\mathbf{x}_{0}\right),
$$

\footnotetext{
${ }^{13}$ Also known as the negative log likelihood.
} 
then the posterior PDF of state $\mathbf{x}$ is given, up to multiplying factor independent of $\mathbf{x}$, by the exponent of the combined cost function

$$
J=J_{1}+J_{0}=\frac{1}{2}\left[(\mathbf{M x}-\mathbf{y})^{T} \mathbf{R}^{-1}(\mathbf{M x}-\mathbf{y})+\left(\mathbf{x}-\mathbf{x}_{0}\right)^{T} \mathbf{P}_{0}^{-1}\left(\mathbf{x}-\mathbf{x}_{0}\right)\right],
$$

which can be named the posterior cost function, or equivalently the negative log posterior.

\section{B. MITgcm configuration details}

Listed here for the realistic bathymetry simulations, see Section 3.3.4.

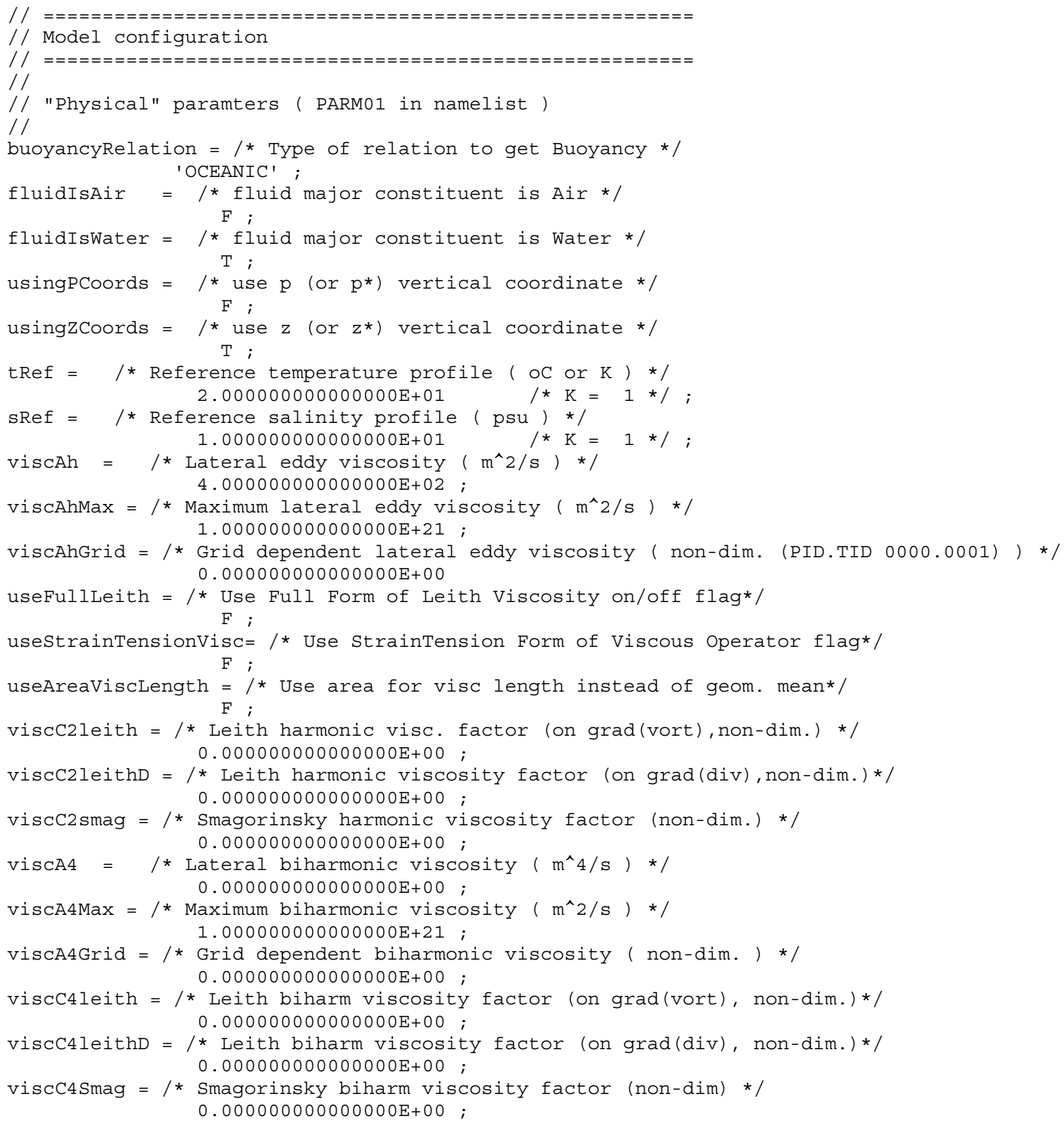




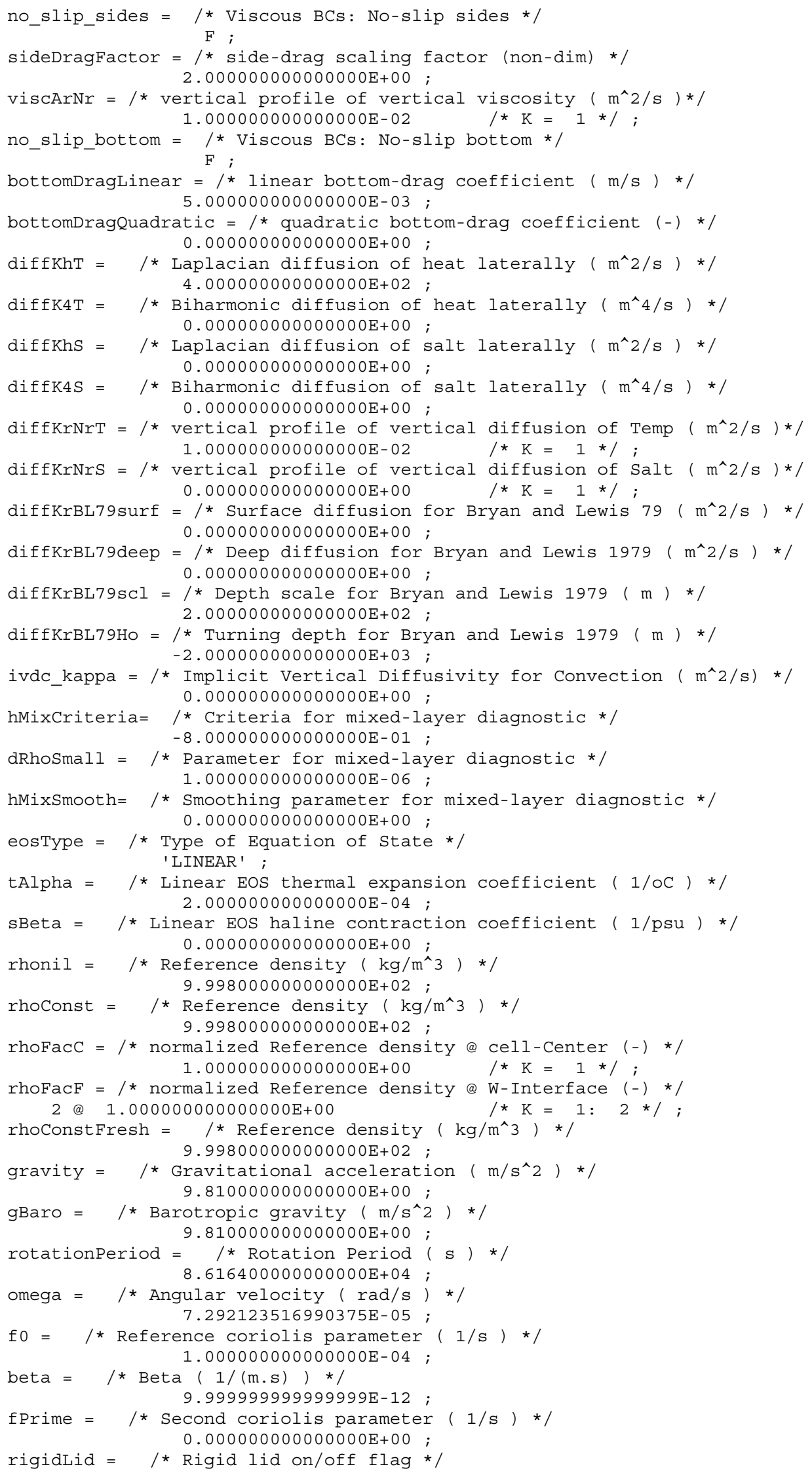




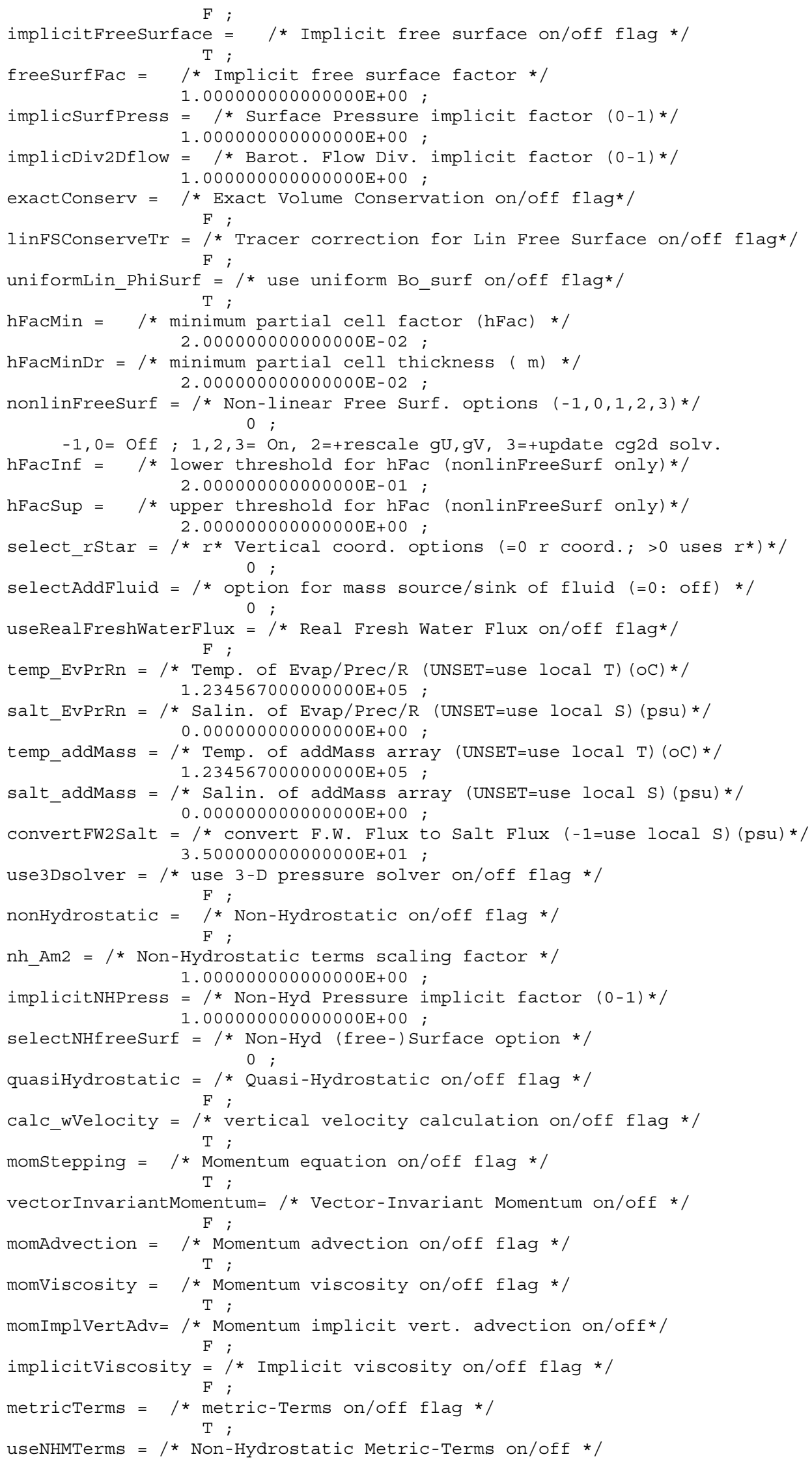




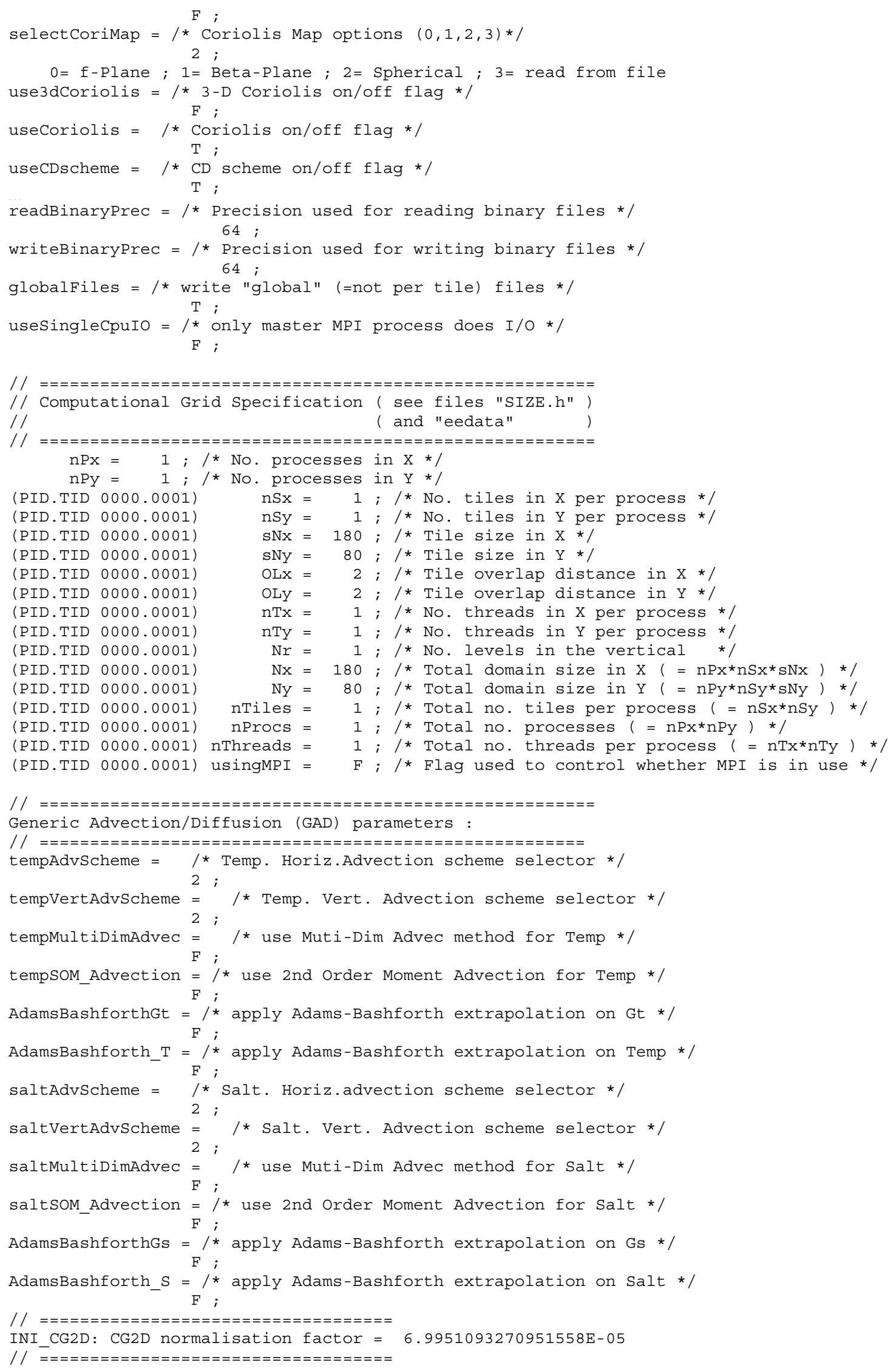




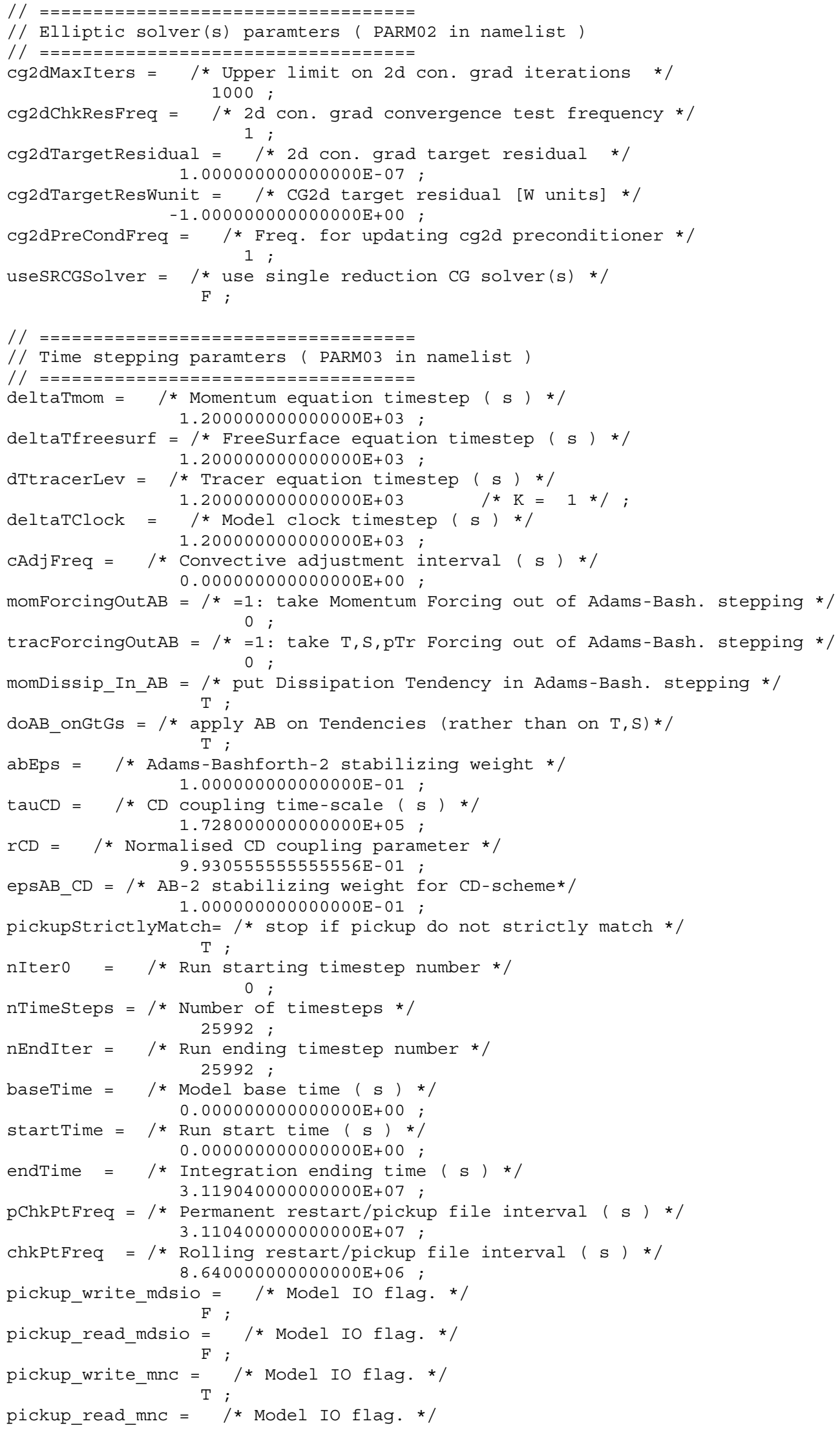




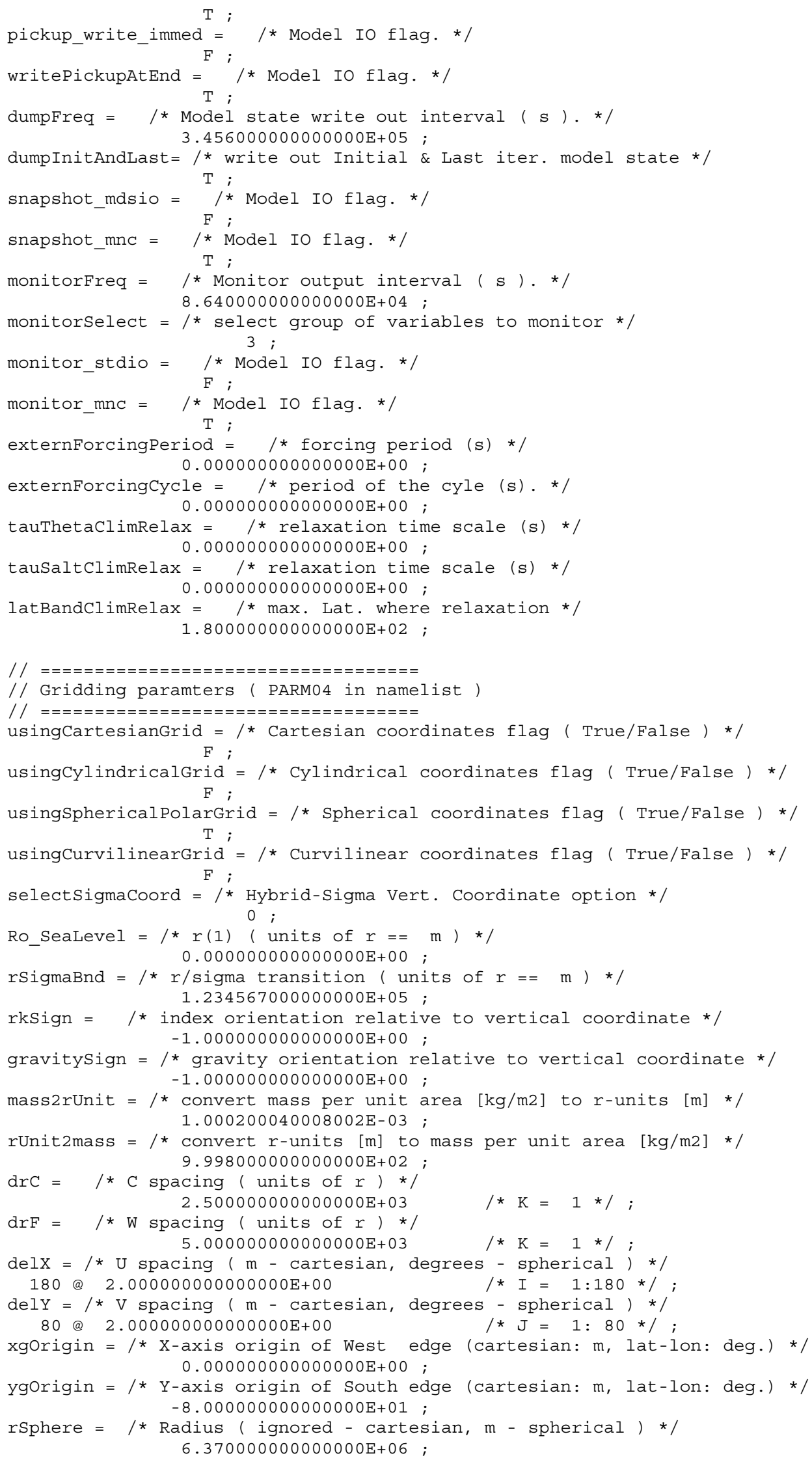




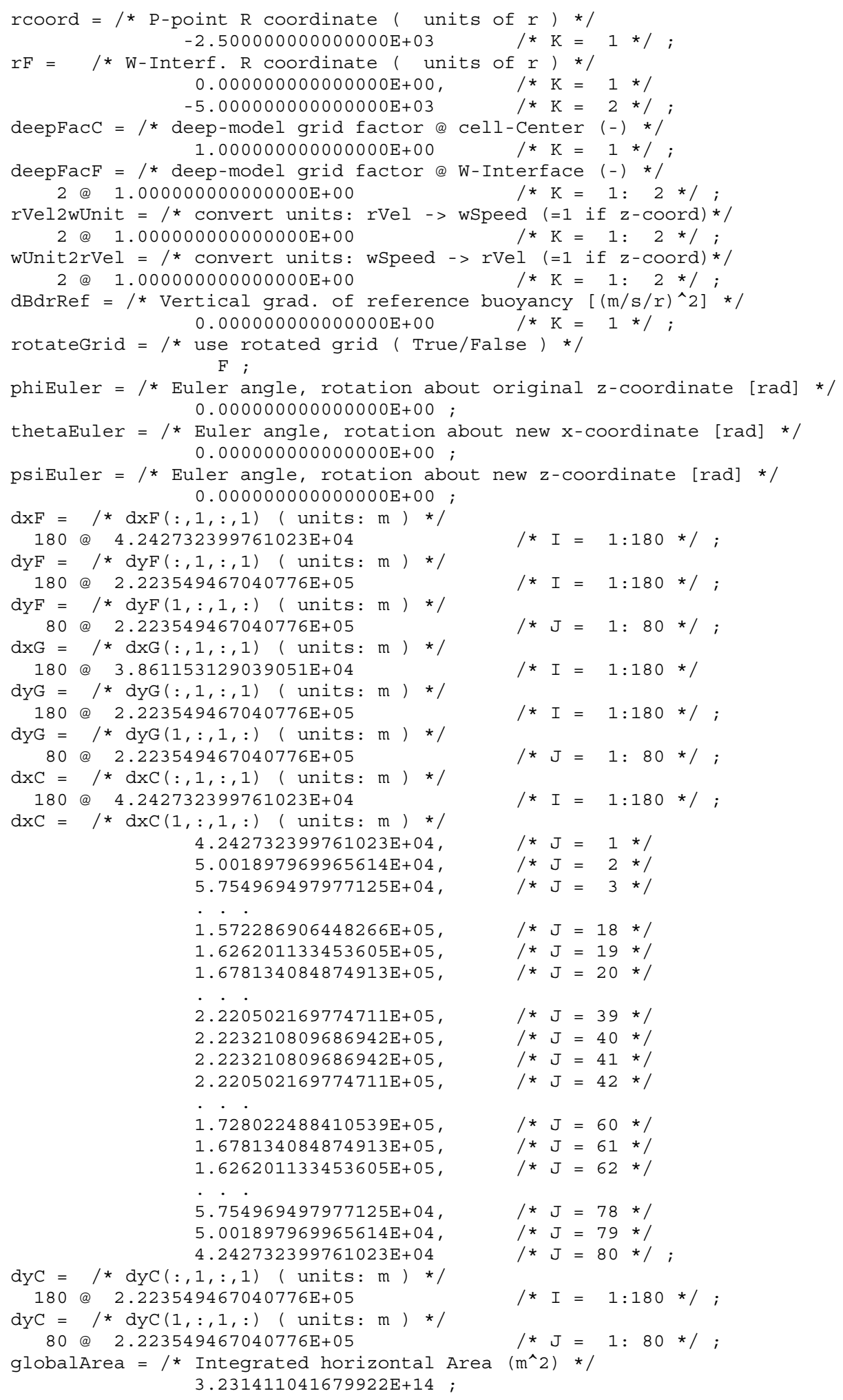




\section{References}

Adcroft, A., C. Hill, and J. Marshall, 1999: A new treatment of the Coriolis terms in Cgrid models at both high and low resolutions. Mon. Weather Rev., 127, 1928-1936.

Clowes, A., 1933: Influence of the Pacific on the circulation in the southwest Atlantic Ocean. Nature, 131, 189-191.

Cushman-Roisin, B., and J. M. Beckers, 2011: Introduction to geophysical fluid dynamics: physical and numerical aspects. Academic Press, Int. Geophys. Ser., 101.

Efron, B., and D. V. Hinkley, 1978: Assessing the accuracy of the maximum likelihood estimator: Observed versus expected Fisher information. Biometrika, 65, 457-483.

Forget, G., and C. Wunsch, 2007: Estimated global hydrographic variability. J. Phys. Oceanogr., 37, 1997-2008.

Giering, R., and T. Kaminski, 1998: Recipes for adjoint code construction. ACM Transactions on Mathematical Software (TOMS), 24, 437-474.

Giering, R., T. Kaminski, and T. Slawig, 2005: Generating efficient derivative code with TAF: adjoint and tangent linear Euler flow around an airfoil. Future Generation Comput. Syst., 21, 1345-1355.

Gilbert, J. C., and C. Lemaréchal, 1989: Some numerical experiments with variablestorage quasi-Newton algorithms. Math. Program., 45, 407-435.

Griewank, A. and A. Walther, 2008: Evaluating derivatives: principles and techniques of algorithmic differentiation. $429 \mathrm{pp}$, SIAM.

Gunson, J. R., and P. Malanotte-Rizzoli, 1996: Assimilation studies of open-ocean flows 2. Error measures with strongly nonlinear dynamics. Journal of Geophysical Research, 101, 28473-28,488.

Heimbach, P., C. Wunsch, R. M. Ponte, G. Forget, C. Hill, and J. Utke, 2011: Timescales and regions of the sensitivity of Atlantic meridional volume and heat transport: Toward observing system design. Deep Sea Research Part II: Topical Studies in Oceanography, 58, 1858-1879.

Kamenkovich, V., 1962: On the theory of the Antarctic circumpolar current (in Russian): Trudy Instituta Okeanologii, 56, 245-306.

Kaminski, T., M. Scholze, and S. Houweling, 2010: Quantifying the benefit of A - SCOPE data for reducing uncertainties in terrestrial carbon fluxes in CCDAS. Tellus $B, 62,784-796$.

Krupitsky, A., V. M. Kamenkovich, N. Naik, and M. A. Cane, 1996: A linear equivalent barotropic model of the Antarctic Circumpolar Current with realistic coastlines and bottom topography. J. Phys. Oceanogr., 26, 1803-1824.

LaCasce, J., P. Isachsen, 2010: The linear models of the ACC. Prog. Oceanogr., 84, 139157.

Lea, D. J., M. R. Allen, and T. W. N. Haine, 2000: Sensitivity analysis of the climate of a chaotic system. Tellus A, 52, 523-532. 
Lehoucq, R. B., D. C. Sorensen, and C. Yang, 1998: ARPACK users' guide: solution of large-scale eigenvalue problems with implicitly restarted Arnoldi methods. Vol. 6. Siam.

Lermusiaux, P. F. J., and A. Robinson, 1999: Data assimilation via error subspace statistical estimation. Part I: Theory and schemes. Mon. Weather Rev., 127, 1385-1407.

Losch, M., and P. Heimbach, 2007: Adjoint sensitivity of an ocean general circulation model to bottom topography. J. Phys. Oceanogr., 37, 377-393.

Losch, M., and C. Wunsch, 2003: Bottom topography as a control variable in an ocean model. J. Atmos. Ocean. Technol., 20, 1685-1696.

Magnus, J. R., and H. Neudecker, 1988: Matrix differential calculus with applications in statistics and econometrics, John Wiley \& Sons.

Marotzke, J., R. Giering, K. Q. Zhang, D. Stammer, C. Hill, and T. Lee, 1999: Construction of the adjoint MIT ocean general circulation model and application to Atlantic heat transport sensitivity. Journal of geophysical Research, 104, 529-548.

Marshall, J., A. Adcroft, C. Hill, L. Perelman, and C. Heisey, 1997: A finite-volume, incompressible Navier Stokes model for studies of the ocean on parallel computers. Journal of Geophysical Research, 102, 5753-5766.

Marshall, J., C. Hill, L. Perelman, and A. Adcroft, 1997: Hydrostatic, quasi-hydrostatic, and nonhydrostatic ocean modeling. Journal of Geophysical Research, 102, 5733-5752.

Mazloff, M. R., P. Heimbach, and C. Wunsch, 2010: An eddy-permitting Southern Ocean state estimate. J. Phys. Oceanogr., 40, 880-899.

Meredith, M. P., and coauthors, 2011: Sustained monitoring of the Southern Ocean at Drake Passage: Past achievements and future priorities. Rev. Geophys., 49, RG4005.

Moore, A. M., H. G. Arango, G. Broquet, B. S. Powell, A. T. Weaver, and J. ZavalaGaray, 2011: The Regional Ocean Modeling System (ROMS) 4-dimensional variational data assimilation systems Part I-System overview and formulation. Prog. Oceanogr., 91, 34-49.

Oden, J. T., Moser, R., and Ghattas, O. 2010: Computer Predictions with Quantified Uncertainty, Part I. SIAM News, 43(9), 1-3.

Olbers, D., D. Borowski, C. Volker, and J. Wolff, 2004: The dynamical balance, transport and circulation of the Antarctic Circumpolar Current. Antarct. Sci., 16, 439-470.

Olbers, D., and K. Lettmann, 2007: Barotropic and baroclinic processes in the transport variability of the Antarctic Circumpolar Current. Ocean Dynamics, 57, 559-578.

Rintoul, S. R., C. W Hughes, and D. Olbers, 2001: The Antarctic circumpolar current system. International Geophysics, 77, 271-XXXVI.

Rogers, G. S., 1980: Matrix derivatives, Lecture Notes in Statistics, Marcel Dekker.

Seber, G. A. F., and C. J. Wild, 2003: Nonlinear regression. Vol. 503. Wiley-IEEE.

Taylor, J. R., 1997: An introduction to error analysis: the study of uncertainties in physical measurements, Univ. Science Books. 
Thacker, W. C., 1989: The role of the Hessian matrix in fitting models to measurements. Journal of Geophysical Research, 94, 6177-6196.

Trefethen, L. N., and D. Bau III, 1997: Numerical linear algebra, Society for Industrial Mathematics.

Versée, F., 1999: Variable-storage quasi-Newton operators as inverse forecast/analysis error covariance matrices in variational data assimilation. Rapports de recherche-INRIA.

Wearn Jr, R. B., and D. J. Baker Jr, 1980: Bottom pressure measurements across the Antarctic Circumpolar Current and their relation to the wind. Deep Sea Research Part A.Oceanographic Research Papers, 27, 875-888.

Weijer, W., and S. T. Gille, 2005: Adjustment of the Southern Ocean to wind forcing on synoptic time scales. J. Phys. Oceanogr., 35, 2076-2089.

Whitworth III, T., 1983: Monitoring the transport of the Antarctic circumpolar current at Drake Passage. J. Phys. Oceanogr., 13, 2045-2057.

Whitworth III, T., and R. Peterson, 1985: Volume Transport of the Antarctic Circumpolar Current from Bottom Pressure Measurements. J. Phys. Oceanogr., 15, 810-816.

Wunsch, C., 2006: Discrete inverse and state estimation problems: with geophysical fluid applications. Cambridge Univ. Pr.

Wunsch, C., and P. Heimbach, 2007: Practical global oceanic state estimation. Physica $D$, 230, 197-208.

Zanna, L., P. Heimbach, A. M. Moore, and E. Tziperman, 2010: The role of ocean dynamics in the optimal growth of tropical SST anomalies. J. Phys. Oceanogr., 40, 9831003. 